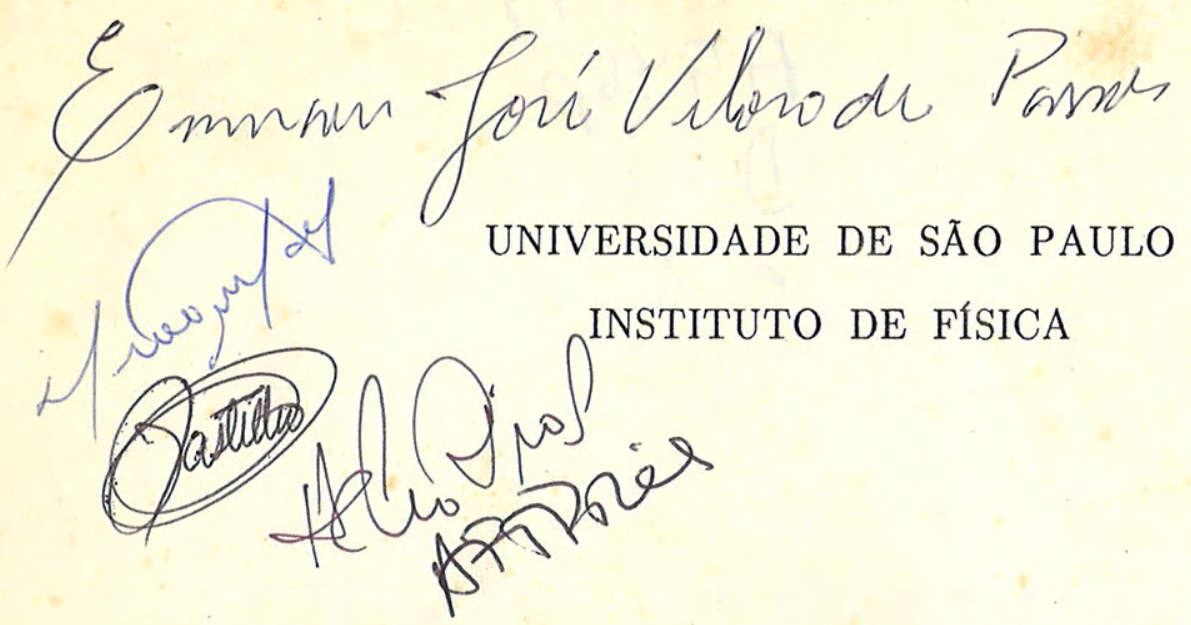

\title{
PROPRIEDADES DO MODELO SIMPLÉTICO E SEUS SUBMODELOS, QUANDO APLICADOS A NÚCLEOS LEVES
}

\section{SIDNEY DOS SANTOS AVANCINI}

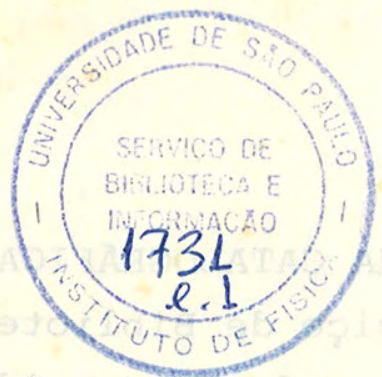

Tese de Doutoramento apresentada no Instituto de Física da Universidade de São Paulo

Orientador: Prof. Dr. Emerson José Veloso de Passos

SÃO PAULO

1990

SBI-IFUSP

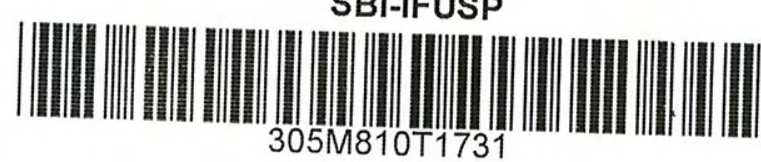




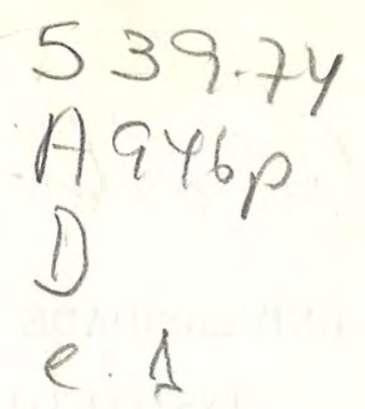

FICHA CATALOGRAFICA

Preparada pelo Serviço de Biblioteca e Informação do Instituto de Física da Universidade de São Paulo

Avancini, Sidney dos Santos

Propriedades do modelo simplético e seus submodelos, quando aplicados a núcleos leves. São Paulo, 1990.

Tese (Doutorado) - Universidade de São Paulo. Ins tituto de Física. Departamento de Física e Matemática.

Área de Concentração: Física Nuclear

Orientador: Profo Dr. Emerson José Veloso de Passos

Unitermos: 1. Estrutura nuclear; 2. Propriedades espectroscópicas de núcleos leves; 3 . Modelos coleti vos algébricos.

$\mathrm{USP} / \mathrm{IF} / \mathrm{SBI}-45 / 90$ 


\section{ÍNDICE}

Capítulo 1 - Introdução............................................................................................. 1

Capítulo 2 - Modelo Simplético ....................................................................................... 6

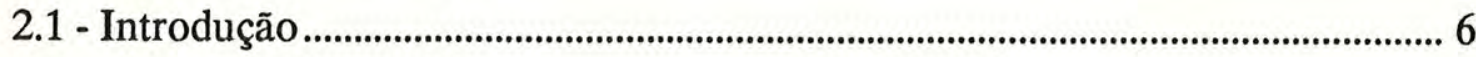

2.2 - Álgebra Simplética .............................................................................................. 7

2.3 - Construção das representações irredutíveis do grupo $\operatorname{Sp}(3, \mathrm{R})$........................ 10

2.4 - Separação da componente espúria do centro de massa .................................... 14

Capítulo 3 - Construção dos Estados de Peso Mínimo ............................................... 20

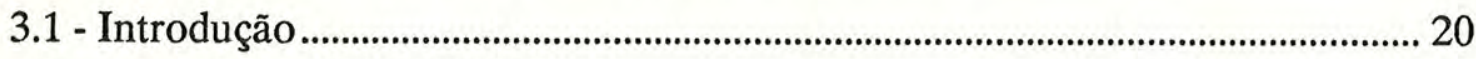

3.2 - Configurações não-excitadas........................................................................... 20

3.2.1 - Determinação da representação irredutível [444] (04) .................................. 23

3.2.2 - Determinação da representação irredutível [4431] (12) ............................... 27

3.2.3 - Determinação da representação irredutível [4422] (20) ................................ 31

3.1 - Construção dos estados de peso mínimo excitados ............................................. 36

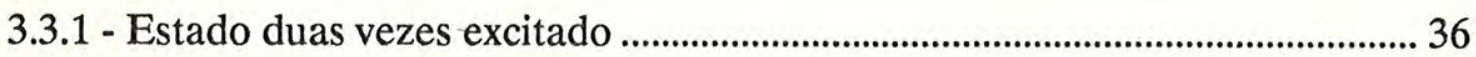

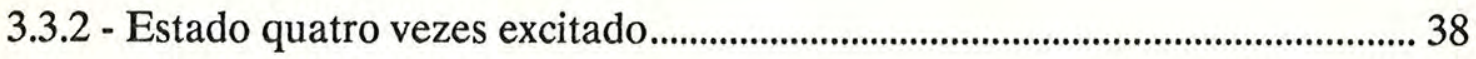

Capítulo 4 - Aplicação do Modelo Simplético à Descrição dos

Níveis de Baixa Energia do ${ }^{12}$ C.............................................................. 40

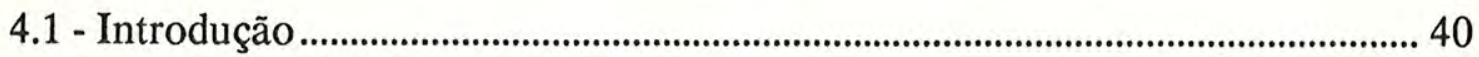

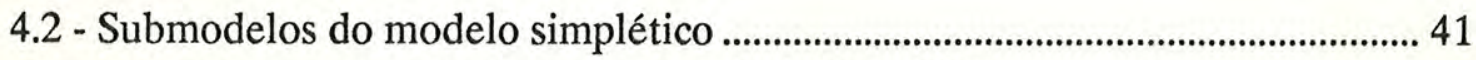

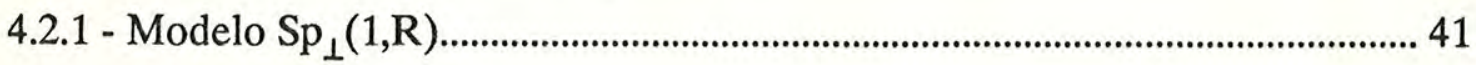

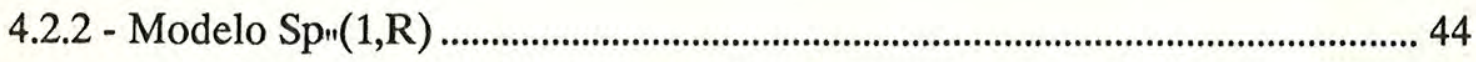

4.3 - Determinação do Subespaço Coletivo ……………………………………......... 47

4.3.1 - Modelo $\mathrm{Sp}_{\perp}(1, \mathrm{R})$ associado ao estado de peso mínimo 0p-0b...................... 48

4.3.2 - Modelo $\mathrm{Sp}_{n}(1, \mathrm{R})$ associado ao estado de peso mínimo $2 \mathrm{p}-2 \mathrm{~b}$....................... 51

4.3.3 - Modelo $\mathrm{Sp}$ "(1,R) associado ao estado de peso mínimo 4p-4b...................... 54 


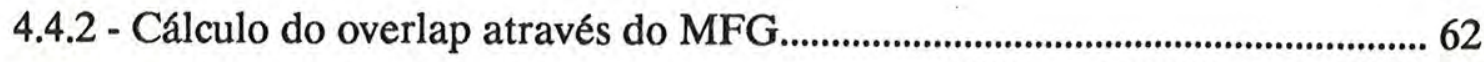

4.4.3 - Cálculo do potencial através do MFG ............................................................ 64

4.4.4 - Cálculo algébrico da energia cinética............................................................. 66

4.4.5 - Cálculo algébrico do operador momento de monopolo................................ 67

4.4.6 - Cálculo algébrico do operador momento de quadrupolo .............................. 69

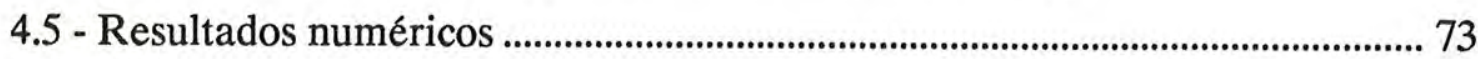

4.5.1 - Submodelo $\mathrm{Sp}_{\perp}(1, \mathrm{R})$ determinado pelo estado de peso mínimo 0p-0b .... 73

4.5.2 - Submodelo Sp"(1,R) determinado pelo estado de peso mínimo 2p-2b ..... 81

4.5.2 - Submodelo $\mathrm{Sp}_{\text {" }}(1, \mathrm{R})$ determinado pelo estado de peso mínimo $4 \mathrm{p}-4 \mathrm{~b}$..... 91

Capítulo 5 - Cálculo Sp"(1,R) em uma base deformada ............................................. 98

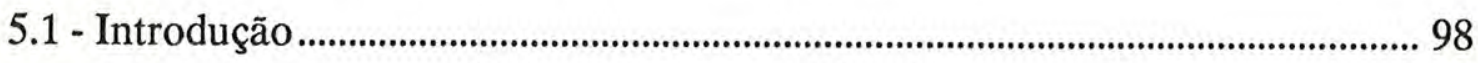

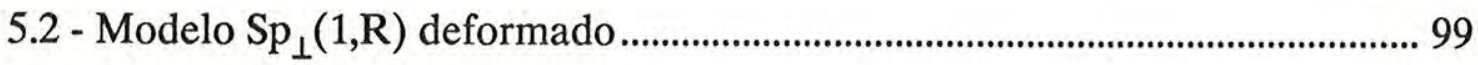

5.2.1 - Modelo $\mathrm{Sp}_{\perp}(1, \mathrm{R})$ deformado determinado

pelo estado de peso mínimo 0p-0b ............................................................. 103

5.3 - Modelo Sp"(1,R) deformado....................................................................... 111

5.3.1 - Modelo $\mathrm{Sp}_{\mathrm{N}}(1, \mathrm{R})$ deformado determinado

pelo estado de peso mínimo 2p-2b ............................................................ 116

5.3.2 - Modelo Sp" $(1, R)$ deformado determinado

pelo estado de peso mínimo 4p-4b ............................................................ 128

Capítulo 6 - Efeito da Mistura de Representações Irredutíveis de $\operatorname{Sp}(1, \mathrm{R})$...... 135

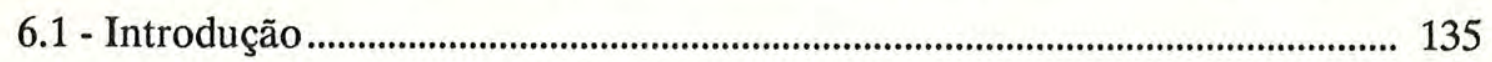

6.2 - Níveis de baixa energia do ${ }^{12} \mathrm{C}$ e a mistura de representações irredutíveis 135

6.3 - Cálculo do acoplamento entre representações irredutíveis

de Sp $(1, \mathrm{R})$ através do MFG................................................................... 137

6.3.1 - Acoplamento entre as representações 0p-0b e 4p-4b ................................ 137

6.3.2 - Acoplamento entre as representações 0p-0b e 2p-2b ................................ 141 
6.3.3 - Acoplamento entre as representações 2p-2b e 4p-4b ................................ 144

6.4 - Análise dos resultados obtidos........................................................................ 147

Capítulo 7 - Conclusão................................................................................................ 152

Apêndice 1 - Cálculo Detalhado dos Elementos de Matriz dos Operadores de Interesse........................................................... 156

A.1.1 - Cálculo através do MFG .............................................................................. 156

A.1.2 - Cálculo do overlap...................................................................................... 157

A.1.2.1 - Cálculo do overlap da representação 0p-0b ........................................... 157

A.1.2.2 - Cálculo do overlap da representação 2p-2b .............................................. 163

A.1.2.3 - Cálculo do overlap da representação 4p-4b ............................................. 169

A.1.3 - Cálculo do Potencial ........................................................................................ 170

A.1.3.1 - Cálculo do potencial através do MFG .................................................... 170

A.1.3.2 - Cálculo do elemento de matriz $\left\langle\phi_{\mathrm{i}} \phi_{\mathrm{j}}|\mathrm{u}| \tilde{\phi}_{\mathrm{k}} \tilde{\phi}_{\mathrm{l}}>\right.$................................... 172

A.1.3.3 - Cálculo do potencial na representação 0p-0b ........................................... 176

A.1.3.4 - Cálculo do potencial na representação 2p-2b ......................................... 182

A.1.3.5 - Cálculo do potencial na representação 4p-4b......................................... 188

Apêndice 2 - Forma Normal dos Operadores de Interesse................................... 189

A.2.1 - Forma Normal de Um Operador do Grupo Sp(3,R)................................ 189

A.2.2 - Representação dos Operadores de Interesse.............................................. 200

A.2.2.1 - Operador de rotação ............................................................................. 200

A.2.2.2 - Operador transformação de escala........................................................ 202

A.2.2.3 - Representação do operador $u(\rho)$........................................................ 203

A.2.3 - Cálculo da Forma Normal dos Operadores de Interesse.......................... 205

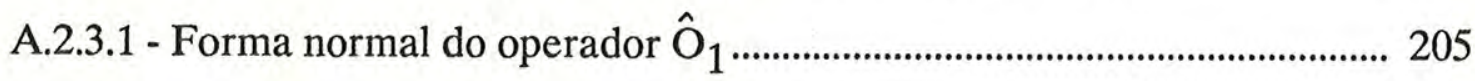

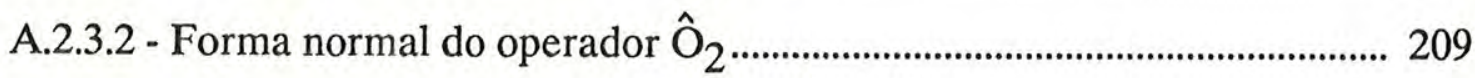

Apêndice 3 - Cálculo dos Coeficientes de Talmi-Moshinsky............................... 214 
Aos meus pais

A Tereza, minha segunda mãe

A Sandra

Ao Daniel

Aos meus irmãos 


\section{Agradecimentos}

Ao Prof. Dr. Emerson José Veloso de Passos que de maneira competente e paciente orientou este trabalho e sobretudo pelo profissionalismo que sempre transmitiu nestes anos de convivência e a quem hoje considero um amigo.

A Sandra pela compreensão e carinho, mesmo nas fases mais difíceis deste trabalho.

A Selma pelo excelente trabalho de edição de texto e paciência nas intermináveis correções de um trabalho tão longo.

Aos meus amigos que, direta ou indiretamente, me ajudaram a terminar este trabalho.

A FAPESP pelo apoio financeiro na maior parte deste trabalho e a Capes pelo apoio nos últimos seis meses. 


\begin{abstract}
In this thesis, we present a qualitative analysis of an $\mathrm{Sp}(1, \mathrm{R})$ calculation, applied to the description of the spectroscopic properties of ${ }^{12} \mathrm{C}$. Based on a calculation in a deformed harmonic oscillator basis, we show that, the need to include highly excited basis states to describe the properties of ${ }^{12} \mathrm{C}$, is due to a lack of optimization of the spherical basis. The optimized basis is given by the angular momentum projection of deformed phonon states, determined by the method of variation after projection, where the deformed phonons are associated to giant monopole and quadrupole resonances.

We have also shown that, for an adequate description of the electromagnetic transitions between states belonging to different irreducible representations of $\operatorname{Sp}(3, \mathrm{R})$, it is necessary to consider mixture of representations. In a preliminary calculation we discuss the effect of this mixture to the monopole and quadrupole transitions between the intruder state $\mathrm{O}_{2}^{+}$and the states of the ground rotational band associated, respectively, to a prolate and oblate representations.
\end{abstract}




\section{Resumo}

Analisamos de maneira qualitativa as propriedades da base de estados do modelo simplético SCM, através de um cálculo em seu submodelo $\mathrm{Sp}(1, \mathrm{R})$ que aplicamos à descrição das propriedades espectroscópicas do núcleo ${ }^{12} \mathrm{C}$.

Através de um cálculo em uma base deformada, mostramos que a necessidade de considerarmos estados de base altamente excitados para descrevermos os observáveis pelo SCM, se devia a uma falta de otimização da base. A base otimizada é dada pela projeção em momento angular de estados de fonons deformados, determinados pelo método da projeção antes da variação. Esses fonons estão relacionados com as ressonâncias gigantes monopolares e quadrupolares.

Mostramos que para descrevermos adequadamente as transições eletromagnéticas entre estados pertencentes a representações irredutíveis distintas de $\operatorname{Sp}(3, \mathrm{R})$, era necessário considerar a mistura de representações. Através de um cálculo exploratório, analisamos o efeito dessa mistura nas transições monopolares e quadrupolares entre o estado intruso $0_{2}^{+}$, que é associado a uma representação prolata de $\operatorname{Sp}(1, \mathrm{R})$ e os estados da banda rotacional fundamental, que são associados a uma representação oblata de $\operatorname{Sp}(1, \mathrm{R})$. 


\section{Introdução}

O modelo coletivo simplético ${ }^{(1)}, \mathrm{SCM}$, consiste em uma teoria microscópica que possibilita identificar as configurações do modelo de camadas do oscilador adequadas à descrição das rotações e vibrações monopolares e quadrupolares do núcleo atômico. O SCM é um modelo algébrico, onde os operadores coletivos formam a álgebra simplética $\mathrm{sp}(3, \mathrm{R})$ e são expressos em termos microscópicos. O subespaço coletivo é identificado com o espaço de uma representação irredutível do grupo $\mathrm{Sp}(3, \mathrm{R})$, que é um subespaço do espaço de Hilbert do modelo de camadas do oscilador harmônico. A hamiltoniana coletiva é identificada com a restrição de uma hamiltoniana microscópica ao subespaço coletivo. A álgebra $\mathrm{sp}(3, \mathrm{R})$ é formada pelas seis componentes do tensor de quadrupolo, seis geradores de deformações quadrupolares e monopolares, seis componentes do tensor energia cinética e três operadores de momento angular ${ }^{(1)}$. O SCM pode ser considerado como uma generalização do modelo $\mathrm{SU}(3)$ de Elliott ${ }^{(2)}$. Neste modelo todos os estados tem a mesma energia de oscilador. No SCM além dos estados de SU(3), temos estados com energia de excitação $2 \mathrm{n} \hbar \omega$ em relação aos estados de $\mathrm{SU}(3)$.

A aplicação do modelo SCM, apresenta grandes dificuldades, tanto do ponto de vista algébrico quanto computacional e por isto apenas cálculos esquemáticos usando o SCM foram feitos até o momento. Surge então a necessidade de considerarmos submodelos do SCM, onde suprimimos alguns dos seus graus de liberdade com a finalidade de ganharmos viabilidade computacional. Convém salientar que o estudo de submodelos também se justifica do ponto de vista de procurarmos separar os graus de liberdade relevantes do problema.

Um dos submodelos de uso mais frequente é o modelo $\operatorname{Sp}(1, \mathrm{R})^{(3,4)}$. Neste modelo o subespaço coletivo é obtido pela projeção em momento angular dos estados de base de uma representação irredutível do grupo $\mathrm{Sp}(1, \mathrm{R})$. Em geral para núcleos prolatos esta representação é obtida acrescentando-se sucessivamente dois quanta de oscilador na 
direção do eixo de simetria axial, o que denominaremos de modelo $\mathrm{Sp}_{\|}(1, \mathrm{R})$. Para núcleos oblatos acrescentamos quanta no plano perpendicular ao eixo de simetria axial e temos neste caso o que denominaremos modelo $\mathrm{Sp}_{\perp}(1, \mathrm{R})$. Na literatura existem vários cálculos relacionados com a aplicação do modelo $\mathrm{Sp}(1, \mathrm{R})$ a núcleos leves ${ }^{(3-8)}$. Analisando os resultados desses cálculos, notamos que para descrevermos adequadamente propriedades espectroscópicas através desse modelo é necessário considerar estados de base com alta energia de excitação.

Nesses cálculos, frequentemente são encontradas bases onde o número de camadas do oscilador, que contribuem de maneira significativa, são da ordem de 20. Assim é evidente a importância das configurações excitadas. Isto obscurece a interpretação física dos mecanismos reponsáveis pelo aparecimento das configurações excitadas, bem como impõe problemas de convergência nos cálculos. Além disto, é inviável a análise sistemática de cálculos que envolvam a mistura de representações irredutíveis de $\mathrm{Sp}(3, \mathrm{R})$.

A chave para a elucidação desse problema está na observação de que em núcleos leves as correlações oriundas da deformação são importantes. Por isso quando usamos estados de partícula independente do oscilador harmônico esférico, para descrevermos os efeitos das correlações da deformação precisamos introduzir configurações altamente excitadas. Isto sugere que uma base otimizada deve ser construída em termos de estados de partícula independente de um oscilador harmônico deformado ao invés de um oscilador harmônico esférico.

Observando que uma mudança de escala nas direções espaciais é uma transformação unitária simplética, pode-se construir uma base de estados em termos de estados de partícula independente de um oscilador harmônico deformado, que é equivalente à base de estados em termos de estados de partícula independente de um oscilador harmônico esférico.

No modelo $\mathrm{SU}(3)$ de Elliott, os estados que vão gerar o subespaço coletivo, são obtidos pela projeção em momento angular do estado de peso $\left[\begin{array}{l}\text { mínimo } \\ \text { máximo }\end{array}\right]$ de $\mathrm{SU}(3)$. Estes 
estados dependem do comprimento do oscilador harmônico esférico $b_{0}$, que é fixado variacionalmente. Em uma base deformada, o estado de peso $\left[\begin{array}{l}\text { mínimo } \\ \text { máximo }\end{array}\right]$ de $\mathrm{SU}(3)$ deformado, vai depender dos comprimentos do oscilador associados às três direções espaciais. Fixamos tais parâmetros variacionalmente, onde primeiro projetamos em momento angular o estado de peso $\left[\begin{array}{l}\text { mínimo } \\ \text { máximo }\end{array}\right]$. Este procedimento é chamado de método da projeção antes da variação $\mathrm{PAV}^{(10)}$.

O nosso objetivo no trabalho de tese é realizar uma análise qualitativa dos resultados de um cálculo do SCM em uma base otimizada. Os resultados dessa análise no ${ }^{8} \mathrm{Be}$, para a representação irredutível (40), foram discutidos na ref.(9). Para essa representação irredutível os estados otimizados são prolatos e uma análise dos resultados mostra que os estados da banda fundamental são essencialmente dados pela projeção em momento angular de um único estado, interpretado como o vácuo de um fonon deformado. A inclusão de estados com um e dois fonons praticamente reproduz os resultados da conta exata, no contexto do modelo. Esse resultado mostra que o modelo $\mathrm{Sp}(1, \mathrm{R})$ pode ser visto como uma generalização do método projeção antes da variação do oscilador harmônico deformado de Abgral et al ${ }^{(10)}$ e Bouten \& Bouten ${ }^{(11)}$.

Assim esse trabalho mostra a factibilidade de um cálculo numérico em uma base deformada e resolve os problemas de convergência e interpretação física de um cálculo na base esférica. No trabalho de tese extendemos os cálculos da ref.(9) para incluir representações irredutíveis de $\mathrm{Sp}(1, \mathrm{R})$ construídas com estados oblatos e triaxiais, com o objetivo de verificar se as conclusões da ref.(9) continuam válidas nesse caso. Os três tipos de representações irredutíveis de $\mathrm{Sp}(1, \mathrm{R})$, citadas acima, surgem naturalmente quando procuramos descrever o espectro de baixa energia do ${ }^{12} \mathrm{C}$.

$\mathrm{O}$ cálculo do tipo $\mathrm{Sp}_{\perp}(1, \mathrm{R})$ feito por Arickx et al ${ }^{(4)}$, nos mostra que a banda rotacional fundamental do ${ }^{12} \mathrm{C}$ é associada por este modelo à uma representação irredutível de $\mathrm{Sp}(1, \mathrm{R})$ oblata, com rótulos (04) de $\mathrm{SU}(3)$. No espectro de baixa energia 
do ${ }^{12} \mathrm{C}$, é observado um estado $0_{2}^{+}$a uma energia de excitação de $7.65 \mathrm{MeV}$, que é interpretada por Bouten et al ${ }^{(15)}$ como sendo um estado intruso, associado ao estado de peso mínimo de $\mathrm{SU}(3)$ prolato com rótulos $(120)$ de $\mathrm{SU}(3)$. A um possível estado $0_{3}^{+}$, com energia de excitação $10.3 \mathrm{MeV}$, Bouten et al ${ }^{(15)}$ procura associar um estado de peso mínimo de $\mathrm{SU}(3)$ triaxial com rótulos (62) de $\mathrm{SU}(3)$. Portanto, consideramos em nosso estudo da base otimizada cálculos do tipo $\mathrm{Sp}_{\|}(1, \mathrm{R})$ a partir dos estados de peso mínimo de $\mathrm{SU}(3),(120)$ e $(62)$ e o cálculo $\operatorname{Sp}_{\perp}(1, \mathrm{R})$ a partir do estado $(04)$.

Finalmente, mostramos a viabilidade de realizarmos um cálculo onde levamos em conta a mistura de representações irredutíveis de $\operatorname{Sp}(3, \mathrm{R})$. A necessidade de considerarmos tal mistura, surge naturalmente quando analisamos as transições eletromagnéticas entre os estados gerados pelo modelo, que procura descrever o espectro de baixa energia através dos três modelos $\mathrm{Sp}(1, \mathrm{R})$ que citamos acima. Como os operadores de monopolo e quadrupolo pertencem à álgebra $\mathrm{sp}(3, \mathrm{R})$, transições monopolares e quadrupolares entre representações irredutíveis diferentes de $\operatorname{Sp}(3, \mathrm{R})$ são proibidas pelo modelo. Por exemplo, experimentalmente, observa-se transições monopolares entre os estados $0_{2}^{+}$e $0_{1}^{+}$e quadrupolares entre os estados $0_{2}^{+}$e $2_{1}^{+}$. Como vimos acima tais estados pertencem a representações irredutíveis diferentes de $\mathrm{Sp}(1, \mathrm{R})$ e portanto são proibidas.

Para procurar descrever tais transições, realizamos um cálculo exploratório onde levamos em conta a mistura de representações irredutíveis. O procedimento mais simples seria considerar o acoplamento entre as representações oblatas e prolatas, mas no entanto, mostra-se que não existe interação de dois corpos que produza tal acoplamento.

Portanto, precisamos levar em conta uma terceira representação irredutível para, de maneira indireta, acoplarmos as representações oblatas e prolatas e sendo assim, somos levados a considerar a representação triaxial com esta finalidade.

Organizamos este trabalho da seguinte maneira: 
No capítulo 2 fazemos uma análise do modelo simplético e mostramos como em princípio podemos construir uma base de estados do mesmo. No capítulo 3 mostramos como são construídos os estados de peso mínimo de $\mathrm{Sp}(3, \mathrm{R})$, que são adequados à descrição do espectro de baixa energia do ${ }^{12} \mathrm{C}$. No capítulo 4 discutimos o método que utilizamos no cálculo dos elementos de matriz da hamiltoniana e dos operadores de transição eletromagnéticas na base de estados do modelo $\mathrm{Sp}(1, \mathrm{R})$, obtida a partir de funções de onda do oscilador harmônico esférico. Analisamos a aplicação deste modelo ao ${ }^{12} \mathrm{C}$, onde consideramos estados de peso mínimo oblato, prolato e triaxial e discutimos os resultados numéricos obtidos. No capítulo 5 consideramos o cálculo do modelo $\mathrm{Sp}(1, \mathrm{R})$ em uma base deformada equivalente à base esférica. Analisamos a aplicação deste modelo aos estados de baixa energia do ${ }^{12} \mathrm{C}$, de maneira paralela ao que fizemos no capítulo $4 \mathrm{e}$ estudamos a relação do modelo $\mathrm{Sp}(1, \mathrm{R})$ deformado com o método PAV. No capítulo 6 realizamos um cálculo envolvendo a mistura das representações irredutíveis de $\operatorname{Sp}(1, \mathrm{R})$, obtidas a partir dos estados de peso mínimo oblato, prolato e triaxial. Analisamos o efeito de tal mistura no cálculo das transições monopolares e quadrupolares entre os estados $0_{2}^{+} \longrightarrow 0_{1}^{+}$e $0_{2}^{+} \rightarrow 2_{1}^{+}$, respectivamente.

Dada a complexidade dos cálculos no contexto do modelo coletivo simplético, tivemos que desenvolver várias técnicas algébricas e numéricas, para o cálculo dos elementos de matriz dos observáveis de interesse. Uma exposição sumária dessas técnicas é apresentada nos apêndices. 


\section{Modelo Simplético}

\subsection{Introdução}

O modelo simplético ${ }^{(1)}$, SCM, consiste em uma teoria microscópica coletiva algébrica, que possibilita identificar as configurações do modelo de camadas do oscilador adequadas à descrição das vibrações monopolares e quadrupolares do núcleo atômico. Do ponto de vista fenomenológico, identificamos as vibrações monopolares e quadrupolares respectivamente, com oscilações compressionais e elipsoidais isovolumétricas da forma de equilíbrio do núcleo.

Uma vez que os geradores dessas oscilações não formam uma álgebra de Lie é necessário incluirmos os operadores de momento angular, obtendo assim, a álgebra denominada $g l(3, R)$. A fim de construirmos um subespaço coletivo que descreva as excitações monopolares e quadrupolares acrescentamos os operadores monopolo e quadrupolo aos geradores de $\mathrm{gl}(3, \mathrm{R})$ obtendo a álgebra soma semidireta de Lie, $\mathrm{cm}(3)$.

Para construir um modelo que possa a priori ser imerso no modelo de camadas do oscilador adicionamos o tensor energia cinética à álgebra $\mathrm{cm}(3)$, obtendo deste modo a álgebra simplética $\mathrm{sp}(3, \mathrm{R})$, que contém a hamiltoniana do oscilador harmônico 3-dimensional.

No SCM, o subespaço coletivo é identificado com o espaço de uma representação irredutível do grupo simplético associado à álgebra dos operadores coletivos. A álgebra simplética, $\mathrm{sp}(3, \mathrm{R})$, é realizada em termos microscópicos por meio de operadores de um corpo, e o espaço de uma representação irredutível do grupo simplético é um subespaço do espaço de Hilbert do modelo de camadas do oscilador. A hamiltoniana coletiva é identificada com a restrição da hamiltoniana nuclear de muitos corpos ao subespaço coletivo. 
Vamos a seguir descrever de maneira concisa como podemos em princípio, construir os estados de base do SCM, objetivando fixar a notação futura e mostrar o contexto mais geral em que estarão contidos os submodelos do SCM que utilizaremos nos capítulos posteriores.

\section{2. Álgebra Simplética}

Os operadores responsáveis pela deformação da forma de equilíbrio do núcleo são dados por:

$$
\mathrm{S}_{\mu \nu}=\frac{1}{2} \sum_{\mathrm{k}=1}^{\mathrm{A}}\left[\mathrm{x}_{\mu}(\mathrm{k}) \mathrm{p}_{\nu}(\mathrm{k})+\mathrm{p}_{\mu}(\mathrm{k}) \mathrm{x}_{\nu}(\mathrm{k})\right]
$$

onde $\mu, \nu=1,2,3$ corresponde às direções espaciais e o rótulo $\mathrm{k}$ está associado ao k-ésimo nucleon. Os elementos diagonais $\mathrm{S}_{\mu \mu}$, correspondem aos geradores de transformação de escala na direção $\mu$.

Através do cálculo direto é fácil ver que os operadores acima não se fecham por comutação e portanto não formam uma álgebra de Lie. Com a inclusão dos operadores de momento angular dados por:

$$
\mathrm{L}_{\mu \nu}=\frac{1}{2} \sum_{\mathrm{k}=1}^{\mathrm{A}}\left[\mathrm{x}_{\mu}(\mathrm{k}) \mathrm{p}_{\nu}(\mathrm{k})-\mathrm{x}_{\nu}(\mathrm{k}) \mathrm{p}_{\mu}(\mathrm{k})\right]
$$

obtemos o menor conjunto de operadores que contém $\mathrm{S}_{\mu \nu}$ e se fecha por comutação. Este conjunto de nove operadores, geram a álgebra de Lie denominada $\mathrm{gl}(3, \mathrm{R})$. 
A fim de descrevermos as excitações monopolares e quadrupolares adicionamos à álgebra $\mathrm{gl}(3, \mathrm{R})$ os operadores de monopolo e quadrupolo, dados por:

$$
\mathrm{Q}_{\mu \nu}=\sum_{\mathrm{k}=1}^{\mathrm{A}} \mathrm{x}_{\mu}(\mathrm{k}) \mathrm{x}_{\nu}(\mathrm{k})
$$

O conjunto de 15 operadores, $\mathrm{S}_{\mu \nu}, \mathrm{L}_{\mu \nu}, \mathrm{Q}_{\mu \nu}$ geram a álgebra $\mathrm{cm}(3)$. Operadores $\mathrm{Q}_{\mu \nu}$ comutam entre si e portanto formam a álgebra de Lie denominada $R^{6}$. Assim $\mathrm{cm}(3)$ tem $\operatorname{gl}(3, R)$ e $\mathbf{R}^{6}$ como subálgebras. Como a comutação entre $\mathbf{R}^{6}$ e $\operatorname{gl}(3, \mathrm{R})$ resulta em elementos pertencentes a $\mathrm{R}^{6}$, isto é:

$$
\left[\mathrm{R}^{6}, \operatorname{gl}(3, \mathrm{R})\right] \subset \mathrm{R}^{6}
$$

dizemos que $\mathrm{cm}(3)$ é uma álgebra soma semidireta de Lie, que é representada pela seguinte notação:

$$
\mathrm{cm}(3)=\mathrm{R}^{6} \ddot{\oplus} \mathrm{gl}(3, \mathrm{R})
$$

Com o acréscimo do tensor energia cinética,

$$
\mathrm{K}_{\mu \nu}=\sum_{\mathrm{k}=1}^{\mathrm{A}} \mathrm{p}_{\mu}(\mathrm{k}) \mathrm{p}_{\nu}(\mathrm{k})
$$

à álgebra $\mathrm{cm}(3)$ obtemos o conjunto de 21 operadores, $\mathrm{S}_{\mu \nu}, \mathrm{L}_{\mu \nu}, \mathrm{Q}_{\mu \nu}, \mathrm{K}_{\mu \nu}$, que se fecham por comutação e geram a álgebra de Lie simplética, $\mathrm{sp}(3, \mathrm{R})$. A fim de 
construirmos as representações irredutíveis de $\mathrm{Sp}(3, \mathrm{R})$, vamos obter uma realização equivalente da álgebra $\mathrm{sp}(3, \mathrm{R})$ no espaço de Fock ${ }^{(1)}$.

Definindo os operadores criação e aniquilação de quanta de oscilador por:

$$
\begin{aligned}
& \mathrm{a}_{\mu ; \mathrm{k}}^{+\mathrm{e}}\left(\mathrm{b}_{0}\right)=\frac{1}{\sqrt{2}}\left[\frac{\mathrm{x}_{\mu}(\mathrm{k})}{\mathrm{b}_{0}}-\mathrm{i} \frac{\mathrm{b}_{0}}{\hbar} \mathrm{p}_{\mu}(\mathrm{k})\right] \\
& \mathrm{a}_{\mu ; \mathrm{k}}^{\mathrm{e}}\left(\mathrm{b}_{0}\right)=\left[\mathrm{a}_{\mu ; \mathrm{k}}^{+\mathrm{e}}\left(\mathrm{b}_{0}\right)\right]^{+}
\end{aligned}
$$

onde $b_{0}=\sqrt{\frac{\hbar}{m \omega}}$ corresponde ao comprimento do oscilador. O indice superior "e" indica que os operadores estão associados a um referencial fixo no espaço.

Por meio do produto bilinear dos operadores acima obtemos a seguinte realização da álgebra $\mathrm{sp}(3, \mathrm{R})$ no espaço de Fock:

$$
\begin{aligned}
& \mathrm{A}_{\mu \nu}^{+\mathrm{e}}\left(\mathrm{b}_{0}\right)=\frac{1}{2} \sum_{\mathrm{k}=1}^{\mathrm{A}} \mathrm{a}_{\mu ; \mathrm{k}}^{+\mathrm{e}}\left(\mathrm{b}_{0}\right) \mathrm{a}_{\nu ; \mathrm{k}}^{+\mathrm{e}}\left(\mathrm{b}_{0}\right) \\
& \mathrm{A}_{\mu \nu}^{\mathrm{e}}\left(\mathrm{b}_{0}\right)=\frac{1}{2} \sum_{\mathrm{k}=1}^{\mathrm{A}} \mathrm{a}_{\mu ; \mathrm{k}}^{\mathrm{e}}\left(\mathrm{b}_{0}\right) \mathrm{a}_{\nu ; \mathrm{k}}^{\mathrm{e}}\left(\mathrm{b}_{0}\right) \\
& \mathrm{C}_{\mu \nu}^{\mathrm{e}}\left(\mathrm{b}_{0}\right)=\frac{1}{2} \sum_{\mathrm{k}=1}^{\mathrm{A}} \mathrm{a}_{\mu ; \mathrm{k}}^{+\mathrm{e}}\left(\mathrm{b}_{0}\right) \mathrm{a}_{\nu ; \mathrm{k}}^{\mathrm{e}}\left(\mathrm{b}_{0}\right)+\mathrm{a}_{\nu ; \mathrm{k}}^{\mathrm{e}}\left(\mathrm{b}_{0}\right) \mathrm{a}_{\mu ; \mathrm{k}}^{+\mathrm{e}}\left(\mathrm{b}_{0}\right) .
\end{aligned}
$$

Para simplificar a notação, omitiremos o comprimento do oscilador, $b_{0}$, dos operadores acima nos casos em que isto não causar confusão. A partir da definição de $a_{\mu ; \mathrm{k}}^{+\mathrm{e}}$ e $\mathrm{a}_{\mu ; \mathrm{k}}^{\mathrm{e}}$ 
dada em (5) é fácil obter as seguintes expressões relacionando as duas realizações da álgebra $\mathrm{sp}(3, \mathrm{R})$ obtidas acima:

$$
\begin{aligned}
& \mathrm{Q}_{\mu \nu}=\mathrm{b}_{0}^{2}\left[\mathrm{~A}_{\mu \nu}^{+\mathrm{e}}+\mathrm{A}_{\mu \nu}^{\mathrm{e}}+\frac{1}{2}\left[\mathrm{C}_{\mu \nu}^{\mathrm{e}}+\mathrm{C}_{\nu \mu}^{\mathrm{e}}\right]\right] \\
& \mathrm{K}_{\mu \nu}=-\frac{\hbar^{2}}{\mathrm{~b}_{0}^{2}}\left[\mathrm{~A}_{\mu \nu}^{+\mathrm{e}}+\mathrm{A}_{\mu \nu}^{\mathrm{e}}-\frac{1}{2}\left[\mathrm{C}_{\mu \nu}^{\mathrm{e}}+\mathrm{C}_{\nu \mu}^{\mathrm{e}}\right]\right] \\
& \mathrm{S}_{\mu \nu}=\mathrm{i}\left[\mathrm{A}_{\mu \nu}^{+\mathrm{e}}-\mathrm{A}_{\mu \nu}^{\mathrm{e}}\right] \\
& \mathrm{L}_{\mu \nu}=\frac{\mathrm{i}}{2}\left[\mathrm{C}_{\nu \mu}^{\mathrm{e}}-\mathrm{C}_{\mu \nu}^{\mathrm{e}}\right] .
\end{aligned}
$$

\subsection{Contrução das Representações Irredutiveis do Grupo $\mathrm{Sp}(3, \mathrm{R})$}

Para construirmos as representações irredutíveis de $\mathrm{Sp}(3, \mathrm{R})$, primeiro determinamos a subálgebra de Cartan de $\operatorname{sp}(3, \mathrm{R})$ ou seja o maior subconjunto de operadores da álgebra que comutam entre si. Neste caso é fácil ver que a subálgebra de Cartan de $\mathrm{sp}(3, \mathrm{R})$ é gerada pelos operadores $\mathrm{C}_{11}^{\mathrm{e}}, \mathrm{C}_{22}^{\mathrm{e}}, \mathrm{C}_{33}^{\mathrm{e}}$.

Os operadores que formam a subálgebra de Cartan também são denominados de operadores de peso da álgebra. Os operadores levantadores de $\operatorname{sp}(3, \mathrm{R})$ são definidos como sendo $\mathrm{C}_{\mu \nu}^{\mathrm{e}}, \mu>\nu$ e $\mathrm{A}_{\mu \nu}^{+\mathrm{e}}$ para todo $\mu$ e $\nu$ e os operadores abaixadores de $\operatorname{sp}(3, \mathrm{R})$ como sendo $\mathrm{C}_{\mu \nu}^{\mathrm{e}}, \mu<\nu$ e $\mathrm{A}_{\mu \nu}^{\mathrm{e}}$ para todo $\mu$ e $\nu$.

$\mathrm{O}$ estado de peso mínimo do grupo $\mathrm{Sp}(3, \mathrm{R})$, $\left|[\mathrm{f}] \mathrm{LW}>^{\mathrm{e}}=\right|\left[\mathrm{f}_{1} \mathrm{f}_{2} \mathrm{f}_{3}\right] \mathrm{LW}>^{\mathrm{e}}$ é definido como sendo o estado que é autoestado da subálgebra de Cartan e que é aniquilado 
pelos operadores abaixadores da álgebra:

$$
\begin{aligned}
& C_{\mu \mu}^{\mathrm{e}}\left|\left[\mathrm{f}_{1} \mathrm{f}_{2} \mathrm{f}_{3}\right] L W>^{\mathrm{e}}=\left(\mathrm{f}_{\mu}+\mathrm{A} / 2\right)\right|\left[\mathrm{f}_{1} \mathrm{f}_{2} \mathrm{f}_{3}\right] L W>^{\mathrm{e}} \\
& \mathrm{A}_{\mu \mu}^{\mathrm{e}} \mid\left[\mathrm{f}_{1} \mathrm{f}_{2} \mathrm{f}_{3}\right] L W>^{\mathrm{e}}=0, \forall \mu, \nu \\
& \mathrm{C}_{\mu \nu}^{\mathrm{e}} \mid\left[\mathrm{f}_{1} \mathrm{f}_{2} \mathrm{f}_{3}\right] L W>^{\mathrm{e}}=0, \mu<\nu
\end{aligned}
$$

onde os $\mathrm{f}_{\mu}$ correspondem ao número de quanta na direção " $\mu$ ".

Como os operadores $\mathrm{C}_{\mu \nu}^{\mathrm{e}}$ geram a subálgebra $\mathrm{u}(3)$ de $\mathrm{sp}(3, \mathrm{R})$, a subálgebra de Cartan de $u(3)$ e $\operatorname{sp}(3, R)$ coincidem, e os operadores levantadores (abaixadores) de $u(3)$ são definidos por $\mathrm{C}_{\mu \nu}^{\mathrm{e}}, \mu>\nu\left(\mathrm{C}_{\mu \nu}^{\mathrm{e}}, \mu<\nu\right)$ é imediato que $\mid[\mathrm{f}] \mathrm{LW}>$ também é estado de peso mínimo de $\mathrm{U}(3)$. Do mesmo modo, segue que $\mid[\mathrm{f}] \mathrm{LW}>$ também é estado de peso mínimo de $\mathrm{SU}(3)$. Recordemos que os operadores levantadores e abaixadores das álgebras su(3) e u(3) coincidem e a subálgebra de Cartan de $\mathrm{su}(3)$ é gerada pelos operadores $\mathrm{C}_{11}^{\mathrm{e}}-\mathrm{C}_{22}^{\mathrm{e}}, \mathrm{C}_{22}^{\mathrm{e}}-\mathrm{C}_{33}^{\mathrm{e}}$.

Os estados da representação irredutível de $\mathrm{Sp}(3, \mathrm{R})$, associada ao estado de peso mínimo $\mid[f] L W>^{e}$, são obtidos pela ação repetida dos operadores levantadores da álgebra no estado $\mid[\mathrm{f}] \mathrm{LW}>^{\mathrm{e}}$, que representamos por:

$$
\mathrm{P}\left[\ldots\left\{\mathrm{A}_{\mu \nu}^{+\mathrm{e}}\right\} \ldots\right] \mathrm{P}\left[\ldots\left\{\mathrm{C}_{\alpha \beta}^{\mathrm{e}}\right\} \cdots\right] \mid[\mathrm{f}] \mathrm{LW}>\mathrm{e}
$$

onde $\mu, \nu=1,2,3, \quad \alpha>\beta$ e $\mathrm{P}(\ldots\{\} \ldots)$ indica um polinômio arbitrário nos seus argumentos.

Fica claro, na expressão acima, que o SCM é uma generalização do modelo SU(3) 
de Elliott ${ }^{(2)}$. No modelo de Elliott os estados de base tem a mesma energia de oscilador harmônico. No SCM além destes, os estados de base contém estados com energia de excitação $2 n \hbar \omega, \quad n \neq 0$, em relação aos estados do modelo de Elliott. Estes estados excitados são obtidos pela adição sucessiva de dois quanta de oscilador, pela ação dos operadores $\mathrm{A}_{\mu \nu}^{+\mathrm{e}}$ nos estados do modelo de Elliott.

Para construirmos explicitamente os estados de uma representação irredutível de $\mathrm{Sp}(3, \mathrm{R})$, primeiro notamos que os estados:

$$
\mathrm{P}\left[\ldots\left\{\mathrm{C}_{\alpha \beta}^{\mathrm{e}}\right\} \ldots\right] \mid[\mathrm{f}] \mathrm{LW}>^{\mathrm{e}}, \quad \alpha>\beta
$$

vão gerar uma representação irredutível do grupo U(3).

Notando que os operadores $\mathrm{A}_{\mu \nu}^{+\mathrm{e}}$ são as conıponentes de um tensor irredutível [200] de $\mathrm{U}(3)$ e como pode ser demonstrado, o k-ésimo produto de Kronecker desses tensores se decompõe em uma soma de tensores irredutíveis de $\mathrm{U}(3)$, dada pela seguinte regra:

$\mathrm{Z}^{[\mathrm{n}]}\left(\mathrm{A}^{+\mathrm{e}}\right)=\mathrm{N}_{\mathrm{n}}\left(\mathrm{A}^{+\mathrm{e}} \mathrm{x} \ldots \mathrm{A}^{+\mathrm{e}}\right)^{[\mathrm{n}]} \quad$ com $\quad[\mathrm{n}] \in \mathrm{D}$ onde $\mathrm{D}$ é o conjunto das partições $[\mathrm{n}]=\left\{\mathrm{n}_{1}, \mathrm{n}_{2}, \mathrm{n}_{3}\right\}$, do inteiro, $\mathrm{n}$, satisfazendo as seguintes relações:

$$
\begin{aligned}
& \mathrm{n}_{1} \geq \mathrm{n}_{2} \geq \mathrm{n}_{3} \\
& \mathrm{n}_{1}+\mathrm{n}_{2}+\mathrm{n}_{3}=2 \mathrm{k}
\end{aligned}
$$

onde $\mathrm{n}_{1}, \mathrm{n}_{2}, \mathrm{n}_{3}$ são inteiros pares não negativos e $\mathrm{N}_{\mathrm{n}}$ é uma constante de normalização. É sabido que a decomposição acima não tem multiplicidade.

Portanto, podemos obter os estados (9), que vão gerar a representação irredutível de $\mathrm{Sp}(3, \mathrm{R})$ determinada pelo estado de peso mínimo $\mid[\mathrm{f}] \mathrm{LW}>^{\mathrm{e}}$, pela seguinte expressão: 


$$
\mid[\mathrm{f}][\mathrm{n}] \rho[\omega] \alpha>^{\mathrm{e}}=\left[\mathrm{Z}^{[\mathrm{n}]}\left(\mathrm{A}^{+\mathrm{e}}\right) \mid[\mathrm{f}] \mathrm{LW}>^{\mathrm{e}}\right]_{\alpha}^{\rho \omega}
$$

onde $[\omega]=\left[\begin{array}{lll}\omega_{1} & \omega_{2} & \omega_{3}\end{array}\right]$ rotula as representações irredutíveis de $\mathrm{U}(3)$ contidas na decomposição do moduto de Kronecker $\left[\mathrm{n}_{1} \mathrm{n}_{2} \mathrm{n}_{3}\right] \times\left[\mathrm{f}_{1} \mathrm{f}_{2} \mathrm{f}_{3}\right], \rho$ é o rótulo associado à multiplicidade dessa decomposição. O rótulo $\alpha=$ KLM é associado à base de estados da representação $[\omega]$ de $U(3)$ que se transformam de acordo com a cadeia de subgrupos

$$
\begin{array}{lcccc}
\mathrm{U}(3) & \mathrm{J} & \mathrm{SO}(3) & \supset & \mathrm{SO}(2) \\
{[\omega]} & \mathrm{K} & \mathrm{L} & \mathrm{M}
\end{array}
$$

onde $\mathrm{K}$ é o rótulo associado à multiplicidade na decomposição $\mathrm{U}(3) \supset \mathrm{SO}(3)$.

Os estados (10) se transformam segundo a seguinte cadeia de subgrupos:

$$
\begin{array}{lllllll}
\mathrm{Sp}(3, \mathrm{R}) & \supset & \mathrm{U}(3) & \mathrm{U}(3) & \mathrm{SO}(3) & \supset & \mathrm{SO}(2) \\
{[\mathrm{n}] \rho} & {[\omega]} & \mathrm{K} & \mathrm{L} & & \mathrm{M}
\end{array} .
$$

Frequentemente é conveniente rotular os estados de $\mathrm{U}(3)$ pela seguinte cadeia de subgrupos:

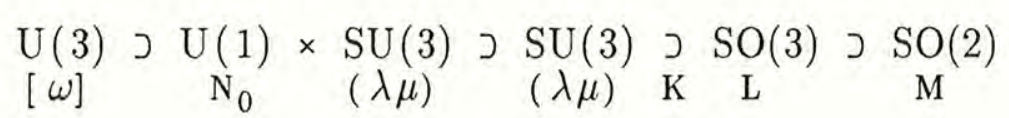

onde $\mathrm{N}_{0}=\omega_{1}+\omega_{2}+\omega_{3}$ caracteriza o subgrupo $\mathrm{U}(1)$ de $\quad \mathrm{U}(3)$ e $\quad(\lambda \mu)$ com $\lambda=\omega_{1}-\omega_{2}, \quad \mu=\omega_{2}-\omega_{3} \quad$ correspondem aos rótulos de Elliott que caracterizam a representação irredutível de $\mathrm{SU}(3)$ associada à representação $[\omega]$ de $\mathrm{U}(3)$, onde lembramos que a redução de $\mathrm{U}(3)$ ao seu subgrupo $\mathrm{SU}(3)$ não tem multiplicidade. $\mathrm{O}$ rótulo $\mathrm{K}$ está associado à multiplicidade na redução de $\mathrm{SU}(3)$ ao seu subgrupo $\mathrm{SO}(3)$. 
Os estados (10) são autoestados da hamiltoniana do oscilador harmônico tri-dimensional, $\mathrm{H}_{0}=\hbar \omega \Sigma \mathrm{C}_{\mu \mu}^{\mathrm{e}}$

$$
\mathrm{H}_{0}\left|[\mathrm{f}][\mathrm{n}] \rho[\omega] \alpha>^{\mathrm{e}}=\left(\mathrm{N}_{0}+\frac{3 \mathrm{~A}}{2}+2\left(\mathrm{n}_{1}+\mathrm{n}_{2}+\mathrm{n}_{3}\right)\right] \hbar \omega\right|[\mathrm{f}][\mathrm{n}] \rho[\omega] \alpha>^{\mathrm{e}}
$$

onde $\mathrm{N}_{0}=\mathrm{f}_{1}+\mathrm{f}_{2}+\mathrm{f}_{3}$.

\subsection{Separação da Componente Espúria do Centro de Massa}

Os operadores que formam a álgebra simplética, como mostramos na seção 2.2 , são definidos em relação a um referencial fixo no espaço e portanto vão gerar excitações coletivas que podem ser decompostas em intrínsecas e do centro de massa. Vamos a seguir mostrar como podemos eliminar as excitações do centro de massa, que correspodem a excitações espúrias, sem conteúdo físico.

Definindo a seguinte transformação ortogonal, O, dos operadores posição e momento do i-ésimo nucleon ${ }^{(13)}$ :

$$
\begin{aligned}
& \rho_{\mu}(\mathrm{i})=\sum_{\mathrm{k}=1}^{\mathrm{A}} \mathrm{O}_{\mathrm{ik}} \mathrm{x}_{\mu}(\mathrm{k}) \quad, \quad 1 \leq \mathrm{i} \leq \mathrm{A}-1 \\
& \rho_{\mu}(\mathrm{A})=\frac{1}{\mathrm{~A}^{1 / 2}} \sum_{\mathrm{k}=1}^{\mathrm{A}} \mathrm{x}_{\mu}(\mathrm{k})=\mathrm{A}^{1 / 2} \mathrm{X}_{\mu}^{\mathrm{CM}} \\
& \pi_{\mu}(\mathrm{i})=\sum_{\mathrm{k}=1}^{\mathrm{A}} \mathrm{O}_{\mathrm{ik}} \mathrm{p}_{\mu}(\mathrm{k}) \quad, \quad 1 \leq \mathrm{i} \leq \mathrm{A}-1
\end{aligned}
$$




$$
\pi_{\mu}(\mathrm{A})=\frac{1}{\mathrm{~A}^{1 / 2}} \sum_{\mathrm{k}=1}^{\mathrm{A}} \mathrm{p}_{\mu}(\mathrm{k})=\frac{\mathrm{P}_{\mu}^{\mathrm{CM}}}{\mathrm{A}^{1 / 2}}
$$

Como O é uma matriz ortogonal, podemos inverter as relações acima sem dificuldade, obtendo:

$$
\begin{aligned}
& \mathrm{x}_{\mu}(\mathrm{i})=\sum_{\mathrm{k}=1}^{\mathrm{A}} \mathrm{O}_{\mathrm{ki}} \rho_{\mu}(\mathrm{k})=\sum_{\mathrm{k}=1}^{\mathrm{A}-1} \mathrm{O}_{\mathrm{ki}} \rho_{\mu}(\mathrm{k})+\mathrm{x}_{\mu}^{\mathrm{CM}} \\
& \mathrm{p}_{\mu}(\mathrm{i})=\sum_{\mathrm{k}=1}^{\mathrm{A}} \mathrm{O}_{\mathrm{ki}} \pi_{\mu}(\mathrm{k})=\sum_{\mathrm{k}=1}^{\mathrm{A}-1} \mathrm{O}_{\mathrm{ki}} \pi_{\mu}(\mathrm{k})+\frac{\mathrm{P}_{\mu}^{\mathrm{CM}}}{\mathrm{A}} .
\end{aligned}
$$

Usando as relações acima, podemos reescrever o operador criação e aniquilação de quanta de oscilador definido em (5) pelas seguintes expressões:

$$
\begin{aligned}
& \mathrm{a}_{\mu ; \mathrm{i}}^{+\mathrm{e}}\left(\mathrm{b}_{0}\right)=\sum_{\mathrm{k}=1}^{\mathrm{A}} \mathrm{O}_{\mathrm{ki}} \mathrm{a}_{\mu ; \mathrm{k}}^{+}\left(\mathrm{b}_{0}\right) \\
& \mathrm{a}_{\mu ; \mathrm{i}}^{\mathrm{e}}\left(\mathrm{b}_{0}\right)=\sum_{\mathrm{k}=1}^{\mathrm{A}} \mathrm{O}_{\mathrm{ki}} \mathrm{a}_{\mu ; \mathrm{k}}\left(\mathrm{b}_{0}\right)
\end{aligned}
$$

onde temos 


$$
\begin{aligned}
& \mathrm{a}_{\mu ; \mathrm{k}}^{+}\left(\mathrm{b}_{0}\right)=\frac{1}{\sqrt{2}}\left[\frac{\rho \mu^{(\mathrm{k})}}{\mathrm{b}_{0}}-\mathrm{i} \frac{\mathrm{b}_{0}}{\hbar} \pi_{\mu}(\mathrm{k})\right] ; \mathrm{k}=1, \ldots \mathrm{A}-1 \\
& a_{\mu ; \mathrm{A}}^{+}\left(\mathrm{b}_{0}\right)=\mathrm{a}_{\mu}^{+}{ }^{+}{ }_{\left(\mathrm{b}_{0}^{\mathrm{CM}}\right)}=\frac{1}{\sqrt{2}}\left[\frac{\mathrm{X}_{\mu}^{\mathrm{CM}}}{\mathrm{b}_{0}^{\mathrm{CM}}}-\mathrm{i} \frac{\mathrm{b}_{0}^{\mathrm{CM}}}{\hbar} \mathrm{P}_{\mu}^{\mathrm{CM}}\right]=\frac{1}{\mathrm{~A}^{1 / 2}} \sum_{\mathrm{k}=1}^{\mathrm{A}} \mathrm{a}_{\mu ; \mathrm{k}}^{+\mathrm{e}}\left(\mathrm{b}_{0}\right) \\
& \mathrm{a}_{\mu ; \mathrm{k}}\left(\mathrm{b}_{0}\right)=\left[\mathrm{a}_{\mu ; \mathrm{k}}^{+}\left(\mathrm{b}_{0}\right)\right]^{+}, \quad \mathrm{a}_{\mu}^{\mathrm{CM}}=\left[\mathrm{a}_{\mu}^{+}{ }^{+\mathrm{M}}\right]^{+} \quad \text { e } \quad \mathrm{b}_{0}^{\mathrm{CM}}=\frac{\mathrm{b}_{0}}{\sqrt{\mathrm{A}}} .
\end{aligned}
$$

Por meio da transformação ortogonal, O, definida em (12) e das relações (13) a hamiltoniana de oscilador de A-nucleons

$$
\mathrm{H}_{0}=\sum_{\mathrm{k}=1}^{\mathrm{A}} \sum_{\mu=1}^{3} \frac{\mathrm{p}_{\mu}(\mathrm{k})^{2}}{2 \mathrm{~m}}+\frac{1}{2} \mathrm{~m} \omega^{2} \mathrm{x}_{\mu}(\mathrm{k})^{2}
$$

se decompõe no termo $\mathrm{H}^{\text {int }}$ correspondente à hamiltoniana de oscilador dada em função de coordenadas intrínsecas e no termo $\mathrm{H}^{\mathrm{CM}}$ que corresponde à hamiltoniana de oscilador do centro de massa, isto é:

$$
\mathrm{H}_{0}=\mathrm{H}^{\mathrm{int}}+\mathrm{H}^{\mathrm{CM}}
$$

onde

$$
\begin{aligned}
& \mathrm{H}^{\text {int }}=\sum_{\mathrm{k}=1}^{\mathrm{A}-1} \sum_{\mu=1}^{3} \frac{\pi_{\mu}^{2}(\mathrm{k})}{2 \mathrm{~m}}+\frac{1}{2} \mathrm{~m} \omega^{2} \rho_{\mu}(\mathrm{k})^{2} \\
& \mathrm{H}^{\mathrm{CM}}=\sum_{\mu=1}^{3} \frac{\mathrm{P}^{\mathrm{CM}}}{2 \mathrm{Am}}+\frac{1}{2} \mathrm{Am} \omega^{2} \mathrm{X}_{\mu}^{\mathrm{CM}^{2}}
\end{aligned}
$$


e portanto segue que os operadores $\mathrm{a}_{\mu ; \mathrm{i}}^{+}\left(\mathrm{b}_{0}\right), \mathrm{a}_{\mu ; \mathrm{i}}\left(\mathrm{b}_{0}\right), 1 \leq \mathrm{i} \leq \mathrm{A}-1$ correspondem a operadores criação e aniquilação de quantas de oscilador referentes às coordenadas intrínsecas e que $a_{\mathrm{CM}}^{+}\left(\mathrm{b}_{0}^{\mathrm{CM}}\right)$ e $a_{\mathrm{CM}}\left(\mathrm{b}_{0}^{\mathrm{CM}}\right)$ correspondem aos operadores criação e aniquilição de quanta de oscilador do centro de massa.

A álgebra $\mathrm{sp}(3, \mathrm{R})$, definida em (6), através da transformação (14), pode ser decomposta da seguinte maneira:

$$
\begin{aligned}
& \mathrm{A}_{\mu \nu}^{+\mathrm{e}}=\mathrm{A}_{\mu \nu}^{+}+\mathrm{A}_{\mu \nu}^{+} \mathrm{CM} \\
& \mathrm{A}_{\mu \nu}^{\mathrm{e}}=\mathrm{A}_{\mu \nu}+\mathrm{A}_{\mu \nu}^{\mathrm{CM}} \\
& \mathrm{C}_{\mu \nu}^{\mathrm{e}}=\mathrm{C}_{\mu \nu}+\mathrm{C}_{\mu \nu}^{\mathrm{CM}}
\end{aligned}
$$

onde definimos

$$
\begin{aligned}
& \mathrm{A}_{\mu \nu}^{+}=\frac{1}{2} \sum_{\mathrm{k}=1}^{\mathrm{A}-1} \mathrm{a}_{\mu ; \mathrm{k}}^{+}\left(\mathrm{b}_{0}\right) \mathrm{a}_{\nu ; \mathrm{k}}^{+}\left(\mathrm{b}_{0}\right) \\
& \mathrm{A}_{\mu \nu}=\left[\mathrm{A}_{\mu \nu}^{+}\right]^{+} \\
& \mathrm{C}_{\mu \nu}=\frac{1}{2} \sum_{\mathrm{k}=1}^{\mathrm{A}-1} \mathrm{a}_{\mu ; \mathrm{k}}^{+}\left(\mathrm{b}_{0}\right) \mathrm{a}_{\nu ; \mathrm{k}}\left(\mathrm{b}_{0}\right)+\mathrm{a}_{\nu ; \mathrm{k}}\left(\mathrm{b}_{0}\right) \mathrm{a}_{\mu ; \mathrm{k}}^{+}\left(\mathrm{b}_{0}\right) .
\end{aligned}
$$

Os operadores acima formam uma álgebra $\mathrm{sp}(3, \mathrm{R})$ e vão gerar excitações intrínsecas.

De maneira análoga temos que 


$$
\begin{aligned}
& \left.\mathrm{A}_{\mu \nu}^{+} \mathrm{CM}=\frac{1}{2} \mathrm{a}_{\mu}^{+}{ }^{+}{ }_{\left(\mathrm{b}_{0}\right.}^{\mathrm{CM}}\right) \mathrm{a}_{\nu}^{+}{ }^{+}{ }_{\left(\mathrm{b}_{0}^{\mathrm{CM}}\right)}^{\mathrm{CM}} \\
& \mathrm{A}_{\mu \nu}^{\mathrm{CM}}=\left[\mathrm{A}_{\mu \nu}^{+} \mathrm{CM}\right]^{+} \\
& \mathrm{C}_{\mu \nu}^{\mathrm{CM}}=\frac{1}{2}\left[\mathrm{a}_{\mu}^{+}{ }^{+}{ }^{\mathrm{CM}}\left(\mathrm{b}_{0}^{\mathrm{CM}}\right) \mathrm{a}_{\nu}^{\mathrm{CM}}\left(\mathrm{b}_{0}^{\mathrm{CM}}\right)+\mathrm{a}_{\nu}^{\mathrm{CM}}\left(\mathrm{b}_{0}^{\mathrm{CM}}\right) \mathrm{a}_{\mu}^{+}{ }^{+}{ }^{\mathrm{CM}}\left(\mathrm{b}_{0}^{\mathrm{CM}}\right)\right] .
\end{aligned}
$$

Os operadores acima vão gerar excitações do centro de massa.

Os estados de peso mínimo de $\mathrm{Sp}(3, \mathrm{R})$, que iremos considerar, possuem a propriedade de serem aniquilados por $\mathrm{a}_{\mu}^{\mathrm{CM}}$ :

$$
\mathrm{a}_{\mu}^{\mathrm{CM}} \mid[\mathrm{f}] \mathrm{LW}>^{\mathrm{e}}=0 \quad ; \quad \forall \mu
$$

e portanto podem ser fatorados da seguinte maneira:

$$
\left|[\mathrm{f}] \mathrm{LW}>^{\mathrm{e}}=\right|[\mathrm{f}] \mathrm{LW}>\cdot \mid \phi_{0}^{\mathrm{CM}}>
$$

onde $\left|\phi_{0}^{\mathrm{CM}}\right\rangle$ corresponde ao estado fundamental do oscilador harmônico do centro de massa e $\mid[f] L W>$ é dado em função de coordenadas intrínsecas.

O estado |[f]LW> é estado de peso mínimo da álgebra $\operatorname{sp}(3, \mathrm{R})$ associada a excitações intrínsecas definida em (17), pois

$$
\begin{aligned}
& \mathrm{C}_{\mu \mu}\left|[\mathrm{f}] \mathrm{LW}>=\left[\mathrm{f}_{\mu}+\frac{(\mathrm{A}-1)}{2}\right]\right|[\mathrm{f}] \mathrm{LW}> \\
& \mathrm{C}_{\mu \nu} \mid[\mathrm{f}] \mathrm{LW}>=0 \quad \mu<\nu
\end{aligned}
$$




$$
\mathrm{A}_{\mu \nu} \mid[\mathrm{f}] \mathrm{LW}>=0, \forall \mu \mathrm{e} \nu .
$$

Portanto, através da ação dos operadores (17) no estado de peso mínimo |[f] $] \mathrm{LW}>$, obtemos os estados coletivos gerados pelo modelo simplético livre de contaminação devido às excitações espúrias do centro de massa. 


\section{Construção dos Estados de Peso Mínimo}

\subsection{Introdução}

No capítulo anterior vimos que uma base de uma representação irredutível de $\mathrm{Sp}(3, \mathrm{R})$ é determinada a partir de seu estado de peso mínimo. A seguir mostraremos como efetivamente construimos os estados de peso mínimo de $\mathrm{Sp}(3, \mathrm{R})$ adequados à descrição do núcleo ${ }^{12} \mathrm{C}$. Denominaremos as configurações com 4 nucleons na camada s e oito nucleons na camada p como "não excitadas" e as configurações envolvendo ocupação de outras camadas como "excitadas", onde sempre estamos nos referindo às funções de onda do oscilador harmônico.

\subsection{Configurações Não-Excitadas}

Nesta seção vamos discutir a determinação das representações irredutíveis de SU(3), bem como a construção de seus respectivos estados de peso mínimo para as configurações não excitadas do ${ }^{12} \mathrm{C}$. Pode-se mostrar ${ }^{(14)}$ que na camada $\mathrm{p}$ a classificação da parte orbital da função de onda de acordo com as representações irredutíveis de $\mathrm{U}(3)$ e das representações irredutíveis do grupo de permutações dos oito nucleons que ocupam esta camada é completamente equivalente.

Portanto na camada p a cada diagrama de Young associado a uma representação irredutível de $\mathrm{S}_{8}$ temos uma representação irredutível de $\mathrm{U}(3)$ correspondente. Como é bem conhecido, a redução $\mathrm{U}(3) \rightarrow \mathrm{SU}(3)$ ocorre sem multiplicidade e para obtermos as representações irredutíveis de $\mathrm{SU}(3)$ a partir de $\mathrm{U}(3)$ basta utilizarmos o procedimento de retirar todas as colunas de 3 elementos dos diagramas de Young associadas a $\mathrm{U}(3)$, na 
figura 1 mostramos um exemplo.

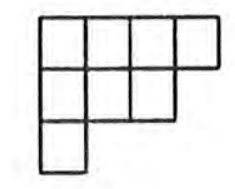

$[\mathrm{h}]=[431]$

representação de $\mathrm{U}(3)$ e $\mathrm{S}_{8}$

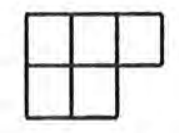

$\left[\mathrm{h}^{\prime}\right]=[32]$

representação de

$\mathrm{SU}(3)$

Fig. 3.1. Diagrama de Young associado à representação irredutível [h] de $\mathrm{S}_{8}$ e $\mathrm{U}(3)$ e o respectivo diagrama [h'] de $\mathrm{SU}(3)$ obtido pela retirada das colunas de 3 elementos.

Até este momento só nos referimos a simetria permutacional da parte orbital da função de onda, mas o nosso objetivo é construir uma função de onda totalmente antisimétrica a partir da simetria permutacional orbital $[\mathrm{g}]$ dos doze nucleons. O procedimento formal ${ }^{(14)}$ para construirmos a função de onda antisimétrica consiste em acoplar a função de onda orbital com simetria permutacional $[\mathrm{g}]$ com a função de onda no espaço de spin-isospin com simetria permutacional adjunta $[\tilde{g}]$, onde lembramos que $[\tilde{g}]$ é obtido a partir do diagrama $[\mathrm{g}]$ trocando suas linhas pelas suas colunas. Uma consequência imediata do procedimento descrito acima, é que os diagramas [g], da parte orbital da função de onda não podem ter mais do que quatro colunas, pois caso contrário isto implicaria em diagramas no espaço de spin-isospin com mais de quatro linhas e que envolveria antisimetrizar mais do que quatro partículas o que é evidentemente nulo, uma vez que só temos quatro estados distintos no espaço de spin-isospin.

Os quatro nucleons na camada s que temos ignorado até o momento, são inclú́dos 
de maneira trivial no diagrama $[\mathrm{g}]$ relativo à simetria permutacional orbital dos doze nucleons, pois a restrição de haver no máximo quatro colunas no diagrama da simetria orbital implica que apenas temos que acrescentar o número 4 ao diagrama [h] relativo à camada $\mathrm{p}$, isto é, se $[\mathrm{h}]=\left[\mathrm{h}_{1} \mathrm{~h}_{2} \mathrm{~h}_{3}\right]$ corresponde à simetria permutacional orbital dos oito nucleons na camada $\mathrm{p}$, então $[\mathrm{g}]=\left[4 \mathrm{~h}_{1} \mathrm{~h}_{2} \mathrm{~h}_{3}\right]$ corresponde à simetria permutacional orbital dos doze nucleons, onde é claro que $h_{1}+h_{2}+h_{3}=8$, e os rótulos de $S U(3)$ ficam inalterados.

A partir da discussão acima, já temos condições de obter todas as representações irredutíveis de $\mathrm{SU}(3)$ na camada $\mathrm{p}$ que tem interesse físico. Na tabela 3.1 mostramos os rótulos associados a estas representações, inclusive os rótulos de Elliott $(\lambda \mu)$, onde recordamos que tais rótulos são definidos a partir dos rótulos $[\mathrm{h}]$ de $\mathrm{U}(3)$ pela relação $\lambda=\mathrm{h}_{1}-\mathrm{h}_{2}, \mu=\mathrm{h}_{2}-\mathrm{h}_{3}$.

$\begin{array}{cccc}\mathrm{S}_{12} & \mathrm{U}(3) & \mathrm{SU}(3) & \mathrm{U}(4) \\ {[\mathrm{g}]} & {[\mathrm{h}]} & {\left[\mathrm{h}^{\prime}\right](\lambda \mu)} & {[\tilde{\mathrm{g}}]} \\ {[444]} & {[44]} & {[44](04)} & {[3333]} \\ {[4431]} & {[431]} & {[32](12)} & {[4332]} \\ {[4422]} & {[422]} & {[2](20)} & {[4422]} \\ {[4332]} & {[332]} & {[11](01)} & {[4431]}\end{array}$

Tab. 3.1. Diagramas de Young relativos à simetria permutacional orbital, rótulos de $\mathrm{U}(3), \quad \mathrm{SU}(3)$ e seus respectivos rótulos de Elliott associados às representações irredutíveis de $\mathrm{SU}(3)$ fisicamente aceitáveis para $0{ }^{12} \mathrm{C}$ na camada $\mathrm{p}$. Na última coluna temos os diagramas de simetria adjunta à simetria orbital referentes ao espaço de spin-isospin.

Para completar a classificação das representações irredutíveis de SU(3) lembramos que o diagrama $[\tilde{g}]$ associado à simetria permutacional dos nucleons no espaço de spin-isospin rotula as representações irredutíveis de $\mathrm{U}(4)$. Agora é conveniente 
rotularmos as representações irredutíveis de $\mathrm{U}(4)$ pela cadeia de subgrupos:

$$
\mathrm{U}(4) \rightarrow \mathrm{SU}(4) \rightarrow \mathrm{SU}^{\sigma}(2) \times \mathrm{SU}^{\tau}(2)
$$

onde $\mathrm{SU}^{\sigma}(2), \mathrm{SU}^{\tau}(2)$ correspondem aos subgrupos associados ao spin e isospin respectivamente. Na tabela 2 mostramos os supermultipletos de Wigner associados aos diagramas $[\mathrm{g}]$.

$\begin{array}{rll}{[\mathrm{g}]} & {[\tilde{g}]} & (\mathrm{ST}) \\ {[444]} & {[3333]} & (00) \\ {[4431]} & {[4332]} & (10)(01)(11) \\ {[4422]} & {[4422]} & (00)(02)(11)(20) \\ {[4332]} & {[4431]} & (21)(12)(11)(01)(10)\end{array}$

Tab. 3.2. Possíveis valores do spin e isospin, (ST), associados aos diagramas [g] associados à simetria permutacional orbital, que ocorrem na camada $\mathrm{p}$ para o ${ }^{12} \mathrm{C}$.

Vamos a seguir construir as representações irredutíveis de $\mathrm{SU}(3)$ de interesse para ${ }^{0}{ }^{12} \mathrm{C}$ onde todas as possibilidades fisicamente aceitáveis são obtidas na tabela 3.1.

Convém salientar, que na camada $\mathrm{p}$ os estados de peso mínimo de $\mathrm{SU}(3)$ são simultaneamente estados de peso mínimo de $\mathrm{Sp}(3, \mathrm{R})$. Isto é fácil ver a partir da definição de estado do peso mínimo dada no capítulo anterior, seção 2.3, lembrando que $\mathrm{A}_{\mu \nu}^{\mathrm{e}}$ destrói dois quanta de oscilador.

\subsubsection{Determinação da Representação Irredutf́vel [444] (04)}

A partir desta seção, estaremos sempre nos referindo às funções de onda do oscilador 
harmônico em uma base cartesiana. Para fixarmos a notação definimos:

$$
<\overrightarrow{\mathrm{x}} \mid \mathrm{n}_{1} \mathrm{n}_{2} \mathrm{n}_{3}>=\phi_{\mathrm{n}_{1} \mathrm{n}_{2} \mathrm{n}_{3}}(\overrightarrow{\mathrm{x}})=\prod_{i=1}^{3} \frac{1}{\sqrt{2^{\mathrm{n}_{\mathrm{i}}} \mathrm{n}_{\mathrm{i}} ! \pi^{1 / 2} \mathrm{~b}_{0}}} \mathrm{H}_{\mathrm{n}_{\mathrm{i}}}\left(\frac{\mathrm{x}_{\mathrm{i}}}{\mathrm{b}_{0}}\right) \mathrm{e}^{-\mathrm{x}_{\mathrm{i}}^{2} / 2 \mathrm{~b}_{0}^{2}}
$$

onde $b_{0}=\sqrt{\frac{\hbar}{m \omega}}$ corresponde ao comprimento do oscilador e $H_{n}(x)$ ao polinômio de Hermite de ordem $n$. Em geral usaremos a notação mais concisa, $\left(\mathrm{n}_{1} \mathrm{n}_{2} \mathrm{n}_{3}\right)^{\tau \sigma}$ para descrevermos a função de onda orbital acima, onde os índices superiores $\tau \sigma$ correspondem aos estados de isospin e spin respectivos; sendo

$$
\tau \sigma \in\{\mathrm{n}+, \mathrm{n}-, \mathrm{p}+, \mathrm{p}-\}
$$

e por convenção, identificamos as coordenadas cartesianas $(z, x, y)$ com as direções 1,2 e 3 respectivamente. O estado de peso mínimo de $\mathrm{SU}(3)$ é o estado, $\mid \mathrm{LW}>^{\mathrm{e}}$, onde

$$
\begin{aligned}
& {\left[\mathrm{C}_{11}^{\mathrm{e}}-\mathrm{C}_{22}^{\mathrm{e}}\right]\left|L W>^{\mathrm{e}}=\lambda\right| L W>^{\mathrm{e}}} \\
& {\left[\mathrm{C}_{22}^{\mathrm{e}}-\mathrm{C}_{33}^{\mathrm{e}}\right]\left|L W>^{\mathrm{e}}=\mu\right| L W>^{\mathrm{e}}} \\
& \mathrm{C}_{\mu \nu}^{\mathrm{e}} \mid L W>^{\mathrm{e}}=0 \quad \mu<\nu .
\end{aligned}
$$

A condição acima corresponde a colocarmos o maior número possível de quanta na direção 1 e respectivamente nas direções 2 e 3 mantendo a simetria orbital de $\mathrm{SU}(3)$.

Portanto se $(\lambda \mu)$ corresponde aos rótulos de Elliott, então: 


$$
\begin{aligned}
& \lambda=N_{1}-N_{2} \\
& \mu=N_{2}-N_{3}
\end{aligned}
$$

onde $\mathrm{N}_{\mathrm{i}}$ corresponde ao número de quanta na direção i do estado de peso mínimo. Como neste caso $(\lambda \mu)=(04)$ então:

$$
\begin{aligned}
& \lambda=0=\mathrm{N}_{1}-\mathrm{N}_{2} \\
& \mu=0=\mathrm{N}_{2}-\mathrm{N}_{3}
\end{aligned}
$$

e como temos 8 nucleons na camada p então:

$$
\mathrm{N}=\mathrm{N}_{1}+\mathrm{N}_{2}+\mathrm{N}_{3}=8
$$

onde $\mathrm{N}$ corresponde ao número total de quanta.

As equações 1 e 2 implicam que:

$$
\mathrm{N}_{1}=4, \mathrm{~N}_{2}=4, \mathrm{~N}_{3}=0
$$

e portanto usando a notação $\mid[\mathrm{g}](\lambda \mu) ;(\mathrm{ST})>_{\mathrm{LW}}^{\mathrm{e}}$ para o estado de peso mínimo, é imediato que neste caso:

$$
\mid[444](04) ; 00>{ }_{\mathrm{LW}}^{\mathrm{e}}=\frac{1}{\sqrt{12 !}} \operatorname{det}(000)^{4}(100)^{4}(010)^{4} .
$$

É trivial mostrarmos que a ação de $\mathrm{C}_{12}, \mathrm{C}_{13}, \mathrm{C}_{23}, \mathrm{~T}_{ \pm}, \mathrm{S}_{ \pm}$no estado acima é 
identicamente nula, onde os operadores $\mathrm{T}_{+}\left(\mathrm{T}_{-}\right), \mathrm{S}_{+}\left(\mathrm{S}_{-}\right)$correspondem aos operadores levantadores (abaixadores) no espaço de isospin e spin respectivamente. Observando as tabelas 1 e 2 notamos que o estado acima é o único estado de peso mínimo que pode ser construído com a simetria (04). Notando que:

$$
\mathrm{A}_{\mu \nu}^{\mathrm{e}} \mid[444](04) ; 00>{ }_{\mathrm{LW}}^{\mathrm{e}}=0 ; \forall \mu, \nu
$$

segue que o estado acima é estado de peso mínimo de $\mathrm{Sp}(3, \mathrm{R})$. Notemos que o estado (3.3) não é axialmente simétrico em relação ao eixo 1.

No cálculo usando o modelo $\mathrm{SU}(3)$, Elliott ${ }^{(2)}$ mostrou que os estados de uma representação irredutível, $(\lambda \mu)$, podem ser obtidos pela projeção em momento angular de seu estado de peso mínimo. No entanto, quando $\lambda<\mu$, mostrou que era mais conveniente projetar o estado de peso máximo de $\mathrm{SU}(3)$.

Recordemos que o estado de peso máximo de $\mathrm{SU}(3), \mid \mathrm{HW}>^{\mathrm{e}}$, é definido por:

$$
\begin{aligned}
& \mathrm{C}_{33}^{\mathrm{e}}-\mathrm{C}_{22}^{\mathrm{e}}\left|\mathrm{HW}>^{\mathrm{e}}=\lambda\right| H W>^{\mathrm{e}} \\
& \mathrm{C}_{22}^{\mathrm{e}}-\mathrm{C}_{11}^{\mathrm{e}}\left|\mathrm{HW}>^{\mathrm{e}}=\mu\right| \mathrm{HW}>^{\mathrm{e}} \\
& \mathrm{C}_{\mu \nu}^{\mathrm{e}} \mid \mathrm{HW}>^{\mathrm{e}}=0, \mu>\nu .
\end{aligned}
$$

Para conveniência futura, vamos a seguir obter o estado de peso máximo neste caso. Mais uma vez se $(\lambda \mu)$ são os rótulos de Elliott, então para o estado de peso máximo obtemos: 


$$
\lambda=0=\mathrm{N}_{3}-\mathrm{N}_{2}
$$

$$
\mu=4=\mathrm{N}_{2}-\mathrm{N}_{1}
$$

e também

$$
\mathrm{N}=\mathrm{N}_{1}+\mathrm{N}_{2}+\mathrm{N}_{3}=8
$$

As equações acima implicam que:

$$
\mathrm{N}_{1}=0, \mathrm{~N}_{2}=4, \mathrm{~N}_{3}=4
$$

e portanto, obtemos para o estado de peso máximo:

$$
\mid[444](04) ; 00>{ }_{\mathrm{HW}}^{\mathrm{e}}=\frac{1}{\sqrt{12 !}} \operatorname{det}(000)^{4}(010)^{4}(001)^{4}
$$

onde é trivial mostrar que a ação de $\mathrm{C}_{32}, \mathrm{C}_{21}, \mathrm{C}_{31}, \mathrm{~T}_{ \pm}, \mathrm{S}_{ \pm}$no estado acima é identicamente nula. De acordo com as tabelas 1 e 2, concluímos que o estado (5) é o único estado de peso máximo que pode ser construído com a simetria (04). Observemos que neste caso, o estado de peso máximo é axialmente simétrico em relação ao eixo 1.

\subsubsection{Determinação da Representação Irredutível [4431] (12)}

Analogamente a seção anterior vamos obter o estado de peso mínimo de SU(3), onde agora: 


$$
\begin{aligned}
& \mathrm{N}_{1}+\mathrm{N}_{2}+\mathrm{N}_{3}=8 \\
& \lambda=1=\mathrm{N}_{1}-\mathrm{N}_{2} \\
& \mu=2=\mathrm{N}_{2}-\mathrm{N}_{3} .
\end{aligned}
$$

O sistema de equações acima tem por solução:

$$
\mathrm{N}_{1}=4, \mathrm{~N}_{2}=3, \mathrm{~N}_{3}=1
$$

e portanto os estados de peso mínimo que respeitam a simetria (12), tem que ser gerados a partir dos determinantes:

$$
\operatorname{det}(000)^{4}(100)^{4}(010)^{\tau_{1} \sigma_{1}}(010)^{\tau_{2} \sigma_{2}}(010)^{\tau_{3} \sigma_{3}}(001)^{\tau_{4} \sigma_{4}}
$$

onde $\tau_{\mathbf{i}} \sigma_{\mathrm{i}}$ assumem todas as possibilidades que geram estados, (6), distintos. É fácil ver que existem 16 estados distintos em (6). A partir das tabelas 1 e 2 observamos que temos que obter os estados com spin e isospin:

$$
(\mathrm{ST})=\{(01),(10),(11)\} .
$$

Por inspeção, é fácil ver que existe um único estado com $\mathrm{S}=1, \mathrm{~S}_{\mathrm{z}}=1, \mathrm{~T}=1, \mathrm{~T}_{\mathrm{z}}=1$ onde $\mathrm{S}_{\mathrm{z}}, \mathrm{T}_{\mathrm{z}}$ correspondem às projeções do spin $\mathrm{e}$ isospin no eixo $\mathrm{z}$ e $\mathrm{t}_{\mathrm{z}}|\mathrm{p}>=-1 / 2| \mathrm{p}>, \mathrm{t}_{\mathrm{z}}|\mathrm{n}\rangle=+1 / 2 \mid \mathrm{n}>$. Este estado é dado por: 


$$
\begin{aligned}
& \mid[4431](12) ;(11) \mathrm{S}_{\mathrm{z}}=1 \mathrm{~T}_{\mathrm{z}}=1>_{\mathrm{LW}}^{\mathrm{e}}= \\
& =\frac{1}{\sqrt{12 !}} \operatorname{det}(000)^{4}(100)^{4}(010)^{\mathrm{n}+}(010)^{\mathrm{n}-}(010)^{\mathrm{p}+}(001)^{\mathrm{n}+}
\end{aligned}
$$

É imediato que o estado acima é aniquilado pelos abaixadores de $\mathrm{SU}(3), \mathrm{C}_{12}, \mathrm{C}_{13}, \mathrm{C}_{23}$. Pela ação do operador T- em (8) obtemos:

$$
\begin{aligned}
& \mid[4431](12) ;(11) \mathrm{S}_{\mathrm{z}}=1 \mathrm{~T}_{\mathrm{z}}=0>_{\mathrm{LW}}^{\mathrm{e}}=\frac{1}{2} . \\
& \cdot\left\{\frac{1}{\sqrt{12 !}} \operatorname{det}(000)^{4}(100)^{4}(010)^{\mathrm{n}+}(010)^{\mathrm{p}-}(010)^{\mathrm{p}+}(001)^{\mathrm{n}+}+\right. \\
& \left.+\frac{1}{\sqrt{12 !}} \operatorname{det}(000)^{4}(100)^{4}(010)^{\mathrm{n}+}(010)^{\mathrm{n}-}(010)^{\mathrm{p}+}(001)^{\mathrm{p}+}\right\} .
\end{aligned}
$$

Construindo o estado ortogonal ao estado acima obtemos:

$$
\begin{aligned}
& \mid[4431](12) ;(10) \mathrm{S}_{\mathrm{z}}=1 \mathrm{~T}_{\mathrm{z}}=0>_{\mathrm{LW}}^{\mathrm{e}}=\frac{1}{2} . \\
& \cdot\left\{\frac{1}{\sqrt{12 !}} \operatorname{det}(000)^{4}(100)^{4}(010)^{\mathrm{n}+}(010)^{\mathrm{p}-}(010)^{\mathrm{p}+}(001)^{\mathrm{n}+}-\right. \\
& \left.-\frac{1}{\sqrt{12 !}} \operatorname{det}(000)^{4}(100)^{4}(010)^{\mathrm{n}+}(010)^{\mathrm{n}-}(010)^{\mathrm{p}+}(001)^{\mathrm{p}+}\right\} .
\end{aligned}
$$

Analisando o supermultipleto dado em (7), vemos que o estado acima pertence ao multipleto (10), uma vez que é aniquilado por $\mathrm{C}_{\mathrm{ij}}, \mathrm{i}<\mathrm{j}$, tem $\mathrm{S}_{\mathrm{z}}=1, \mathrm{~T}_{\mathrm{z}}=0$ e é aniquilado por $T_{+}$e $T_{-}$. 
A partir da ação de S. em (8) obtemos

$$
\begin{aligned}
& \mid[4431](12) ;(11) \mathrm{S}_{\mathrm{z}}=0 \mathrm{~T}_{\mathrm{z}}=1>_{\mathrm{LW}}^{\mathrm{e}}=\frac{1}{2} . \\
& \cdot\left\{\frac{1}{\sqrt{12 !}} \operatorname{det}(000)^{4}(100)^{4}(010)^{\mathrm{n}+}(010)^{\mathrm{n}-}(010)^{\mathrm{p}-}(001)^{\mathrm{n}+}+\right. \\
& \left.+\frac{1}{\sqrt{12 !}} \operatorname{det}(000)^{4}(100)^{4}(010)^{\mathrm{n}+}(010)^{\mathrm{n}-}(010)^{\mathrm{P}+}(001)^{\mathrm{n}-}\right\} .
\end{aligned}
$$

O mesmo raciocínio usado acima, nos permite concluir que o estado ortogonal ao estado acima pertence ao multipleto (01), e este estado é dado por:

$$
\begin{aligned}
& \mid[4431](12) ;(01) \mathrm{S}_{\mathrm{z}}=0 \mathrm{~T}_{\mathrm{z}}=1>_{\mathrm{LW}}^{\mathrm{e}}=\frac{1}{2} . \\
& \cdot\left\{\frac{1}{\sqrt{12 !}} \operatorname{det}(000)^{4}(100)^{4}(010)^{\mathrm{n}+}(010)^{\mathrm{n}-}(010)^{\mathrm{p}-}(001)^{\mathrm{n}+}-\right. \\
& \left.-\frac{1}{\sqrt{12 !}} \operatorname{det}(000)^{4}(100)^{4}(010)^{\mathrm{n}+}(010)^{\mathrm{n}-}(010)^{\mathrm{p}+}(001)^{\mathrm{n}-}\right\} .
\end{aligned}
$$

Pela ação repetida de $S_{ \pm}, T_{ \pm}$nos estados (8), (10) e (11) obtemos todos os 15 estados do supermultipleto de $\mathrm{SU}(4)$, dados em (7), associados à representação irredutível $(\lambda \mu)=(12)$ de $\mathrm{SU}(3)$. 


\subsubsection{Determinação da representação Irredutível [4422] (20)}

Vamos então construir o estado de peso mínimo, com

$$
\begin{aligned}
& \lambda=\mathrm{N}_{1}-\mathrm{N}_{2}=2 \\
& \mu=\mathrm{N}_{2}-\mathrm{N}_{3}=0
\end{aligned}
$$

e analogamente às seções anteriores obtemos:

$$
\begin{aligned}
& N=8=N_{1}+N_{2}+N_{3} \\
& \lambda=2=N_{1}-N_{2} \\
& \mu=0=N_{2}-N_{3} .
\end{aligned}
$$

O sistema acima tem a seguinte solução:

$$
\mathrm{N}_{1}=4, \mathrm{~N}_{2}=\mathrm{N}_{3}=2 \text {. }
$$

Os estados com simetria $(\lambda \mu)=(20)$ e com (ST) bem definidos, devem ser construídos a partir dos 36 determinantes distintos, que apresentamos a seguir:

$$
\operatorname{det}(000)^{4}(100)^{4}(010)^{\tau_{1} \sigma_{1}}(010)^{\tau_{2} \sigma_{2}}(001)^{\tau_{3} \sigma_{3}}(001)^{\tau_{3} \sigma_{3}} .
$$


Analogamente à seção 3.4, por inspeção construímos o único estado com $\mathrm{T}=2, \mathrm{~T}_{\mathrm{z}}=2$, $\mathrm{S}=0, \mathrm{~S}_{\mathrm{z}}=0$

$\mid[4422](20) ;(02) \mathrm{S}_{\mathrm{z}}=0 \mathrm{~T}_{\mathrm{z}}=2>>_{L W}^{\mathrm{e}}=\frac{1}{\sqrt{12 !}} \operatorname{det}(000)^{4}(100)^{4}(010)^{\mathrm{n}+}(010)^{\mathrm{n}-}(001)^{\mathrm{n}+}(001)^{\mathrm{n}-}$

e o único estado com $\mathrm{T}=0, \mathrm{~T}_{\mathrm{z}}=0, \mathrm{~S}=2, \mathrm{~S}_{\mathrm{z}}=2$

$\mid[4422](20) ;(20) \mathrm{S}_{\mathrm{z}}=2 \mathrm{~T}_{\mathrm{z}}=0>_{\mathrm{LW}}^{\mathrm{e}}=\frac{1}{\sqrt{12 !}} \operatorname{det}(000)^{4}(100)^{4}(010)^{\mathrm{n}+}(010)^{\mathrm{p}+}(001)^{\mathrm{n}+}(001)^{\mathrm{p}+}$

A partir da eq. 3.8 notamos que existem dois determinantes com $\mathrm{T}=1, \mathrm{~T}_{\mathrm{z}}=1, \mathrm{~S}=1$, $\mathrm{S}_{\mathrm{z}}=1$ e com essa configuração, isto é:

$$
\begin{aligned}
& \operatorname{det}(000)^{4}(100)^{4}(010)^{\mathrm{n}+}(010)^{\mathrm{p}+}(001)^{\mathrm{n}+}(001)^{\mathrm{n}-} \\
& \operatorname{det}(000)^{4}(100)^{4}(010)^{\mathrm{n}+}(010)^{\mathrm{n}-}(001)^{\mathrm{n}+}(001)^{\mathrm{p}+}
\end{aligned}
$$

para determinarmos qual a combinação dos determinantes acima que corresponde ao estado de peso mínimo de $\mathrm{SU}(3)$, usamos a propriedade de que tal estado deve ser aniquilado pelos abaixadores de $\mathrm{SU}(3)$ e portanto obtemos: 


$$
\begin{aligned}
& \mid[4422](20) ;(11) \mathrm{S}_{\mathrm{z}}=1 \mathrm{~T}_{\mathrm{z}}=1>_{\mathrm{LW}}^{\mathrm{e}}=\frac{1}{\sqrt{2}} . \\
& \cdot\left\{\frac{1}{\sqrt{12 !}} \operatorname{det}(000)^{4}(100)^{4}(010)^{\mathrm{n}+}(010)^{\mathrm{p}+}(001)^{\mathrm{n}+}(001)^{\mathrm{n}-}+\right. \\
& \left.+\frac{1}{\sqrt{12 !}} \operatorname{det}(000)^{4}(100)^{4}(010)^{\mathrm{n}+}(010)^{\mathrm{n}-}(001)^{\mathrm{n}+}(001)^{\mathrm{p}+}\right\} .
\end{aligned}
$$

Notemos que o estado acima é ortogonal ao estado da representação irredutível (12)(S=1 $\mathrm{T}=1$ ), eq.(8), de mesma configuração.

Aplicando duas vezes o operador T - no estado (13) obtemos:

$$
\begin{aligned}
& \mid[4422](20) ;(02) \mathrm{S}_{\mathrm{z}}=0 \quad \mathrm{~T}_{\mathrm{z}}=0>_{\mathrm{LW}}^{\mathrm{e}}=\frac{1}{\sqrt{6}} \cdot \frac{1}{\sqrt{12 !}} \cdot \\
& \cdot\left\{\operatorname{det}(000)^{4}(100)^{4}(010)^{\mathrm{p}+}(010)^{\mathrm{p}-}(001)^{\mathrm{n}+}(001)^{\mathrm{n}-}+\right. \\
& +\operatorname{det}(000)^{4}(100)^{4}(010)^{\mathrm{n}+}(010)^{\mathrm{p}-}(001)^{\mathrm{n}+}(001)^{\mathrm{p}-}- \\
& -\operatorname{det}(000)^{4}(100)^{4}(010)^{\mathrm{n}+}(010)^{\mathrm{p}-}(001)^{\mathrm{n}-}(001)^{\mathrm{p}+}- \\
& -\operatorname{det}(000)^{4}(100)^{4}(010)^{\mathrm{n}-}(010)^{\mathrm{p}+}(001)^{\mathrm{n}+}(001)^{\mathrm{p}-}+ \\
& +\operatorname{det}(000)^{4}(100)^{4}(010)^{\mathrm{n}-}(010)^{\mathrm{p}+}(001)^{\mathrm{n}-}(001)^{\mathrm{p}+}+ \\
& \left.+\operatorname{det}(000)^{4}(100)^{4}(010)^{\mathrm{n}+}(010)^{\mathrm{n}-}(001)^{\mathrm{p}+}(001)^{\mathrm{p}-}\right\}
\end{aligned}
$$


Aplicando duas vezes o operador S. no estado (14) obtemos:

$$
\begin{aligned}
& \mid[4422](20) ;(20) \mathrm{S}_{\mathrm{z}}=0 \quad \mathrm{~T}_{\mathrm{z}}=0>_{\mathrm{LW}}^{\mathrm{e}}=\frac{1}{\sqrt{6}} \cdot \frac{1}{\sqrt{12 !}} \cdot \\
& \cdot\left\{\operatorname{det}(000)^{4}(100)^{4}(010)^{\mathrm{n}-}(010)^{\mathrm{p}-}(001)^{\mathrm{n}+}(001)^{\mathrm{p}+}+\right. \\
& +\operatorname{det}(000)^{4}(100)^{4}(010)^{\mathrm{n}+}(010)^{\mathrm{p}-}(001)^{\mathrm{n}+}(001)^{\mathrm{p}-}+ \\
& +\operatorname{det}(000)^{4}(100)^{4}(010)^{\mathrm{n}+}(010)^{\mathrm{p}-}(001)^{\mathrm{n}-}(001)^{\mathrm{p}+}+ \\
& +\operatorname{det}(000)^{4}(100)^{4}(010)^{\mathrm{n}-}(010)^{\mathrm{p}+}(001)^{\mathrm{n}+}(001)^{\mathrm{p}-}+ \\
& +\operatorname{det}(000)^{4}(100)^{4}(010)^{\mathrm{n}-}(010)^{\mathrm{p}+}(001)^{\mathrm{n}-}(001)^{\mathrm{p}+}+ \\
& \left.+\operatorname{det}(000)^{4}(100)^{4}(010)^{\mathrm{n}+}(010)^{\mathrm{p}+}(001)^{\mathrm{n}-}(001)^{\mathrm{p}-}\right\}
\end{aligned}
$$

Através da ação de T. e S. no estado (15) obtemos:

$$
\begin{aligned}
& \mid[4422](20) ;(11) \mathrm{S}_{\mathrm{z}}=0 \quad \mathrm{~T}_{\mathrm{z}}=0>_{\mathrm{LW}}^{\mathrm{e}}=\frac{1}{\sqrt{2}} \cdot \\
& \cdot\left\{\frac{1}{\sqrt{12 !}} \operatorname{det}(000)^{4}(100)^{4}(010)^{\mathrm{n}+}(010)^{\mathrm{p}-}(001)^{\mathrm{n}+}(001)^{\mathrm{p}-}-\right. \\
& \left.-\frac{1}{\sqrt{12 !}} \operatorname{det}(000)^{4}(100)^{4}(010)^{\mathrm{n}-}(010)^{\mathrm{p}+}(001)^{\mathrm{n}-}(001)^{\mathrm{p}+}\right\} .
\end{aligned}
$$


Observando as tabelas 1 e 2, vemos que para finalizar a construção dos estados de peso mínimo $(\lambda \mu)=(20)$, ainda precisamos obter o estado com $\mathrm{S}=0, \mathrm{~T}=0$. Como este estado é ortogonal aos estados (16), (17), (18), e é aniquilado pelos operadores $\mathrm{T}_{ \pm}, \mathrm{S}_{ \pm}$, $\mathrm{C}_{23}, \mathrm{C}_{12}, \mathrm{C}_{13}$ temos condições suficientes para determinar a combinação linear dos oito determinantes distintos, dados em (12) que possuem $\mathrm{S}_{\mathrm{z}}=0, \mathrm{~T}_{\mathrm{z}}=0$. Como resultado obtemos:

$$
\begin{aligned}
& \mid[4422](20) ;(00)>\underset{L W}{\mathrm{e}}=\frac{1}{\sqrt{6}} \cdot \frac{1}{\sqrt{12 !}} \cdot \\
& \cdot\left\{\operatorname{det}(000)^{4}(100)^{4}(010)^{\mathrm{n}_{+}}(010)^{\mathrm{n}-}(001)^{\mathrm{p}+}(001)^{\mathrm{p}-}+\right. \\
& +\operatorname{det}(000)^{4}(100)^{4}(010)^{\mathrm{p}+}(010)^{\mathrm{p}-}(001)^{\mathrm{n}+}(001)^{\mathrm{n}-}+ \\
& +\operatorname{det}(000)^{4}(100)^{4}(010)^{\mathrm{n}-}(010)^{\mathrm{p}-}(001)^{\mathrm{n}_{+}}(001)^{\mathrm{p}+}+ \\
& +\operatorname{det}(000)^{4}(100)^{4}(010)^{\mathrm{n}+}(010)^{\mathrm{p}+}(001)^{\mathrm{n}-}(001)^{\mathrm{p}-}- \\
& -\operatorname{det}(000)^{4}(100)^{4}(010)^{\mathrm{n}-}(010)^{\mathrm{p}+}(001)^{\mathrm{n}-}(001)^{\mathrm{p}+}- \\
& \left.-\operatorname{det}(000)^{4}(100)^{4}(010)^{\mathrm{n}_{+}}(010)^{\mathrm{p}-}(001)^{\mathrm{n}_{+}}(001)^{\mathrm{p}-}\right\}
\end{aligned}
$$




\subsection{Construção dos Estados de Peso Mínimo Excitados}

Na seção anterior, discutimos de uma maneira razoavelmente completa a contrução dos estados de peso mínimo de $\mathrm{SU}(3)$ e $\mathrm{Sp}(3, \mathrm{R})$, dentro da camada p com bom $\mathrm{S}$ e $\mathrm{T}$ que eram adequados à descrição do ${ }^{12} \mathrm{C}$ e denominamos tais estados de não-excitados. A seguir contruiremos os estados de peso mínimo de $\mathrm{Sp}(3, \mathrm{R})$ e $\mathrm{SU}(3)$ excitados. Nos restringiremos aos casos que serão relevantes à análise dos estados do ${ }^{12} \mathrm{C}$ que pretendemos estudar nos próximos capítulos.

\subsubsection{Estado Duas Vezes Excitado}

De acordo com a referência 15 , a representação de $\mathrm{SU}(3)$ duas vezes excitada que é esperada ser de energia mais baixa é a representação com rótulos $[g](\lambda \mu)=[444](62)$ e que possui dois nucleons na camada sd.

O estado de peso mínimo desta representação irredutível tem:

$$
\begin{aligned}
& \mathrm{N}_{1}+\mathrm{N}_{2}+\mathrm{N}_{3}=10 \\
& \lambda=6=\mathrm{N}_{1}-\mathrm{N}_{2} \\
& \mu=2=\mathrm{N}_{2}-\mathrm{N}_{3}
\end{aligned}
$$

e portanto $\mathrm{N}_{1}=8, \mathrm{~N}_{2}=2, \mathrm{~N}_{3}=0$.

Para construirmos o estado de peso mínimo com os números de quanta acima, vamos utilizar um procedimento que irá facilitar em muito o nosso trabalho. Neste caso é fácil ver que a configuração do estado de peso mínimo é dada por: 


$$
(000)^{4}(100)^{4}(010)^{2}(200)^{2}
$$

para determinarmos o estado com $\mathrm{S}=0, \mathrm{~T}=0$, e simetria permutacional orbital [444] com a configuração acima, basta considerarmos o estado com a configuração

$$
(000)^{4}(100)^{4}(010)^{2}(001)^{2}
$$

da representação [444](04); $(\mathrm{S}=0 \mathrm{~T}=0)$, que obtemos a partir da expresão (3.3).

Como resultado do procedimento descrito acima obtemos:

$$
\begin{aligned}
& \mid[444](62) ;(00)>\underset{L W}{e}=\frac{1}{\sqrt{6}} \cdot \frac{1}{\sqrt{12 !}} \cdot \\
& \cdot\left\{\operatorname{det}(000)^{4}(100)^{4}(010)^{\mathrm{n}+}(010)^{\mathrm{n}-}(200)^{\mathrm{p}+}(200)^{\mathrm{p}-}-\right. \\
& -\operatorname{det}(000)^{4}(100)^{4}(010)^{\mathrm{n}+}(010)^{\mathrm{p}+}(200)^{\mathrm{n}-}(200)^{\mathrm{p}-}+ \\
& +\operatorname{det}(000)^{4}(100)^{4}(010)^{\mathrm{n}+}(010)^{\mathrm{p}-}(200)^{\mathrm{n}-}(200)^{\mathrm{p}+}- \\
& -\operatorname{det}(000)^{4}(100)^{4}(010)^{\mathrm{n}-}(010)^{\mathrm{p}-}(200)^{\mathrm{n}+}(200)^{\mathrm{p}+}+ \\
& +\operatorname{det}(000)^{4}(100)^{4}(010)^{\mathrm{p}+}(010)^{\mathrm{p}-}(200)^{\mathrm{n}+}(200)^{\mathrm{n}-}+ \\
& \left.+\operatorname{det}(000)^{4}(100)^{4}(010)^{\mathrm{n}-}(010)^{\mathrm{p}+}(200)^{\mathrm{n}+}(200)^{\mathrm{p}-}\right\}
\end{aligned}
$$


É fácil mostrar pelo cálculo explícito, que o estado acima é aniquilado pelos operadores abaixadores de $\mathrm{SU}(3)$ e por $\mathrm{T}_{ \pm}$e $\mathrm{S}_{ \pm}$. O estado acima também é estado de peso mínimo de $\operatorname{Sp}(3, \mathrm{R})$ pois é fácil ver que ele é aniquilado por $\mathrm{A}_{\mu \nu}^{\mathrm{e}}$.

\subsubsection{Estado Quatro Vezes Excitado}

Nesta seção vamos nos guiar pelas referências 10 e 15, a fim de escolhermos a representação irredutível de $\mathrm{SU}(3)$ quatro vezes excitada que é esperada ser de mais baixa energia. A análise feita nas referências citadas acima, resulta na escolha da representação com rótulos [444](120), que possui 4 nucleons na camada sd.

O estado de peso mínimo desta representação irredutível tem

$$
\begin{aligned}
& \mathrm{N}_{1}+\mathrm{N}_{2}+\mathrm{N}_{3}=12 \\
& \lambda=12=\mathrm{N}_{1}-\mathrm{N}_{2} \\
& \mu=0=\mathrm{N}_{2}-\mathrm{N}_{3}
\end{aligned}
$$

e portanto obtemos $\mathrm{N}_{1}=12, \mathrm{~N}_{2}=0, \mathrm{~N}_{3}=0$.

O estado de peso mínimo da representação [444](120) é dado por:

$$
\mid[444](120) ;(00)>_{\mathrm{LW}}^{\mathrm{e}}=\frac{1}{\sqrt{12 !}} \operatorname{det}(000)^{4}(100)^{4}(200)^{4}
$$


Um cálculo direto mostra que o estado acima é aniquilado pelos abaixadores de $\mathrm{SU}(3)$ e por $\mathrm{T}_{ \pm}, \mathrm{S}_{ \pm}$. É fácil mostrar que o estado acima é estado de peso mínimo de $\mathrm{Sp}(3, \mathrm{R})$. 


\section{Aplicação do Modelo Simplético à Descrição dos Níveis de Baixa Energia do ${ }^{12} \mathrm{C}$}

\subsection{Introdução}

No capítulo anterior mostramos como podemos obter os estados de peso mínimo de $\mathrm{Sp}(3, \mathrm{R})$ que são adequados à descrição do núcleo ${ }^{12} \mathrm{C}$. Naturalmente não vamos considerar a totalidade destes estados na construção do subespaço coletivo que associaremos ao ${ }^{12} \mathrm{C}$, uma vez que seria impraticável do ponto de vista algébrico e computacional. Também não vamos realizar um cálculo do tipo $\mathrm{Sp}(3, \mathrm{R})$ completo devido a dificuldades de caráter técnico. Na seção 2, consideraremos os dois submodelos do SCM que iremos efetivamente utilizar. Na seção 3 , discutiremos as propriedades dos estados de peso mínimo dos submodelos $\mathrm{Sp}(1, \mathrm{R})$ do $\mathrm{SCM}$, que em princípio, serão suficientes para um tratamento adequado dos estados de baixa energia do ${ }^{12} \mathrm{C}$. Estes estados vão determinar o subespaço coletivo gerado pelo modelo.

Como mostraremos, para os operadores de interesse físico pertencentes à algebra $\mathrm{sp}(3, \mathrm{R})$, podemos obter expressões analíticas simples para os seus elementos de matriz na base de estados gerados pelo modelo. Com relação aos operadores que não pertencem à álgebra $s p(3, R)$, vamos utilizar a técnica conhecida por método da função geratriz, MFG, que como veremos tem a grande vantagem de reduzir o cálculo de operadores entre funções de onda complicadas em derivadas de expressões relativamente simples. 


\subsection{Submodelos do Modelo Simplético}

Como mencionamos anteriormente, devido a dificuldades técnicas e computacionais, vamos utilizar apenas submodelos do SCM nos cálculos que realizaremos. A seguir descreveremos os dois submodelos ${ }^{(13)}$ que vamos efetivamente utilizar. A notação usada é a mesma que definimos na capítulo 2 .

\subsubsection{Modelo $\mathrm{Sp}_{\perp}(1, \mathrm{R})$}

A álgebra $\mathrm{sp}_{\perp}(1, \mathrm{R})$ é gerada por meio dos seguintes operadores:

$$
\begin{aligned}
& A_{\perp}^{+}\left(b_{0}\right)=A_{22}^{+}\left(b_{0}\right)+A_{33}^{+}\left(b_{0}\right) \\
& A_{\perp}\left(b_{0}\right)=A_{22}\left(b_{0}\right)+A_{33}\left(b_{0}\right) \\
& A_{0_{\perp}}\left(b_{0}\right)=\frac{1}{2} C_{22}\left(b_{0}\right)+\frac{1}{2} C_{33}\left(b_{0}\right)
\end{aligned}
$$

É fácil mostrar que os operadores acima satisfazem as seguintes relações de comutação:

$$
\begin{aligned}
& {\left[\mathrm{A}_{0_{\perp}}, \mathrm{A}_{\perp}^{+}\right]=\mathrm{A}_{\perp}^{+}} \\
& {\left[\mathrm{A}_{0_{\perp}}, \mathrm{A}_{\perp}\right]=-\mathrm{A}_{\perp}} \\
& {\left[\mathrm{A}_{\perp}^{+}, \mathrm{A}_{\perp}\right]=-2 \mathrm{~A}_{0_{\perp}} .}
\end{aligned}
$$


As relações acima determinam a álgebra $s p(1, R)$, que naturalmente é uma subálgebra de $\operatorname{sp}(3, R)$.

O estado de peso mínimo da álgebra acima é definido como sendo o estado, $\mid 0>$, que é aniquilado por $\mathrm{A}_{\perp}$ e é autoestado de $\mathrm{A}_{0_{\perp}}$, isto é:

$$
\begin{aligned}
& \mathrm{A}_{0_{\perp}}\left|0>=\mathrm{k}_{\perp}\right| 0> \\
& \mathrm{A}_{\perp} \mid 0>=0 .
\end{aligned}
$$

Os estados de base da representação irredutível, com rótulo $\mathrm{k}_{\perp}$, de $\operatorname{Sp}(1, \mathrm{R})$ são dados por:

$$
\left|n>=\left[\mathrm{A}_{\perp}^{+}\right]^{\mathrm{n}}\right| 0>\cdot\left[\frac{\Gamma\left(2 \mathrm{k}_{\perp}\right)}{\Gamma(\mathrm{n}+1) \Gamma\left(\mathrm{n}+2 \mathrm{k}_{\perp}\right)}\right]^{1 / 2}, \quad \mathrm{n}=0,1, \ldots
$$

Os estados acima correspondem a vibrações no plano perpendicular ao eixo de simetria axial, onde estamos supondo o estado, $|0\rangle$, axialmente simétrico. O operador:

$$
\mathrm{H}_{\perp}=2 \hbar \omega \mathrm{A}_{0_{\perp}}
$$

corresponde à hamiltoniana de oscilador dada em coordenadas intrínsecas, associada a vibrações no plano perpendicular ao eixo 1 e temos:

$$
\mathrm{H}_{\perp}\left|\mathrm{n}>=\left(\mathrm{E}_{0}+2 \mathrm{n} \hbar \omega\right)\right| \mathrm{n}>
$$

e portanto, os estados, $\mid n>$, podem ser interpretados como estados vibracionais de n-fonons transversais. 
O modelo $\operatorname{Sp}_{\perp}(1, R)$ é utilizado na maioria das vezes, na descrição de núcleos oblatos. As oscilações geradas pelo modelo são obtidas acrescentando-se quanta de oscilador no plano perpendicular ao eixo de simetria e o estado de peso mínimo de $\mathrm{Sp}_{\perp}(1, \mathrm{R})$ em geral, também é estado de peso máximo de $\mathrm{SU}(3)$ com rótulos de Elliott $(0 \mu)$. O subespaço coletivo gerado pelo modelo é obtido pela projeção em momento angular dos estados (3):

$$
\left|\mathrm{n}, \mathrm{LM} 0>=\mathrm{P}_{\mathrm{M} 0}^{\mathrm{L}}\right| \mathrm{n}>\quad, \quad \mathrm{n}=0,1, \ldots
$$

onde o projetor $\mathrm{P}_{\mathrm{MK}}^{\mathrm{L}}$, definido pelas expressões (25), só projeta estados com $\mathrm{K}=0$, devido à simetria axial.

A partir da análise feita no apêndice 1 , conclúmos que os valores de L acima são dados pela regra:

$$
\mathrm{L}=\mu+2 \mathrm{n}, \mu+2 \mathrm{n}-2, \ldots, 1 \text { ou } 0
$$

e satisfazem a restrição $\mathrm{L} \leq \mu+2 \mathrm{n}$.

Os estados (4) formam uma base ortogonal, uma vez que são autoestados da hamiltoniana do oscilador harmônico esférico, dada em coordenadas intrínsecas, com energia de excitação $2 n \hbar \omega$. 


\subsubsection{Modelo $\mathrm{Sp}_{11}(1, \mathrm{R})$}

Neste caso a álgebra $\mathrm{sp}_{\|}(1, \mathrm{R})$ é gerada pelos seguintes operadores:

$$
\begin{aligned}
& \mathrm{A}_{11}^{+}\left(\mathrm{b}_{0}\right)=\mathrm{A}_{11}^{+}\left(\mathrm{b}_{0}\right) \\
& \mathrm{A}_{11}\left(\mathrm{~b}_{0}\right)=\mathrm{A}_{11}\left(\mathrm{~b}_{0}\right) \\
& \mathrm{A}_{011}\left(\mathrm{~b}_{0}\right)=\frac{1}{2} \mathrm{C}_{11}\left(\mathrm{~b}_{0}\right)
\end{aligned}
$$

é fácil mostrar que por comutação temos:

$$
\begin{aligned}
& {\left[A_{011}, A_{11}^{+}\right]=A_{11}^{+}} \\
& {\left[A_{0 "}, A_{11}\right]=-A_{11}} \\
& {\left[A_{11}^{+}, A_{11}\right]=-2 A_{0 "}}
\end{aligned}
$$

e portanto os operadores (5) geram uma álgebra $\mathrm{sp}(1, \mathrm{R})$.

O estado de peso mínimo da álgebra acima é definido como sendo o estado $|0\rangle$, que é aniquilado por $A_{11}$ e é autoestado de $A_{011}$, isto é:

$$
\begin{aligned}
& \mathrm{A}_{011}\left|0>=\mathrm{k}_{11}\right| 0> \\
& \mathrm{A}_{11} \mid 0>=0 .
\end{aligned}
$$


Os estados de base da representação irredutível de $\operatorname{Sp}_{11}(1, R)$, com rótulo $k_{\| 1}$, são dados por:

$$
\left|\mathrm{n}>=\left[\mathrm{A}_{11}^{+}\right]^{\mathrm{n}}\right| 0>\cdot\left[\frac{\Gamma\left(2 \mathrm{k}_{\mathrm{\prime \prime}}\right)}{\Gamma(\mathrm{n}+1) \Gamma\left(\mathrm{n}+2 \mathrm{k}_{\mathrm{\prime l}}\right)}\right]^{1 / 2}, \quad \mathrm{n}=0,1, \ldots
$$

Os estados acima correspondem a vibrações na direção do eixo 1. O operador

$$
\mathrm{H}_{11}=2 \hbar \omega \mathrm{A}_{0 \prime \prime}
$$

corresponde à hamiltoniana de oscilador, dada em coordenadas intrínsecas, associada a vibrações na direção 1 e como

$$
\mathrm{H}_{11}\left|\mathrm{n}>=\left(\mathrm{E}_{0}+2 \mathrm{n} \hbar \omega\right)\right| \mathrm{n}>
$$

podemos interpretar os estados $|\mathrm{n}\rangle$, como estados vibracionais longitudinais de $\mathrm{n}$-fonons.

Em todos os casos que iremos considerar, o estado $|0\rangle$, vai ser estado de peso mínimo de $\mathrm{Sp}_{\text {II }}(1, \mathrm{R}), \mathrm{Sp}(3, \mathrm{R})$ e $\mathrm{SU}(3)$ com rótulos de Elliott $(\lambda \mu)$. Como o estado $\mid 0>$ é estado de peso mínimo de $\mathrm{SU}(3)$ e os abaixadores de $\mathrm{su}(3)$ comutam com $\mathrm{A}_{11}^{+}$, isto é:

$$
\left[\mathrm{C}_{\mathrm{ij}}, \mathrm{A}_{11}^{+}\right]=0, \mathrm{i}<\mathrm{j}
$$

é fácil mostrar que $\mid \mathrm{n}>$ é autoestado de $\hat{\mathrm{C}}_{\mathrm{ij}}$ : 


$$
\begin{aligned}
& \mathrm{C}_{11}\left|\mathrm{n}>=\left(2 \mathrm{n}+\mathrm{n}_{01}\right)\right| \mathrm{n}> \\
& \mathrm{C}_{22}\left|\mathrm{n}>=\mathrm{n}_{02}\right| \mathrm{n}> \\
& \mathrm{C}_{33}\left|\mathrm{n}>=\mathrm{n}_{03}\right| \mathrm{n}>
\end{aligned}
$$

e é aniquilado pelos abaixadores de $\mathrm{SU}(3)$ :

$$
\mathrm{C}_{\mathrm{ij}} \mid \mathrm{n}>=0, \quad \mathrm{i}<\mathrm{j}
$$

onde $\mathrm{C}_{\mathrm{ii}}\left|0>=\mathrm{n}_{\mathrm{oi}}\right| 0>$.

Portanto segue que os estados $\mid \mathrm{n}>$ são estados de peso mínimo de $\mathrm{SU}(3)$ com rótulos de Elliott $(\lambda+2 \mathrm{n} \mu)$ onde $\left(\lambda=\mathrm{n}_{01}-\mathrm{n}_{02} \quad \mu=\mathrm{n}_{02}-\mathrm{n}_{03}\right)$ são os rótulos associados ao estado $|0\rangle$. Os rótulos $(\lambda \mu)$ foram definidos no capítulo anterior.

O subespaço coletivo gerado pelo modelo $\mathrm{Sp}_{11}(1, \mathrm{R})$, é obtido pela projeção em momento angular dos estados (7), isto é:

$$
\left.\left|\mathrm{n}, \mathrm{LMK}>=\mathrm{P}_{\mathrm{MK}}^{\mathrm{L}}\right| \mathrm{n}\right\rangle \text {. }
$$

Os estados acima são autoestados da hamiltoniana do oscilador harmônico esférico, dada em termos de coordenadas intrínsecas, com energia de excitação $2 n \hbar \omega$ e portanto são ortogonais no rótulo $n$. No entanto esses estados não são ortogonais no rótulo K .

Para construírmos uma base ortogonal e completa a partir dos estados (8) vamos nos basear nos resultados obtidos nas referências 2 e 16 . O procedimento consiste em primeiro projetar em momento angular os estados (8), de acordo com a seguinte regra, obtida por Elliott: 
Os valores para o rótulo $\mathrm{K}$ são dados por:

$$
\mathrm{K}=\min (\lambda \mu), \min (\lambda \mu)-2, \ldots, 1,0
$$

e os correspondentes valores do momento angular orbital L são dados por:

$$
\begin{array}{ll}
\mathrm{L}=\mathrm{K}, \mathrm{K}+1, \ldots, \mathrm{K}+\operatorname{máx}(\lambda \mu) & (\mathrm{K} \neq 0) \\
\mathrm{L}=\operatorname{máx}(\lambda \mu), \text { máx }(\lambda \mu)-2, \ldots, 1,0 \quad(\mathrm{~K}=0)
\end{array}
$$

onde $(\lambda \mu)$ são os rótulos de Elliott do estado (8).

Por fim utilizamos o procedimento utilizado por $\operatorname{Vergados}^{(16)}$, que consiste na ortogonalização pelo processo de Gram-Schmidt dos estados projetados de acordo com a regra de Elliott. No caso particular em que consideramos estados de peso mínimo $|0\rangle$, axialmente simétricos e portanto com rótulos $(\lambda 0)$ de $\mathrm{SU}(3)$, somente projetamos estados com $\mathrm{K}=0$ e os possíveis valores de $\mathrm{L}$, obtidos em (8) são restritos pela relação $\mathrm{L} \leq \lambda+2 \mathrm{n}$.

\subsection{Determinação do Subespaço Coletivo}

Como discutimos na introdução desta tese, vamos procurar analisar as propriedades do modelo simplético em uma base deformada, através do cálculo no submodelo $\mathrm{Sp}(1, \mathrm{R})$, envolvendo estados de peso mínimo prolato, oblato e triaxial. O nosso objetivo nesta seção é analisar a estrutura dos estados do subespaço coletivo gerados pelos três modelos 
$\mathrm{Sp}(1, \mathrm{R})$ acima, em base esférica e que são em princípio associados à níveis de baixa energia do ${ }^{12} \mathrm{C}$.

Para analisarmos a base oblata, vamos realizar um cálculo do tipo $\mathrm{Sp}_{\perp}(1, \mathrm{R})$ onde o estado de peso mínimo é dado pelo determinante de Slater oblato, obtido na eq.(3.5) do capítulo anterior e que denominaremos estado 0 -partícula 0 -buraco na nomenclatura do modelo de camadas do oscilador. Os estados desse modelo são associados à banda rotacional fundamental do ${ }^{12} \mathrm{C}$.

A base de estados prolata é obtida pelo cálculo no modelo $\mathrm{Sp}_{\| 1}(1, \mathrm{R})$, associado ao estado de peso mínimo dado pelo determinante de Slater prolato, obtido na eq.(3.20) que denominaremos estado 4-partículas 4-buracos na nomenclatura do modelo de camadas do oscilador. Este modelo está associado ao segundo estado $0_{2}^{+}$, observado a uma energia de excitação de $7.65 \mathrm{MeV}{ }^{(12)}$, e que pode ser considerado como um estado intruso que vem de uma configuração 4-partículas 4-buracos. $(10),(15)$

Os estados de base triaxiais serão analisados através de um cálculo do tipo $\mathrm{Sp}_{\mathrm{N}}(1, \mathrm{R})$, onde o estado de peso mínimo triaxial é dado pela eq.(3.19), e denominaremos tal estado de 2-partículas 2-buracos na nomenclatura do modelo de camadas do oscilador.

\subsubsection{Modelo $\mathrm{Sp}_{\perp}(1, \mathrm{R})$ associado ao estado de peso mínimo op-ob}

Vamos agora considerar o submodelo $\mathrm{Sp}_{\perp}(1, \mathrm{R})$ associado ao estado de peso mínimo, 3.5, que reescreveremos abaixo usando uma forma que vai ser conveniente para os desenvolvimento posteriores: 


$$
\begin{aligned}
\mid 0(04) ; \mathrm{b}_{0}>^{\mathrm{e}}= & \frac{1}{\sqrt{12 !}} \operatorname{det}(000)^{\mathrm{n}+}(010)^{\mathrm{n}_{+}}(001)^{\mathrm{n}+}(000)^{\mathrm{n}-}(010)^{\mathrm{n}-}(001)^{\mathrm{n}-} \\
& \cdot(000)^{\mathrm{p}+}(010)^{\mathrm{p}_{+}}(001)^{\mathrm{p}+}(000)^{\mathrm{p}-}(010)^{\mathrm{p}-}(001)^{\mathrm{p}-}
\end{aligned}
$$

Alteramos a notação utilizada no capítulo-3, omitindo os rótulos relativos à simetria permutacional orbital e ao spin e isospin. Inclúmos o comprimento do oscilador $b_{0}$, afim de indicar que a função de onda, (9) depende explicitamente de $b_{0}$.

Mostramos no capítulo 2, seção 2.4 , que os operadores da álgebra $\operatorname{sp}(3, \mathrm{R})$ se decompõem em componentes intrínsecas e do centro de massa, portanto, podemos escrever:

$$
\begin{aligned}
& \mathrm{A}_{\perp}^{+\mathrm{e}}\left(\mathrm{b}_{0}\right)=\mathrm{A}_{\perp}^{+}\left(\mathrm{b}_{0}\right)+\mathrm{A}_{\perp}^{+} \mathrm{CM} \\
& \mathrm{A}_{\perp}^{\mathrm{e}}\left(\mathrm{b}_{0}\right)=\mathrm{A}_{\perp}\left(\mathrm{b}_{0}\right)+\mathrm{A}_{\perp}^{\mathrm{CM}}
\end{aligned}
$$

onde

$$
\begin{aligned}
& \mathrm{A}_{\perp}^{+\mathrm{e}}\left(\mathrm{b}_{0}\right)=\mathrm{A}_{22}^{+\mathrm{e}}\left(\mathrm{b}_{0}\right)+\mathrm{A}_{33}^{+\mathrm{e}}\left(\mathrm{b}_{0}\right) \\
& \mathrm{A}_{\perp}^{+}\left(\mathrm{b}_{0}\right)=\mathrm{A}_{22}^{+}\left(\mathrm{b}_{0}\right)+\mathrm{A}_{33}^{+}\left(\mathrm{b}_{0}\right)
\end{aligned}
$$

$\mathrm{e}$

$$
\mathrm{A}_{\perp}^{+} \mathrm{CM}=\mathrm{A}_{22}^{+} \mathrm{CM}+\mathrm{A}_{33}^{+} \mathrm{CM}=\frac{1}{2 \mathrm{~A}} \sum_{i=1}^{\mathrm{A}} \mathrm{a}_{2 ; \mathrm{i}}^{+} \mathrm{a}_{2 ; \mathrm{i}}^{+}+\frac{1}{2 \mathrm{~A}} \sum_{i=1}^{\mathrm{A}} \mathrm{a}_{3 ; \mathrm{i}}^{+} \mathrm{a}_{3 ; \mathrm{i}}^{+}
$$

e também

$$
\mathrm{A}_{\perp}^{\mathrm{e}}\left(\mathrm{b}_{0}\right)=\left[\mathrm{A}_{\perp}^{+\mathrm{e}}\left(\mathrm{b}_{0}\right)\right]^{+}, \quad \mathrm{A}_{\perp}\left(\mathrm{b}_{0}\right)=\left[\mathrm{A}_{\perp}^{+}\left(\mathrm{b}_{0}\right)\right]^{+}, \mathrm{A}_{\perp}^{\mathrm{CM}}=\left[\mathrm{A}_{\perp}^{+} \mathrm{CM}\right]^{+} .
$$

A partir da definição de $\mathrm{a}_{\mu}^{\mathrm{CM}}$, dada na seção 2.4, é fácil mostrar que o estado (9) satisfaz a 
seguinte relação:

$$
\mathrm{a}_{\mu}^{\mathrm{CM}} \mid 0(04) ; \mathrm{b}_{0}>^{\mathrm{e}}=0
$$

e portanto o estado acima pode ser fatorado da seguinte maneira:

$$
\left|0(04) ; b_{0}>^{e}=\right| 0(04) ; b_{0}>\mid 0>^{C M}
$$

onde $\left|0(04) ; b_{0}\right\rangle$ é dado em termos de coordenadas intrínsecas e $|0\rangle^{\mathrm{CM}}$ corresponde ao estado fundamental do oscilador do centro de massa.

Partindo da relação (10) e da decomposição em componentes intrínseca e de centro de massa da álgebra $\mathrm{sp}_{\perp}(1, \mathrm{R})$, é fácil mostrar que a ação dos operadores definidos em (2) é dada por:

$$
\mathrm{A}_{0_{\perp}}\left(\mathrm{b}_{0}\right)\left|0(04) ; \mathrm{b}_{0}>=\mathrm{k}_{\perp}\right| 0(04) ; \mathrm{b}_{0}>\operatorname{com} \mathrm{k}_{\perp}=19 / 2
$$

e

$$
\mathrm{A}_{\perp}\left(\mathrm{b}_{0}\right) \mid 0(04) ; \mathrm{b}_{0}>=0
$$

Portanto, o estado acima é estado de peso mínimo de $\mathrm{Sp}_{\perp}(1, \mathrm{R})$, com rótulo $\mathrm{k}_{\perp}=19 / 2$. Analogamente de (5), temos que

$$
\mathrm{A}_{011}\left(\mathrm{~b}_{0}\right)\left|0(04) ; \mathrm{b}_{0}>=\mathrm{k}_{11}\right| 0(04) ; \mathrm{b}_{0}>\text { com } \mathrm{k}_{11}=11 / 4
$$

e

$$
\mathrm{A}_{11}\left(\mathrm{~b}_{0}\right) \mid 0(04) ; \mathrm{b}_{0}>=0
$$

De acordo com as expressões 3 e 4 temos que a base de estados do subespaço 
coletivo é gerado pelos seguintes estados ortogonais:

$$
\left|\mathrm{n}(04), \mathrm{LM} 0 ; \mathrm{b}_{0}>=\mathrm{P}_{\mathrm{M} 0}^{\mathrm{L}}\right| \mathrm{n}(04) ; \mathrm{b}_{0}>\quad, \quad \mathrm{n}=0,1, \ldots
$$

onde

$$
\left|\mathrm{n}(04) ; \mathrm{b}_{0}>=\left[\mathrm{A}_{\perp}^{+}\left(\mathrm{b}_{0}\right)\right]^{\mathrm{n}}\right| 0(04) ; \mathrm{b}_{0}>\left[\frac{\Gamma(19)}{\Gamma(\mathrm{n}+1) \Gamma(\mathrm{n}+19)}\right]^{1 / 2} .
$$

Como vimos na seção 4.2.1, os valores de L obtidos pela projeção em momento angular dos estados acima, são sempre pares e satisfazem a relação $L \leq 4+2 n$.

\subsubsection{Modelo $\mathrm{Sp}_{\| 1}(1, \mathrm{R})$ Associado ao Estado de Peso Mínimo $2 \mathrm{p}-2 \mathrm{~b}$}

Neste caso o estado de peso mínimo é dado pela expressão (3.19), que reescrevemos abaixo numa forma mais conveniente para os desenvolvimentos futuros:

$$
\left|0(62) ; \mathrm{b}_{0}\right\rangle^{\mathrm{e}}=\frac{1}{\sqrt{6}} \sum_{\{\mathrm{C}\}}\left|\psi^{\{\mathrm{C}\}} ; \mathrm{b}_{0}\right\rangle^{\mathrm{e}}
$$

A expressão acima é dada pela soma de seis determinantes de Slater, $\left|\psi^{\{\mathrm{C}\}} ; \mathrm{b}_{0}\right\rangle^{\mathrm{e}}$, determinado pelo rótulo $\{\mathrm{C}\}$, o qual pode assumir um dos seis conjuntos de números seguintes:

$$
\{C\} \in\{(1122),(2211),(1212),(1221),(2112)(2121)\}
$$

Para obtermos o estado $\left|\psi^{\{\mathrm{C}\}} ; \mathrm{b}_{0}\right\rangle^{\mathrm{e}}$ implícito na notação acima, associamos o número 1 
aos estados $(000)(100)(010)$ e o número 2 aos estados $(000)(100)(200)$ e assim o rótulo $\{\mathrm{C}\}=\left(\mathrm{m}_{1} \mathrm{~m}_{2} \mathrm{~m}_{3} \mathrm{~m}_{4}\right) \quad$ dado acima indica que, por exemplo, no determinante $\left|\psi^{(1122)} ; \mathrm{b}_{0}\right\rangle^{\mathrm{e}}$ colocamos um neutron com spin para cima e um neutron com spin para baixo nos estados 1 e um proton com spin para cima e um proton com spin para baixo no estados 2, isto é:

$$
\begin{aligned}
\left|\psi^{(1122)} ; \mathrm{b}_{0}\right\rangle^{\mathrm{e}}= & \frac{1}{\sqrt{12 !}} \operatorname{det}(000)^{\mathrm{n}+}(100)^{\mathrm{n}+}(010)^{\mathrm{n}+}(000)^{\mathrm{n}-}(100)^{\mathrm{n}-}(010)^{\mathrm{n}-} \\
& \cdot(000)^{\mathrm{p}+}(100)^{\mathrm{p}+}(200)^{\mathrm{p}+}(000)^{\mathrm{p}-}(100)^{\mathrm{p}-}(200)^{\mathrm{p}-}
\end{aligned}
$$

onde omitimos na notação os mesmos rótulos como fizemos na seção anterior.

Analogamente à seção anterior, $\mathrm{a}_{\mu}^{\mathrm{CM}} \mid 0(62) ; \mathrm{b}_{0}>^{\mathrm{e}}=0$ e portanto o estado (13) pode ser fatorado da seguinte maneira:

$$
\left|0(62) ; \mathrm{b}_{0}>^{\mathrm{e}}=\right| 0(62) ; \mathrm{b}_{0}>|0\rangle^{\mathrm{CM}}
$$

onde $\left|0(62) ; \mathrm{b}_{0}\right\rangle$ é dado em termos de coordenadas intrínsecas e $|0\rangle^{\mathrm{CM}}$ corresponde ao estado fundamental do oscilador do centro de massa.

A partir da seção 2.4 do capítulo 2 é imediato que:

$$
\begin{aligned}
& A_{11}^{+e}\left(b_{0}\right)=A_{11}^{+}\left(b_{0}\right)+A_{11}^{+} C M \\
& A_{0 " 1}^{e}\left(b_{0}\right)=A_{011}\left(b_{0}\right)+A_{0 "}^{C M} .
\end{aligned}
$$

A partir da decomposição acima e da fatoração dada pela expressão (14), é imediato 
que a ação dos operadores indicadas em (6) no estado $\left|0(62) ; \mathrm{b}_{0}\right\rangle$ é dada por:

$$
\begin{aligned}
& \mathrm{A}_{0^{\prime \prime}}\left(\mathrm{b}_{0}\right)\left|0(62) ; \mathrm{b}_{0}>=\mathrm{k}_{11}\right| 0(62) ; \mathrm{b}_{0}>\text { com } \mathrm{k}_{11}=27 / 4 \\
& \mathrm{~A}_{11}\left(\mathrm{~b}_{0}\right) \mid 0(62) ; \mathrm{b}_{0}>=0
\end{aligned}
$$

e portanto o estado $\left|0(62) ; \mathrm{b}_{0}\right\rangle$ é estado de peso mínimo de $\mathrm{Sp}_{11}(1, \mathrm{R})$ com rótulo $k_{11}=27 / 4$.

Analogamente podemos mostrar que

$$
\begin{aligned}
& \mathrm{A}_{0_{\perp}}\left(\mathrm{b}_{0}\right)\left|0(62) ; \mathrm{b}_{0}\right\rangle=\mathrm{k}_{\perp}\left|0(62) ; \mathrm{b}_{0}\right\rangle \text { com } \mathrm{k}_{\perp}=13 / 2 \\
& \mathrm{~A}_{\perp}\left(\mathrm{b}_{0}\right)\left|0(62) ; \mathrm{b}_{0}\right\rangle=0
\end{aligned}
$$

O subespaço coletivo gerado pelo modelo $\mathrm{Sp}_{\text {II }}(1, \mathrm{R})$ é obtido a partir das expressões (7) e (8):

$$
\left|\mathrm{n}(62), \mathrm{LMK} ; \mathrm{b}_{0}\right\rangle=\mathrm{P}_{\mathrm{MK}}^{\mathrm{L}}\left|\mathrm{n}(62) ; \mathrm{b}_{0}\right\rangle, \quad \mathrm{n}=0,1, \ldots
$$

com

$$
\left|\mathrm{n}(62) ; \mathrm{b}_{0}>=\left[\mathrm{A}_{11}^{+}\left(\mathrm{b}_{0}\right)\right]^{\mathrm{n}}\right| 0(62) ; \mathrm{b}_{0}>\left[\frac{\Gamma(27 / 2)}{\Gamma(\mathrm{n}+1) \Gamma(\mathrm{n}+27 / 2)}\right]^{1 / 2} .
$$

De acordo com a análise que fizemos na seção 4.2.2, temos que o estado $\mid \mathrm{n}(62) ; \mathrm{b}_{0}>$ é estado de peso mínimo da representação irredutível de $\mathrm{SU}(3)$ com rótulos de Elliott

$$
(\lambda \mu)=(6+2 \mathrm{n} 2)
$$


Usando a regra de Elliott, que discutimos na seção 4.2.2, segue que o estado, $\mid \mathrm{n}(62), \mathrm{LMK} ; \mathrm{b}_{0}>, \mathrm{n}=0,1, \ldots$, tem os seguintes valores para o rótulo $\mathrm{K}$ :

$$
\mathrm{K}=0,2
$$

e em correspondência os seguintes valores para o momento angular orbital L:

$$
\begin{array}{ll}
\mathrm{L}=2,3, \ldots, 8+2 \mathrm{n} & (\mathrm{K}=2) \\
\mathrm{L}=6+2 \mathrm{n}, 4+2 \mathrm{n}, \ldots, 0 & (\mathrm{~K}=0) .
\end{array}
$$

Os estados $\left|\mathrm{n}(62), \mathrm{LMK} ; \mathrm{b}_{0}\right\rangle$ geram uma base não ortogonal no rótulo $\mathrm{K}$, que ortogonalizamos pelo processo de Gram-Schmidt como discutimos na seção 4.2.2.

\subsubsection{Modelo $\mathrm{Sp}_{\| 1}(1, \mathrm{R})$ Associado ao Estado de Peso Mínimo $4 \mathrm{p}-4 \mathrm{~b}$}

O estado de peso mínimo, neste caso, é dado pela expressão 3.20 , que reescrevemos abaixo:

$$
\begin{aligned}
\mid 0(120) ; \mathrm{b}_{0}>^{\mathrm{e}} & =\frac{1}{\sqrt{12 !}} \operatorname{det}(000)^{\mathrm{n}+}(100)^{\mathrm{n}+}(200)^{\mathrm{n}+}(000)^{\mathrm{n}-}(100)^{\mathrm{n}-}(200)^{\mathrm{n}-} \\
& \cdot(000)^{\mathrm{P}+}(100)^{\mathrm{P}+}(200)^{\mathrm{p}+}(000)^{\mathrm{p}-}(100)^{\mathrm{p}-}(200)^{\mathrm{p}-}
\end{aligned}
$$

A notação é a mesma da seção 4.3.1. 
Analogamente às seções 4.3.1, 4.3.2, $\mathrm{a}_{\mu}^{\mathrm{CM}} \mid 0(120) ; \mathrm{b}_{0}>^{\mathrm{e}}=0$ e portanto temos a fatoração seguinte:

$$
\left|0(120) ; \mathrm{b}_{0}>^{\mathrm{e}}=\right| 0(120) ; \mathrm{b}_{0}>\mid 0>^{\mathrm{CM}}
$$

onde $\mid 0(120) ; \mathrm{b}_{0}>$ é dado em termos de coordenadas intrínsecas e $\mid 0>^{\mathrm{CM}}$ corresponde ao estado fundamental do oscilador do centro de massa.

A partir das relações 15 e da fatoração 19, segue imediatamente que a ação dos operadores que determinam o estado de peso mínimo do grupo $\operatorname{Sp}_{\|}(1, \mathrm{R})$, definido pelas expressões 6, é dada por:

$$
\begin{aligned}
& \mathrm{A}_{011}\left(\mathrm{~b}_{0}\right)\left|0(120) ; \mathrm{b}_{0}>=\mathrm{k}_{11}\right| 0(120) ; \mathrm{b}_{0}>\text { com } \mathrm{k}_{11}=35 / 4 \\
& \mathrm{~A}_{11}\left(\mathrm{~b}_{0}\right) \mid 0(120) ; \mathrm{b}_{0}>=0
\end{aligned}
$$

o que mostra que $\mid 0(120) ; b_{0}>$ é estado de peso mínimo de $\operatorname{Sp}_{11}(1, \mathrm{R})$ com rótulo $\mathrm{k}_{11}=35 / 4$.

Analogamente podemos mostrar que

$$
\mathrm{A}_{0_{\perp}}\left(\mathrm{b}_{0}\right)\left|0(120) ; \mathrm{b}_{0}>=\mathrm{k}_{\perp}\right| 0(120) ; \mathrm{b}_{0}>\quad \mathrm{com} \quad \mathrm{k}_{\perp}=11 / 2 \text { e } \mathrm{A}_{\perp}\left(\mathrm{b}_{0}\right) \mid 0(120) ; \mathrm{b}_{0}>=0 \quad
$$

A partir das expressões (7), (8) obtemos a base do sub-espaço coletivo gerado pelo submodelo $\mathrm{Sp}_{\| 1}(1, \mathrm{R})$ neste caso, isto é:

$$
\left|\mathrm{n}(120), \mathrm{LM} 0 ; \mathrm{b}_{0}\right\rangle=\mathrm{P}_{\mathrm{M} 0}^{\mathrm{L}}\left|\mathrm{n}(120) ; \mathrm{b}_{0}\right\rangle \quad, \quad \mathrm{n}=0,1, \ldots
$$


com

$$
\left|\mathrm{n}(120) ; \mathrm{b}_{0}>=\left[\mathrm{A}_{11}^{+}\left(\mathrm{b}_{0}\right)\right]^{\mathrm{n}}\right| 0(120) ; \mathrm{b}_{0}>\left[\frac{\Gamma(35 / 2)}{\Gamma(\mathrm{n}+1) \Gamma(\mathrm{n}+35 / 2)}\right]^{1 / 2} .
$$

Como mostramos na seção 4.2.2, na expressão acima L é par e satisfaz a relação $\mathrm{L} \leq 12+2 \mathrm{n}$.

\subsection{Cálculo Analítico dos Elementos de Matriz dos Operadores de Interesse}

O subespaço coletivo no qual vamos diagonalizar a hamiltoniana e analisar as propriedades espectroscópicas é gerado pelos estados (12), (17) e (21) obtidos acima. Vamos a seguir considerar o cálculo dos elementos de matriz dos observáveis nestes estados, utilizando o MFG especificamente para o cálculo do operador energia potencial, que não pode ser escrito em termos dos geradores da álgebra $\mathrm{sp}(3, \mathrm{R})$ e para o cálculo do overlap. Uma vez que a energia cinética e os operadores de monopolo e quadrupolo podem ser escritos diretamente em função dos geradores da álgebra $\mathrm{sp}(3, \mathrm{R})$, estes operadores serão calculados diretamente por meio de procedimentos algébricos.

\subsubsection{Método da Função Geratriz}

O objetivo desta seção é obter um método que permita o cálculo de um operador arbitrário $\hat{\mathrm{O}}$ nos estados de base dos submodelos $\mathrm{Sp}_{\perp}(1, \mathrm{R}), \mathrm{Sp}_{11}(1, \mathrm{R})$. Como veremos, todos os desenvolvimentos que iremos fazer, vai depender exclusivamente da álgebra $\operatorname{sp}(1, R)$ e portanto vamos tratar de uma maneira unificada os casos $\operatorname{Sp}_{\|}(1, \mathrm{R})$ e $\mathrm{Sp}_{\perp}(1, \mathrm{R})$. Vamos definir genericamente a álgebra $\mathrm{sp}(1, \mathrm{R})$ por: 
$\mathrm{A}, \mathrm{A}^{+}, \mathrm{A}_{0}$

e o seu estado de peso mínimo por:

$$
\begin{aligned}
& \mathrm{A}_{0}\left|0 ; \mathrm{b}_{0}>=\mathrm{k}\right| 0 ; \mathrm{b}_{0}> \\
& \mathrm{A} \mid 0 ; \mathrm{b}_{0}>=0
\end{aligned}
$$

os estados de base do modelo $\mathrm{Sp}(1, \mathrm{R})$ são dados por:

$$
\left.\left|\mathrm{n}, \mathrm{LMK} ; \mathrm{b}_{0}>=\mathrm{P}_{\mathrm{MK}}^{\mathrm{L}}\right| \mathrm{n} ; \mathrm{b}_{0}\right\rangle
$$

onde

$$
\left|\mathrm{n} ; \mathrm{b}_{0}\right\rangle=\left(\mathrm{A}^{+}\right)^{\mathrm{n}} \mid 0 ; \mathrm{b}_{0}>\cdot\left[\frac{\Gamma(2 \mathrm{k})}{\Gamma(\mathrm{n}+1) \Gamma(\mathrm{n}+2 \mathrm{k})}\right]^{1 / 2} .
$$

A álgebra $\mathrm{sp}(1, \mathrm{R})$, definida pelos operadores $(22)$, corresponde à álgebra $\mathrm{sp}_{\perp}(1, \mathrm{R})$ ou $\mathrm{sp}_{\| 1}(1, \mathrm{R})$ definida pelos operadores (1) e (5) respectivamente. O operador projeção em momento angular é definido por:

$$
\mathrm{P}_{\mathrm{MK}}^{\mathrm{L}}=\frac{(2 \mathrm{~L}+1)}{8 \pi^{2}} \int \mathrm{d} \Omega \mathrm{D}_{\mathrm{MK}}^{\mathrm{L}}(\Omega)^{*} \mathrm{R}(\Omega)
$$

onde 


$$
\begin{aligned}
& \int \mathrm{d} \Omega=\int_{0}^{2 \pi} \mathrm{d} \alpha \int_{0}^{\pi} \mathrm{d} \beta \operatorname{sen} \beta \int_{0}^{2 \pi} \mathrm{d} \gamma \\
& \mathrm{R}(\Omega)=\mathrm{e}^{-\mathrm{i} \alpha \mathrm{L}_{\mathrm{z}}} \mathrm{e}^{-\mathrm{i} \beta \mathrm{L}_{\mathrm{y}}} \mathrm{e}^{-\mathrm{i} \gamma \mathrm{L}_{\mathrm{z}}}(\hbar=1) \\
& \mathrm{D}_{\mathrm{MK}}^{\mathrm{L}(\Omega)}=<\mathrm{LM}|\mathrm{R}(\Omega)| \mathrm{LK}>=\mathrm{e}^{-\mathrm{i} \alpha \mathrm{M}}<\mathrm{LM}\left|\mathrm{e}^{-\mathrm{i} \beta \mathrm{L}_{\mathrm{y}}}\right| \mathrm{LK}>\mathrm{e}^{-\mathrm{i} \gamma \mathrm{K}}= \\
& =\mathrm{e}^{-\mathrm{i} \alpha \mathrm{M}} \mathrm{d}_{\mathrm{MK}}^{\mathrm{L}}(\beta) \mathrm{e}^{-\mathrm{i} \gamma \mathrm{K}}
\end{aligned}
$$

onde $\mathrm{d}_{\mathrm{MK}}^{\mathrm{L}}(\beta)$ é denominada matriz de rotação reduzida.

Vamos definir:

$$
\left|\theta ; \mathrm{b}_{0}\right\rangle=\mathrm{e}^{-\mathrm{i} \theta \mathrm{S}}\left|0 ; \mathrm{b}_{0}\right\rangle
$$

onde $\mathrm{S}=\mathrm{i}\left(\mathrm{A}^{+}-\mathrm{A}\right)$ corresponde ao operador gerador de transformação de escala. De uma maneira mais explícita, $\mathrm{S}$ corresponde a

$$
\begin{aligned}
& S_{11}=i\left[A_{11}^{+}-A_{11}\right] \\
& S_{\perp}=i\left[A_{\perp}^{+}-A_{\perp}\right] .
\end{aligned}
$$

No capítulo 5 estudaremos com detalhes a ação dos operadores acima. Abaixo escrevemos a fórmula $\mathrm{BCH}$ do grupo $\mathrm{Sp}(1, \mathrm{R})$ que pode ser obtida facilmente pela técnica discutida no apêndice 2. 


$$
\mathrm{e}^{-\mathrm{i} \theta \mathrm{S}}=\mathrm{e}^{\theta\left(\mathrm{A}^{+}-\mathrm{A}\right)}=\mathrm{e}^{\operatorname{tagh} \theta \mathrm{A}^{+}} \mathrm{e}^{\ln \left(1-\operatorname{tagh}^{2} \theta\right) \mathrm{A}_{0}} \mathrm{e}^{-\operatorname{tagh} \theta \mathrm{A}} .
$$

Substituindo a expressão acima em (26), levando em conta que $\left|0 ; \mathrm{b}_{0}\right\rangle$ é estado de peso mínimo da álgebra $\operatorname{sp}(1, \mathrm{R})$ e definindo $\tilde{\beta}=\operatorname{tagh} \theta$ obtemos:

$$
\left|\theta ; \mathrm{b}_{0}>=\left(1-\tilde{\beta}^{2}\right)^{\mathrm{k}} \mathrm{e}^{\tilde{\beta} \mathrm{A}^{+}}\right| 0 ; \mathrm{b}_{0}>
$$

Segue imediatamente da expressão acima que

$$
\left(\mathrm{A}^{+}\right)^{\mathrm{n}}\left|0 ; \mathrm{b}_{0}>=\frac{\partial^{\mathrm{n}}}{\partial \tilde{\beta}^{\mathrm{n}}} \frac{\mid \theta ; \mathrm{b}_{0}>}{\left(1-\tilde{\beta}^{2}\right)^{\mathrm{k}}}\right|_{\tilde{\beta}=0}=\frac{\partial^{\mathrm{n}}}{\partial \tilde{\beta}^{\mathrm{n}}} \mathrm{e}^{\tilde{\beta} \mathrm{A}^{+}}\left|0 ; \mathrm{b}_{0}>\right|_{\tilde{\beta}=0}
$$

Substituindo a expressão acima em (24) obtemos

$$
\begin{aligned}
& \left|\mathrm{n} ; \mathrm{b}_{0}>=\frac{\Gamma(2 \mathrm{k})^{1 / 2}}{(\Gamma(\mathrm{n}+1) \Gamma(\mathrm{n}+2 \mathrm{k}))^{1 / 2}} \frac{\partial^{\mathrm{n}}}{\partial \tilde{\beta}^{\mathrm{n}}} \frac{\mid \theta ; \mathrm{b}_{0}>}{\left(1-\tilde{\beta}^{2}\right)^{\mathrm{k}}}\right|_{\tilde{\beta}=0}= \\
& \frac{\Gamma(2 \mathrm{k})^{1 / 2}}{(\Gamma(\mathrm{n}+1) \Gamma(\mathrm{n}+2 \mathrm{k}))^{1 / 2}} \frac{\partial^{\mathrm{n}}}{\partial \tilde{\beta}^{\mathrm{n}}} \mathrm{e}^{\tilde{\beta} \mathrm{A}^{+}}\left|0 ; \mathrm{b}_{0}>\right|_{\tilde{\beta}=0} .
\end{aligned}
$$

Se Ô é um operador arbitrário, a partir das expressões (24) e (29) e da propriedade do projetor $\left[\mathrm{P}_{\mathrm{MK}}^{\mathrm{L}}\right]^{+}=\mathrm{P}_{\mathrm{KM}}^{\mathrm{L}}$ podemos escrever: 


$$
\begin{aligned}
& \left.<\mathrm{n}^{\prime}, \mathrm{L}^{\prime} \mathrm{M}^{\prime} \mathrm{K}^{\prime} ; \mathrm{b}_{0}|\hat{\mathrm{O}}| \mathrm{n}, \mathrm{LMK} ; \mathrm{b}_{0}\right\rangle=\left\langle\mathrm{n}^{\prime} ; \mathrm{b}_{0}\left|\mathrm{P}_{\mathrm{K}^{\prime} \mathrm{M}^{\prime}}^{\mathrm{L}^{\prime}} \hat{\mathrm{O}} \mathrm{P}_{\mathrm{MK}}^{\mathrm{L}}\right| \mathrm{n} ; \mathrm{b}_{0}\right\rangle= \\
& =\frac{\Gamma(2 \mathrm{k})}{\left(\Gamma\left(\mathrm{n}^{\prime}+1\right) \Gamma(\mathrm{n}+1) \Gamma\left(\mathrm{n}^{\prime}+2 \mathrm{k}\right) \Gamma(\mathrm{n}+2 \mathrm{k})\right)^{1 / 2}} \frac{\partial^{\mathrm{n}^{\prime}}}{\partial \tilde{\beta}^{\mathrm{n}^{\prime}}} \frac{\partial^{\mathrm{n}}}{\partial \tilde{\beta}^{\mathrm{n}}} \cdot \\
& \cdot<0 ; \mathrm{b}_{0}\left|\mathrm{e}^{\tilde{\beta}^{\prime} \mathrm{A}} \mathrm{P}_{\mathrm{K}^{\prime} \mathrm{M}^{\prime}}^{\mathrm{L}^{\prime}} \hat{\mathrm{O}} \mathrm{P}_{\mathrm{MK}}^{\mathrm{L}} \mathrm{e}^{\tilde{\beta} \mathrm{A}^{+}}\right| 0 ; \mathrm{b}_{0}>\mid \begin{array}{l}
\tilde{\beta}_{\tilde{\beta}}=0 \\
\tilde{\beta}^{\prime}=0
\end{array}
\end{aligned}
$$

A expressão acima determina o que chamamos de método da função geratriz, que permite o cálculo dos elementos de matriz de um operador Ô na base (24), em função de derivadas da expressão:

$$
<0 ; \mathrm{b}_{0}\left|\mathrm{e}^{\tilde{\beta}^{\prime} \mathrm{A}} \mathrm{P}_{\mathrm{K}^{\prime} \mathrm{M}^{\prime}}^{\mathrm{L}^{\prime}} \hat{\mathrm{O}} \mathrm{P}_{\mathrm{MK}}^{\mathrm{L}} \mathrm{e}^{\tilde{\beta} \mathrm{A}^{+}}\right| 0 ; \mathrm{b}_{0}>
$$

que chamamos de função geratriz.

\section{Operador Escalar}

Como os operadores que serão calculados pelo MFG são escalares, vamos analisar este caso em particular. Se Ô é um operador escalar por rotação é imediato que ele comuta com $\mathrm{P}_{\mathrm{MK}}^{\mathrm{L}}$ e como temos a seguinte propriedade do projetor:

$$
\mathrm{P}_{\mathrm{K}^{\prime} \mathrm{M}^{\prime}}^{\mathrm{L}^{\prime}} \mathrm{P}_{\mathrm{MK}}^{\mathrm{L}}=\delta_{\mathrm{LL}^{\prime}} \delta_{\mathrm{MM}^{\prime}}, \mathrm{P}_{\mathrm{K}^{\prime} \mathrm{K}}^{\mathrm{L}}
$$


podemos reescrever o elemento de matriz 4.30 como:

$$
\begin{aligned}
& <\mathrm{n}^{\prime}, \mathrm{L}^{\prime} \mathrm{M}^{\prime} \mathrm{K}^{\prime} ; \mathrm{b}_{0}|\hat{\mathrm{O}}| \mathrm{n}, \mathrm{LMK} ; \mathrm{b}_{0}>=\frac{\Gamma(2 \mathrm{k}) \delta_{\mathrm{LL}^{\prime}} \delta_{\mathrm{MM}^{\prime}}}{\left(\Gamma\left(\mathrm{n}^{\prime}+1\right) \Gamma(\mathrm{n}+1) \Gamma\left(\mathrm{n}^{\prime}+2 \mathrm{k}\right) \Gamma(\mathrm{n}+2 \mathrm{k})\right)^{1 / 2}} . \\
& \cdot \frac{\partial^{\mathrm{n}^{\prime}}}{\partial \tilde{\beta}^{\mathrm{n}^{\prime}}} \frac{\partial^{\mathrm{n}}}{\partial \tilde{\beta}^{\mathrm{n}}}<0 ; \mathrm{b}_{0}\left|\mathrm{e}^{\tilde{\beta}^{\prime} \mathrm{A}} \hat{\mathrm{O}} \mathrm{P}_{\mathrm{K}^{\prime} \mathrm{K}}^{\mathrm{L}} \mathrm{e}^{\tilde{\beta} \mathrm{A}^{+}}\right| 0 ; \mathrm{b}_{0}>\mid \begin{array}{l}
\tilde{\beta}^{\prime}=0 \\
\tilde{\beta}=0
\end{array}
\end{aligned}
$$

onde

$$
\begin{aligned}
& <0 ; \mathrm{b}_{0}\left|\mathrm{e}^{\tilde{\beta}^{\prime} \mathrm{A}} \hat{\mathrm{O}} \mathrm{P}_{\mathrm{K}^{\prime} \mathrm{K}}^{\mathrm{L}} \mathrm{e}^{\tilde{\beta} \mathrm{A}^{+}}\right| 0 ; \mathrm{b}_{0}>=\frac{(2 \mathrm{~L}+1)}{8 \pi^{2}} \int \mathrm{d} \Omega \mathrm{D}_{\mathrm{K}^{\prime} \mathrm{K}}^{\mathrm{L}}(\Omega)^{*} . \\
& \cdot<0 ; \mathrm{b}_{0}\left|\mathrm{e}^{\tilde{\beta^{\prime} \mathrm{A}}} \hat{\mathrm{O}} \mathrm{R}(\Omega) \mathrm{e}^{\tilde{\beta} \mathrm{A}^{+}}\right| 0 ; \mathrm{b}_{0}>.
\end{aligned}
$$

No caso particular de $\mathrm{Sp}(1, \mathrm{R})$ axialmente simétricas, a expressão acima se reduz a:

$$
\begin{aligned}
& <0 ; \mathrm{b}_{0}\left|\mathrm{e}^{\tilde{\beta}^{\prime} \mathrm{A}} \hat{\mathrm{O}} \mathrm{P}_{00}^{\mathrm{L}} \mathrm{e}^{\tilde{\beta} \mathrm{A}^{+}}\right| 0 ; \mathrm{b}_{0}>=\frac{(2 \mathrm{~L}+1)}{2} \int_{0}^{\pi} \mathrm{d} \beta \sin \beta \mathrm{P}_{\mathrm{L}}(\cos \beta) . \\
& \cdot<0 ; \mathrm{b}_{0}\left|\mathrm{e}^{\tilde{\beta}^{\prime} \mathrm{A}} \hat{\mathrm{O}} \mathrm{R}_{\mathrm{y}}(\beta) \mathrm{e}^{\tilde{\beta} \mathrm{A}^{+}}\right| 0 ; \mathrm{b}_{0}>.
\end{aligned}
$$

As expressões (32), (33) e (34) serão usadas na próximas seções para o cálculo dos elementos de matriz do potencial e do overlap. 


\subsubsection{Cálculo do Overlap através do MFG}

Para obtermos os elementos de matriz do overlap através do MFG, precisamos realizar o cálculo indicado na expressão (32), no caso particular em que Ô = I, isto é, Ô é o operador escalar identidade. Como discutimos na seçào 4.3, em todos os casos que iremos considerar, o estado de peso mínimo $\left|0 ; \mathrm{b}_{0}\right\rangle^{\mathrm{e}}$, fatora-se da seguinte maneira:

$$
\left.\left|0 ; \mathrm{b}_{0}\right\rangle^{\mathrm{e}}=\left|0 ; \mathrm{b}_{0}>\right| 0\right\rangle^{\mathrm{CM}}
$$

e a álgebra $\operatorname{sp}(1, \mathrm{R})$ se decompõe da seguinte maneira:

$$
\begin{aligned}
& \mathrm{A}^{+\mathrm{e}}=\mathrm{A}^{+}+\mathrm{A}^{+} \mathrm{CM} \\
& \mathrm{A}^{\mathrm{e}}=\mathrm{A}+\mathrm{A}^{\mathrm{CM}} \\
& \mathrm{A}_{0}^{\mathrm{e}}=\mathrm{A}_{0}+\mathrm{A}_{0}^{\mathrm{CM}}
\end{aligned}
$$

onde recordamos que as relações acima se referem às álgebra $\mathrm{sp}_{11}(1, \mathrm{R})$ ou $\mathrm{sp}_{\perp}(1, \mathrm{R})$ de acordo com o caso que estivermos considerando.

Usando a fatoração (35) e a decomposição da álgebra (36) obtemos:

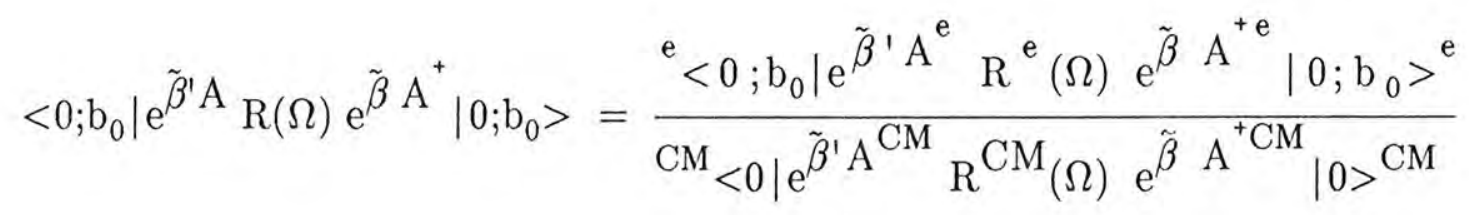


onde $\mathrm{R}^{\mathrm{e}}(\Omega)=\mathrm{R}(\Omega) \mathrm{R}^{\mathrm{CM}}(\Omega)$.

O estado $\left|0 ; \mathrm{b}_{0}\right\rangle^{\mathrm{e}}$, nos casos que iremos considerar, corresponde a um ou uma soma de determinantes de Slater, construído a partir de funções de onda do oscilador harmônico. Como $\mathrm{R}^{\mathrm{e}}(\Omega)$ e $\mathrm{e}^{\tilde{\beta} \mathrm{A}^{+\mathrm{e}}}$ são exponenciais de operadores de um corpo, então o numerador da expressão (37) é dado pelo overlap entre determinantes de Slater. A técnica que usaremos para o cálculo de operadores entre estados determinantais é descrita no apêndice da referência (18). A partir da análise feita nesta referência segue que, se $|\phi\rangle$ e $|\tilde{\phi}\rangle$ são determinantes de Slater construídos a partir de estados de partícula independente $\left|\phi_{\alpha}\right\rangle$ e $\left|\tilde{\phi}_{\beta}\right\rangle$ respectivamente, então o overlap entre $|\phi\rangle$ e $|\tilde{\phi}\rangle$ é dado por:

$$
\langle\phi \mid \tilde{\phi}\rangle=\operatorname{det} \mathrm{B}
$$

onde os elementos da matriz B são dados abaixo:

$$
\text { (B) }{ }_{\alpha \beta}=\left\langle\phi_{\alpha}\right| \tilde{\phi}_{\beta}>
$$

onde no rótulo $\alpha=\mathrm{i} ; \mathrm{a}$ do estado de partícula independente "i" indica o estado orbital ocupado e "a" o estado de spin isospin correspondente. O rótulo $\beta=\mathrm{j} ; \mathrm{b}$ é interpretado de maneira análoga.

No caso específico do numerador da expressão (37), temos que:

$$
\begin{aligned}
& <\overrightarrow{\mathrm{x}} ; \sigma \tau \mid \phi_{\alpha}>=\mathrm{e}^{\tilde{\beta}^{\prime} \mathrm{A}^{+\mathrm{e}}} \phi_{\mathrm{i}}(\overrightarrow{\mathrm{x}}) \xi_{\mathrm{a}} \\
& <\overrightarrow{\mathrm{x}} ; \sigma \tau \mid \tilde{\phi}_{\beta}>=\mathrm{R}^{\mathrm{e}}(\Omega) \mathrm{e}^{\tilde{\beta} \mathrm{A}^{+\mathrm{e}}} \phi_{\mathrm{j}}(\overrightarrow{\mathrm{x}}) \xi_{\mathrm{b}}
\end{aligned}
$$

onde os operadores acima se referem a uma única partícula $\phi_{\mathrm{j}}(\overrightarrow{\mathrm{x}}), \phi_{\mathrm{j}}(\overrightarrow{\mathrm{x}})$ corresponde às 
funções de onda do oscilador harmônico e $\xi_{\mathrm{a}}=(\mathrm{n}+, \mathrm{n}-, \mathrm{p}+, \mathrm{p}-)$ a função de onda no espaço de spin-isospin. O cálculo do denominador da expressão (37), não apresenta grandes dificuldades uma vez que consiste no cálculo de um operador entre funções de onda do estado fundamental do oscilador harmônico. Por intermédio do procedimento discutido acima, obtemos a expressão (37) no caso do overlap e através da expressão (32) os elementos de matriz do overlap para os casos de interesse. No apêndice 1 discutimos os detalhes de tais cálculos.

\subsubsection{Cálculo do Potencial Através do MFG}

Nesta seção vamos discutir o procedimento que utilizaremos no cálculo dos elementos de matriz do potencial. Como nas seções anteriores, trataremos de modo unificado os submodelos $\mathrm{Sp}_{11}(1, \mathrm{R}), \mathrm{Sp}_{\perp}(1, \mathrm{R})$.

A energia potencial $\mathrm{V}$ é dada por:

$$
V=\sum_{i<j} V_{i j}
$$

onde utilizaremos a interação de dois corpos $\mathrm{V}_{\mathrm{ij}}$ da forma:

$$
\mathrm{V}_{\mathrm{ij}}=\exp \left[-\frac{\mathrm{r}_{\mathrm{ij}}^{2}}{\mu^{2}}\right]\left[\mathrm{V}_{0}+\mathrm{V}_{\mathrm{M}} \mathrm{P}_{\mathrm{ij}}^{\mathrm{e}}\right]
$$

onde $\mathrm{V}_{0}, \mathrm{~V}_{\mathrm{M}}$ são parâmetros do potencial; $\mathrm{P}_{\mathrm{ij}}^{\mathrm{e}}$ corresponde ao operador de troca de coordenadas espaciais dos nucleons "i" e "j", $r_{i j}=|\vec{x}(i)-\vec{x}(j)|$ e $\mu$ é o alcance do potencial. 
As forças de Brink-Boeker ${ }^{(19)}$, que são freqüentemente utilizadas no cálculo de núcleos leves podem ser parametrizados na forma acima. A fim de obtermos os elementos de matriz de V, utilizando o MFG, precisamos calcular a expressão em que $\hat{O}=\mathrm{V}$. De maneira aláloga à seção anterior, onde discutimos o cálculo do overlap, a partir das expressões (35) e (36) obtemos:

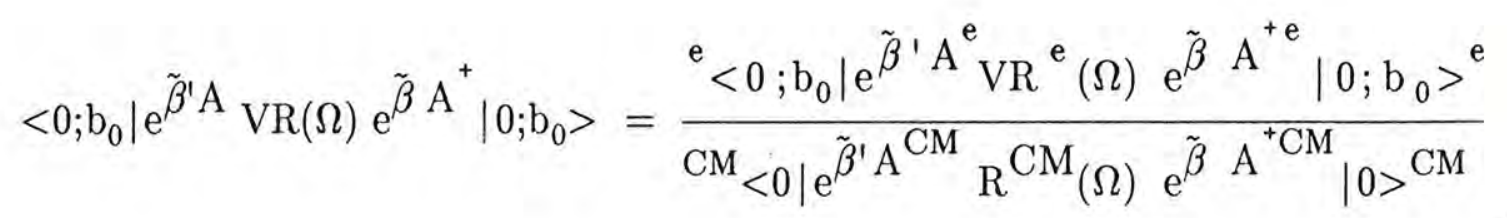

onde $\mathrm{R}^{\mathrm{e}}(\Omega)=\mathrm{R}(\Omega) \mathrm{R}^{\mathrm{CM}}(\Omega)$ e, V é dado em termos de coordenadas intrínsecas.

O denominador da expressão acima não apresenta problemas para seu cálculo como discutimos na seção anterior e portanto vamos apenas nos preocupar com o seu numerador. O numerador em todos os casos que serão considerados, se reduz a um ou uma soma de termos que envolvem o cálculo do operador de dois corpos $\mathrm{V}$ entre estados determinantais. O cálculo do operador $\mathrm{V}$ entre estados determinantais é feito utilizando-se a técnica descrita na referência (18) que resulta na seguinte expressão para o elemento de matriz do operador $\mathrm{V}$ entre os estados determinantais $|\phi\rangle$ e $|\tilde{\phi}\rangle$ :

$$
<\phi|\mathrm{V}| \tilde{\phi}>=\frac{1}{2} \sum_{\alpha \beta \gamma \delta}<\phi_{\alpha} \phi_{\beta}\left|\mathrm{V}_{12}\right| \tilde{\phi}_{\gamma} \tilde{\phi}_{\delta}>\mathrm{C}_{\alpha \beta \gamma \delta}
$$

onde usamos a mesma notação da seção 4.4 .2 e $\mathrm{C}_{\alpha \beta \gamma \delta}$ é por definição o duplo cofator associado à matriz $\mathrm{B}$ do overlap. Usando este procedimento, podemos calcular a expressão (39), que substituímos em (33) para por fim através de (32), obtermos os 
elementos de matriz do potencial nos casos de interesse. Os detalhes envolvidos em tais cálculos, discutimos no apêndice 1 .

\subsubsection{Cálculo Algébrico da Energia Cinética}

Temos por definição que:

$$
\mathrm{T}=\left[\sum_{\mathrm{k}=1}^{\mathrm{A}} \mathrm{T}(\mathrm{k})\right]-\mathrm{T}_{\mathrm{CM}}=\sum_{\mu=1}^{3} \sum_{\mathrm{k}=1}^{\mathrm{A}-1} \frac{\pi_{\mu}(\mathrm{k})^{2}}{2 \mathrm{~m}}
$$

onde $T(k)$ é o operador energia cinética do k-ésimo nucleon em relação a um referencial fixo no espaço e $\mathrm{T}_{\mathrm{CM}}$ corresponde à energia cinética do centro de massa. A partir da definição do operador criação e aniquilação de quanta de oscilador:

$$
\begin{aligned}
& \mathrm{a}_{\mu ; \mathrm{k}}^{+}=\frac{1}{\sqrt{2}}\left[\frac{\rho_{\mu}(\mathrm{k})}{\mathrm{b}_{0}}-\frac{\mathrm{ib}_{0}}{\hbar} \pi_{\mu}(\mathrm{k})\right] \\
& \mathrm{a}_{\mu ; \mathrm{k}}=\left[\mathrm{a}_{\mu ; \mathrm{k}}^{+}\right]^{+}
\end{aligned}
$$

obtemos:

$$
\begin{aligned}
& \pi_{\mu}(\mathrm{k})=\frac{\mathrm{i} \hbar}{\mathrm{b}_{0} \sqrt{2}}\left[\mathrm{a}_{\mu ; \mathrm{k}}^{+}-\mathrm{a}_{\mu ; \mathrm{k}}\right] ; \mathrm{k}=1, \ldots, \mathrm{A}-1 \\
& \rho_{\mu}(\mathrm{k})=\frac{\mathrm{b}_{0}}{\sqrt{2}}\left[\mathrm{a}_{\mu ; \mathrm{k}}^{+}+\mathrm{a}_{\mu ; \mathrm{k}}\right] .
\end{aligned}
$$


Substituindo $\pi_{\mu}(\mathrm{k})$ dado acima, na definição de $\mathrm{T}$, obtemos:

$$
\mathrm{T}=\frac{\hbar^{2}}{2 m b_{0}^{2}}\left[2\left[\mathrm{~A}_{0 \prime}+\mathrm{A}_{0_{\perp}}\right]-\left[\mathrm{A}_{\|}^{+}+\mathrm{A}_{\perp}^{+}\right]-\left[\mathrm{A}_{\|}+\mathrm{A}_{\perp}\right]\right]
$$

onde usamos a definição dos operadores das álgebras $\operatorname{sp}_{\perp}(1, R)$ e $\operatorname{sp}_{11}(1, R)$ dados na seções 4.2.1 e 4.2.2. Como os operadores $\mathrm{T},\left[\mathrm{A}_{0^{\prime \prime}}+\mathrm{A}_{0_{\perp}}\right],\left[\mathrm{A}_{11}+\mathrm{A}_{\perp}\right],\left[\mathrm{A}_{11}^{+}+\mathrm{A}_{\perp}^{+}\right]$são escalares por rotação, é imediato que o elemento de matriz de $\mathrm{T}$ na base de estados (24) é dado por:

$$
\begin{aligned}
& <n^{\prime}, L^{\prime} M^{\prime} K^{\prime} ; b_{0}|T| n, L M K ; b_{0}>=\delta_{L L^{\prime}} \delta_{M^{\prime}} \frac{\hbar^{2}}{2 m_{0}^{2}} \cdot \\
& \cdot\left\{2<n^{\prime} ; b_{0}\left|P_{K^{\prime} K}^{L}\left[A_{0^{\prime \prime}}+A_{0_{\perp}}\right]\right| n ; b_{0}>-<n^{\prime} ; b_{0}\left|\left[A_{1 \prime}^{+}+A_{\perp}^{+}\right] P_{K^{\prime} K}^{L}\right| n ; b_{0}>-\right. \\
& \left.-<n^{\prime} ; b_{0}\left|P_{K^{\prime} K}^{L}\left[A_{\prime \prime}+A_{\perp}\right]\right| n ; b_{0}>\right\}
\end{aligned}
$$

onde usamos a propriedade do projetor em momento angular dada na expressão (31) para obtermos a expressão acima.

\subsubsection{Cálculo Algébrico do Operador Momento de Monopolo}

O operador momento de monopolo é definido em relação a um referencial fixo no espaço por:

$$
M^{\mathrm{e}}=\sum_{\mathrm{k}=1}^{\mathrm{A}} \sum_{\mu=1}^{3} \mathrm{x}_{\mu}(\mathrm{k})^{2} .
$$


Fazendo a transformação ortogonal que decompõe o operador acima em suas componentes intrínseca e de centro de massa, como mostramos no capítulo 2, obtemos:

$$
M=M^{\mathrm{e}}-\mathrm{M}^{\mathrm{CM}}=\sum_{\mathrm{k}=1}^{\mathrm{A}-1} \sum_{\mu=1}^{3} \rho_{\mu}(\mathrm{k})^{2} .
$$

Usando as relações (41) obtemos:

$$
M=b_{0}^{2}\left[2\left[A_{0 \prime \prime}+A_{0_{\perp}}\right]+\left[A_{11}^{+}+A_{\perp}^{+}\right]+\left[A_{11}+A_{\perp}\right]\right]
$$

como $M,\left[\mathrm{~A}_{0^{\prime \prime}}+\mathrm{A}_{0_{\perp}}\right],\left[\mathrm{A}_{11}+\mathrm{A}_{\perp}\right],\left[\mathrm{A}_{11}^{+}+\mathrm{A}_{\perp}^{+}\right]$são escalares por rotação e temos a propriedade (31) do projetor, segue que:

$$
\begin{aligned}
& <n^{\prime}, L^{\prime} M^{\prime} K^{\prime} ; b_{0}|M| n, L M K ; b_{0}>=\delta_{L^{\prime}} \delta_{M M^{\prime}} b_{0}^{2} . \\
& \cdot\left\{2<n^{\prime} ; b_{0}\left|P_{K^{\prime} K}^{L}\left[A_{0^{\prime \prime}}+A_{0_{\perp}}\right]\right| n ; b_{0}>+<n^{\prime} ; b_{0}\left|\left[A_{\prime^{\prime \prime}}^{+}+A_{\perp}^{+}\right] P_{K^{\prime} K}^{L}\right| n ; b_{0}>+\right. \\
& \left.+<n^{\prime} ; b_{0}\left|P_{K^{\prime} K}^{L}\left[A_{1 \prime}+A_{\perp}\right]\right| n ; b_{0}>\right\} .
\end{aligned}
$$

Para obtermos o operador momento de monopolo elétrico, definido por

$$
\mathrm{M}_{\mathrm{el}}^{\mathrm{e}}=\sum_{\mathrm{k}=1}^{\mathrm{A}} \sum_{\mu=1}^{3} \mathrm{x}_{\mu}(\mathrm{k})^{2} \mathrm{e}\left[\frac{1}{2}-\mathrm{t}_{\mathrm{z}}(\mathrm{k})\right]
$$

lembramos que todos os estados que vamos utilizar tem $\mathrm{T}=0$ e portanto para obtermos os elementos de matriz de $\mathrm{M}_{\mathrm{el}}$ basta multiplicarmos por (e/2) a expressão (43). 


\subsubsection{Cálculo Algébrico do Operador Momento de Quadrupolo}

O operador momento de quadrupolo é um operador tensorial de ordem 2, cujas componentes são definidas por:

$$
\mathrm{Q}_{2 \mu}^{\mathrm{e}}=\sum_{\mathrm{k}=1}^{\mathrm{A}} \mathrm{r}(\mathrm{k})^{2} \mathrm{Y}_{2 \mu}\left(\Omega_{\mathrm{k}}\right)
$$

Fazendo a trasformação para coordenadas relativas e de centro de massa, como mostramos no capítulo 2, obtemos:

$$
\mathrm{Q}_{2 \mu}^{\mathrm{e}}=\mathrm{Q}_{2 \mu}+\mathrm{Q}_{2 \mu}^{\mathrm{CM}}
$$

onde

$$
\mathrm{Q}_{2 \mu}=\sqrt{\frac{5}{16 \pi}} \mathrm{q}_{2 \mu}
$$

e os operadores $q_{2 \mu}$ são dados por:

$$
\begin{aligned}
& \mathrm{q}_{20}=\sum_{\mathrm{k}=1}^{A-1}\left[2 \rho_{1}(\mathrm{k})^{2}-\rho_{2}(\mathrm{k})^{2}-\rho_{3}(\mathrm{k})^{2}\right] \\
& \mathrm{q}_{2 \pm 1}=\mp \sqrt{6} \sum_{\mathrm{k}=1}^{\mathrm{A}-1} \rho_{1}(\mathrm{k})\left[\rho_{2}(\mathrm{k}) \pm \mathrm{i} \rho_{3}(\mathrm{k})\right] \\
& \mathrm{q}_{2 \pm 2}=\sqrt{\frac{3}{2}} \sum_{\mathrm{k}=1}^{\mathrm{A}-1}\left[\rho_{2}(\mathrm{k}) \pm \mathrm{i} \rho_{3}(\mathrm{k})\right]^{2} .
\end{aligned}
$$


Para obtermos uma expressão que nos permite o cálculo do operador $\mathrm{Q}_{2 \mu}$ na base de estados (24) vamos utilizar o procedimento descrito na referência 20 , que discutiremos de maneira abreviada abaixo. A partir da definição dos operadores criação e aniquilição de quanta de oscilador na base esférica dada a seguir:

$$
\begin{aligned}
& \mathrm{b}_{1 ; \mathrm{k}}^{+}=-\frac{\mathrm{i}}{\sqrt{2}}\left[\mathrm{a}_{2 ; \mathrm{k}}^{+}+\mathrm{ia}_{3 ; \mathrm{k}}^{+}\right] \\
& \mathrm{b}_{0 ; \mathrm{k}}^{+}=\mathrm{i} \mathrm{a} \mathrm{a}_{1 ; \mathrm{k}}^{+} \\
& \mathrm{b}_{-1 ; \mathrm{k}}^{+}=\frac{\mathrm{i}}{\sqrt{2}}\left[\mathrm{a}_{2 ; \mathrm{k}}^{+}-\mathrm{ia}_{3 ; \mathrm{k}}^{+}\right]
\end{aligned}
$$

e

$$
\tilde{\mathrm{b}}_{\mu ; \mathrm{k}}=(-1)^{1-\mu} \mathrm{b}_{-\mu ; \mathrm{k}}, \text { onde } \quad \mathrm{b}_{\mu ; \mathrm{k}}=\left(\mathrm{b}_{\mu ; \mathrm{k}}^{+}\right)^{+}, \mathrm{k}=1, \ldots, \mathrm{A}-1
$$

definimos os seguintes operadores tensoriais:

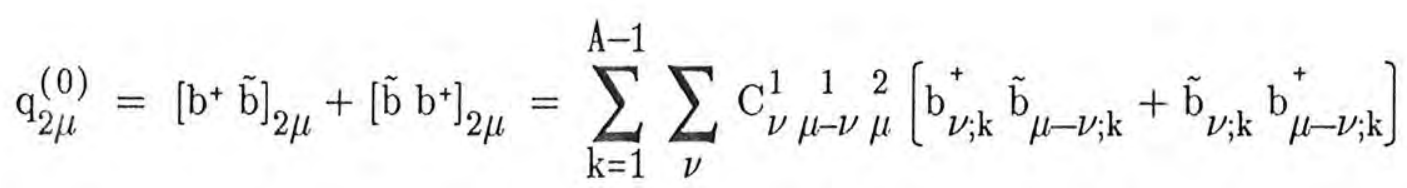

e de maneira análoga

$$
\begin{aligned}
& \mathrm{q}_{2 \mu}^{(+)}=\left[\mathrm{b}^{+} \mathrm{b}^{+}\right]_{2 \mu} \\
& \mathrm{q}_{2 \mu}^{(-)}=[\tilde{\mathrm{b}} \tilde{\mathrm{b}}]_{2 \mu} .
\end{aligned}
$$


Calculando explicitamente os operadores $\mathrm{q}_{2 \mu}^{(0)}, \mathrm{q}_{2 \mu}^{(+)}, \mathrm{q}_{2 \mu}^{(-)}$acima obtemos:

$$
\mathrm{q}_{2 \mu}=-\sqrt{\frac{3}{2}} \mathrm{~b}_{0}^{2}\left[\mathrm{q}_{2 \mu}^{(0)}+\mathrm{q}_{2 \mu}^{(+)}+\mathrm{q}_{2 \mu}^{(-)}\right]
$$

onde

$$
\begin{aligned}
& \mathrm{q}_{20}^{(0)}=-\sqrt{\frac{2}{3}}\left[2 \mathrm{C}_{11}-\mathrm{C}_{22}-\mathrm{C}_{33}\right] \\
& \mathrm{q}_{2 \pm 1}^{(0)}= \pm\left[\mathrm{C}_{12}+\mathrm{C}_{21} \pm \mathrm{i}\left[\mathrm{C}_{13}+\mathrm{C}_{31}\right]\right] \\
& \mathrm{q}_{2 \pm 2}^{(0)}=-\mathrm{C}_{22}+\mathrm{C}_{33} \mp \mathrm{i}\left[\mathrm{C}_{23}+\mathrm{C}_{32}\right] \\
& \mathrm{q}_{20}^{(+)}=-\sqrt{\frac{2}{3}}\left[2 \mathrm{~A}_{11}^{+}-\mathrm{A}_{22}^{+}-\mathrm{A}_{33}^{+}\right] \\
& \mathrm{q}_{2 \pm 1}^{(+)}= \pm 2\left[\mathrm{~A}_{12}^{+} \pm \mathrm{i} \mathrm{A}_{13}^{+}\right] \\
& \mathrm{q}_{2 \pm 2}^{(+)}=-\mathrm{A}_{22}^{+}+\mathrm{A}_{33}^{+} \mp 2 \mathrm{i} \mathrm{A}_{23}^{+} \\
& \mathrm{q}_{20}^{(-)}=-\sqrt{\frac{2}{3}}\left[2 \mathrm{~A}_{11}-\mathrm{A}_{22}-\mathrm{A}_{33}\right] \\
& \mathrm{q}_{2 \pm 1}^{(-)}= \pm 2\left[\mathrm{~A}_{12} \pm \mathrm{i} \mathrm{A}_{13}\right] \\
& \mathrm{q}_{2 \pm 2}^{(-)}=-\mathrm{A}_{22}+\mathrm{A}_{33} \mp 2 \mathrm{i} \mathrm{A}_{23} .
\end{aligned}
$$

A fim de calcularmos os elementos de matriz de $Q_{2 \mu}$ na base de estados (24), é conveniente que o operador $q_{2 \mu}^{(+)}$atue à esquerda e $q_{2 \mu}^{(-)}, q_{2 \mu}^{(0)}$ atuem à direita uma vez que $\mathrm{q}_{2 \mu}^{(-)}$possue apenas operadores que aniquilam 2 quantas de oscilador. 
Usando as seguintes propriedades do projetor $\mathrm{P}_{\mathrm{MK}}^{\mathrm{L}}$ :

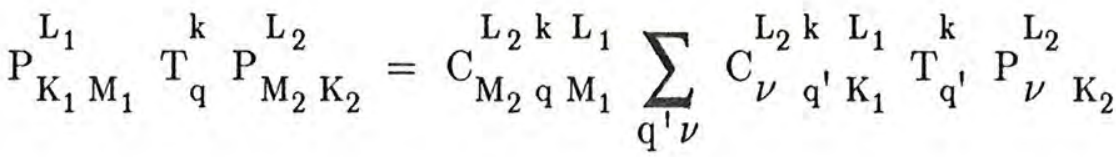

$$
\begin{aligned}
& \mathrm{P}_{\mathrm{K}_{1} \mathrm{M}_{1}}^{\mathrm{L}_{1}} \mathrm{~T}_{\mathrm{q}}^{\mathrm{k}} \mathrm{P}_{\mathrm{M}_{2} \mathrm{~K}_{2}}^{\mathrm{L}_{2}}=\frac{\left(2 \mathrm{~L}_{2}+1\right)}{\left(2 \mathrm{~L}_{1}+1\right)} \mathrm{C}_{\mathrm{M}_{2} \mathrm{q} \mathrm{M}_{1}}^{\mathrm{L}_{2} \mathrm{k} \mathrm{L}_{1}} \sum_{\mathrm{q}^{\prime} \nu} \mathrm{C}_{\mathrm{K}_{2} \mathrm{q}^{\prime} \nu}^{\mathrm{L}_{2} \mathrm{k} \mathrm{L}_{1}} \mathrm{P}_{\mathrm{K}_{1} \nu}^{\mathrm{L}_{1}} \mathrm{~T}_{\mathrm{q}^{\prime}}^{\mathrm{k}}
\end{aligned}
$$

e a expressão (45) obtemos:

$$
\begin{aligned}
& <\mathrm{n}^{\prime}, \mathrm{L}^{\prime} \mathrm{M}^{\prime} \mathrm{K}^{\prime} ; \mathrm{b}_{0}\left|\mathrm{Q}_{2 \mu}\right| \mathrm{n}, \mathrm{LMK} ; \mathrm{b}_{0}>=\left\langle\mathrm{n}^{\prime} ; \mathrm{b}_{0}\left|\mathrm{P}_{\mathrm{K}^{\prime} \mathrm{M}^{\prime}}^{\mathrm{L}^{\prime}} \mathrm{Q}_{2 \mu} \mathrm{P}_{\mathrm{MK}}^{\mathrm{L}}\right| \mathrm{n} ; \mathrm{b}_{0}>=\right. \\
& =-\sqrt{\frac{15}{32 \pi}} \mathrm{b}_{0}^{2} \mathrm{C}_{\mathrm{M} \mu \mathrm{M}^{\prime}}^{\mathrm{L} 2 \mathrm{~L}^{\prime}} \cdot\left\{\sum_{\mathrm{q}^{\prime}} \mathrm{C}_{\mathrm{K}^{\prime}-\mathrm{q}^{\prime} \mathrm{q}^{\prime} \mathrm{K}^{\prime}}^{\mathrm{L}} \quad \mathrm{Ln}^{\prime} ; \mathrm{b}_{0}\left|\mathrm{q}_{2 \mathrm{q}^{\prime}}^{(+)} \mathrm{P}_{\mathrm{K}^{\prime}-\mathrm{q}^{\prime} \mathrm{K}}^{\mathrm{L}}\right| \mathrm{n} ; \mathrm{b}_{0}>+\right.
\end{aligned}
$$

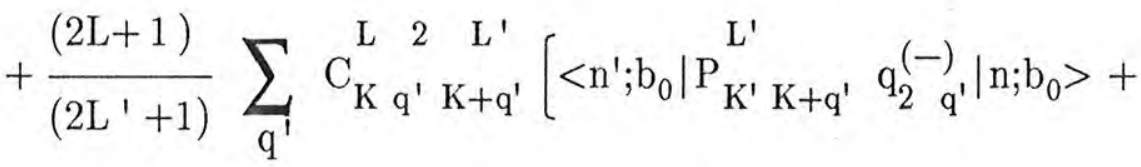

$$
\begin{aligned}
& \left.\left.+<\mathrm{n}^{\prime} ; \mathrm{b}_{0}\left|\mathrm{P}_{\mathrm{K}^{\prime} \mathrm{K}+\mathrm{q}^{\prime}}^{\mathrm{L}^{\prime}} \mathrm{q}_{2}^{(0)} \mathrm{q}^{\prime}\right| \mathrm{n} ; \mathrm{b}_{0}>\right]\right\} \text {. }
\end{aligned}
$$

A expressão acima será usada nos casos de interesse. 


\subsection{Resultados Numéricos}

Nesta seção vamos analisar os resultados numéricos associados ao ${ }^{12} \mathrm{C}$, utilizando os submodelos $\mathrm{Sp}(1, \mathrm{R})$ determinados pelos estados de peso mínimo op-ob, $2 \mathrm{p}-2 \mathrm{~b}, 4 \mathrm{p}-4 \mathrm{~b}$, que discutimos nas seções anteriores.

Os elementos de matriz dos operadores de interesse na base de estados do modelo $\mathrm{Sp}(1, \mathrm{R})$, foram calculados utilizando-se o procedimento que discutimos na seção 4.4 onde deixamos os detalhes especificos para o apêndice 1 .

Em todos os nossos cálculos utilizaremos a interação semi-microscópica B1, de Brink-Boeker ${ }^{(19)}$.

\subsubsection{Submodelo $\mathrm{Sp}_{\perp}(1, \mathrm{R})$ Determinado pelo Estado de Peso Mínimo op-ob}

Os níveis de energia e funções de onda associados pelo modelo ao ${ }^{12} \mathrm{C}$, são obtidos pela diagonalização da hamiltoniana na base de estados (12), para cada valor do momento angular orbital L. O número máximo de estados de base considerados $\mathrm{N}$ é fixado de modo a obtermos convergência para todos os estados de interesse, de acordo com o critério que enunciamos a seguir.

Afirmamos que é alcançada convergência quando, para todos os estados de interesse, aumentando a dimensão da base de $\mathrm{N}$ para $\mathrm{N}+1$ estados, a alteração na energia obtida pela diagonalização de $\mathrm{H}$ é menor que um certo $\varepsilon$, fixado a priori. Na prática utilizamos $\varepsilon=0.0001$ e uma base com $N=34$ foi suficiente para obtermos a convergência desejada. Os elementos de matriz da hamiltoniana $\mathrm{H}$ dependem do comprimento do oscilador $\mathrm{b}_{0}$. A partir da minimização da energia do estado fundamental obtido pela diagonalização de $\mathrm{H}$, fixamos o parâmetro $\mathrm{b}_{0}$. A função de onda do modelo é obtida pela expansão na base 
de estados (12), que representamos da maneira seguinte:

$$
\left|\nu, \mathrm{LM} ; \mathrm{b}_{0}\right\rangle=\sum_{\mathrm{n}=0}^{\mathrm{N}} \mathrm{C}_{\mathrm{n}}^{\nu}(\mathrm{L}) \frac{\left|\mathrm{n}(04), \mathrm{LM} 0 ; \mathrm{b}_{0}\right\rangle}{\left\langle\mathrm{n}(04), \mathrm{LM} 0 ; \mathrm{b}_{0} \mid \mathrm{n}(04), \mathrm{LM} 0 ; \mathrm{b}_{0}\right\rangle^{1 / 2}}
$$

Na figura 4.1 mostramos os níveis de energia de excitação associados pelo modelo ao núcleo ${ }^{12} \mathrm{C}$, que obtivemos. Os nossos resultados estão de acordo com os obtidos na referência (4), que realiza um cálculo análogo ao que fizemos. Na figura 4.2 mostramos os coeficientes $\mathrm{C}_{\mathrm{n}}^{\nu}(\mathrm{L})$ da função de onda, (48), para os três primeiros estados $0^{+}$que obtivemos pela diagonalização de $\mathrm{H}$. Através dos pontos relativos a cada um desses estados $0^{+}$traçamos uma linha contínua unindo-os.

A figura 4.1 nos mostra que a medida que vamos considerando bandas mais excitadas, os níveis de energia vão ficando mais comprimidos e os $\mathrm{B}(\mathrm{E} 2)$ intra-banda correspondentes vão crescendo, o que mostra uma maior deformação. A figura 4.2 nos mostra que a função de onda tem componentes não desprezíveis para um grande número de estados de base do modelo. Por exemplo, note que mesmo para o estado fundamental o estado de base mais provável não é o estado com $n=0$. Também note que para o terceiro estado $0^{+}$estados altamente excitados tem probabilidades comparáveis às de $n=0$. Este comportamento também é observado em cálculos do tipo $\mathrm{Sp}(1, \mathrm{R})$ relativos aos núcleos ${ }^{20} \mathrm{Ne}$ e ${ }^{18} \mathrm{O}$ realizados pelos autores das referências 7 e 8.

A fim de demonstrarmos a importância das configurações excitadas, mostramos nas figuras 4.3-4.5 a contribuição parcial dos estados de base ao momento de quadrupolo elétrico do primeiro estado $2^{+}$e ao $\mathrm{B}(\mathrm{E} 2,2 \rightarrow 0)$ da banda fundamental e primeira banda excitada. Nesses gráficos, o valor das grandezas considerando estados até n, é dado pela soma das alturas dos blocos anteriores mais o bloco que começa no ponto n. Observando essas figuras é evidente a importância dos estados excitados. 


\section{NÍVEIS DE ENERGIA (REPRESENTAÇÃO OP-OB)}

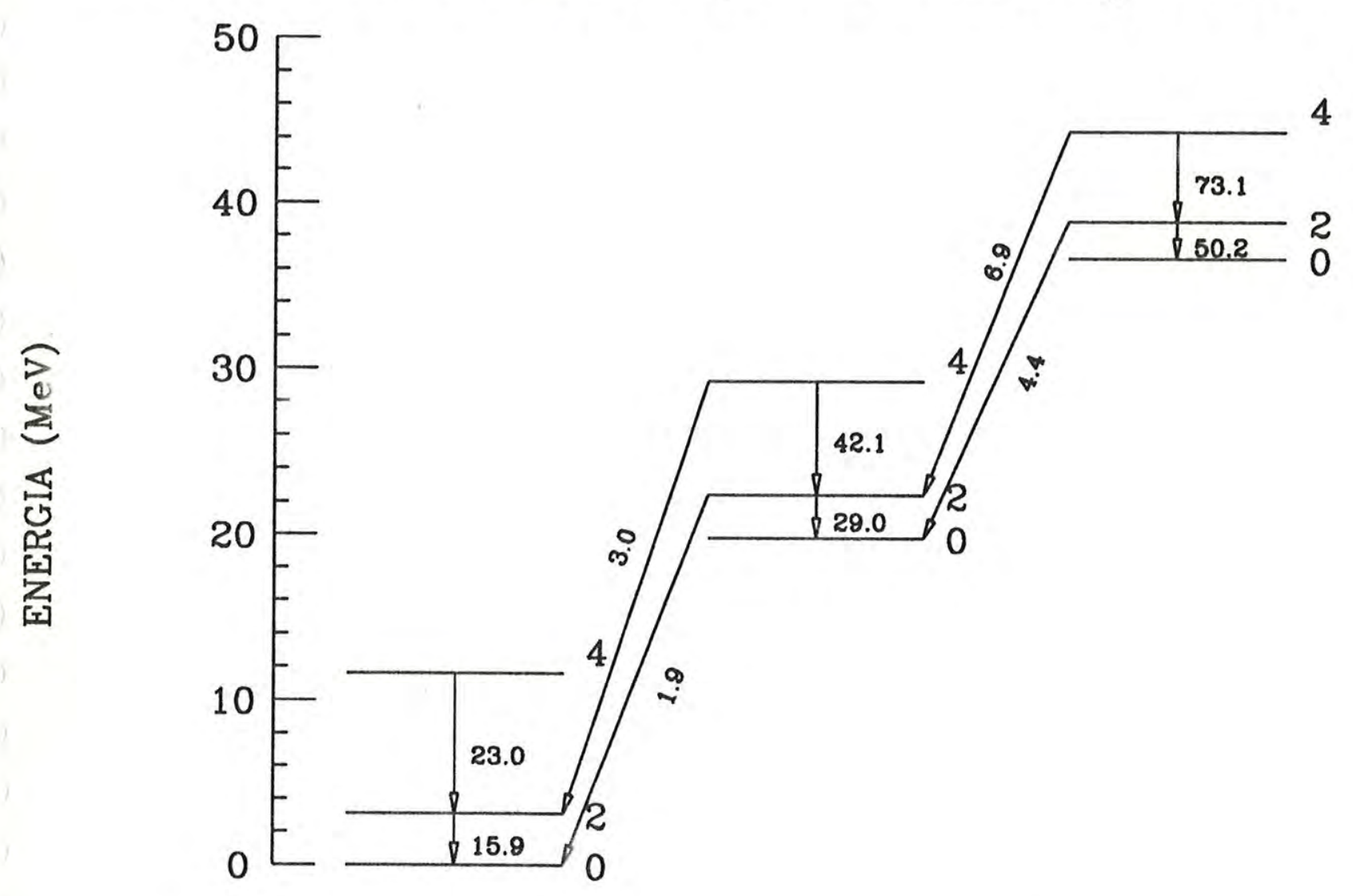

Fig. 4.1. Níveis de energia de excitação e $B(E 2)$ calculados no modelo $\operatorname{Sp}_{\perp}(1, \mathrm{R})$ associado ao estado de peso mínimo $0 \mathrm{p}-0 \mathrm{~b}$. Energia de ligação B.E. $=72.7 \mathrm{MeV}$ e $\mathrm{b}_{0}=1.36 \mathrm{fm}$. (Energias em MeV e B(E2) em e $\mathrm{e}^{2} \cdot \mathrm{fm}^{4}$ ) 


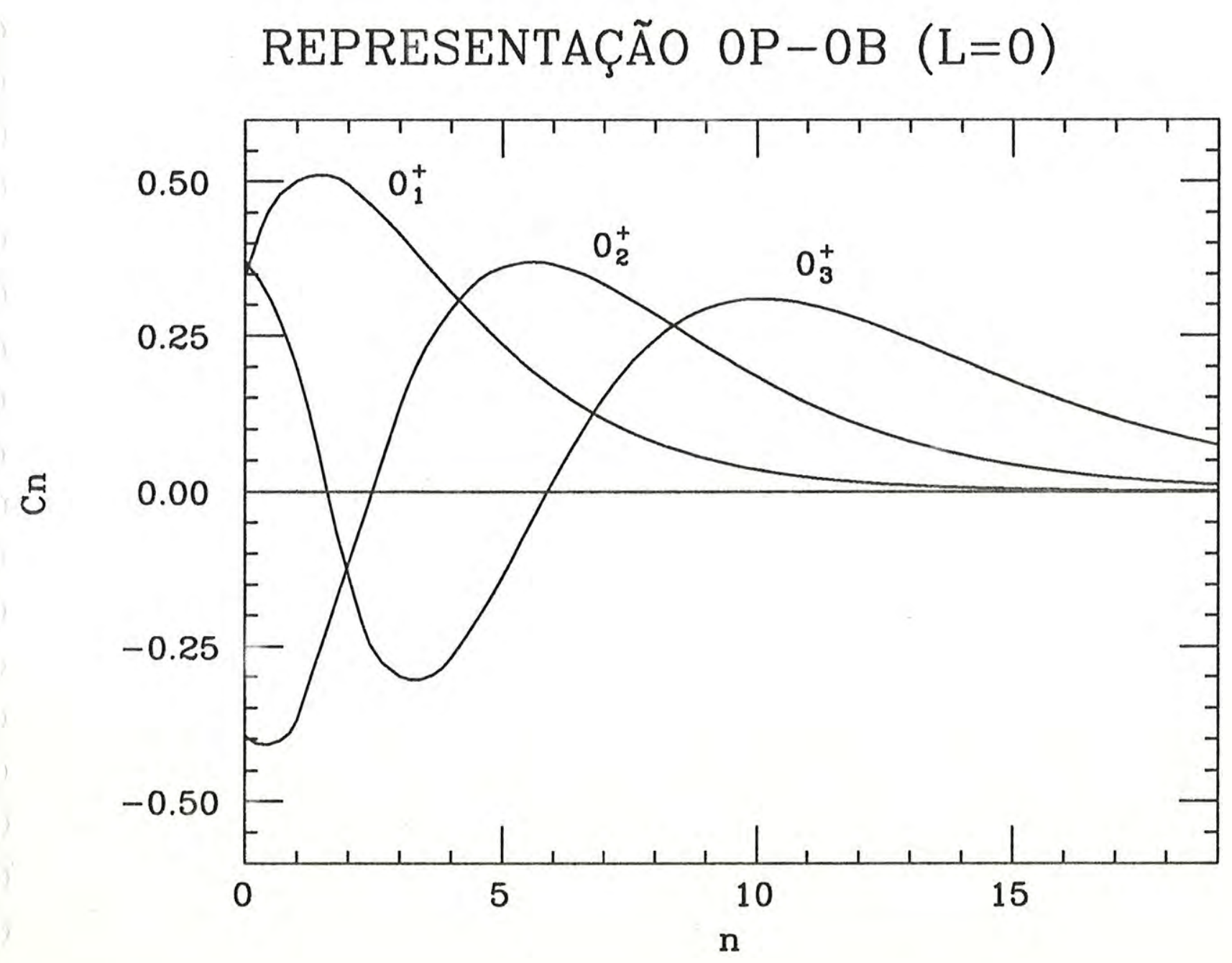

Fig. 4.2. Valores dos coeficientes $\mathrm{C}_{\mathrm{n}}^{\nu}(\mathrm{L})$ para os três primeiros estados $0^{+}$, como função de $\mathrm{n}$, calculados no modelo $\mathrm{Sp}_{\perp}(1, \mathrm{R})$ associado ao estado de peso mínimo $0 \mathrm{p}-0 \mathrm{~b}$. 


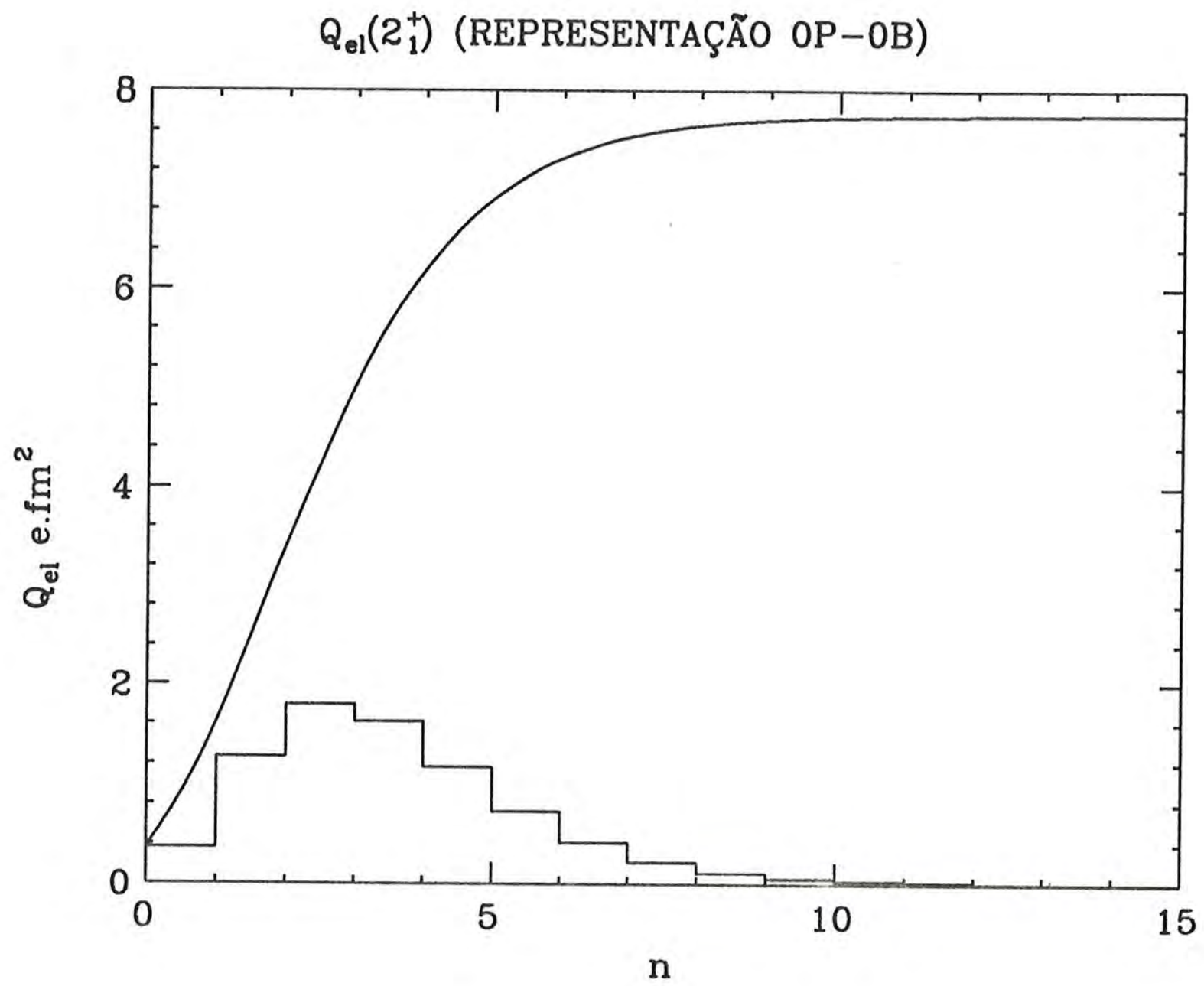

Fig. 4.3. Contribuição parcial ao $\mathrm{Q}_{\mathrm{el}}\left(2_{1}^{+}\right)$devido à inclusão sistemática dos estados de base do modelo $\mathrm{Sp}_{\perp}(1, \mathrm{R})$ na expansão da função de onda dada pela eq.(4.48). O valor de $\mathrm{Q}_{\mathrm{el}}\left(2_{1}^{+}\right)$no ponto $\mathrm{n}$, é obtido considerando-se na expansão (48), a contribuição de todos os termos até $n$, inclusive. 


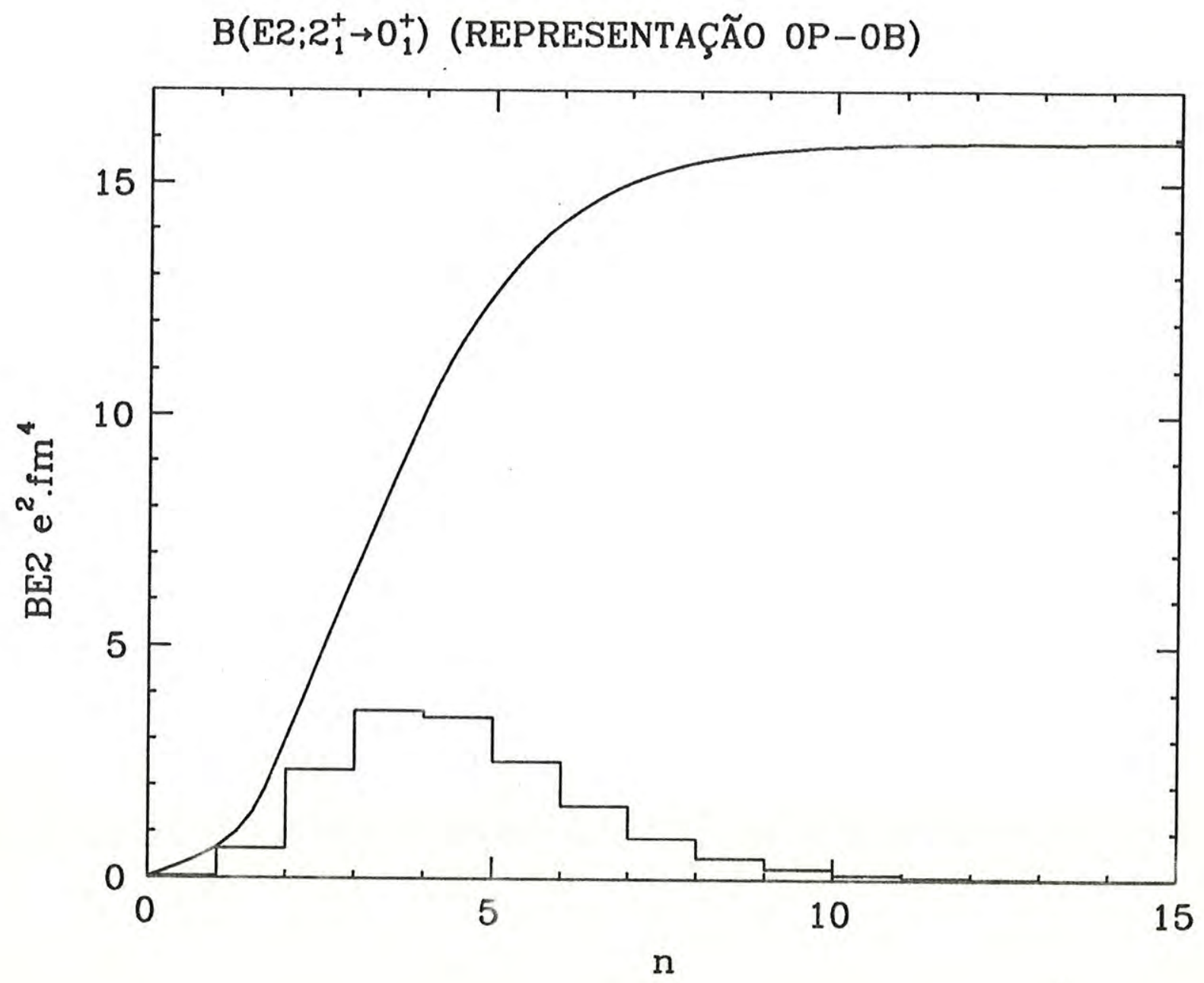

Fig. 4.4. Contribuição parcial ao $\mathrm{B}\left(\mathrm{E} 2,2_{1}^{+} \rightarrow 0_{1}^{+}\right)$devido à inclusão sistemática dos estados de base do modelo $\mathrm{Sp}_{\perp}(1, \mathrm{R})$ na expansão (4.48) da função de onda. $\mathrm{O}$ valor do $\mathrm{B}(\mathrm{E} 2)$ no ponto $\mathrm{n}$,é obtido considerando-se na expansão (48), a contribuição de todos os termos até $n$, inclusive. 


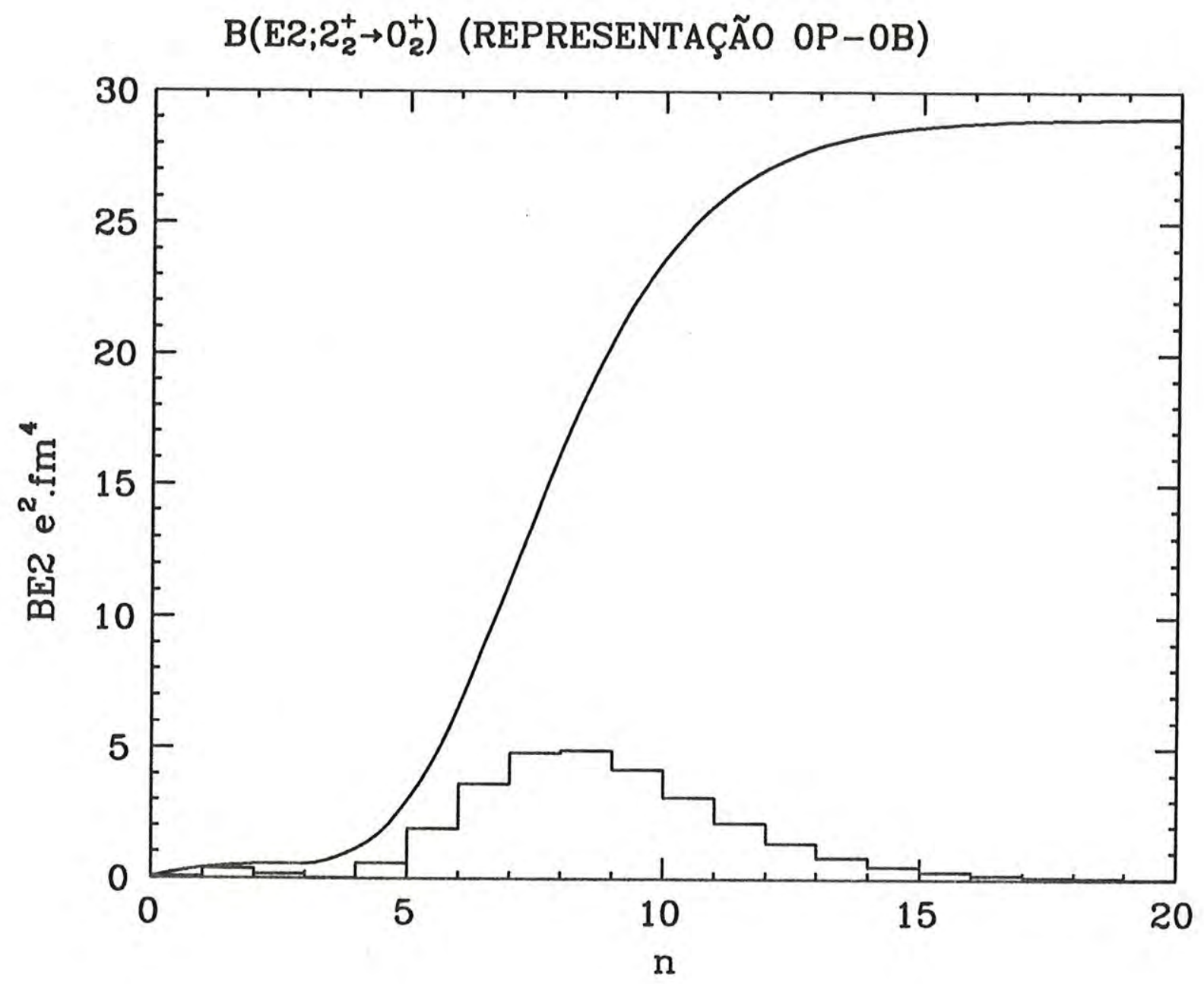

Fig. 4.5. Contribuição parcial ao $\mathrm{B}\left(\mathrm{E} 2,2_{2}^{+} \rightarrow 0_{2}^{+}\right)$devido à inclusão sistemática dos estados de base do modelo $\mathrm{Sp}_{\perp}(1, \mathrm{R})$ na expansão (4.48) da função de onda. $\mathrm{O}$ valor do $\mathrm{B}(\mathrm{E} 2)$ no ponto $\mathrm{n}$, é obtido considerando-se na expansão (48), a contribuição de todos os termos até $n$, inclusive. 
$\mathrm{Na}$ tabela 4.1 apresentamos os resultados obtidos para o raio quadrático médio $<\mathrm{r}^{2}>^{1 / 2}$ e momento de quadrupolo elétrico $\mathrm{Q}_{\mathrm{el}}$ para os estados de interesse. Os valores obtidos para os BE2 são apresentados na figura 4.1.

\begin{tabular}{rcc}
\hline $\mathrm{L}_{\nu}^{\pi}$ & $<\mathrm{r}^{2}>^{1 / 2}$ & $\mathrm{Q}_{\mathrm{el}}$ \\
\hline $0_{1}^{+}$ & 2.60 & - \\
$2_{1}^{+}$ & 2.59 & 7.75 \\
$4_{1}^{+}$ & 2.58 & 8.70 \\
$0_{2}^{+}$ & 2.90 & - \\
$2_{2}^{+}$ & 2.89 & 18.23 \\
$4_{2}^{+}$ & 2.88 & 20.58 \\
$0_{3}^{+}$ & 3.22 & - \\
$2_{3}^{+}$ & 3.22 & 32.01 \\
$4_{3}^{+}$ & 3.21 & 36.64 \\
\hline
\end{tabular}

Tab. 4.1. Propriedades geométricas dos estados calculados no modelo $\mathrm{Sp}_{\perp}(1, \mathrm{R})$, associado ao estado de peso mínimo op-ob $\cdot\left(<\mathrm{r}^{2}>^{1 / 2}\right.$ em $\mathrm{fm}, \mathrm{Q}_{\mathrm{el}}$ em $\left.\mathrm{e} \cdot \mathrm{fm}^{2}\right)$. 


\section{4..5.2. Submodelo $\mathrm{Sp}_{\| \prime}(1, \mathrm{R})$ Determinado pelo Estado de Peso Mínimo $2 \mathrm{p}-2 \mathrm{~b}$}

As características da base de estados do modelo $\operatorname{Sp}_{11}(1, R)$, associada ao estado de peso mínimo $2 \mathrm{p}-2 \mathrm{~b}$, discutimos na seção 4.3.2. Como vimos, neste caso temos uma base de estados $\left|\mathrm{n}(62), \mathrm{LMK} ; \mathrm{b}_{0}\right\rangle$, que são ortogonais no rótulo $\mathrm{n}$, mas no entanto não ortogonais no rótulo $\mathrm{K}$. Utilizando a regra de Elliott, vimos que só precisamos projetar estados com $\mathrm{K}=0$ ou $\mathrm{K}=2$. No caso específico do estado com $\mathrm{L}=0$, vimos que este estado só pode ser projetado de estados com $\mathrm{K}=0$ e portanto temos uma base ortogonal a priori, e assim não teremos problemas com a base.

Para obtermos os estados com $\mathrm{L}=2$ e $\mathrm{L}=4$, nós ortogonalizamos pelo procedimento de Gram-Schmidt obtendo a base ortogonalizada dada pelos estados seguintes:

$$
\begin{aligned}
\mid \mathrm{n}(62), \mathrm{LMk}=0>_{\mathrm{GS}}= & \mid \mathrm{n}(62), \mathrm{LMK}=0> \\
\mid \mathrm{n}(62), \mathrm{LMk}=2>_{\mathrm{GS}}= & \mid \mathrm{n}(62), \mathrm{LMK}=2>-\frac{<\mathrm{n}(62), \mathrm{LMK}=0 \mid \mathrm{n}(62), \mathrm{LMK}=2>}{<\mathrm{n}(62), \mathrm{LMK}=0 \mid \mathrm{n}(62), \mathrm{LMK}=0>} . \\
& \cdot \mid \mathrm{n}(62), \mathrm{LMK}=0>
\end{aligned}
$$

omitimos na notação acima o comprimento do oscilador $\mathrm{b}_{0}$.

Os níveis de energia e funções de onda são obtidos pela diagonalização da hamiltoniana na base de estados acima. Usando o mesmo critério de convergência da seção anterior, $N=34$ mais uma vez foi suficiente para obtermos a convergência desejada. Convén salientar que neste caso, para $\mathrm{L}=0$ a base possue 35 estados e para $\mathrm{L}=2$ e $\mathrm{L}=4$, 
70 estados devido à projeção em $\mathrm{K}=0$ e $\mathrm{K}=2$. A função de onda do modelo $\mathrm{Sp}_{11}(1, \mathrm{R})$ é dada por:

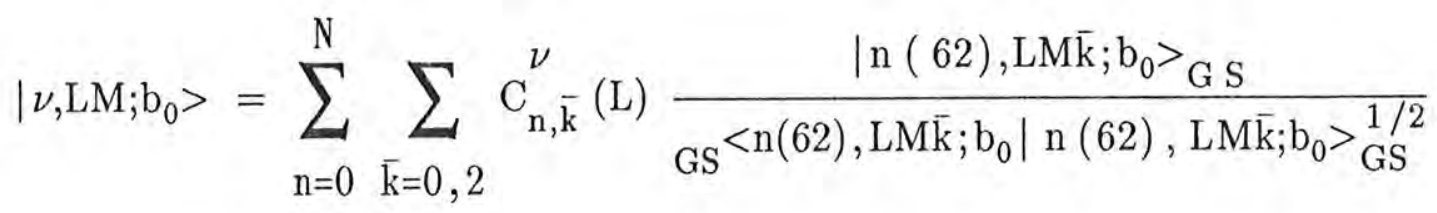

onde para $\mathrm{L}=0$ só temos $\overline{\mathrm{k}}=0$ e o comprimento do oscilador $\mathrm{b}_{0}$ foi fixado de maneira análoga à seção anterior.

$\mathrm{Na}$ figura 4.6 apresentamos os níveis de energia de excitação, obtidos pela diagonalização de $\mathrm{H}$. Observamos que os estados com $\mathrm{L}=2,4$ ocorrem em pares quase degenerados. Na figura 4.7 mostramos os coeficientes $\mathrm{C}_{\mathrm{n}, \overline{\mathrm{k}}=0}^{\nu}\left(0_{\nu}^{+}\right), \nu=1,2,3$ que obtivemos pela diagonalização da hamiltoniana. Analogamente à figura 4.2 traçamos uma linha contínua unindo os pontos relativos a cada estado $0^{+}$. Como na seção anterior notamos da figura 4.6, que quanto mais excitada é a banda os níveis de energia são mais comprimidos.

Como resultado da diagonalização da hamiltoniana, obtivemos para a banda fundamental, estados $2^{+}$e $4^{+}$que consistem em misturas de estados com $\overline{\mathrm{k}}=0$ e $\overline{\mathrm{k}}=2$ na expressão (49) e para a primeira banda excitada os estados $2^{+}$e $4^{+}$de energia mais baixa são dominadas por estados com $\overline{\mathrm{k}}=0$, e os de energia mais alta por estados com $\overline{\mathrm{k}}=2$ na expressão (49). O mesmo comportamento ocorre para a segunda banda excitada.

O comportamento que temos na figura 4.7 é análogo ao obtido na figura 4.2 , e indica que a função de onda tem componentes não desprezíveis para um grande número de estados de base do modelo. A fim de mostrarmos a relevância dos estados de base excitados, 
construimos analogamente à seção anterior, os gráficos apresentados nas figuras 4.8-4.11, que mostram a contribuição parcial dos estados de base ao momento de quadrupolo elétrico do estado $2_{1}^{+}$e ao $\mathrm{B}\left(\mathrm{E} 2,2_{\nu}^{+} \rightarrow 0_{1}^{+}\right), \nu=1,2$ e ao $\mathrm{B}\left(\mathrm{E} 2,2_{3}^{+} \rightarrow 0_{2}^{+}\right)$.

Na tabela 4.2 apresentamos os resultados obtidos para o raio quadrático médio, $<\mathrm{r}^{2}>^{1 / 2}$, e momento de quadrupolo elétrico $\mathrm{Q}_{\mathrm{el}}$, para os estados de interesse. Os resultados obtidos para o $\mathrm{B}(\mathrm{E} 2)$ são mostrados na figura 4.6. 


\section{NÍVEIS DE ENERGIA (REPRESENTAÇÃO 2P-2B)}

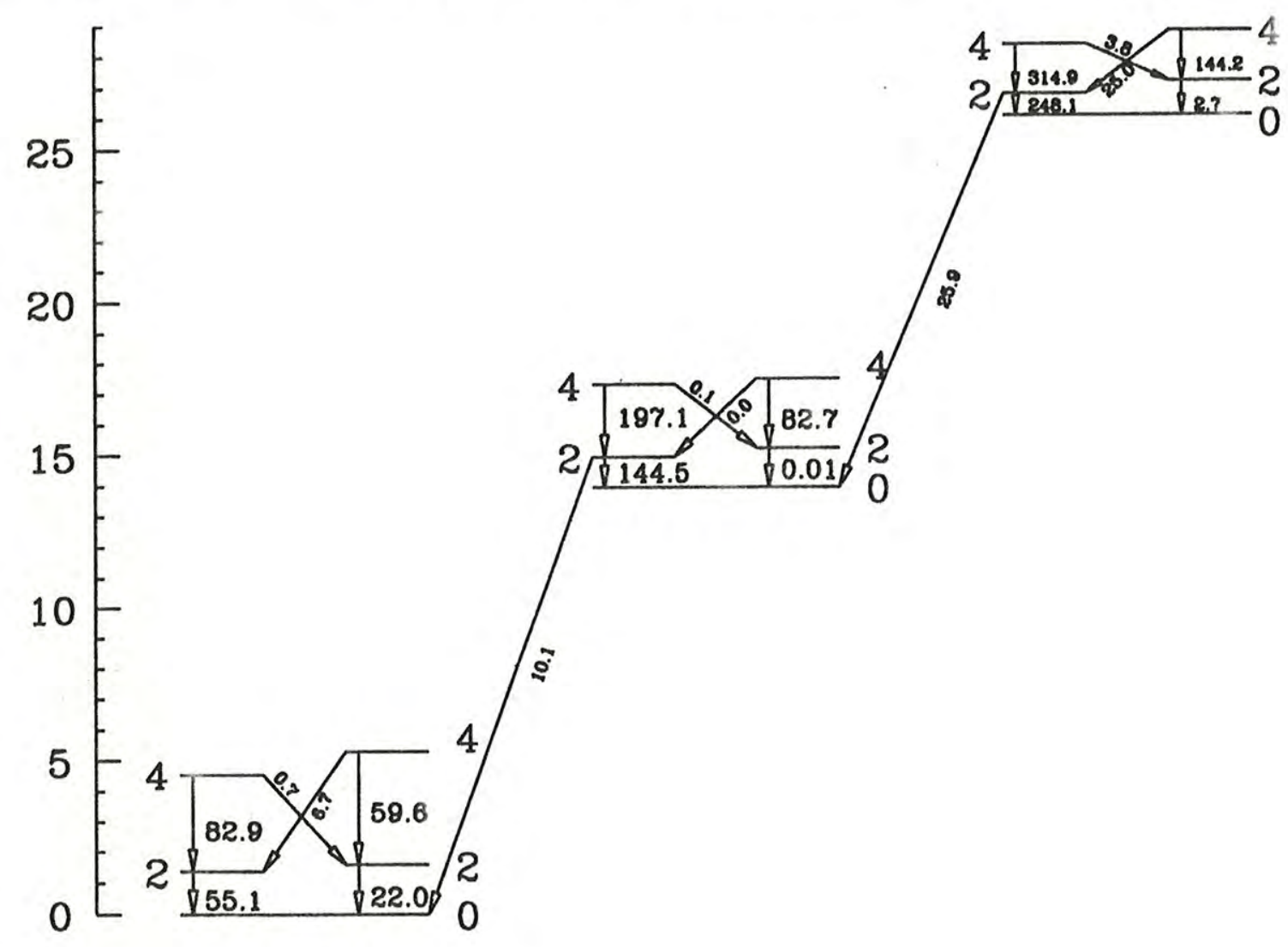

Fig. 4.6. Níveis de energia de excitação e $\mathrm{B}(\mathrm{E} 2)$ calculados no submodelo $\mathrm{Sp}_{11}(1, \mathrm{R})$ determinado pelo estado de peso mínimo $2 \mathrm{p}-2 \mathrm{~b}$. Energia do estado mais baixo $\mathrm{E}_{0}=-52.5 \mathrm{MeV}$ e $\mathrm{b}_{0}=1.58 \mathrm{fm}$. (Energias em $\mathrm{MeV}$ e $\mathrm{B}(\mathrm{E} 2)$ em $\left.\mathrm{e}^{2} \cdot \mathrm{fm}^{4}\right)$ 


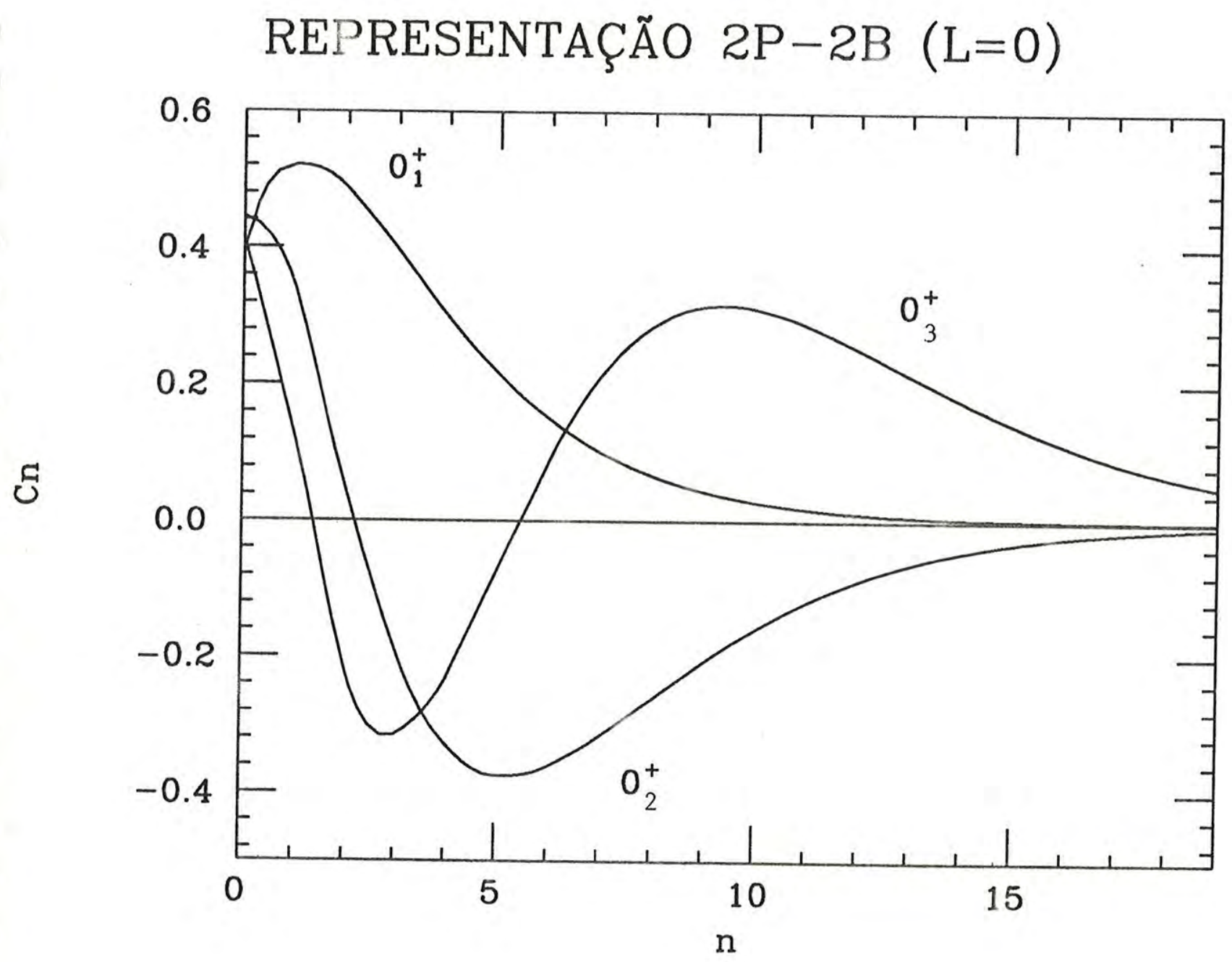

Fig. 4.7. Valores dos coeficientes $\mathrm{C}_{\mathrm{n} \overline{\mathrm{k}}=0}^{\nu}(\mathrm{L}=0)$ para os três primeiros estados $0^{+}$, como na função de $\mathrm{n}$, calculados no submodelo $\mathrm{Sp}_{\| 1}(1, \mathrm{R})$ determinado pelo estado de peso mínimo $2 \mathrm{p}-2 \mathrm{~b}$. 


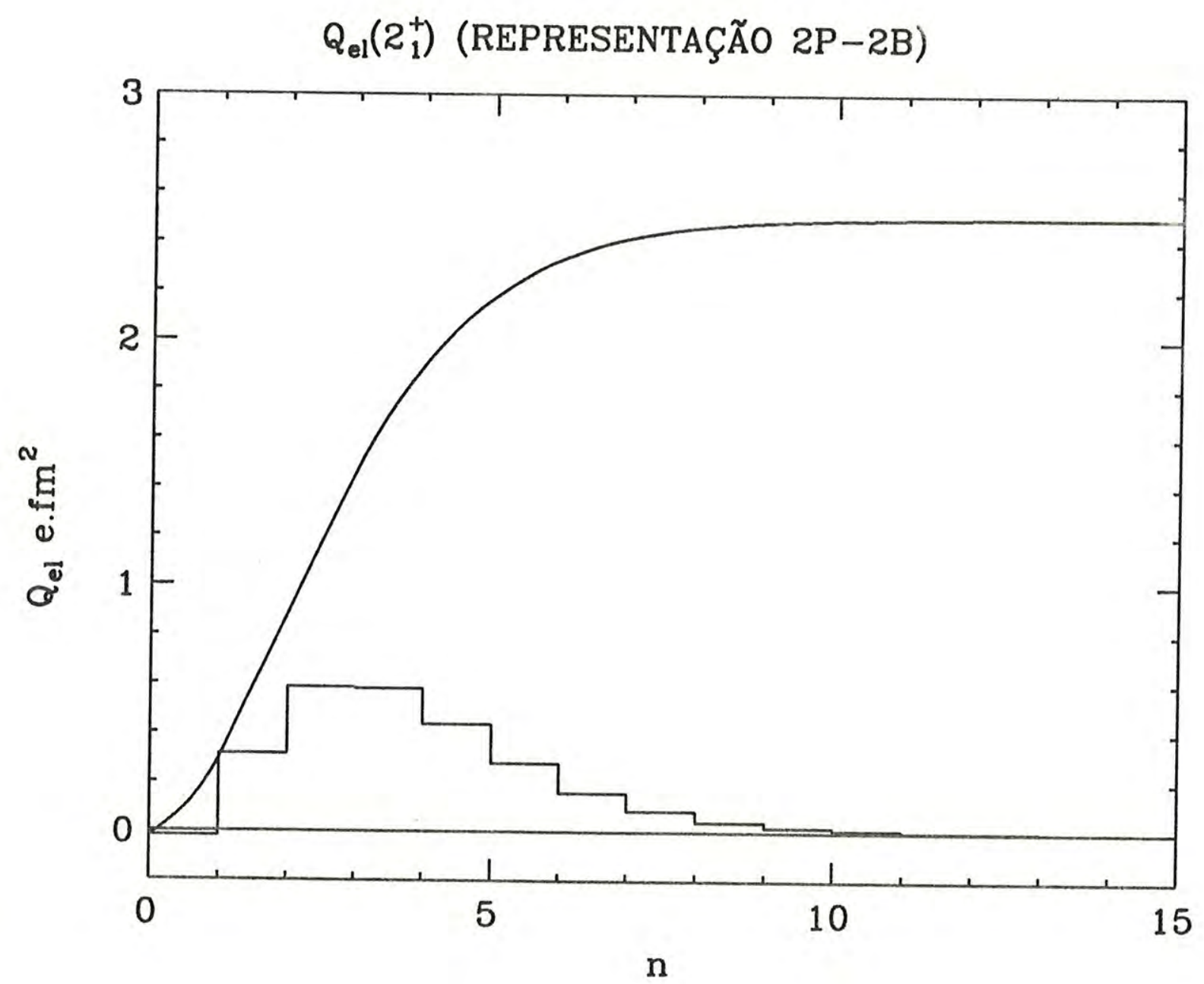

Fig. 4.8. Contribuição parcial ao $\mathrm{Q}_{\mathrm{el}}\left(2_{1}^{+}\right)$incluindo de maneira sistemática,na função de onda dada pela eq.(4.49), os estados de base do modelo $\mathrm{Sp}_{\| 1}(1, \mathrm{R})$. Para cada $n$ sempre consideramos os estados com $\bar{k}=0$ e $\bar{k}=2$ correspondentes. $\mathrm{O}$ valor de $\mathrm{Q}_{\mathrm{el}}\left(2_{1}^{+}\right)$no ponto $\mathrm{n}$,é obtido considerando-se na expansão (49), a contribuição de todos os termos até $n$, inclusive. 


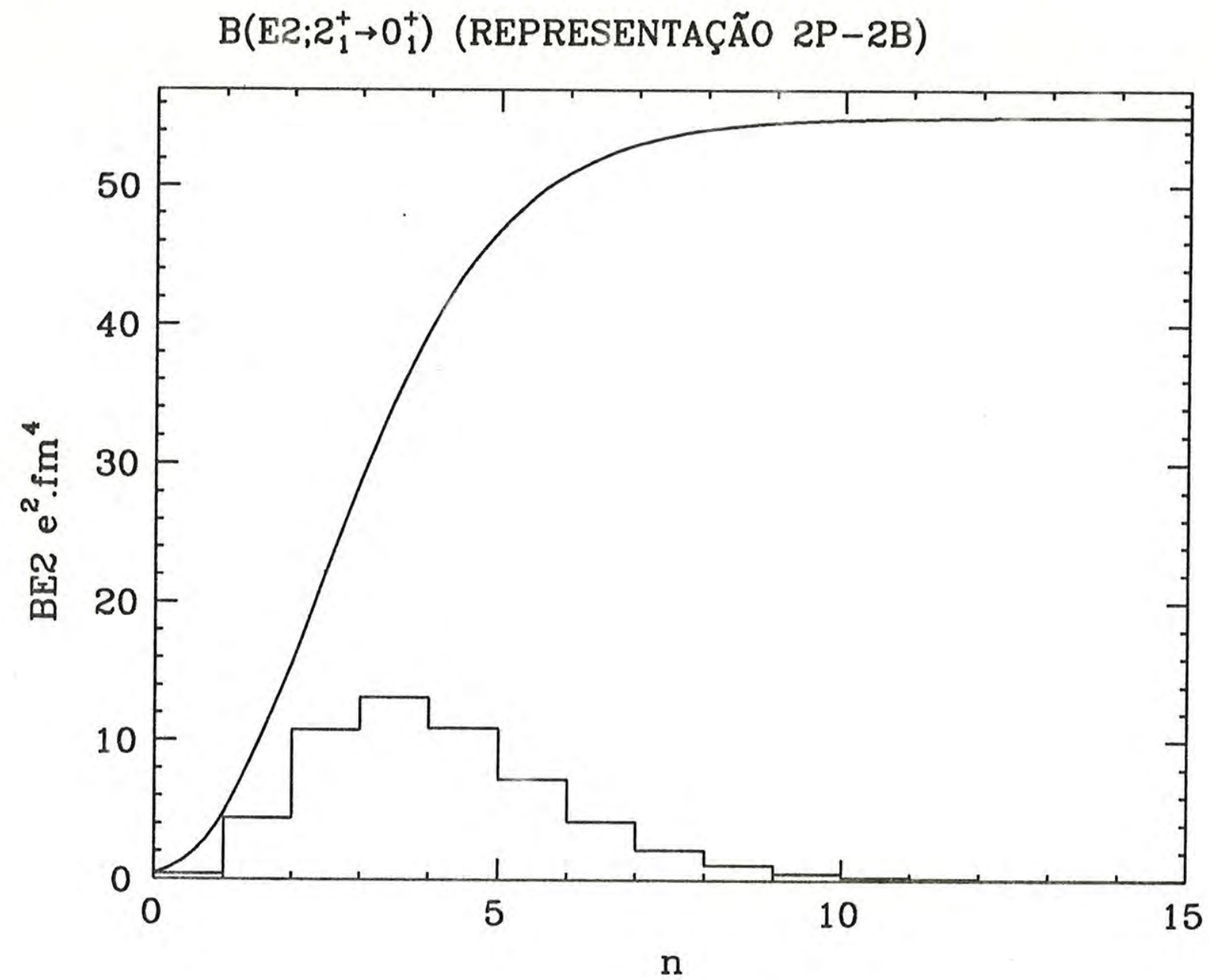

Fig. 4.9. Contribuição parcial ao $\mathrm{B}\left(\mathrm{E} 2,2_{1}^{+} \rightarrow 0_{1}^{+}\right)$, incluindo de maneira sistemática, na eq.(4.49), os estados de base do modelo $\mathrm{Sp}_{11}(1, \mathrm{R})$. Para cada $\mathrm{n}$ sempre consideramos os estados com $\overline{\mathrm{k}}=0$ e $\overline{\mathrm{k}}=2$ correspondentes. O valor do $\mathrm{B}(\mathrm{E} 2)$ no ponto $\mathrm{n}$,é obtido considerando-se na expansão (49), a contribuição de todos os termos até $\mathrm{n}$, inclusive. 


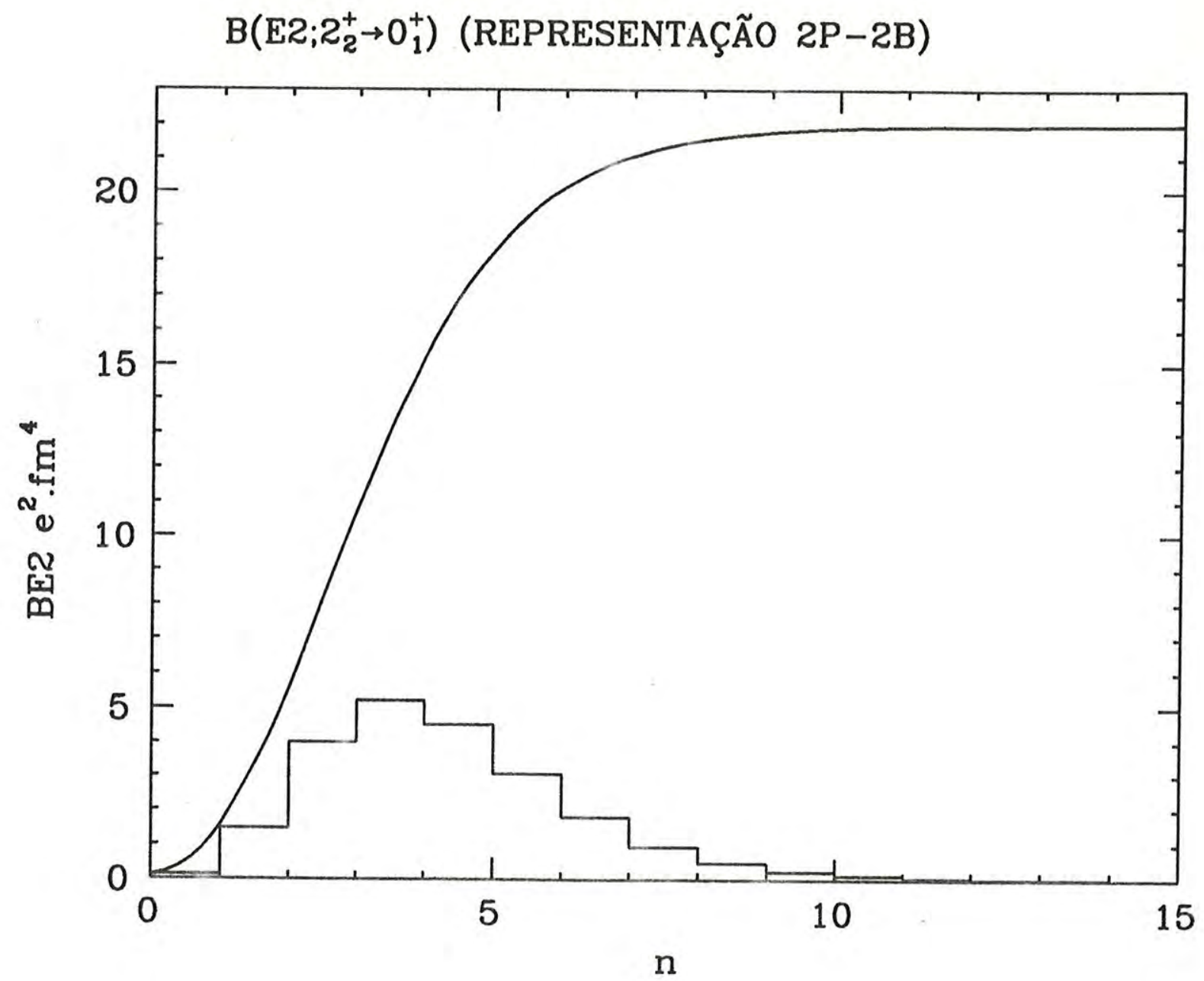

Fig. 4.10. Contribuição parcial ao $\mathrm{B}\left(\mathrm{E} 2,2_{2}^{+} \rightarrow 0_{1}^{+}\right)$incluindo de maneira sistemática,na eq.(4.49), os estados de base do modelo $\mathrm{Sp}_{\| 1}(1, \mathrm{R})$. Para cada $\mathrm{n}$ sempre consideramos os estados com $\overline{\mathrm{k}}=0$ e $\overline{\mathrm{k}}=2$ correspondentes. $\mathrm{O}$ valor do $\mathrm{B}(\mathrm{E} 2)$ no ponto $\mathrm{n}$,é obtido considerando-se na expansão (49), a contribuição de todos os termos até $\mathrm{n}$, inclusive. 


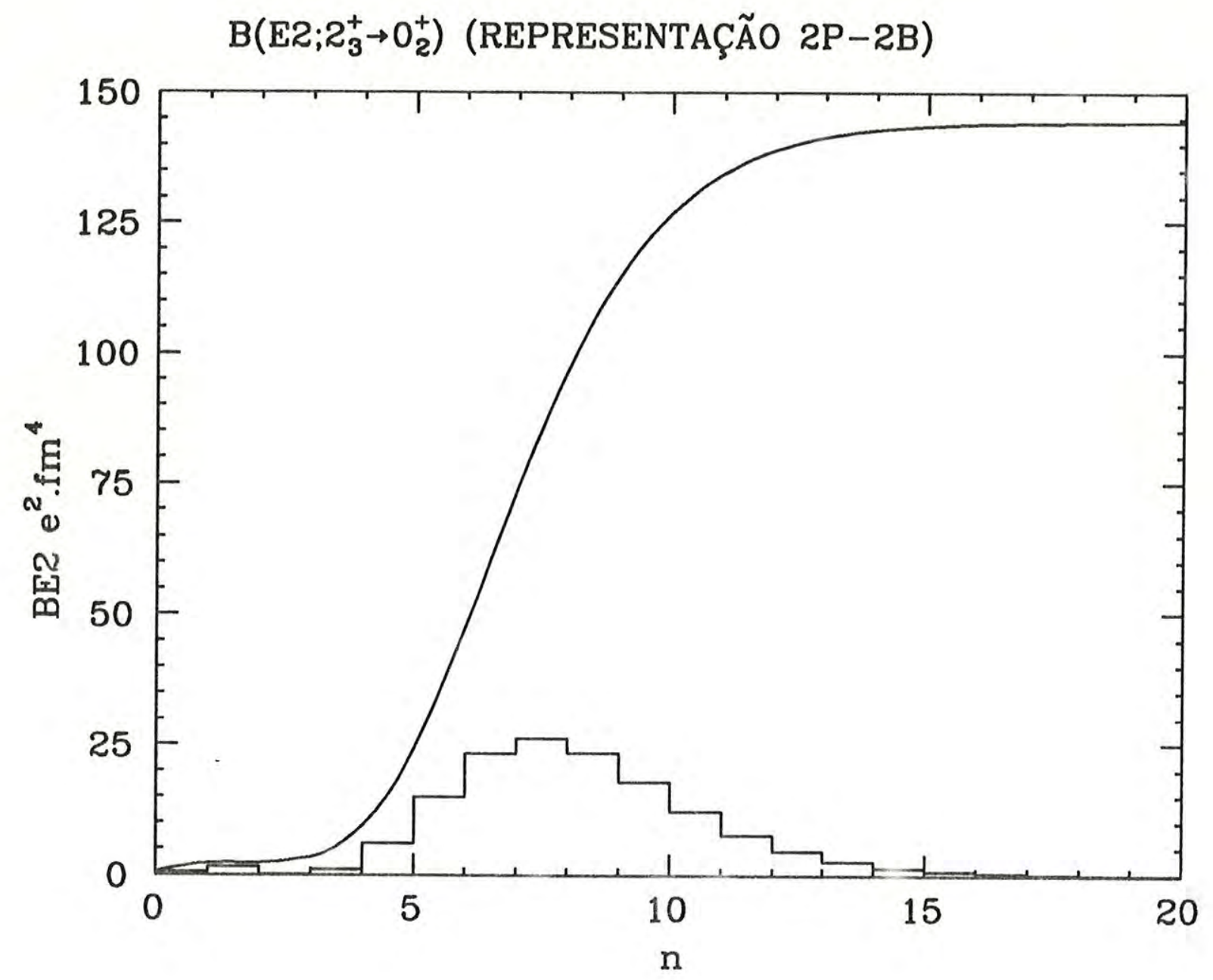

Fig. 4.11. Contribuição parcial ao $\mathrm{B}\left(\mathrm{E} 2,2_{3}^{+} \rightarrow 0_{2}^{+}\right)$, incluindo de maneira sistemática, na eq.(4.49), os estados de base do modelo $\mathrm{Sp}_{\| 1}(1, \mathrm{R})$. Para cada $\mathrm{n}$ sempre consideramos os estados com $\overline{\mathrm{k}}=0$ e $\overline{\mathrm{k}}=2$ correspondentes. $\mathrm{O}$ valor do $\mathrm{B}(\mathrm{E} 2)$ no ponto $\mathrm{n}$,é obtido considerando-se na expansão (49), a contribuição de todos os termos até $\mathrm{n}$, inclusive. 


\begin{tabular}{|c|c|c|}
\hline $\mathrm{L}_{\nu}^{\pi}$ & $<\mathrm{r}^{2}>^{1 / 2}$ & $\mathrm{Q}_{\mathrm{el}}$ \\
\hline $0_{1}^{+}$ & 2.99 & - \\
\hline $2_{1}^{+}$ & 2.99 & -2.50 \\
\hline $2_{2}^{+}$ & 2.98 & +2.68 \\
\hline $4_{1}^{+}$ & 2.98 & -12.87 \\
\hline $4_{2}^{+}$ & 2.95 & -17.83 \\
\hline $0_{2}^{+}$ & 3.30 & $\longrightarrow$ \\
\hline $2_{3}^{+}$ & 3.30 & -23.76 \\
\hline $2_{4}^{+}$ & 3.30 & 24.03 \\
\hline $4_{3}^{+}$ & 3.26 & -29.22 \\
\hline $4_{4}^{+}$ & 3.26 & -12.80 \\
\hline $0_{3}^{+}$ & 3.63 & - \\
\hline $2_{5}^{+}$ & 3.61 & -31.92 \\
\hline $2_{6}^{+}$ & 3.63 & 32.26 \\
\hline $4_{5}^{+}$ & 3.57 & -39.11 \\
\hline $4_{6}^{+}$ & 3.61 & -16.55 \\
\hline
\end{tabular}

Tab. 4.2. Propriedades geométricas dos estados calculados no modelo $\operatorname{Sp}_{11}(1, R)$, determinado pelo estado de peso mínimo $2 \mathrm{p}-2 \mathrm{~b}$, e que associamos a estados excitados do ${ }^{12} \mathrm{C} \cdot\left(<\mathrm{r}^{2}\right\rangle^{1 / 2}$ em fm, $\mathrm{Q}_{\mathrm{el}}$ em e.fm $\left.{ }^{2}\right)$. 


\subsubsection{Submodelo $\mathrm{Sp}_{11}(1, \mathrm{R})$ Determinado pelo Estado de Peso Mínimo $4 \mathrm{p}-4 \mathrm{~b}$}

Neste caso, a base de estados do modelo $\mathrm{Sp}_{11}(1, \mathrm{R})$ é dada pelos estados (21) axialmente simétricos. Os níveis de energia e funções de onda são obtidos pela diagonalização da hamiltoniana na base de estados (21). Analogamente à seção 4.5.1, uma base com $N=34$ foi suficiente para obtermos a convergência com $\varepsilon=0.0001$. A função de onda neste caso é dada por:

$$
\left|\nu, \mathrm{LM} ; \mathrm{b}_{0}\right\rangle=\sum_{\mathrm{n}=0}^{\mathrm{N}} \mathrm{C}_{\mathrm{n}}^{\nu}(\mathrm{L}) \frac{\left|\mathrm{n}(120), \mathrm{LM} 0 ; \mathrm{b}_{0}\right\rangle}{<\mathrm{n}(120), \mathrm{LM} 0 ; \mathrm{b}_{0} \mid \mathrm{n}(120), \mathrm{LM} 0 ; \mathrm{b}_{0}>^{1 / 2}} .
$$

Na figura 4.12 apresentamos os resultados obtidos para os níveis de energia de excitação. Na figura 4.13 mostramos os coeficientes $\mathrm{C}_{\mathrm{n}}^{\nu}\left(0_{\nu}^{+}\right), \nu=1,2,3$, da função de onda (50) e traçamos uma linha contínua unindo os pontos relativos a cada estado.

Notemos na figura 4.12 que analogamente ao caso op-ob, os níveis de energia se comprimem quando consideramos bandas mais excitadas e os $\mathrm{B}(\mathrm{E} 2)$ intra-banda aumentam. Comparando com a figura 4.1, notamos que o espectro neste caso é bem mais comprido e os $\mathrm{B}(\mathrm{E} 2)$ intra-banda bem maiores, o que sugere uma maior deformação. A figura 4.13 nos mostra que a função de onda tem componentes não desprezíveis para vários estados de base sendo este comportamento também encontrado nas figuras 4.2 e 4.7. No presente caso, os estados de base excitados têm um papel ainda mais relevante. Por exemplo, para o estado fundamental os estados dominantes têm $n=4,5$ e 6 .

Para mostrarmos a importância dos estados de base excitados, construímos as figuras 4.14-4.16 de maneira análoga à figura 4.3. Nessas figuras mostramos a contribuição parcial dos estados de base ao momento de quadrupolo elétrico $2_{1}^{+}$e ao $\mathrm{B}\left(\mathrm{E} 2,2_{\nu}^{+} \rightarrow 0_{\nu}^{+}\right)$, $\nu=1,2$. Das figuras 4.14-4.16 fica evidente a importância das configurações excitadas.

$\mathrm{Na}$ tabela 4.3 apresentamos os resultados obtidos para o $<\mathrm{r}^{2}>^{1 / 2}$ e $\mathrm{Q}_{\mathrm{el}}$ para os estados de interesse. Os resultados obtidos para o $\mathrm{B}(\mathrm{E} 2)$ mostramos na figura 4.12. 


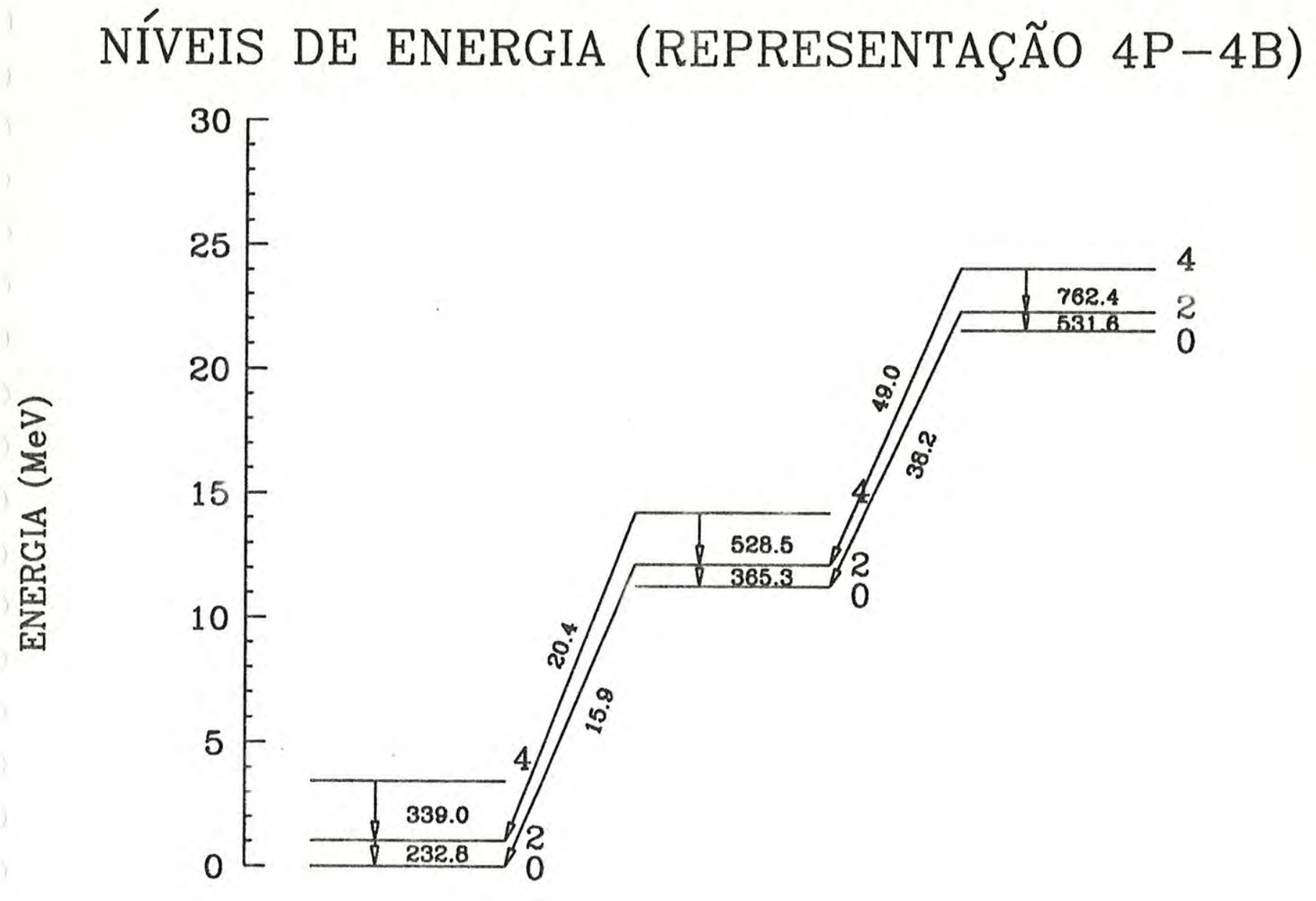

Fig. 4.12. Níveis de energia de excitação e $\mathrm{B}(\mathrm{E} 2)$ calculados no submodelo $\mathrm{Sp}_{\|}(1, \mathrm{R})$ determinado pelo estado de peso mínimo $4 \mathrm{p}-4 \mathrm{~b}$. Energia do estado mais baixo $\mathrm{E}_{0}=-64.3 \mathrm{MeV}$ e $\mathrm{b}_{0}=1.50 \mathrm{fm}$. (Energias em $\mathrm{MeV}$ e B(E2) em $\left.\mathrm{e}^{2} \cdot \mathrm{fm}^{4}\right)$ 


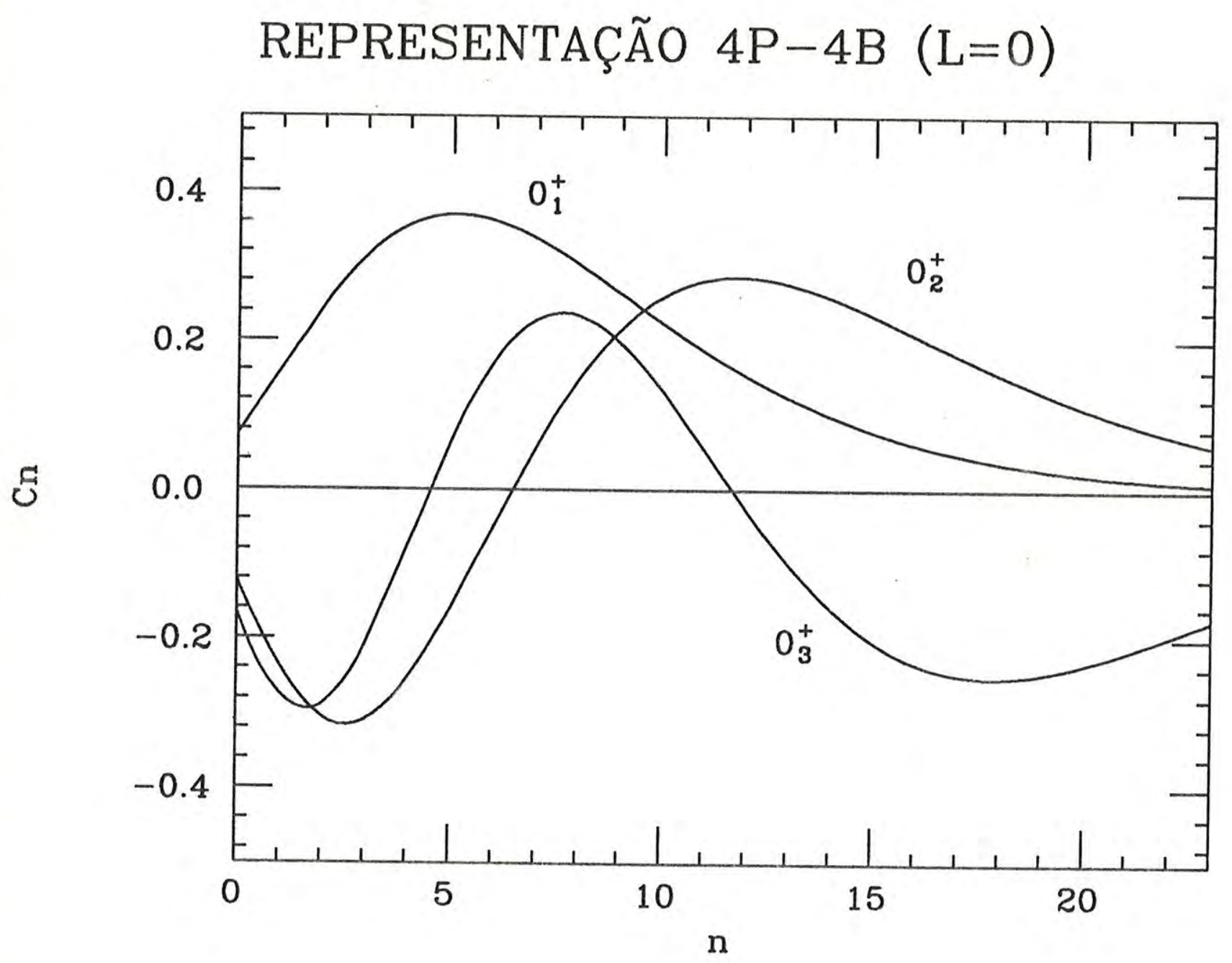

Fig. 4.13. Valores dos coeficientes $\mathrm{C}_{\mathrm{n}}^{\nu}\left(\mathrm{L}=0^{+}\right)$para os irês primeiros estados $0^{+}$, como na função de $\mathrm{n}$, calculados no modelo $\mathrm{Sp}_{11}(1, \mathrm{R})$ associado ao estado de peso mínimo $4 \mathrm{p}-4 \mathrm{~b}$. 


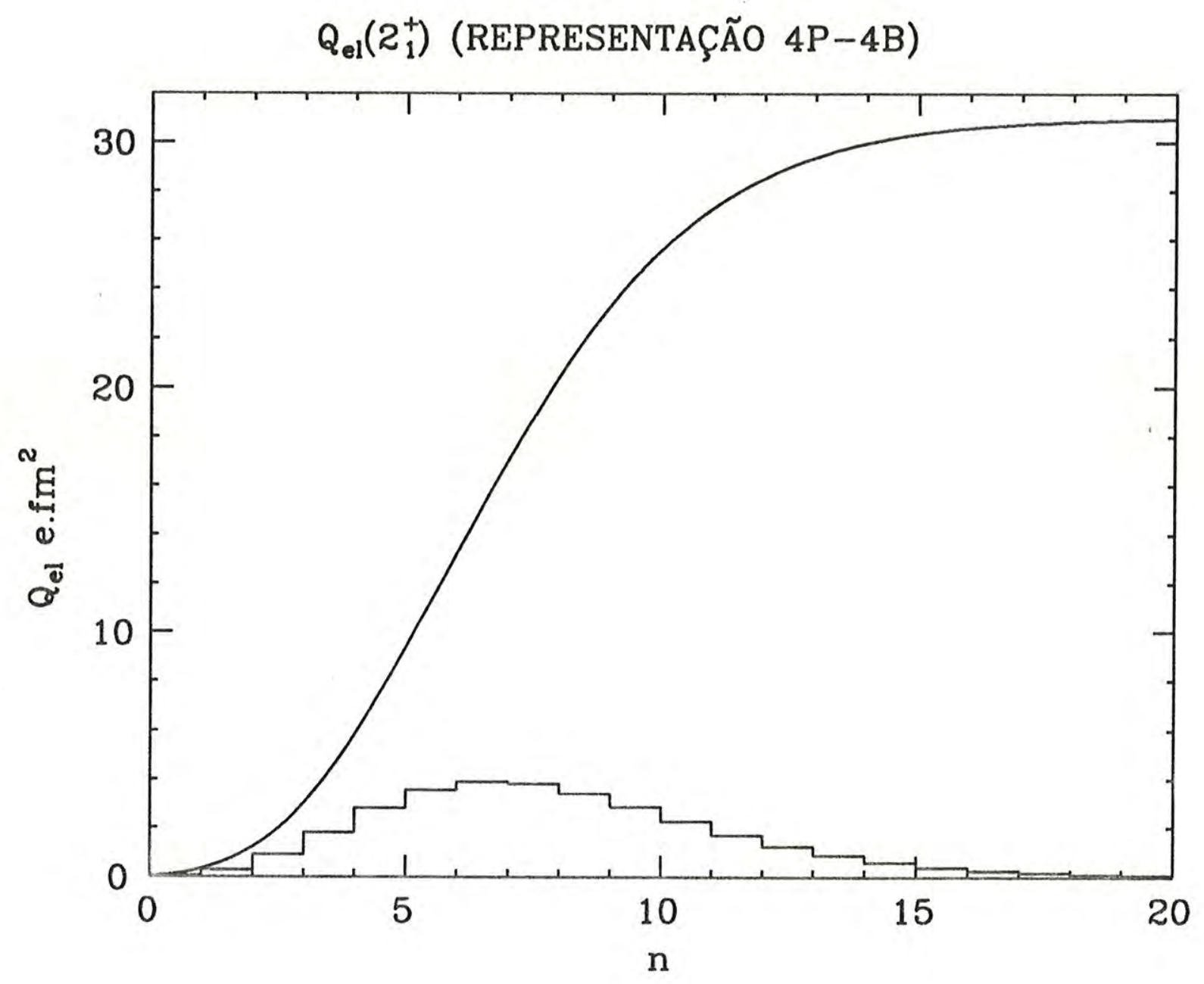

Fig. 4.14. Contribuição parcial ao $\mathrm{Q}_{\mathrm{el}}\left(2_{1}^{+}\right)$devido à inclusão sistemática, na função de onda dada pela eq.(4.50), dos estados de base do modelo $\operatorname{Sp}_{11}(1, R)$. O valor do $\mathrm{Q}_{\mathrm{el}}\left(2_{1}^{+}\right)$no ponto $\mathrm{n}$, é obtido considerando-se na expansão (50), a contribuição de todos os termos até $n$, inclusive. 


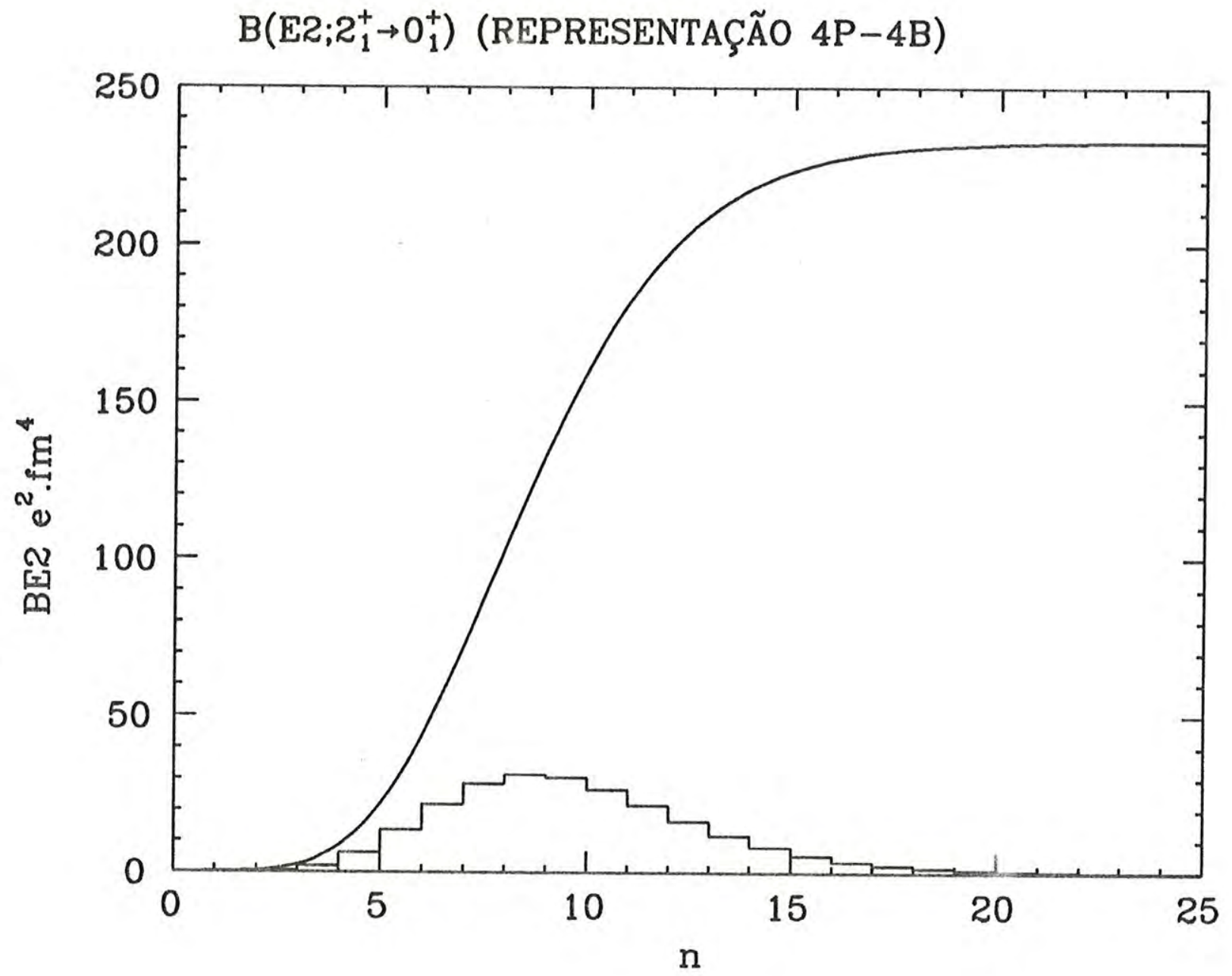

Fig. 4.15. Contribuição parcial ao $\mathrm{B}\left(\mathrm{E} 2,2_{1}^{+} \rightarrow 0_{1}^{+}\right)$devido à inclusão sistemática, na eq.(4.50), dos estados de base do modelo $\mathrm{Sp}_{11}(1, \mathrm{R})$. O valor do $\mathrm{B}(\mathrm{E} 2)$ no ponto $n$,é obtido considerando-se na expansão (50), a contribuição de todos os termos até $\mathrm{n}$, inclusive. 


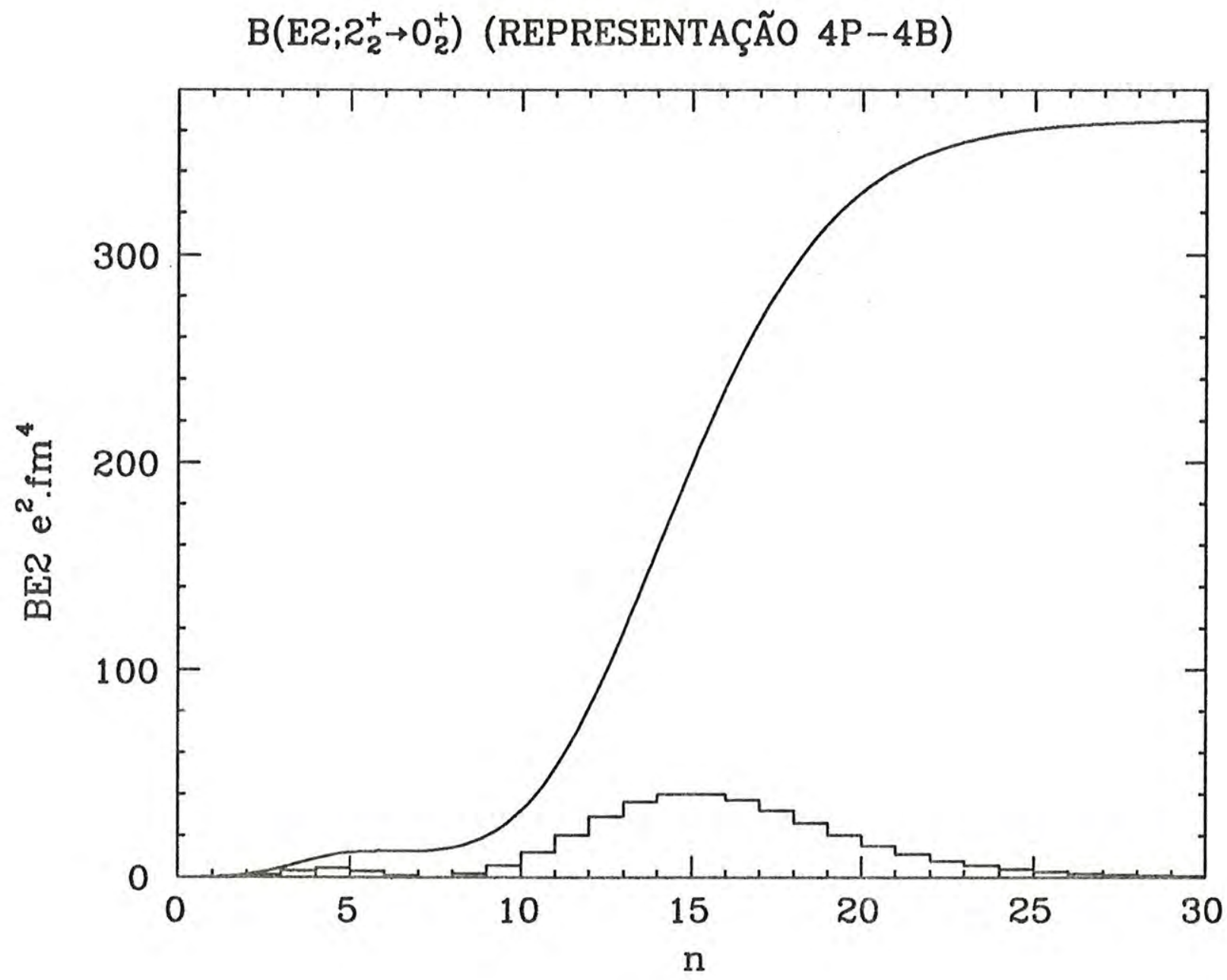

Fig. 4.16. Contribuição parcial ao $\mathrm{B}\left(\mathrm{E} 2,2_{2}^{+} \rightarrow 0_{2}^{+}\right)$devido à inclusão sistemática, na eq.(4.50), dos estados de base do modelo $\mathrm{Sp}_{11}(1, \mathrm{R})$. O valor do $\mathrm{B}(\mathrm{E} 2)$ no ponto $n$,é obtido considerando-se na expansão (50), a contribuição de todos os termos até $\mathrm{n}$, inclusive. 


\begin{tabular}{rrr}
\hline $\mathrm{L}_{\nu}^{\pi}$ & $<\mathrm{r}^{2}>^{1 / 2}$ & $\mathrm{Q}_{\mathrm{el}}$ \\
\hline $0_{1}^{+}$ & 3.41 & - \\
$2_{1}^{+}$ & 3.41 & -31.00 \\
$4_{1}^{+}$ & 3.43 & -40.03 \\
$0_{2}^{+}$ & 3.73 & - \\
$2_{2}^{+}$ & 3.73 & -38.81 \\
$4_{2}^{+}$ & 3.75 & -49.91 \\
$0_{3}^{+}$ & 4.03 & - \\
$2_{3}^{+}$ & 4.03 & -46.76 \\
$4_{3}^{+}$ & 4.04 & -59.77 \\
\hline
\end{tabular}

Tab. 4.3. Propriedades geométricas dos estados calculados no modelo $\mathrm{Sp}_{\| 1}(1, \mathrm{R})$ associado ao estado de peso mínimo $4 \mathrm{p}-4 \mathrm{~b} \cdot\left(<\mathrm{r}^{2}>^{1 / 2}\right.$ em fm, $\mathrm{Q}_{\mathrm{el}}$ $\left.\mathrm{em} \cdot \mathrm{fm}^{2}\right)$. 


\section{Cálculo $\operatorname{Sp}(1, \mathrm{R})$ Em Uma Base Deformada}

\subsection{Introdução}

No capítulo anterior procuramos descrever o espectro de baixa energia do ${ }^{12} \mathrm{C}$, utilizando o modelo $\mathrm{Sp}(1, \mathrm{R})$ construído a partir de funções de onda do oscilador harmônico esférico. A análise dos resultados obtidos nos mostra de maneira inequívoca a importância das configurações excitadas na descrição dos observáveis de interesse, vimos inclusive que em muitos casos as configurações excitadas dominavam.

Nas próximas seções analisaremos o cálculo $\mathrm{Sp}(1, \mathrm{R})$ realizado no último capítulo, onde agora utilizaremos uma base otimizada equivalente ${ }^{(9)}$, isto é, uma base construída a partir de funções de onda do oscilador harmônico deformado. Mostraremos que a necessidade de considerarmos um grande número de estados no cálculo $\mathrm{Sp}(1, \mathrm{R})$ usando uma base esférica se deve à falta de otimização da base.

Analisaremos a relação entre o modelo $\mathrm{Sp}(1, \mathrm{R})$ e cálculos do tipo projeção antes da variação (PAV) de um oscilador harmônico deformado, mostrando que em várias situações o PAV, em boa aproximação, é equivalente ao modelo $\mathrm{Sp}(1, \mathrm{R})$.

Organizamos este capítulo da seguinte maneira:

$\mathrm{Na}$ seção 5.2 consideraremos o modelo $\mathrm{Sp}_{\perp}(1, \mathrm{R})$ em uma base deformada que, como vimos no capítulo anterior, é associado ao estado de peso mínimo op-ob e na seção 5.3 consideraremos o modelo $\mathrm{Sp}_{11}(1, \mathrm{R})$ em uma base deformada que, de acordo com 0 último capítulo, associamos aos estados de peso mínimo $2 \mathrm{p}-2 \mathrm{~b}$ e $4 \mathrm{p}-4 \mathrm{~b}$. 


\subsection{Modelo $\mathrm{Sp}_{\perp}(1, \mathrm{R})$ Deformado}

A partir da base de estados (4.3), constrúda em termos de funções de onda do oscilador harmônico esférico, podemos obter bases equivalentes através de transformações unitárias pertencentes ao grupo $\mathrm{Sp}_{\perp}(1, \mathrm{R})$. A fim de obtermos uma base deformada para 0 modelo $S p_{\perp}(1, R)$, vamos utilizar a transformação unitária que corresponde à transformações de escala no plano perpendicular ao eixo de simetria axial, gerada pelo operador abaixo:

$$
S_{\perp}=i\left[A_{\perp}^{+}\left(b_{0}\right)-A_{\perp}\left(b_{0}\right)\right]
$$

Para obtermos a ação do operador acima lembramos que, por definição o operador gerador de transformação de escala na direção $\mu$, é dado por $\mathrm{S}_{\mu}=\mathrm{i}\left[\mathrm{A}_{\mu \mu}^{+}-\mathrm{A}_{\mu \mu}\right]$ onde é fácil mostrar que:

$$
\mathrm{e}^{-\mathrm{i} \theta \mathrm{S}_{\mu \mu}}\left\{\begin{array}{c}
\rho_{\mu}(\mathrm{k}) \\
\pi_{\mu}(\mathrm{k})
\end{array}\right\} \text { e } \mathrm{e}^{\mathrm{i} \theta \mathrm{S}}=\left\{\begin{array}{l}
\mathrm{e}^{-\theta} \rho_{\mu}(\mathrm{k}) \\
\mathrm{e}^{\theta} \pi_{\mu}(\mathrm{k})
\end{array}\right\}
$$

e segue imediatamente das relações acima que

$$
\mathrm{e}^{-\mathrm{i} \theta \mathrm{S}_{\mu \mu}} \mathrm{a}_{\mu ; \mathrm{k}}^{+}\left(\mathrm{b}_{0}\right) \mathrm{e}^{\mathrm{i} \theta \mathrm{S}} \mu \mu=\mathrm{a}_{\mu ; \mathrm{k}}^{+}\left(\mathrm{b}_{0} \mathrm{e}^{\theta}\right)
$$

A partir das relações (1) e (2) segue que a transformação de escala no plano perpendicular ao eixo de simetria, gerada por $\mathrm{S}_{\perp}$, aplicada ao estado (4.3):

$$
\left|n ; b_{0} b_{\perp}>=e^{-i \ln \left(b_{\perp} / b_{0}\right) S_{\perp}}\right| n ; b_{0}>
$$


e aos geradores da álgebra $\mathrm{Sp}_{\perp}(1, \mathrm{R}),(4.1)$ :

$$
\left\{\begin{array}{l}
A_{\perp}^{+}\left(b_{\perp}\right) \\
A_{\perp}\left(b_{\perp}\right) \\
A_{0_{\perp}}\left(b_{\perp}\right)
\end{array}\right\}=e^{-i \ln \left(b_{\perp} / b_{0}\right) S_{\perp}}\left\{\begin{array}{c}
A_{\perp}^{+}\left(b_{0}\right) \\
A_{\perp}\left(b_{0}\right) \\
A_{0_{\perp}}\left(b_{0}\right)
\end{array}\right\} e^{i \ln \left(b_{\perp} / b_{0}\right) S_{\perp}}
$$

resulta em:

$$
\begin{aligned}
& \left|\mathrm{n} ; \mathrm{b}_{0} \mathrm{~b}_{\perp}\right\rangle=\left[\mathrm{A}_{\perp}^{+}\left(\mathrm{b}_{\perp}\right)\right]^{\mathrm{n}} \mid 0 ; \mathrm{b}_{0} \mathrm{~b}_{\perp}>\left[\frac{\Gamma\left(2 \mathrm{k}_{\perp}\right)}{\Gamma(\mathrm{n}+1) \Gamma\left(\mathrm{n}+2 \mathrm{k}_{\perp}\right)}\right]^{1 / 2} \\
& A_{\perp}^{+}\left(b_{\perp}\right)=A_{22}^{+}\left(b_{\perp}\right)+A_{33}^{+}\left(b_{\perp}\right)=\frac{1}{2} \sum_{k=1}^{A-1} a_{2 ; k}^{+}\left(b_{\perp}\right) a_{2 ; k}^{+}\left(b_{\perp}\right)+ \\
& +\frac{1}{2} \sum_{k=1}^{A-1} a_{3 ; k}^{+}\left(b_{\perp}\right) a_{3 ; k}^{+}\left(b_{\perp}\right)+ \\
& A_{\perp}\left(b_{\perp}\right)=\left[A_{\perp}^{+}\left(b_{\perp}\right)\right]^{+} \\
& \mathrm{A}_{0_{\perp}}\left(\mathrm{b}_{\perp}\right)=\frac{1}{2} \mathrm{C}_{22}\left(\mathrm{~b}_{\perp}\right)+\frac{1}{2} \mathrm{C}_{33}\left(\mathrm{~b}_{\perp}\right)=\frac{1}{4} \sum_{\mathrm{k}=1}^{\mathrm{A}-1} \mathrm{a}_{2 ; \mathrm{k}}^{+}\left(\mathrm{b}_{\perp}\right) \mathrm{a}_{2 ; \mathrm{k}}\left(\mathrm{b}_{\perp}\right)+ \\
& +a_{2 ; k}\left(b_{\perp}\right) a_{2 ; k}^{+}\left(b_{\perp}\right)+\frac{1}{4} \sum_{k=1}^{A-1} a_{3 ; k}^{+}\left(b_{\perp}\right) a_{3 ; k}\left(b_{\perp}\right)+a_{3 ; k}\left(b_{\perp}\right) a_{3 ; k}^{+}\left(b_{\perp}\right) .
\end{aligned}
$$

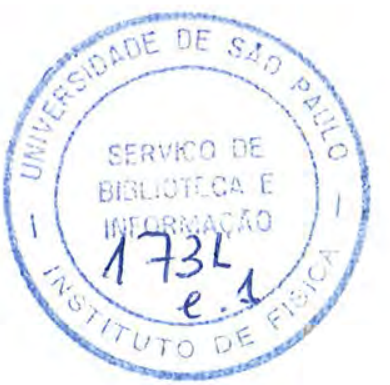


Os operadores $A_{\perp}^{+}\left(b_{\perp}\right), A_{\perp}\left(b_{\perp}\right)$ e $A_{0_{\perp}}\left(b_{\perp}\right)$ formam uma base equivalente da álgebra $\mathrm{sp}_{\perp}(1, \mathrm{R})$ dada em (4.1). Do mesmo modo, os estados $\left|\mathrm{n} ; \mathrm{b}_{0} \mathrm{~b}_{\perp}\right\rangle$ formam uma base de estados equivalentes da representação irredutível $\mathrm{k}_{\perp}$ de $\mathrm{Sp}_{\perp}(1, \mathrm{R})$. O efeito da transformação de escala em $\left|n ; b_{0}\right\rangle$ é substituir as funções de onda do oscilador harmônico esférico, onde $b_{x}=b_{y}=b_{z}=b_{0}$ pelas funções de onda do oscilador harmônico deformado onde $b_{x}=b_{y}=b_{\perp}$ e $b_{z}=b_{0}$.

Os estados $\left|\mathrm{n} ; \mathrm{b}_{0} \mathrm{~b}_{\perp}\right\rangle$ possuem as mesmas propriedades e significado físico dos estados $\left|n ; b_{0}\right\rangle$ e somente agora nos referimos à base equivalente de operadores obtida pela transformação de escala gerado por $S_{\perp}$. Analogamente ao caso esférico, os estados $\mid \mathrm{n} ; \mathrm{b}_{0} \mathrm{~b}_{\perp}>$ podem ser interpretados como estados de $\mathrm{n}$ fonons deformados $(9)$.

Os coeficientes da expansão de $\left|n ; b_{0} b_{\perp}\right\rangle$ nos estados esféricos $\left|n ; b_{0}\right\rangle$ :

$$
\left|n ; b_{0} b_{\perp}\right\rangle=\sum_{n^{\prime}=0}^{\infty} \mid n^{\prime} ; b_{0}>U_{n^{\prime} n}
$$

podem ser calculados explicitamente ${ }^{(9)}$ :

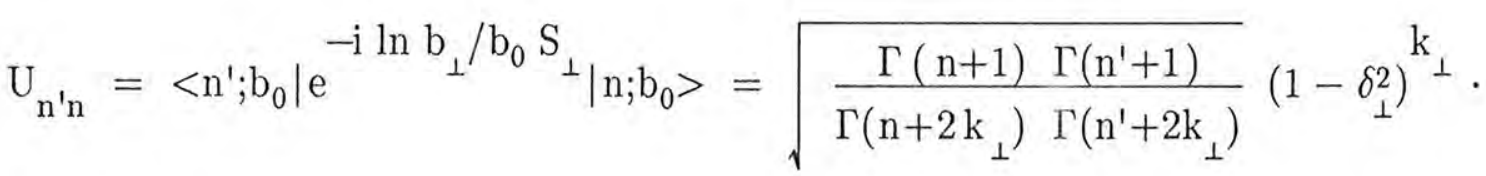

$$
\begin{aligned}
& \cdot \sum_{r=0}^{\min \left\{n, n^{\prime}\right\}} \frac{\Gamma\left(2 k_{\perp}+n+n^{\prime}-r\right)(-1)^{n-r}}{\Gamma(n-r+1) \Gamma\left(n^{\prime}-r+1\right) \Gamma(r+1)} \delta_{\perp}^{n+n^{\prime}-2 r}
\end{aligned}
$$

onde $\min \left\{n, n^{\prime}\right\}$ significa o menor entre os números $n$ e $n^{\prime}$ e o parâmetro da deformação $\delta_{\perp}$ é dado por: 


$$
\delta_{\perp}=\frac{b_{\perp}^{2}-b_{0}^{2}}{b_{\perp}^{2}+b_{0}^{2}}
$$

Aplicando o operador projeção em momento angular à expressão (5) obtemos a relação entre as bases equivalentes do modelo $\operatorname{Sp}_{\perp}(1, \mathrm{R})$ :

$$
\left|\mathrm{n}, \mathrm{LM} 0 ; \mathrm{b}_{0} \mathrm{~b}_{\perp}>=\sum_{\mathrm{n}^{\prime}=0}^{\infty}\right| \mathrm{n}^{\prime}, \mathrm{LM} 0 ; \mathrm{b}_{0}>\mathrm{U}_{\mathrm{n}^{\prime} \mathrm{n}} .
$$

Os estados acima não são ortogonais e para $\mathrm{L}>\mu$ eles são linearmente dependentes (L.D.), onde o número de estados L.D. é igual a $\mathrm{n}_{\mathrm{L}}=\frac{\mathrm{L}-\mu}{2}$. Recordemos que estamos supondo o estado $|0\rangle$ com rótulos de Elliott $(0 \mu)$.

A base de estados equivalente $\left|\mathrm{n}, \mathrm{LMO} ; \mathrm{b}_{0} \mathrm{~b}_{\perp}\right\rangle$ depende do parâmetro $\mathrm{b}_{\perp}$, que corresponde ao comprimento do oscilador no plano perpendicular ao eixo de simetria axial.

Nós fixamos $b_{\perp}$ utilizando o método da projeção antes da variação PAV, impondo que o valor esperado da hamiltoniana no estado do vácuo do fonon transversal deformado, projetado em momento angular $\mathrm{L}$, seja um mínimo como função de $b_{\perp}$ :

$$
\frac{\partial}{\partial \mathrm{b}_{\perp}} \frac{<0, \mathrm{LM} 0 ; \mathrm{b}_{0} \mathrm{~b}_{\perp}|\mathrm{H}| 0, \mathrm{LM} 0 ; \mathrm{b}_{0} \mathrm{~b}_{\perp}>}{<0, \mathrm{LM} 0 ; \mathrm{b}_{0} \mathrm{~b}_{\perp} \mid 0, \mathrm{LM} 0 ; \mathrm{b}_{0} \mathrm{~b}_{\perp}>}=0 .
$$

A condição acima é equivalente a impor que o elemento de matriz da hamiltoniana, entre os estados do vácuo e de um fonon deformado ortogonalizados pelo método de Gram-Schmidt, projetados em momento angular L se anule: 


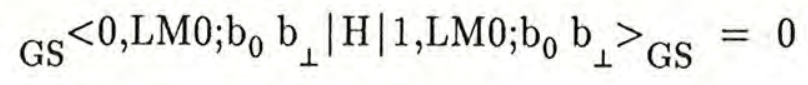

onde

$$
\begin{aligned}
& \left|0, \mathrm{LM} 0 ; \mathrm{b}_{0} \mathrm{~b}_{\perp}\right\rangle_{\mathrm{GS}}=\mid 0, \mathrm{LM} 0 ; \mathrm{b}_{0} \mathrm{~b}_{\perp}> \\
& \left|1, \mathrm{LM} 0 ; \mathrm{b}_{0} \mathrm{~b}_{\perp}>_{\mathrm{GS}}=\right| 1, \mathrm{LM} 0 ; \mathrm{b}_{0} \mathrm{~b}_{\perp}>-\mid 0, \mathrm{LM} 0 ; \mathrm{b}_{0} \mathrm{~b}_{\perp}> \\
& \cdot \frac{<0, \mathrm{LM} 0 ; \mathrm{b}_{0} \mathrm{~b}_{\perp} \mid 1, \mathrm{LM} 0 ; \mathrm{b}_{0} \mathrm{~b}_{\perp}>}{<0, \mathrm{LM} 0 ; \mathrm{b}_{0} \mathrm{~b}_{\perp} \mid 0, \mathrm{LM} 0 ; \mathrm{b}_{0} \mathrm{~b}_{\perp}>} .
\end{aligned}
$$

A demonstração da condição acima, segue da derivação explicita da expressão (8) e do uso da expressão

$$
\frac{\partial}{\partial \mathrm{b}_{\perp}}\left|0, \mathrm{LM} 0 ; \mathrm{b}_{0} \mathrm{~b}_{\perp}\right\rangle=\frac{\sqrt{2 \mathrm{k}_{\perp}}}{\mathrm{b}_{\perp}}\left|1, \mathrm{LM} 0 ; \mathrm{b}_{0} \mathrm{~b}_{\perp}\right\rangle
$$

que é obtida facilmente da expressão (3).

Dados os estados $\left|\mathrm{n}, \mathrm{LM} 0 ; \mathrm{b}_{0} \mathrm{~b}_{\perp}\right\rangle$ uma base ortogonal $\left|\mathrm{n}, \mathrm{LM} 0 ; \mathrm{b}_{0} \mathrm{~b}_{\perp}\right\rangle_{\mathrm{GS}}$, pode ser obtida através do método de Gram-Schmidt.

\subsubsection{Modelo $\mathrm{Sp}_{\perp}(1, \mathrm{R})$ Deformado Determinado pelo Estado de Peso Mínimo op-ob}

Até o presente momento, temos discutido de uma maneira geral como construir uma base deformada equivalente à base esférica, para o modelo $\mathrm{Sp}_{\perp}(1, \mathrm{R})$. A seguir analisaremos especificamente o cálculo no modelo $\mathrm{Sp}_{\perp}(1, \mathrm{R})$ em uma base deformada, equivalente ao cálculo $\mathrm{Sp}_{\perp}(1, \mathrm{R})$ que realizamos na seção 4.5.1., em uma base esférica. 
A partir dos estados de base esféricos do modelo $\mathrm{Sp}_{\perp}(1, \mathrm{R}),\left|\mathrm{n}(04), \mathrm{LM} 0 ; \mathrm{b}_{0}\right\rangle$, na notação da seção 4.3.1., obtemos os estados da base deformada equivalente, $\left.\ln (04), \mathrm{LM} 0 ; \mathrm{b}_{0} \mathrm{~b}_{\perp}\right\rangle$. Uma base ortogonal $\left|\mathrm{n}(04), \mathrm{LM} 0 ; \mathrm{b}_{0} \mathrm{~b}_{\perp}\right\rangle_{\mathrm{GS}}$ é obtida através do método de Gram-Schmidt.

O procedimento que utilizamos para obter os elementos de matriz dos operadores de interesse na base deformada, consiste em utilizar a expansão desta na base esférica através da expressão (5), truncando a base esférica em $n_{\operatorname{máx}}=34$ e então ortogonalizando pelo método de Gram-Schmidt os estados da base deformada. Verificamos que o truncamento que fizemos foi suficiente para garantir que os elementos de matriz dos operadores de interesse não se alteram significativamente para estados até 9-fonons. 0 nosso procedimento consiste em uma extensão para $n \neq 0$ do método proposto por Asherova et al $(20)$.

Na tabela 5.1 mostramos os valores de $b_{\perp}(L)$, que minimizam o valor esperado da hamiltoniana no estado do vácuo deformado, para $\mathrm{L}=0,2,4$. A função de onda obtida pela diagonalização da hamiltoniana em uma base com $\mathrm{N}$-fonons deformados é dada por:

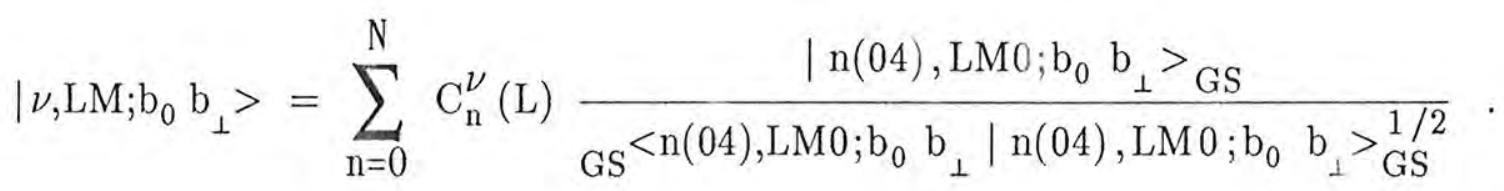

Na tabela 5.2 mostramos os valores de $\left|\mathrm{C}_{\mathrm{n}}^{\nu}(\mathrm{L})\right|^{2}$ que correspondem a probabilidade de encontrarmos nos dois primeiros estados de um dado $\mathrm{L}$, o estado do vácuo, de 1-fonon e 2-fonons projetados em momento angular $\mathrm{L}$ e ortogonalizados pelo método de Gram-Schmidt. Utilizamos na construção da tabela 2 uma base deformada com $\mathrm{N}=9$ fonons, o que foi suficiente para obtermos convergência segundo o mesmo critério 
que discutimos na seção 4.5 do capítulo anterior. Na tabela 5.3 mostramos a energia de excitação e as propriedades geométricas dos estados da banda fundamental calculados através da diagonalização da hamiltoniana na base deformada truncada em $\mathrm{N}=0-5$. $\mathrm{Na}$ mesma tabela apresentamos o cálculo exato, que obtivemos no caṕ́tulo anterior.

Nas figuras 5.1-5.3 mostramos a contribuição parcial dos estados |n(04),LM0; $\mathrm{b}_{0} \mathrm{~b}_{\perp}>_{\mathrm{GS}}$ ao momento de quadrupolo elétrico do primeiro estado $2^{+}$e ao $\mathrm{B}\left(\mathrm{E} 2,2^{+} \rightarrow 0^{+}\right)$entre estados da banda rotacional fundamental e da primeira banda excitada. Estes gráficos são análogos aos obtidos no capítulo anterior, figuras 4.3-4.5.

A tabela 5.2 nos mostra que os primeiros dois estados de um dado L são dominados pela projeção em momento L do estado de vácuo e de 1-fonon deformado, ortogonalizados pelo processo de Gram-Schmidt respectivamente. Notamos nesta tabela que a probabilidade de encontrarmos nas funções de onda do primeiro estado $0^{+}$e $2^{+}$o estado do vácuo projetado é maior do que $99 \%$. A dominância acima não é tão acentuada quando consideramos a probabilidade de encontrarmos o estado de 1-fonon nas funções de onda dos estados da primeira banda excitada. A contribuição da projeção do vácuo para o $\mathrm{B}\left(\mathrm{E} 2,2_{1}^{+} \rightarrow 0_{1}^{+}\right)$e $\mathrm{Q}_{\mathrm{el}}\left(2_{1}^{+}\right)$é maior do que $98 \%$.

Em todos os casos a contribuição vinda de estados com mais de cinco fonons é desprezível, como mostram as figuras 5.1-5.3. Isto é demonstrado de maneira mais evidente através da tabela 5.3, onde truncamos a base (10) em $N=0-5$. Para cada valor de $\mathrm{N}$, diagonalizamos a hamiltoniana e calculamos a energia de excitação e as propriedades geométricas dos estados da banda rotacional fundamental. Observamos que considerando apenas o estado do vácuo, obtemos resultados que diferem em menos de $2 \%$ dos resultados exatos, sendo os BE2 os observáveis mais sensíveis.

Concluindo, vemos que os estados da banda rotacional fundamental são bem descritos pela projeção em momento angular de um único estado, o vácuo do fonon transversal deformado. Estes fonons deformados são associados às ressonâncias gigantes 


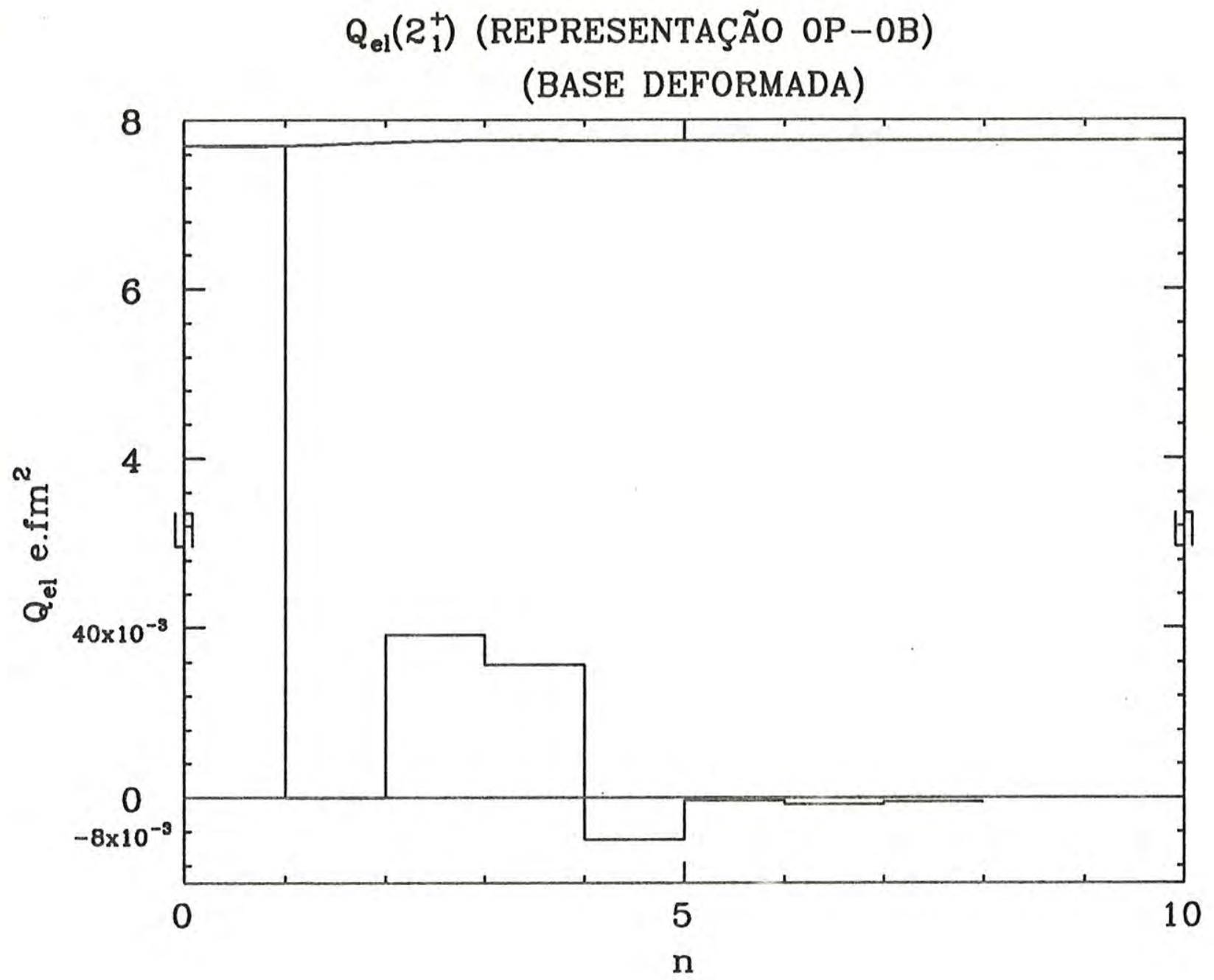

Fig. 5.1. Contribuição parcial ao $\mathrm{Q}_{\mathrm{el}}\left(2_{1}^{+}\right)$devido à inclusão sistemática, na expansão (5.10), dos estados de base do modelo $\mathrm{Sp}_{\perp}(1, \mathrm{R})$ deformado. O valor do $\mathrm{Q}_{\mathrm{el}}\left(2_{1}^{+}\right)$no ponto $\mathrm{n}$,é obtido considerando-se na expansão (10), a contribuição de todos os termos até $\mathrm{n}$, inclusive. Note a mudança na escala. 


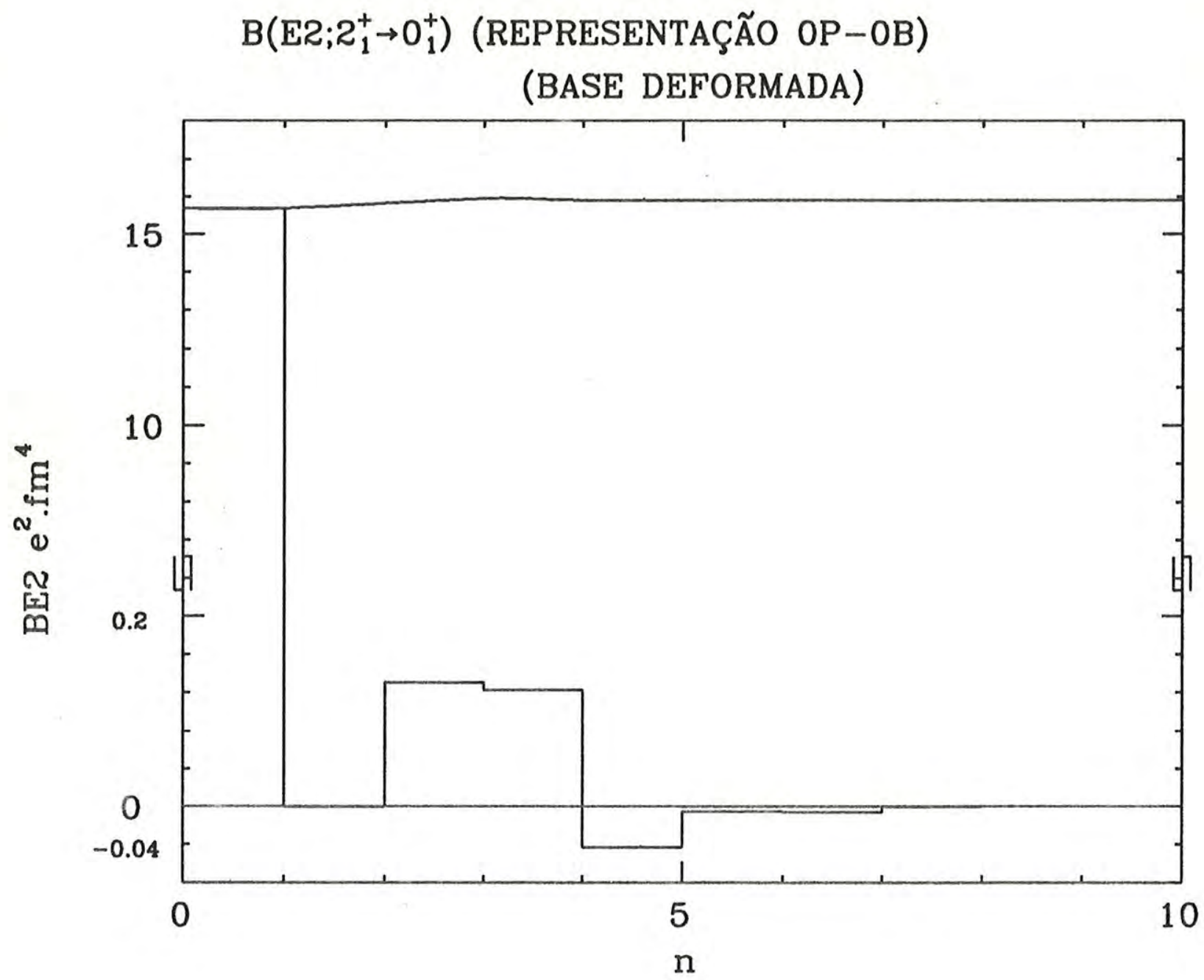

Fig. 5.2. Contribuição parcial ao $\mathrm{B}\left(\mathrm{E} 2,2_{1}^{+} \rightarrow 0_{1}^{+}\right)$, devido à inclusão sistemática dos estados de base do modelo $\mathrm{Sp}_{\perp}(1, \mathrm{R})$ deformado na expansão (5.10) da função de onda. O valor do B(E2) no ponto $n$,é obtido considerando-se na expansão (10), a contribuição de todos os termos até $n$, inclusive. Note a mudança na escala. 


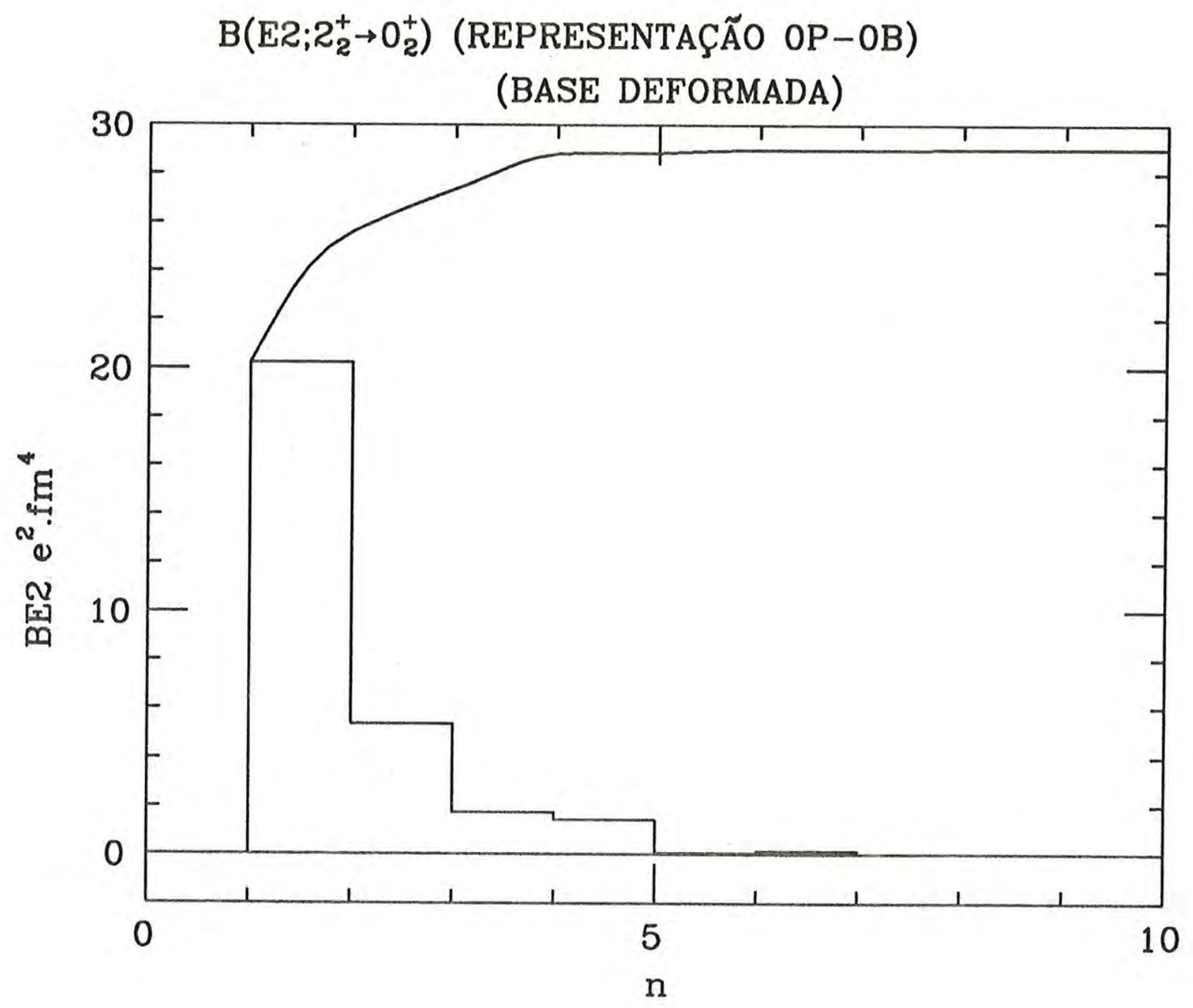

Fig. 5.3. Contribuição parcial ao $\mathrm{B}\left(\mathrm{E} 2,2_{2}^{+} \rightarrow 0_{2}^{+}\right)$, devido à inclusão sistemática dos estados de base do modelo $\mathrm{Sp}_{\perp}(1, \mathrm{R})$ deformado na expansão (5.10) da função de onda. O valor do B(E2) no ponto n é obtido considerando-se na expansão (10), a contribuição de todos os termos até $n$, inclusive. 
(representação $0 \mathrm{p}-0 \mathrm{~b})$

\begin{tabular}{lccc}
\hline & $\mathrm{L}=0$ & $\mathrm{~L}=2$ & $\mathrm{~L}=4$ \\
\hline $\mathrm{b}_{\perp}(\mathrm{fm})$ & 1.95 & 1.93 & 1.90 \\
\hline
\end{tabular}

Tab. 5.1. Valores de $b_{\perp}(L)$ que minimizam o valor esperado da hamiltoninana, no vácuo do fonon transversal deformado projetado em momento angular $\mathrm{L} \cdot\left(\mathrm{b}_{0}=1.36 \mathrm{fm}\right)$.

(representação $0 \mathrm{p}-0 \mathrm{~b}$ )

\begin{tabular}{ccccccc}
\hline & \multicolumn{2}{c}{$\left|\mathrm{C}_{\mathrm{n}}^{\nu}(0)\right|^{2}$} & & $\left|\mathrm{C}_{\mathrm{n}}^{\nu}(2)\right|^{2}$ & \multicolumn{2}{c}{$\left|\mathrm{C}_{\mathrm{n}}^{\nu}(4)\right|^{2}$} \\
\hline $\mathrm{n}^{\nu}$ & 1 & 2 & 1 & 2 & 1 & 2 \\
\hline 0 & 0.998 & - & 0.997 & - & 0.993 & 0.001 \\
1 & - & 0.937 & - & 0.927 & - & 0.904 \\
2 & 0.001 & 0.045 & 0.002 & 0.049 & 0.006 & 0.051 \\
\hline
\end{tabular}

Tab. 5.2. Probabilidade de encontrarmos o estado com $\mathrm{n}=0,1,2$ na expansão (5.10) dos primeiros dois estados $(\nu=1,2)$, com $\mathrm{L}=0,2,4$. (base deformada com $\mathrm{N}=9$ ). 
(representação $0 \mathrm{p}-0 \mathrm{~b})$

\begin{tabular}{|c|c|c|c|c|c|c|c|c|}
\hline \multicolumn{5}{|c|}{ EXATO } & \multicolumn{4}{|c|}{ VÁCUO } \\
\hline $\mathrm{L}$ & \multicolumn{2}{|c|}{ E.EXC. $<\mathrm{r}^{2}>^{1 / 2}$} & $\mathrm{BE} 2$ & $\mathrm{Q}_{\mathrm{el}}$ & \multicolumn{2}{|c|}{ E.EXC. $<\mathrm{r}^{2}>^{1 / 2}$} & $\mathrm{BE} 2$ & $\mathrm{Q}_{\mathrm{el}}$ \\
\hline 0 & $\longrightarrow$ & 2.60 & - & $\longrightarrow$ & \multicolumn{2}{|r|}{2.60} & - & - \\
\hline 2 & 3.07 & 2.59 & 15.89 & 7.75 & 3.09 & 2.59 & 15.70 & 7.70 \\
\hline 4 & 11.59 & 2.58 & 23.01 & 8.70 & 11.71 & 2.57 & 22.55 & 8.54 \\
\hline \multicolumn{4}{|c|}{2 -fonons } & & \multicolumn{4}{|c|}{ 3-fonons } \\
\hline $\mathrm{L}$ & \multicolumn{2}{|c|}{ E.EXC. $<\mathrm{r}^{2}>^{1 / 2}$} & $\mathrm{BE} 2$ & $\mathrm{Q}_{\mathrm{el}}$ & \multicolumn{2}{|c|}{ E.EXC. $<\mathrm{r}^{2}>^{1 / 2}$} & BE2 & $\mathrm{Q}_{\mathrm{el}}$ \\
\hline 0 & $\longrightarrow$ & 2.60 & - & - & - & 2.60 & - & - \\
\hline 2 & 3.07 & 2.59 & 15.83 & 7.74 & 3.07 & 2.59 & 15.95 & 7.77 \\
\hline \multirow[t]{2}{*}{4} & 11.55 & 2.58 & 22.81 & 8.65 & 11.57 & 2.58 & 23.04 & 8.69 \\
\hline & \multicolumn{3}{|c|}{ 4-fonons } & & \multicolumn{4}{|c|}{ 5-fonons } \\
\hline $\mathrm{L}$ & \multicolumn{2}{|c|}{ E.EXC. $<r^{2}>^{1 / 2}$} & BE2 & $\mathrm{Q}_{\mathrm{el}}$ & \multicolumn{2}{|c|}{ E.EXC. $<\mathrm{r}^{2}>^{1 / 2}$} & BE2 & $\mathrm{Q}_{\mathrm{el}}$ \\
\hline 0 & - & 2.60 & - & - & - & 2.60 & - & - \\
\hline 2 & 3.07 & 2.59 & 15.91 & 7.76 & 3.07 & 2.59 & 15.90 & 7.75 \\
\hline 4 & 11.58 & 2.58 & 23.01 & 8.70 & 11.59 & 2.58 & 23.01 & 8.70 \\
\hline
\end{tabular}

Tab. 5.3. Níveis de energia de excitação e propriedades geométricas dos estados da banda rotacional fundamental calculados em uma base deformada truncada em $\mathrm{N}=0-5$. Energias em $\mathrm{MeV},\left\langle\mathrm{r}^{2}>^{1 / 2}\right.$ em $\mathrm{fm}, \mathrm{BE} 2(\mathrm{~L} \rightarrow \mathrm{L}-2)$ em $\mathrm{e}^{2} \cdot \mathrm{fm}^{4}$ e $\mathrm{Q}_{\mathrm{el}}$ em $\mathrm{e} \cdot \mathrm{fm}^{2}$. 
monopolares e quadrupolares.

Portanto o modelo $\mathrm{Sp}_{\perp}(1, \mathrm{R})$ pode ser visto como uma generalização do modelo projeção antes da variação de um oscilador harmônico deformado $(10),(11)$.

\subsection{Modelo $\mathrm{Sp}_{\| 1}(1, \mathrm{R})$ Deformado}

Nesta seção vamos considerar o modelo $\operatorname{Sp}_{\| \prime}(1, R)$ em uma base deformada. A distinção entre os casos associados aos estados de peso mínimo $2 \mathrm{p}-2 \mathrm{~b}$ e $4 \mathrm{p}-4 \mathrm{~b}$ faremos nas sub-seções seguintes. Analogamente à seção 5.2, a partir da base de estados (4.7), construída em termos de funções de onda do oscilador harmônico esférico e da. transformação de escala na direção longitudinal, gerada pelo operador:

$$
\mathrm{S}_{11}=\mathrm{i}\left[\mathrm{A}_{\|}^{+}\left(\mathrm{b}_{0}\right)-\mathrm{A}_{11}\left(\mathrm{~b}_{0}\right)\right]
$$

vamos construir a base deformada.

A partir das relações (1) e (2) segue que a transformação de escala gerada por $S_{\| \text {, }}$, aplicada ao estado (4.7)

$$
\left|n ; b_{11} b_{0}>=e^{-i \ln \left(b_{11} / b_{0}\right) S_{11}}\right| n ; b_{0}>
$$


e aos geradores da álgebra $\mathrm{sp}_{11}(1, \mathrm{R}),(4.5)$ :

$$
\left\{\begin{array}{l}
A_{11}^{+}\left(b_{11}\right) \\
A_{11}\left(b_{11}\right) \\
A_{011}\left(b_{11}\right)
\end{array}\right\}=e^{-i \ln \left(b_{11} / b_{0}\right) S_{11}}\left\{\begin{array}{l}
A_{11}^{+}\left(b_{0}\right) \\
A_{11}\left(b_{0}\right) \\
A_{011}\left(b_{0}\right)
\end{array}\right\} e^{i \ln \left(b_{11} / b_{0}\right) S_{11}}
$$

resulta em:

$$
\begin{aligned}
& \left|n ; b_{11} b_{0}>=\left[A_{11}^{+}\left(b_{11}\right)\right]^{n}\right| 0 ; b_{11} b_{0}>\sqrt{\frac{\Gamma\left(2 k_{11}\right)}{\Gamma(n+1) \Gamma\left(n+2 k_{11}\right)}} \\
& A_{11}^{+}\left(b_{11}\right)=\frac{1}{2} \sum_{k=1}^{A-1} a_{1 ; k}^{+}\left(b_{11}\right) a_{1 ; k}^{+}\left(b_{11}\right) \\
& A_{11}\left(b_{11}\right)=\left[A_{11}^{+}\left(b_{11}\right)\right]^{+} \\
& A_{011}\left(b_{11}\right)=\frac{1}{2} C_{11}\left(b_{11}\right)=\frac{1}{4} \sum_{k=1}^{A-1}\left[a_{1 ; k}^{+}\left(b_{11}\right) a_{1 ; k}\left(b_{11}\right)+a_{1 ; k}\left(b_{11}\right) a_{1 ; k}^{+}\left(b_{11}\right)\right] .
\end{aligned}
$$

Os operadores $A_{11}^{+}\left(b_{11}\right), A_{11}\left(b_{11}\right)$ e $A_{011}\left(b_{11}\right)$ formam uma base equivalente da álgebra $\mathrm{sp}_{\| 1}(1, \mathrm{R})$, dada em $(4.5)$ e os estados $\left|\mathrm{n} ; \mathrm{b}_{\|} \mathrm{b}_{0}\right\rangle$ uma base de estados equivalente da representação irredutível $k_{\|}$de $S_{n}(1, R)$. O efeito da transformação de escala em $\left|\mathrm{n} ; \mathrm{b}_{0}\right\rangle$ é substituir as funções de onda do oscilador harmônico esférico onde $b_{x}=b_{y}=b_{z}=b_{0}$ pelas funções de onda do oscilador harmônico deformado onde $b_{x}=b_{y}=b_{0}$ e $b_{z}=b_{11}$. 
Analogamente ao caso esférico os estados $\left|\mathrm{n} ; \mathrm{b}_{\|} \mathrm{b}_{0}\right\rangle$ podem ser interpretados como estados de $\mathrm{n}$-fonons deformados.

Os coeficientes da expansão de $\left|n ; b_{\|} b_{0}\right\rangle$ nos estados da base esférica $\left|n ; b_{0}\right\rangle$ :

$$
\left|n ; b_{11} b_{0}\right\rangle=\sum_{n^{\prime}} \mid n^{\prime} ; b_{0}>U_{n^{\prime} n}
$$

podem ser calculados explicitamente

$$
\mathrm{U}_{\mathrm{n}^{\prime} \mathrm{n}}=\left\langle\mathrm{n}^{\prime} ; \mathrm{b}_{0}\left|\mathrm{e}^{-\mathrm{i} \ln \left(\mathrm{b}_{11} / \mathrm{b}_{0}\right) \mathrm{S}_{\text {" }}}\right| \mathrm{n} ; \mathrm{b}_{0}\right\rangle .
$$

A expressão para $U_{\text {n'n }}$ é obtida de (6) trocando-se:

$$
\begin{aligned}
& \mathrm{b}_{\perp} \rightarrow \mathrm{b}_{11} \\
& \mathrm{k}_{\perp} \rightarrow \mathrm{k}_{11} \\
& \delta_{\perp} \rightarrow \delta_{11}=\frac{\mathrm{b}_{11}^{2}-\mathrm{b}_{0}^{2}}{\mathrm{~b}_{11}^{2}+\mathrm{b}_{0}^{2}} .
\end{aligned}
$$

Através da projeção em momento angular dos estados (13) obtemos a relação entre as duas bases equivalentes do modelo $\mathrm{Sp}_{11}(1, \mathrm{R})$ :

$$
\left|n, K L M ; b_{\|} b_{0}>=\sum_{n^{\prime}}\right| n^{\prime}, K L M ; b_{0}>U_{n^{\prime} n} .
$$


Os estados acima não são ortogonais nos rótulos $K$ e $n$. Vamos a seguir considerar separadamente os casos onde temos bases axialmente simétricas e triaxiais.

\section{a) Caso Axialmente Simétrico}

Neste caso o estado de peso mínimo $|0\rangle$ é prolato, axialmente simétrico e tem rótulos de Elliott $(\lambda 0)$. Portanto, só temos estados de base com $K=0$ e a base de estados (14) é não ortogonal apenas no rótulo n . A base é linearmente dependente (L.D.) se $L>\lambda$, onde o número de estados L.D. é igual a $n_{L}=\frac{L-\lambda}{2}$.

A base (14) depende do parâmetro $b_{\|}$, que é determinado de modo análogo à seção 5.2, ou seja, pelo método da projeção antes da variação. Como este caso é inteiramente análogo ao discutido na seção 5.2 não vamos reconsiderá-lo.

\section{b) Caso Triaxial}

Neste caso o estado de peso mínimo de $\operatorname{Sp}_{\|}(1, R)$ não é axialmente simétrico e tem rótulos de Elliott $(\lambda \mu) \operatorname{com} \lambda>\mu$, como discutimos no capítulo 4. Portanto, a base de estados (14), agora também vai envolver estados com $K \neq 0$. Os estados (14) não são ortogonais nos rótulos $\mathrm{K}$ e $\mathrm{n}$ e são linearmente dependentes para $\mathrm{L}>\lambda+\mathrm{K}$, onde $\mathrm{K}$ é dado pela regra de Elliott, que mostramos no capítulo 4 . O número de estados linearmente depedentes é dado por:

$$
\mathrm{n}_{\mathrm{L}}^{\mathrm{K}}=\frac{[\mathrm{L}]-(\lambda+\mathrm{K})}{2}
$$

onde $[\mathrm{L}]=\mathrm{L}+1$ se $\mathrm{L}-\lambda-\mathrm{K}$ é ímpar e $[\mathrm{L}]=\mathrm{L}$ se $\mathrm{L}-\lambda-\mathrm{K}$ é par. Para $\mathrm{K}=0$ a expressão acima se reduz a $\mathrm{n}_{\mathrm{L}}^{0}=\frac{\mathrm{L}-\lambda}{2}$.

A base de estados deformados (14) depende do parâmetro $b_{\|}$e como para cada valor de " $n$ " temos vários estados devido à projeção em $K$, precisamos generalizar o 
procedimento utilizado na última seção, referente ao caso axialmente simétrico. Agora o comprimento do oscilador $b_{11}$ vai depender de $K$, ou seja, teremos $b_{11}(K)$.

$\mathrm{Na}$ determinação de $\mathrm{b}_{11}(\mathrm{~K})$, vamos procurar utilizar um método variacional análogo ao caso axialmente simétrico. Assim sendo, vamos construir a partir do estado do vácuo deformado, o estado mais geral possível, consistente com a invariância rotacional, isto é:

$$
\left|\tau \mathrm{LM}>=\sum_{\mathrm{K}} \mathrm{C}_{\mathrm{K}}^{\tau}\right| 0, \mathrm{LMK} ; \mathrm{b}_{11}(\mathrm{~K}) \mathrm{b}_{0}>
$$

onde os coeficientes $\mathrm{C}_{\mathrm{K}}^{\tau}$ são obtidos através da diagonalização da hamiltoniana na base não ortogonal $\mid 0, L M K ; b_{11}(K) b_{0}>$. Notemos que $C_{K}^{\tau}$ também é função de $b_{11}(K)$. Portanto fixamos os parâmetros $b_{11}(K)$ de modo que a energia para cada valor de $L$ do estado mais baixo $\mid \tau$ LM> seja um mínimo com função de $\mathrm{b}_{11}(\mathrm{~K})$ :

$$
\frac{\partial}{\partial \mathrm{b}_{\mathrm{II}}(\mathrm{K})} \frac{<\tau \mathrm{LM}|\mathrm{H}| \tau \mathrm{LM}>}{<\tau \mathrm{LM} \mid \tau \mathrm{LM}>}=0
$$

Podemos mostrar que a condição acima equivale a afirmar que o elemento de matriz da hamiltoniana entre o estado $|\tau \mathrm{LM}\rangle$ e o estado $\left|1, \mathrm{LMK} ; \mathrm{b}_{11}(\mathrm{~K})\right\rangle$ ortogonalizado pelo processo de Gram-Schmidt em relação ao estado $\mid \tau \mathrm{LM}>$ é nulo para todos os valores possíveis de $\mathrm{K}$, isto é:

$$
<\tau \mathrm{LM}|\mathrm{H}| 1, \mathrm{LMK} ; \mathrm{b}_{11}(\mathrm{~K}) \mathrm{b}_{0}>_{\mathrm{GS}}=0
$$

onde

$$
\left|1, \mathrm{LMK} ; \mathrm{b}_{\|}(\mathrm{K}) \mathrm{b}_{0}>_{\mathrm{GS}}=\right| 1, \mathrm{LMK} ; \mathrm{b}_{\| 1}(\mathrm{~K}) \mathrm{b}_{0}>-\frac{|\tau \mathrm{LM}><\tau \mathrm{LM}| 1, \mathrm{LMK} ; \mathrm{b}_{\| 1}(\mathrm{~K}) \mathrm{b}_{0}>}{<\tau \mathrm{LM} \mid \tau \mathrm{LM}>} .
$$


A base de estados do modelo $|\mathrm{n}, \mathrm{LM} \overline{\mathrm{k}}\rangle_{\mathrm{GS}}$ é obtida ortonormalizando pelo procedimento de Gram-Schmidt os estados não ortogonais $\left|\mathrm{n}, \mathrm{LMK} ; \mathrm{b}_{11}(\mathrm{~K}) \mathrm{b}_{0}\right\rangle$, e podemos mostrar que a condição de mínimo dada acima, implica que os elementos de matriz entre o estado $\mid \tau \mathrm{LM}>$ de menor energia e os estados de base do modelo com $\mathrm{n}=1$ sejam nulos.

\subsubsection{Modelo $\mathrm{Sp}_{\|}(1, \mathrm{R})$ Deformado Determinado pelo Estado de Peso Mínimo 2p-2b}

A seguir analisaremos o cálculo no modelo $\mathrm{Sp}_{11}(1, \mathrm{R})$ deformado, associado ao estado de peso mínimo $2 \mathrm{p}-2 \mathrm{~b}$. Este cálculo é equivalente ao realizado na seção 4.5 .2 onde utilizamos uma base de estados esférica. No capítulo 4 mostramos que devido a regra de Elliott, a projeção em momento angular vai envolver apenas estados com $\mathrm{K}=0,2$ e no mesmo capítulo mostramos os possíveis valores do momento angular orbital $\mathrm{L}$.

A partir dos estados de base esféricos $\left|\mathrm{n}(62), \mathrm{LMK} ; \mathrm{b}_{0}\right\rangle$, que discutimos na seção 4.3.2, geramos os estado deformados $\mid \mathrm{n}(62), \mathrm{LMK} ; \mathrm{b}_{11}(\mathrm{~K}) \mathrm{b}_{0}>, \mathrm{K}=0,2$. Utilizando 0 pıocesso de ortogonalização de Gram-Schmidt geramos a base de estados deformada ortogonal $\mid \mathrm{n}(62), \mathrm{LM} \overline{\mathrm{k}}>_{\text {GS }}$.

Em completa analogia com a seção 5.2 , os elementos de matriz dos operadores de interesse na base deformada foram obtidos através da expansão na base esférica truncada e por fim ortogonalizamos pelo processo de Gram-Schmidt.

A base esférica utilizada foi truncada em $n_{\text {máx }}=34$ o que assegurou que os elementos de matriz dos operadores de interesse não se alteraram significativamente para bases com até 9 fonons deformados.

$\mathrm{Na}$ tabela 5.4 mostramos os valores de $\mathrm{b}_{11}(\mathrm{~K})$, para $\mathrm{L}=0,2,4$, que minimizam o valor esperado da hamiltoniana no estado obtido pela projeçào em momento angular L do 
estado do vácuo deformado como discutimos na última seção. Notemos que como $\mathrm{L}=0$, só pode ser projetado a partir de estados com $\mathrm{K}=0$, o procedimento neste caso é o mesmo que foi utilizado no caso axialmente simétrico.

A função de onda obtida através da diagonalização da hamiltoniana em uma base com $\mathrm{N}$-fonons deformados é dada por:

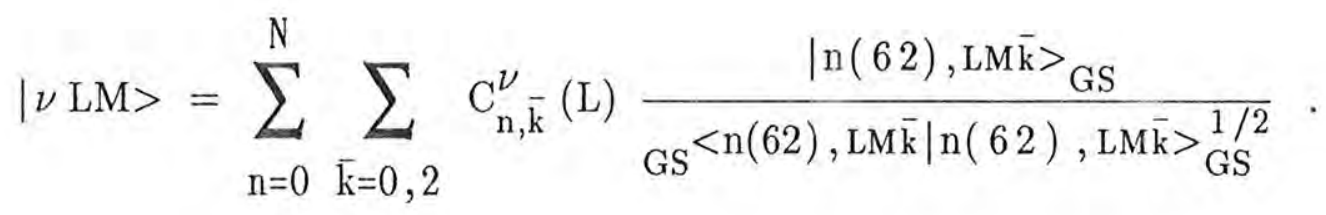

Na tabela 5.5 mostramos os valores de $\left|\mathrm{C}_{\mathrm{n}, \overline{\mathrm{k}}}^{\nu}(\mathrm{L})\right|^{2}$, que correspondem a probabilidade de encontrarmos nos dois estados de menor energia de um dado L, o estado do vácuo, 1-fonon, 2-fonons projetados em $\mathrm{L}, \mathrm{K},(\mathrm{K}=0,2)$ e ortogonalizados pelo processo de Gram-Schmidt.

$\mathrm{Na}$ tabela 5.6 mostramos a energia de excitação e as propriedades geométricas dos estados de energia mais baixa para $\mathrm{L}=0,2,4$, obtidas pela diagonalização da hamiltoniana na base deformada truncada em $\mathrm{N}=0-5$, onde sempre consideramos para cada $\mathrm{n}$ os dois possíveis valores de $\mathrm{K}$, quando $\mathrm{L} \neq 0$. Na mesma tabela apresentamos o cálculo exato que obtivemos no capítulo anterior.

Nas figuras 5.4-5.7 mostramos a contribuição parcial dos estados $|\mathrm{n}(62), \mathrm{LM} \overline{\mathrm{k}}\rangle_{\mathrm{GS}}$, $\overline{\mathrm{k}}=0,2$ ao momento de quadrupolo elétrico do primeiro estado com $\mathrm{L}=2$ ao $\mathrm{B}\left(\mathrm{E} 2,2_{\nu}^{+} \rightarrow 0_{1}^{+}\right), \nu=1,2$ e ao $\mathrm{B}\left(\mathrm{E} 2,2_{3}^{+} \rightarrow 0_{2}^{+}\right)$. Para cada $\mathrm{n}$, sempre que $\mathrm{L} \neq 0$, consideramos as duas possibilidades para $K$ na base de estados. Estes gráficos são análogos a.os obtidos no capítulo anterior, figuras 4.8-4.11.

A tabela 5.5 mostra que os primeiros dois estados de um dado $\mathrm{L}$, são dominados 
pela projeção em momento angular do estado do vácuo deformado. Nos casos onde temos $\mathrm{L} \neq 0$, devemos adicionar as probabilidades devido a $\overline{\mathrm{k}}=0$ e $\overline{\mathrm{k}}=2$. Notemos que a probabilidade de encontrarmos a projeção do vácuo deformado na função de onda do primeiro estado com $\mathrm{L}=0,2,4$ é superior a $99 \%$.

A figura 5.7 nos mostra que mesmo para os estados excitados, poucos fonons deformados são suficientes para descrevermos os operadores envolvidos, em geral menos de cinco. A tabela 5.6 nos mostra que a contribuição vinda de estados com mais de 5 -fonons é pequena e que o $\mathrm{B}(\mathrm{E} 2)$ e o $\mathrm{Q}_{\mathrm{el}}$ são os operadores mais sensíveis. Se considerarmos uma base deformada consistindo apenas na projeção do vácuo deformado, com excessão do momento de quadrupolo elétrico, obtemos resultados que diferem em menos de $6 \%$ dos resultados exatos. O caso que apresenta a maior diferença em relação ao cálculo exato é o $\mathrm{Q}_{\mathrm{el}}\left(2_{1}^{+}\right)$, onde a projeção do vácuo não atinge $50 \%$ do valor exato. Vamos analisar em detalhe este caso a fim de entendermos este comportamento.

Quando consideramos na expansão (5.15) apenas $\mathrm{N}=0$, isto é, projetamos o estado do vácuo deformado, o momento de quadrupolo elétrico $\mathrm{Q}_{\mathrm{el}}(\mathrm{L})$ é dado por quatro termos, onde os dois termos diagonais que são multiplicados pelo fator $\left|\mathrm{C}_{0, \overline{\mathrm{k}}}^{1}(\mathrm{~L})\right|^{2}, \overline{\mathrm{k}}=0,2 \mathrm{em}$ geral são dominantes. 
No caso específico do $\mathrm{Q}_{\mathrm{el}}\left(2_{1}^{+}\right)$temos:

$$
\begin{aligned}
& <\tau=1 \mathrm{~L}=2 \mathrm{M}=2\left|\mathrm{Q}_{20}^{\mathrm{el}}\right| \tau=1 \mathrm{~L}=2 \mathrm{M}=2>= \\
& =\left|\mathrm{C}_{00}^{1}(2)\right|_{\mathrm{GS}}^{2}<0(62), 22 \overline{\mathrm{k}}=0\left|\mathrm{Q}_{20}^{\mathrm{el}}\right| 0(62), 22 \overline{\mathrm{k}}=0>_{\mathrm{GS}}+ \\
& +2 \mathrm{C}_{00}^{1}(2) \mathrm{C}_{02}^{1}(2)_{\mathrm{GS}}<0(62), 22 \overline{\mathrm{k}}=0\left|\mathrm{Q}_{20}^{\mathrm{el}}\right| 0(62), 22 \overline{\mathrm{k}}=2>_{\mathrm{GS}}+ \\
& +\left|\mathrm{C}_{02}^{1}(2)\right|_{\mathrm{GS}}^{2}<0(62), 22 \overline{\mathrm{k}}=2\left|\mathrm{Q}_{20}^{\mathrm{el}}\right| 0(62), 22 \overline{\mathrm{k}}=2>_{\mathrm{GS}}
\end{aligned}
$$

onde os coeficientes $\mathrm{C}_{0, \overline{\mathrm{k}}}^{1}(2), \overline{\mathrm{k}}=0,2$ são dado por:

\begin{tabular}{lcc}
\hline & $\overline{\mathrm{k}}=0$ & $\overline{\mathrm{k}}=2$ \\
\hline $\mathrm{C}_{0, \mathrm{k}}^{1}(2)$ & 0.77 & 0.64 \\
\hline
\end{tabular}

e os elementos de matriz de $Q_{20}^{\text {el }}$ são dados por: 


\begin{tabular}{ccc}
\hline$\overline{\mathrm{k}}_{1}$ & $\overline{\mathrm{k}}_{2}$ & $\mathrm{GS}^{<0(62), 22} \overline{\mathrm{k}}_{1}\left|\mathrm{Q}_{20}^{\mathrm{el}}\right| 0(62), 22 \overline{\mathrm{k}}_{2}>_{\mathrm{GS}}$ \\
\hline 0 & 0 & -17.5 \\
0 & 2 & 2.1 \\
2 & 0 & 2.1 \\
2 & 2 & 17.8 \\
\hline
\end{tabular}

Notemos que os elementos de matriz diagonais de $\mathrm{Q}_{\mathrm{el}}\left(2_{1}^{+}\right)$tem sinais opostos e por isto há um cancelamento dos termos dominantes. Assim sendo, no caso do $\mathrm{Q}_{\mathrm{el}}\left(2_{1}^{+}\right)$, ocorre que a contribuição de termos com $N \neq 0$ na expansão (15) passam a ser importantes.

É importante salientar que esta é uma situação especifica do caso $\mathrm{L}=2$, como vemos na tabela 5.6. Portanto concluímos que mesmo para o caso $2 \mathrm{p}-2 \mathrm{~b}$ a projeção do vácuo deformado ainda é uma boa aproximação para o modelo $\operatorname{Sp}_{\| 1}(1, \mathrm{R})$, como vemos na tabela 5.6. A partir da discussão acima, é claro que precisamos ter cuidado com operadores que possam ter grandes cancelamentos como o momento de quadrupolo elétrico. Na tabela 5.6 vemos que uma base deformada com 5 -fonons, difere em menos de $3 \%$ dos resultados exatos, e os $Q_{\mathrm{el}}$ e BE2 são os observáveis mais sensíveis. 


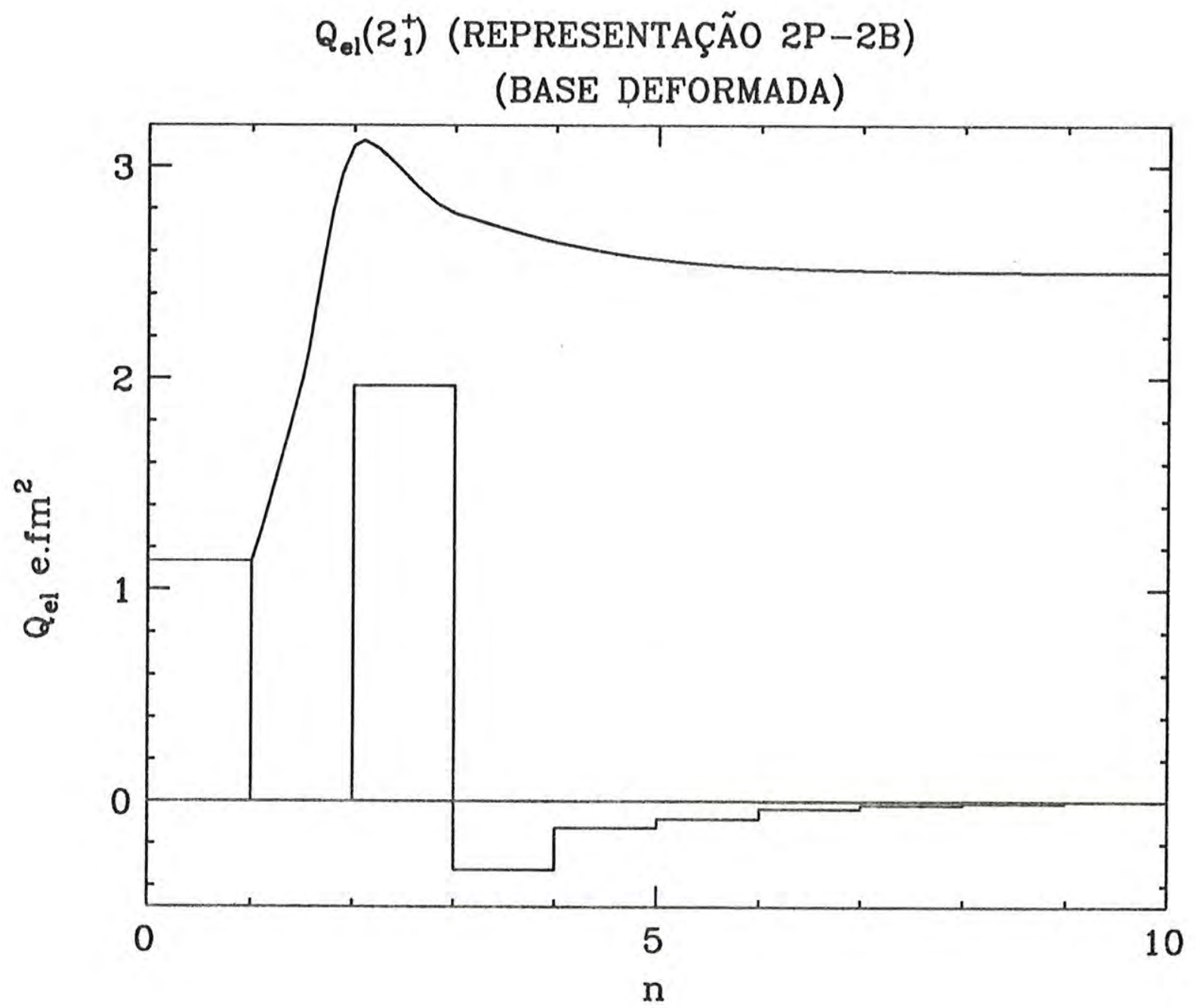

Fig. 5.4. Contribuição parcial ao $\mathrm{Q}_{\mathrm{el}}\left(2_{1}^{+}\right)$, devido à inclusão sistemática na expansão (5.15) dos estados de base do modelo $\operatorname{Sp}_{11}(1, R)$ deformado, onde sempre consideramos as duas possibilidades do rótulo $\overline{\mathrm{k}}$. O valor do $\mathrm{Q}_{\mathrm{el}}\left(2_{1}^{+}\right)$no ponto $n$,é obtido considerando-se na expansão (15), a contribuição de todos os termos até $n$, inclusive. 


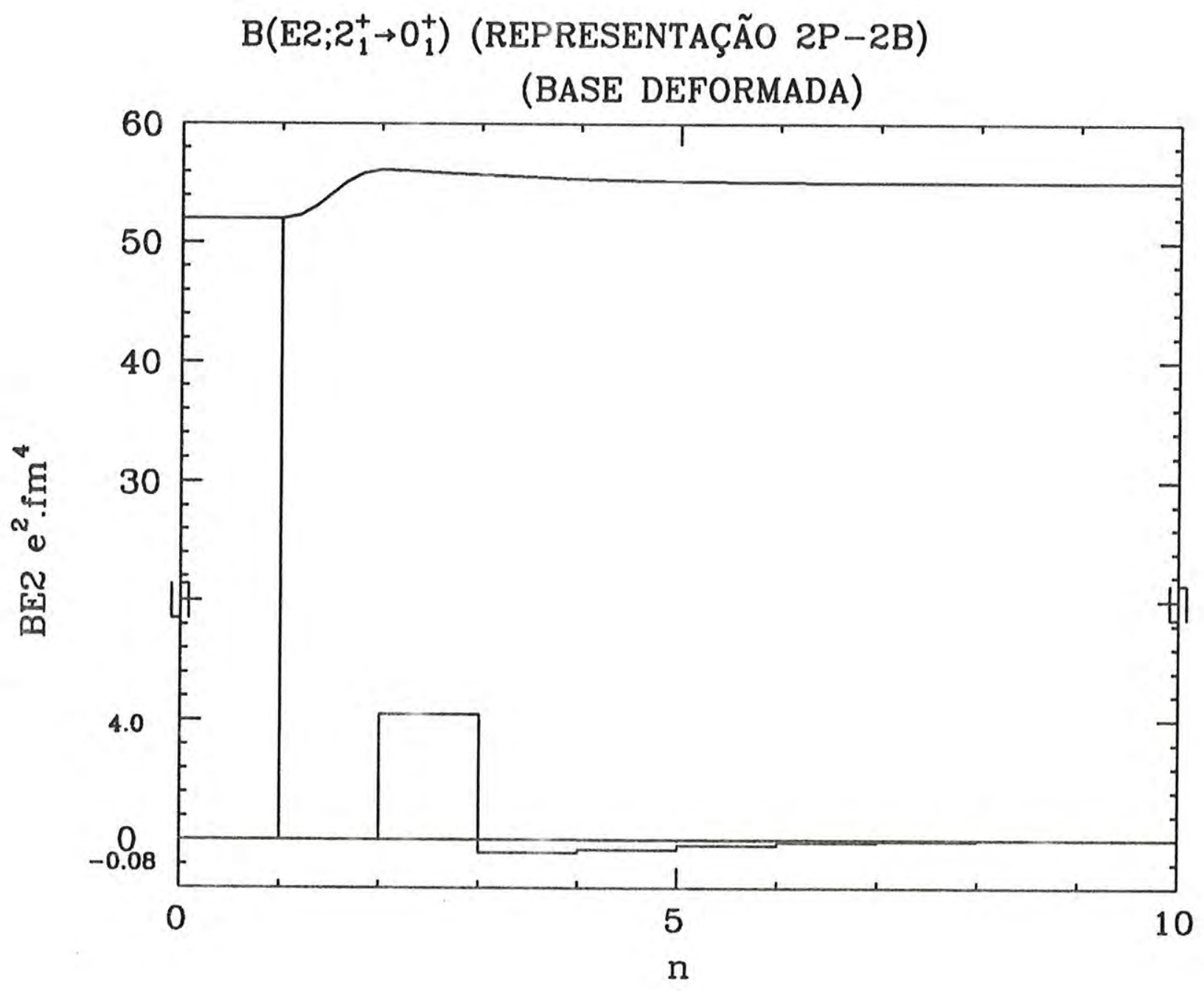

Fig. 5.5. Contribuição parcial ao $\mathrm{B}\left(\mathrm{E} 2,2_{1}^{+} \rightarrow 0_{1}^{+}\right)$, devido à inclusão sistemática na expansão (5.15) dos estados de base do modelo $\operatorname{Sp}_{11}(1, R)$ deformado, onde sempre consideramos as duas possibilidades do rótulo $\overline{\mathrm{k}}$. O valor do $\mathrm{B}(\mathrm{E} 2)$ no ponto $\mathrm{n}$,é obtido considerando-se na expansão (15), a contribuição de todos os termos até $n$, inclusive. (Note a mudança de escala) 


\section{$\mathrm{B}\left(\mathrm{E} 2 ; 2_{2}^{+} \rightarrow 0_{1}^{+}\right)$(REPRESENTAÇÃO $\left.2 \mathrm{P}-2 \mathrm{~B}\right)$ (BASE DEFORMADA)}

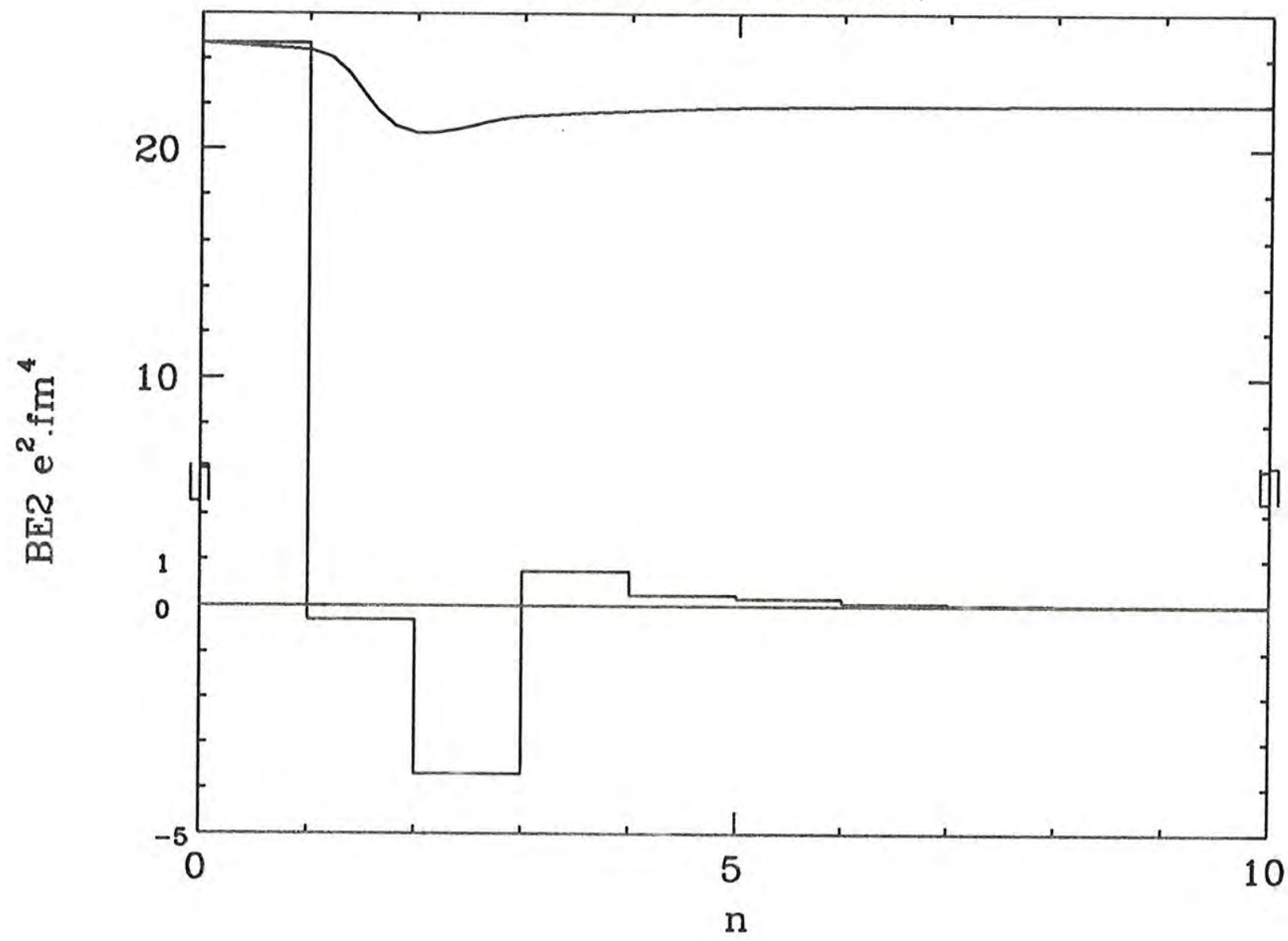

Fig. 5.6. Contribuição parcial ao $\mathrm{B}\left(\mathrm{E} 2,2_{2}^{+} \rightarrow 0_{1}^{+}\right)$, devido à inclusão sistemática na expansão (5.15) dos estados de base do modelo $\mathrm{Sp}_{\| 1}(1, \mathrm{R})$ deformado, onde sempre consideramos as duas possibilidades do rótulo $\overline{\mathrm{k}}$. O valor do $\mathrm{B}(\mathrm{E} 2)$ no ponto $n$,é obtido considerando--se na expansão (15), a contribuição de todos os termos até $n$, inclusive. (Note a mudança de escala) 


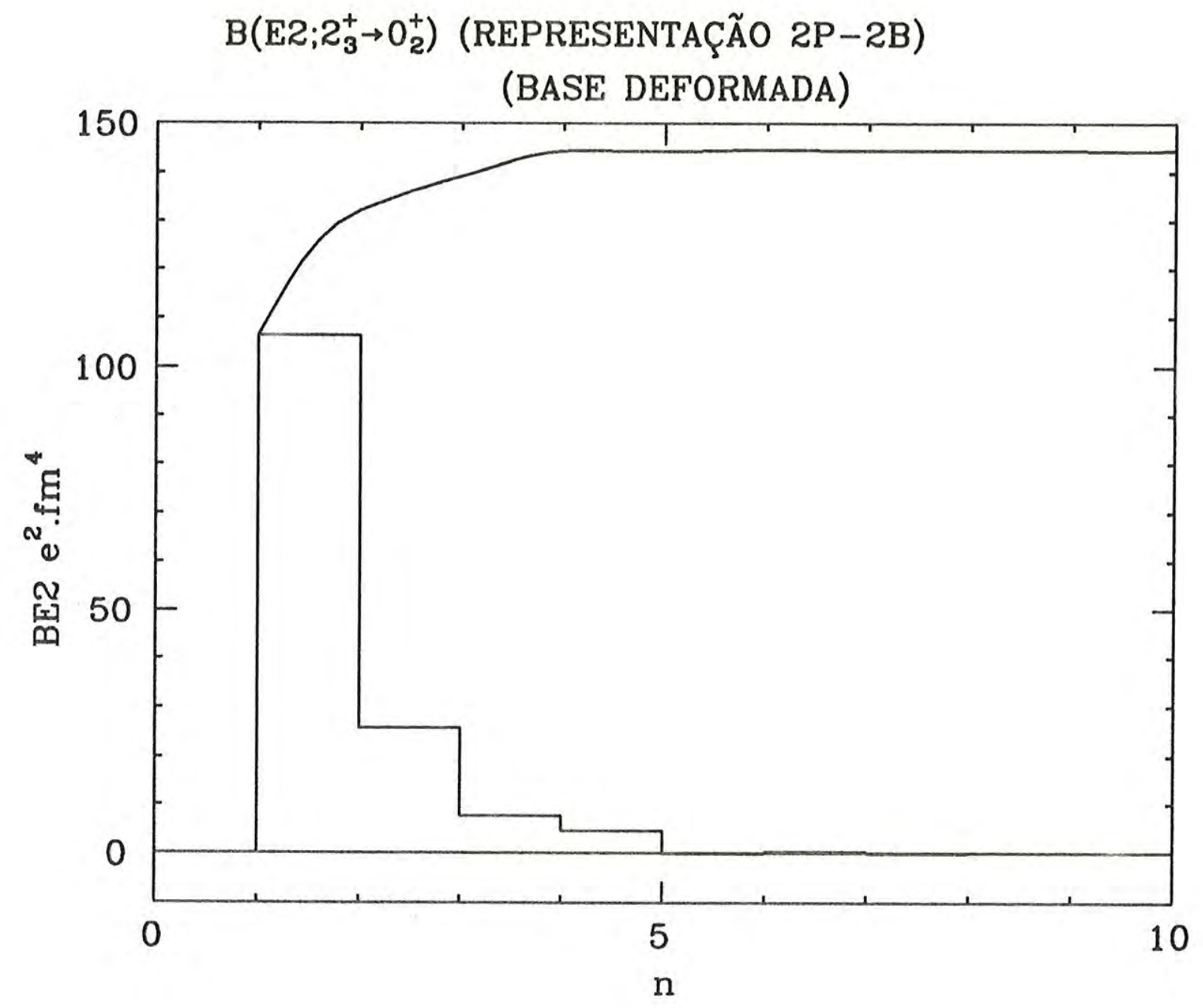

Fig. 5.7. Contribuição parcial ao $\mathrm{B}\left(\mathrm{E} 2,2_{3}^{+} \rightarrow 0_{2}^{+}\right)$, devido à inclusão sistemática na expansão (5.15) dos estados de base do modelo $\mathrm{Sp}_{11}(1, \mathrm{R})$ deformado, onde sempre consideramos as duas possibilidades do rótulo $\overline{\mathrm{k}}$. O valor do $\mathrm{B}(\mathrm{E} 2)$ no ponto $\mathrm{n}$,é obtido considerando-se na expansão (15), a contribuição de todos os termos até $\mathrm{n}$, inclusive. 
(representação $2 \mathrm{p}-2 \mathrm{~b}$ )

\begin{tabular}{cccccc}
\hline & $\mathrm{L}=0$ & $\mathrm{~L}=2$ & $\mathrm{~L}=4$ \\
\hline $\mathrm{K}=0$ & $\mathrm{~K}=2$ & $\mathrm{~K}=0$ & $\mathrm{~K}=2$ & $\mathrm{~K}=0$ & $\mathrm{~K}=2$ \\
\hline $\mathrm{b}$ & & & & & \\
\hline
\end{tabular}

Tab. 5.4. Valores de $b_{11}(K)$ que minimizam o valor esperado da hamiltoniana dos estados obtidos pela projeção em momento angular L do estado do vácuo como discutimos na seção $5.3\left(\mathrm{~b}_{0}=1.58 \mathrm{fm}\right)$. 


\begin{tabular}{|c|c|c|c|c|c|c|}
\hline & \multicolumn{2}{|c|}{$\left|\mathrm{C}_{\mathrm{n}, \mathrm{k}}^{\nu}(0)\right|^{2}$} & \multicolumn{3}{|c|}{$\left|\mathrm{C}_{\mathrm{n}, \overline{\mathrm{k}}}^{\nu}(2)\right|^{2}$} & \\
\hline$\nu$ & 1 & 2 & 1 & & 2 & \\
\hline $\mathrm{n}^{\overline{\mathrm{k}}}$ & 0 & 0 & 0 & 2 & 0 & 2 \\
\hline 0 & 0.999 & - & 0.629 & 0.369 & 0.369 & 0.629 \\
\hline 1 & - & 0.962 & - & - & - & 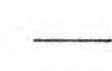 \\
\hline 2 & 0.001 & 0.029 & 0.001 & - & 0.001 & 0.001 \\
\hline \multicolumn{7}{|c|}{$\left|C_{n, k}^{\prime \prime}(4)\right|^{2}$} \\
\hline$\nu$ & 1 & & 2 & & & \\
\hline$n^{\bar{k}}$ & 0 & 2 & 0 & 2 & & \\
\hline 0 & 0.523 & 0.473 & 0.468 & 0.520 & & \\
\hline 1 & - & - & 0.001 & 0.001 & & \\
\hline 2 & 0.002 & 0.001 & 0.005 & 0.004 & & \\
\hline
\end{tabular}

Tab. 5.5. Probabilidade de encontrarmos o estado com $\mathrm{n}=0,1,2$ e $\overline{\mathrm{k}}=0,2$ na expansão (5.15) dos primeiros dois estados com $\mathrm{L}=0,2,4$ (base deformada com $\mathrm{N}=9$ ). 
(representação $2 \mathrm{p}-2 \mathrm{~b}$ )

\begin{tabular}{|c|c|c|c|c|c|c|c|c|}
\hline \multicolumn{5}{|c|}{ EXATO } & \multicolumn{4}{|c|}{ VÁCUO } \\
\hline $\mathrm{L}$ & \multicolumn{2}{|c|}{ E.EXC. $<r^{2}>^{1 / 2}$} & \multirow{2}{*}{$\begin{array}{l}\mathrm{BE} 2 \\
- \\
-\end{array}$} & \multirow{2}{*}{$\mathrm{Q}_{\mathrm{el}}$} & \multicolumn{2}{|c|}{ E.EXC. $<\mathrm{r}^{2}>^{1 / 2}$} & \multirow{2}{*}{$\begin{array}{l}\text { BE2 } \\
- \\
\end{array}$} & \multirow{2}{*}{$\mathrm{Q}_{\mathrm{el}}$} \\
\hline 0 & - & 2.99 & & & - & 2.99 & & \\
\hline 2 & 1.37 & 2.99 & 55.09 & -2.50 & 1.38 & 2.99 & 51.98 & -1.14 \\
\hline 4 & 4.54 & 2.98 & 82.91 & -12.87 & 4.59 & 2.97 & 80.17 & -12.43 \\
\hline \multicolumn{5}{|c|}{2 -fonons } & \multicolumn{4}{|c|}{3 -fonons } \\
\hline $\mathrm{L}$ & \multicolumn{2}{|c|}{ E.EXC. $<r^{2}>^{1 / 2}$} & $\mathrm{BE} 2$ & $\mathrm{Q}_{\mathrm{el}}$ & \multicolumn{2}{|c|}{ E.EXC. $<r^{2}>^{1 / 2}$} & $\mathrm{BE} 2$ & $\mathrm{Q}_{\mathrm{el}}$ \\
\hline 0 & - & 2.99 & - & - & - & 2.99 & - & - \\
\hline 2 & 1.37 & 2.99 & 56.16 & -3.10 & 1.37 & 2.99 & 55.73 & -2.78 \\
\hline 4 & 4.53 & 2.98 & 82.82 & -12.83 & 4.53 & 2.98 & 83.03 & -12.86 \\
\hline \multicolumn{5}{|c|}{ 4-fonons } & \multicolumn{4}{|c|}{ 5-fonons } \\
\hline L & E.EXC. & $r^{2}>^{1 / 2}$ & BE2 & $\mathrm{Q}_{\mathrm{el}}$ & E.EXC. & $r^{2}>^{1 / 2}$ & $\mathrm{BE} 2$ & $\mathrm{Q}_{\mathrm{el}}$ \\
\hline 0 & - & 2.99 & - & - & - & 2.99 & - & - \\
\hline 2 & 1.37 & 2.99 & 55.40 & -2.64 & 1.37 & 2.99 & 55.23 & -2.56 \\
\hline 4 & 4.54 & 2.98 & 83.00 & -12.87 & 4.54 & 2.98 & 82.96 & -12.87 \\
\hline
\end{tabular}

Tab. 5.6. Níveis de energia de excitação e propriedades geométricas dos estados de energias mais baixas para $\mathrm{L}=0,2,4$ calculados em uma base deformada truncada em $\mathrm{N}=0-5$. Energias em $\mathrm{MeV},\left\langle\mathrm{r}^{2}\right\rangle^{1 / 2}$ em fm, $\mathrm{BE} 2(\mathrm{~L} \rightarrow \mathrm{L}-2)$ em $\mathrm{e}^{2} \cdot \mathrm{fm}^{4}$ e $\mathrm{Q}_{\mathrm{el}}$ em e.fm². 


\subsubsection{Modelo $\mathrm{Sp}(1, \mathrm{R})$ Deformado Determinado pelo Estado de Peso Mínimo $4 \mathrm{p}-4 \mathrm{~b}$}

O cálculo que consideraremos a seguir, é equivalente ao realizado na seção 4.5 .3 utilizando uma base esférica. A partir dos estados de base esféricos axialmente simétricos $\mid \mathrm{n}(120), \mathrm{LM} 0 ; \mathrm{b}_{0}>$, que discutimos na seção 4.3.3, obtemos os estados deformados $\ln (120), L M 0 ; b_{\| \prime} b_{0}>$. Através do processo de Gram-Schimdt obtemos a base deformada ortogonalizada $\left|\mathrm{n}(120), \mathrm{LM} 0 ; \mathrm{b}_{0}\right\rangle_{\mathrm{GS}}$. Do mesmo modo que nas seções anteriores, os elementos de matriz dos operadores de interesse na base deformada são obtidos pela expansão desta, na base esférica truncada e por fim ortogonalizamos pelo processo de Gram-Schmidt. Usamos uma base esférica com $\mathrm{n}_{\text {máx }}=34$ o que assegurou que os elementos de matriz dos operadores de interesse não se alteravam significantemente para estados até 9-fonons.

$\mathrm{Na}$ tabela 5.7 mostramos os valores de $\mathrm{b}_{11}(\mathrm{~L})$, que minimizam o valor esperado da hamiltoniana no estado do vácuo deformado para $\mathrm{L}=0,2,4$. A função de onda obtida através da diagonalização da hamiltoniana em uma base com $\mathrm{N}$-fonons deformados é dada por:

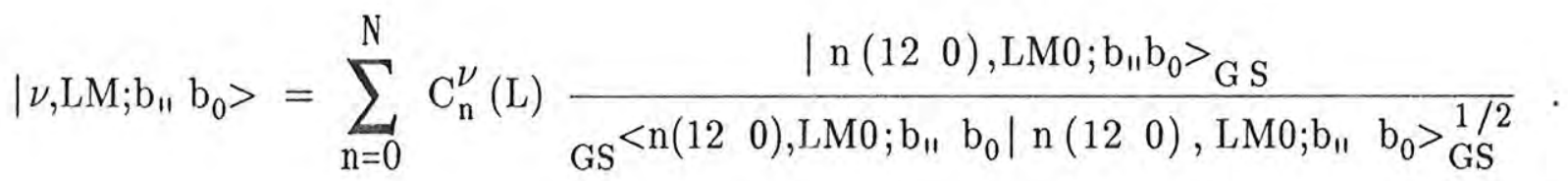

Na tabela 5.8 mostramos os valores de $\left|\mathrm{C}_{\mathrm{n}}^{\nu}(\mathrm{L})\right|^{2}$ que correspondem a probabilidade de encontrarmos nos dois estados de menor energia com um dado $\mathrm{L}$, o estado do vácuo, um e dois fonons deformados projetados em momento- angular $\mathrm{L}$ e ortogonalizados pelo processo de Gram-Schmidt. Na tabela 5.9 mostramos a energia de excitação e as propriedades geométricas dos estados de energia mais baixa com $\mathrm{L}=0,2,4$, obtidos 
através da diagonalização da hamiltoniana na base deformada truncada de $\mathrm{N}=0-5$. Na mesma tabela apresentamos o cálculo exato obtido no capítulo anterior.

Nas figuras 5.8-5.10 mostramos a contribuição parcial dos estados $\ln (120), \mathrm{LM} 0 ; \mathrm{b}_{\|} \mathrm{b}_{0}>_{\mathrm{GS}}$ ao momento de quadrupolo elétrico do primeiro estado $2^{+}$e ao $\mathrm{B}(\mathrm{E} 2,2 \rightarrow 0)$, entre os estados da banda fundamental e da primeira banda excitada. Estes gráficos são análogos a.os obtidos no capítulo anterior, 4.14-4.16.

A tabela 5.8 nos mostra que os primeiros dois estados de um dado L são dominados pela projeção em momento $\mathrm{L}$ do estado do vácuo e de 1-fonon deformado respectivamente, A contribuição da projeção do vácuo para o $\mathrm{B}\left(\mathrm{E} 2,2_{1}^{+} \longrightarrow 0_{1}^{+}\right)$e $\mathrm{Q}_{\mathrm{el}}\left(2_{1}^{+}\right)$ é maior do que $99 \%$.

Quando analisamos casos que envolvem estados excitados, como vemos na figura 5.10, notamos que mesmo nesses casos poucos estados deformados são necessários para descrevermos os operadores envolvidos. A partir da tabela 5.9 é evidente que a contribuição vinda de estados com mais de 5-fonons é desprezível. Se considerarmos apenas o estado do vácuo na base deformada, obtemos resultados que diferem em menos de $1 \%$ dos resultados exatos, sendo o BE2 o observável mais sensível.

Os resultados acima são qualitativamente os mesmos que obtivemos na seção 5.2 .1 e concluímos do mesmo modo, que os estados de mais baixa energia são muito bem descritos pela projeção em momento angular do vácuo do fonon deformado. 


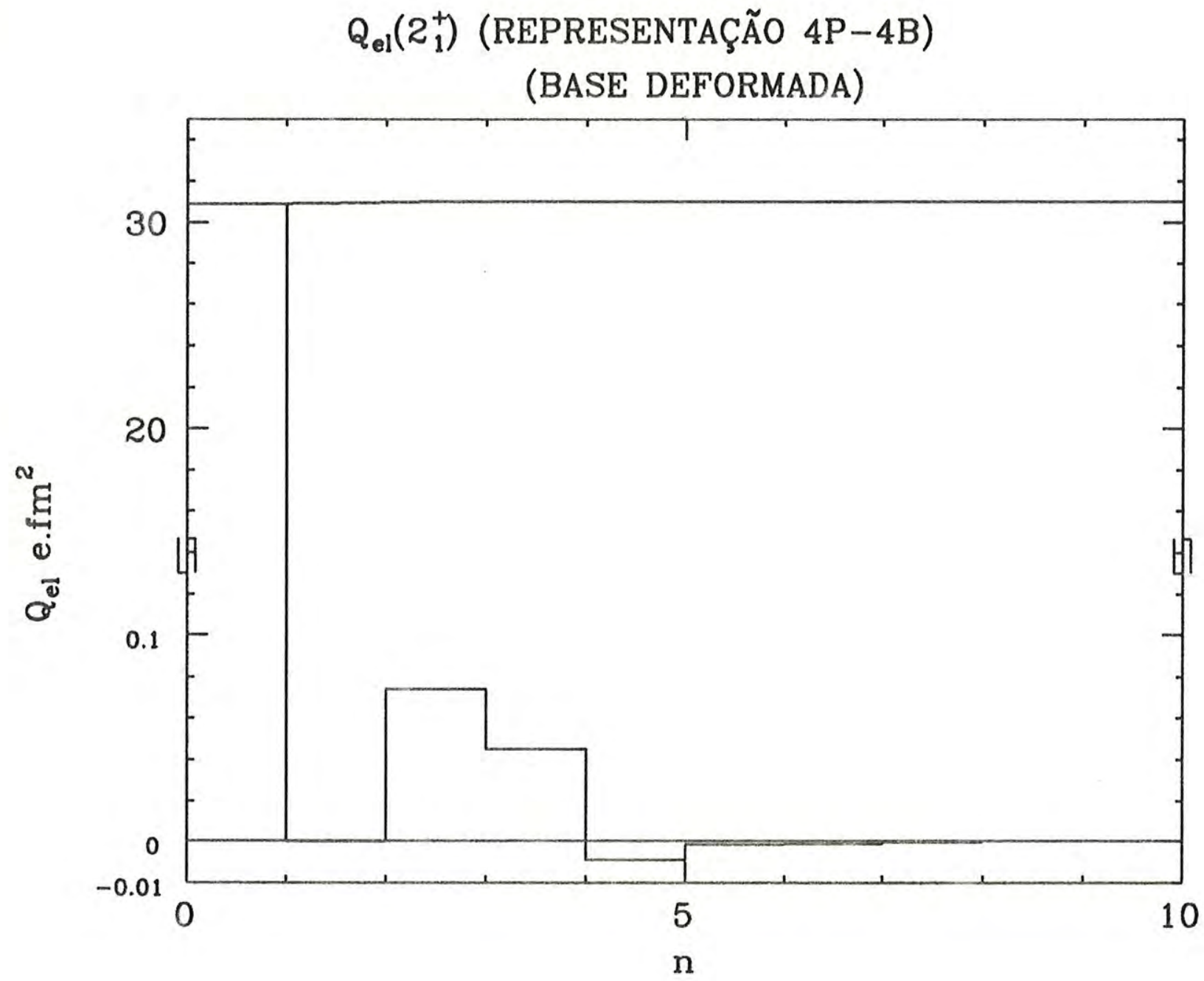

Fig. 5.8. Contribuição parcial ao $\mathrm{Q}_{\mathrm{el}}\left(2_{1}^{+}\right)$devido à inclusão sistemática, na expansão (5.16), dos estados de base do modelo $\mathrm{Sp}_{11}(1, \mathrm{R})$ deformado. O valor do $\mathrm{Q}_{\mathrm{el}}\left(2_{1}^{+}\right)$no ponto $\mathrm{n}$,é obtido considerando-se na expansão (5.16), a contribuição de todos os termos até $\mathrm{n}$, inclusive. Note a mudança de escala. 


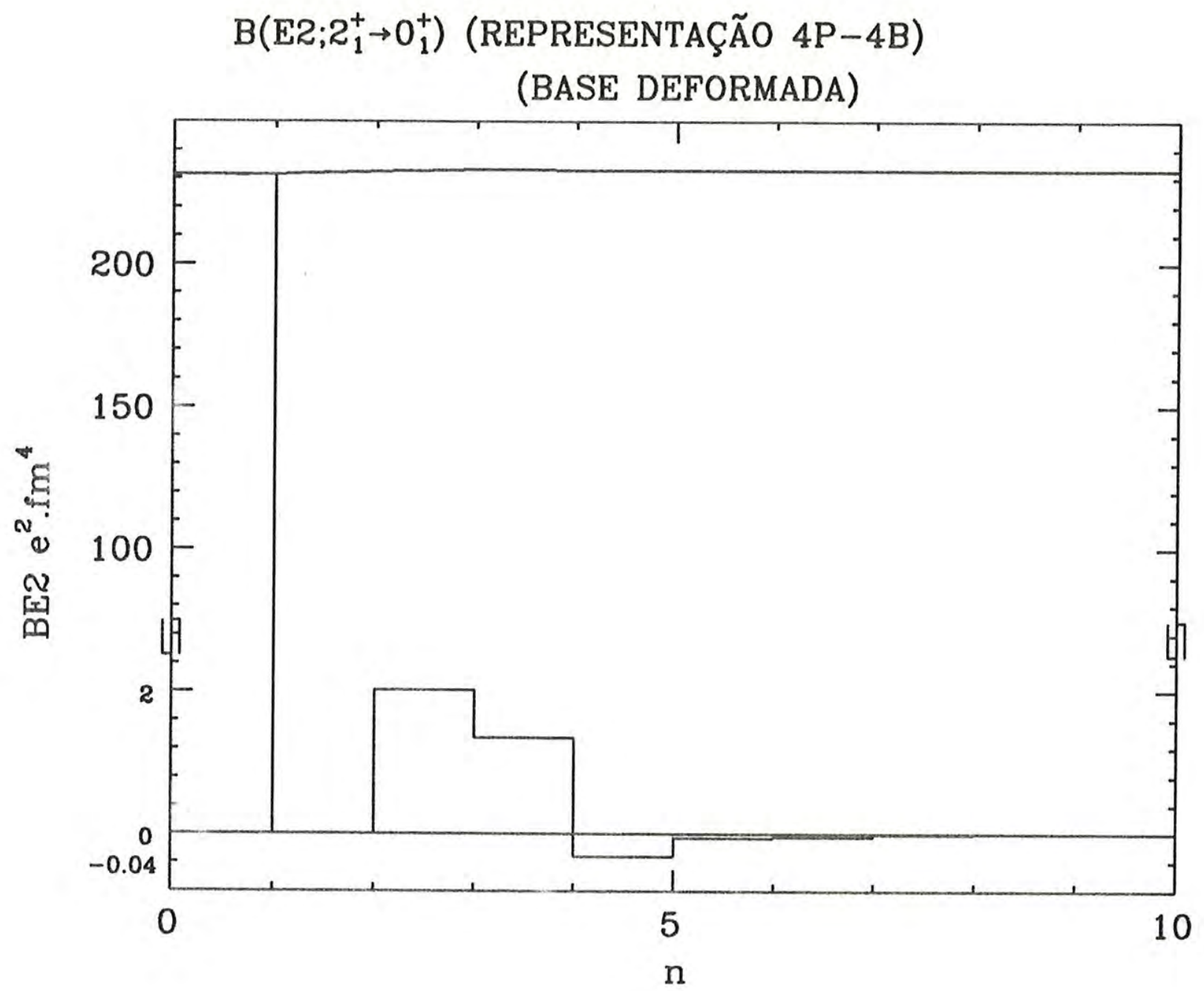

Fig. 5.9. Contribuição parcial ao $\mathrm{B}\left(\mathrm{E} 2,2_{1}^{+} \rightarrow 0_{1}^{+}\right)$, devido à inclusão sistemática na expansão (5.16) dos estados de base do modelo $\mathrm{Sp}_{11}(1, \mathrm{R})$ deformado. O valor do $\mathrm{B}(\mathrm{E} 2)$ no ponto $\mathrm{n}$,é obtido considerando-se na expansão (5.16), a contribuição de todos os termos até $\mathrm{n}$, inclusive. (Note a mudança de escala) 


\section{$\mathrm{B}\left(\mathrm{E} 2 ; 2_{2}^{+} \rightarrow \mathrm{O}_{2}^{+}\right)$(REPRESENTAÇÃO $4 \mathrm{P}-4 \mathrm{~B}$ ) \\ (BASE DEFORMADA)}

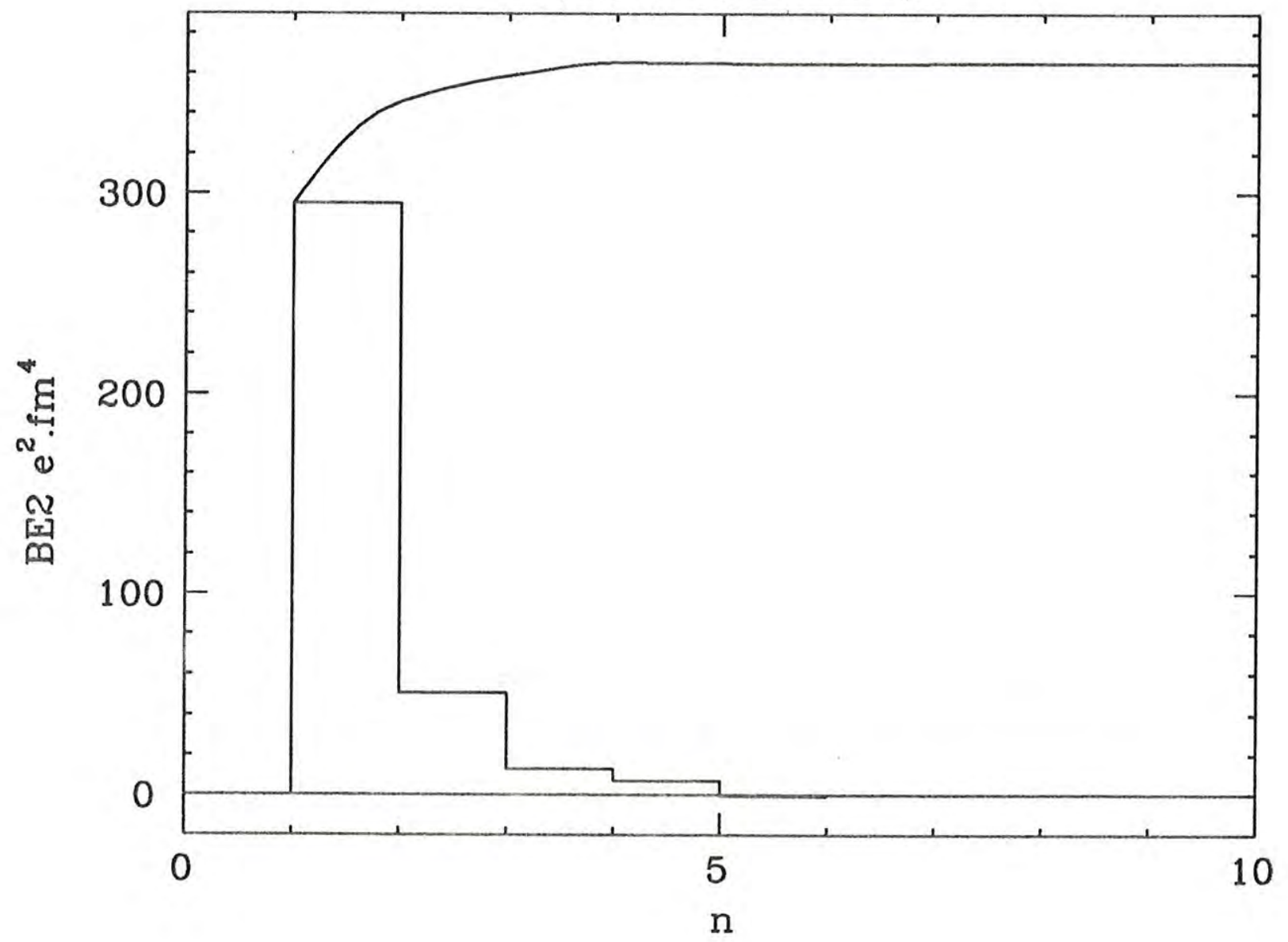

Fig. 5.10. Contribuição parcial ao $\mathrm{B}\left(\mathrm{E} 2,2_{2}^{+} \rightarrow 0_{2}^{+}\right)$, devido à inclusão sistemática na expansão (5.16) dos estados de base do modelo $\operatorname{Sp}_{11}(1, \mathrm{R})$ deformado. O valor do $\mathrm{B}(\mathrm{E} 2)$ no ponto $\mathrm{n}$, é obtido considerando-se na expansão (5.16), a contribuição de todos os termos até $n$, inclusive. 
(representação $4 \mathrm{p}-4 \mathrm{~b})$

\begin{tabular}{llll}
\hline & $\mathrm{L}=0$ & $\mathrm{~L}=2$ & $\mathrm{~L}=4$ \\
\hline $\mathrm{b}_{\text {II }}(\mathrm{fm})$ & 2.62 & 2.62 & 2.63 \\
\hline
\end{tabular}

Tab. 5.7. Valores de $b_{11}(L)$ que minimizam o valor esperado da hamiltoninana, no vácuo do fonon longitudinal deformado projetado em momento angular $\mathrm{L}\left(\mathrm{b}_{0}=1.45 \mathrm{fm}\right)$.

(representação $4 \mathrm{p}-4 \mathrm{~b}$ )

\begin{tabular}{ccccccc}
\hline & \multicolumn{2}{c}{$\left|\mathrm{C}_{\mathrm{n}}^{\nu}(0)\right|^{2}$} & \multicolumn{2}{c}{$\left|\mathrm{C}_{\mathrm{n}}^{\nu}(2)\right|^{2}$} & \multicolumn{2}{c}{$\left|\mathrm{C}_{\mathrm{n}}^{\nu}(4)\right|^{2}$} \\
\hline $\mathrm{n}^{\nu}$ & 0 & 1 & 0 & 1 & 0 & 1 \\
\hline 0 & 0.999 & - & 0.999 & - & 0.998 & - \\
1 & - & 0.969 & - & 0.969 & - & 0.967 \\
2 & 0.001 & 0.023 & 0.001 & 0.023 & 0.002 & 0.022 \\
\hline
\end{tabular}

Tab. 5.8. Probabilidade de encontrarmos o estado com $\mathrm{n}=0,1,2$ na expansão (5.16) dos dois estados de energias mais baixas com $\mathrm{L}=0,2,4$ (base deformada com $\mathrm{N}=5)$. 
(representação $4 \mathrm{p}-4 \mathrm{~b}$ )

\begin{tabular}{|c|c|c|c|c|c|c|c|c|}
\hline \multicolumn{5}{|c|}{ EXATO } & \multicolumn{4}{|c|}{ VÁCUO } \\
\hline $\mathrm{L}$ & E.EXC. < & $r^{2}>^{1 / 2}$ & $\mathrm{BE} 2$ & $\mathrm{Q}_{\mathrm{el}}$ & E.EXC. & $r^{2}>^{1 / 2}$ & $\mathrm{BE} 2$ & $\mathrm{Q}_{\mathrm{el}}$ \\
\hline 0 & - & 3.41 & - & - & - & 3.40 & - & - \\
\hline 2 & 1.04 & 3.41 & 232.85 & -31.00 & 1.04 & 3.41 & 231.39 & -30.89 \\
\hline 4 & 3.46 & 3.43 & 339.03 & -40.03 & 3.47 & 3.43 & 336.47 & -39.85 \\
\hline \multicolumn{5}{|c|}{2 -fonons } & \multicolumn{4}{|c|}{ 3-fonons } \\
\hline $\mathrm{L}$ & \multicolumn{2}{|c|}{ E.EXC. $<r^{2}>^{1 / 2}$} & $\mathrm{BE} 2$ & $\mathrm{Q}_{\mathrm{el}}$ & \multicolumn{2}{|c|}{ E.EXC. $<r^{2}>^{1 / 2}$} & $\mathrm{BE} 2$ & $\mathrm{Q}_{\mathrm{el}}$ \\
\hline 0 & - & 3.41 & - & - & - & 3.41 & - & - \\
\hline 2 & 1.03 & 3.41 & 232.40 & -30.97 & 1.04 & 3.41 & 233.07 & -31.01 \\
\hline 4 & 3.45 & 3.43 & 338.24 & -40.00 & 3.46 & 3.43 & 339.21 & -40.03 \\
\hline \multicolumn{5}{|c|}{ 4-fonons } & \multicolumn{4}{|c|}{ 5-fonons } \\
\hline $\mathrm{L}$ & E.EXC. < & $r^{2}>^{1 / 2}$ & $\mathrm{BE} 2$ & $\mathrm{Q}_{\mathrm{el}}$ & E.EXC. & $r^{2}>^{1 / 2}$ & $\mathrm{BE} 2$ & $\mathrm{Q}_{\mathrm{el}}$ \\
\hline 0 & - & 3.41 & - & - & - & 3.41 & - & - \\
\hline 2 & 1.04 & 3.41 & 232.91 & -31.00 & 1.04 & 3.41 & 232.88 & -31.00 \\
\hline 4 & 3.46 & 3.43 & 339.07 & -40.03 & 3.46 & 3.43 & 339.05 & -40.03 \\
\hline
\end{tabular}

Tab. 5.9. Níveis de energia de excitação e propriedades geométricas dos estados da banda rotacional fundamental calculados em uma base deformada truncada em $\mathrm{N}=0-5$. Energias em $\mathrm{MeV},\left\langle\mathrm{r}^{2}>^{1 / 2}\right.$ em $\mathrm{fm}, \mathrm{BE} 2(\mathrm{~L} \rightarrow \mathrm{L}-2)$ em $\mathrm{e}^{2} \cdot \mathrm{fm}^{4}$ e $\mathrm{Q}_{\mathrm{el}}$ em $\mathrm{e} \cdot \mathrm{fm}^{2}$. 


\section{Efeito da Mistura de Representações Irredutf́veis de $\mathrm{Sp}(1, \mathrm{R})$ 6.1. Introdução}

Neste capítulo vamos mostrar que um cálculo envolvendo a mistura de representações irredutíveis de $S p(1, R)$ é factível. A nossa inteção é realizar apenas um cálculo exploratório envolvendo o acoplamento dos estados do modelos $\mathrm{Sp}(1, \mathrm{R})$ que consideramos no capítulo 4. Como vimos, tais modelos são obtidos a partir dos estados de peso mínimo $0 \mathrm{p}-0 \mathrm{~b}, 2 \mathrm{p}-2 \mathrm{~b}$ e $4 \mathrm{p}-4 \mathrm{~b}$.

A necessidade de considerarmos a mistura de representações irredutíveis de $\mathrm{Sp}(3, \mathrm{R})$, surge de modo natural quando procuramos descrever as propriedades do espectro de baixa energia do ${ }^{12} \mathrm{C}$ como veremos a seguir.

\subsection{Níveis de Baixa Energia do ${ }^{12} \mathrm{C}$ e a Mistura de Representações Irredutíveis}

Arickx et al, na ref.(4) mostraram que a banda rotacional fundamental pode ser descrita pelo modelo $\mathrm{Sp}_{\perp}(1, \mathrm{R})$, obtido a partir do estado de peso mínimo 0p-0b , eq(3.5), que reproduzimos abaixo:

$$
\left|0(04) ; \mathrm{b}_{\perp}\right\rangle^{\mathrm{e}}=\frac{1}{\sqrt{12} !} \operatorname{det}(000)^{4}(010)^{4}(001)^{4} .
$$

Através de um cálculo do tipo PAV, Bouten et al, ref.(15), mostraram que o estado $0_{2}^{+}$, observado a uma energia de excitação de $7.65 \mathrm{MeV}^{(12)}$, podia ser considerado um estado intruso associado a uma configuração prolata com rótulos de Elliott (12 0). No capítulo 5 mostramos que o modelo $\mathrm{Sp}(1, \mathrm{R})$ podia ser considerado como uma generalização do 
método PAV. Assim, no contexto do modelo $\mathrm{Sp}(1, \mathrm{R})$, este primeiro estado $0^{+}$excitado, é descrito pelo modelo $\mathrm{Sp}_{11}(1, \mathrm{R})$, obtido a partir do estado de peso mínimo $4 \mathrm{p}-4 \mathrm{~b}$, eq(3.20) que reproduzimos abaixo:

$$
\mid 0(120) ; b_{11}>^{e}=\frac{1}{\sqrt{12} !} \operatorname{det}(000)^{4}(100)^{4}(200)^{4}
$$

portanto é necessário considerarmos as representações irredutíveis $0 \mathrm{p}-0 \mathrm{~b}$ e $4 \mathrm{p}-4 \mathrm{~b}$ para descrevermos o espectro de baixa energia ${ }^{12} \mathrm{C}$.

$\mathrm{O}$ espectro de baixa energia experimental do ${ }^{12} \mathrm{C}$, relevante para a análise que faremos a seguir é apresentado no lado esquerdo da figura 6.1. Na mesma figura mostramos os resultados experimentais obtidos para o elemento de matriz de transição monopolar elétrica e B(E2) entre os estados considerados. Por definição o elemento de matriz de transição monopolar elétrica entre os estados $\left|0_{2}^{+}\right\rangle$e $\left|0_{1}^{+}\right\rangle$é dado por:

$$
\mathrm{M}\left(0_{2}^{+} \rightarrow 0_{1}^{+}\right)=\left\langle 0_{1}^{+}\left|\mathrm{M}_{\mathrm{el}}\right| 0_{2}^{+}\right\rangle
$$

Como discutimos acima, no contexto do modelo $\mathrm{Sp}(1, \mathrm{R})$, associamos a banda rotacional fundamental do ${ }^{12} \mathrm{C}$ a representação $0 \mathrm{p}-0 \mathrm{~b}$ de $\mathrm{Sp}_{\perp}(1, \mathrm{R})$ e o estado $0_{2}^{+}$a representação irredutível $4 \mathrm{p}-4 \mathrm{~b}$ de $\mathrm{Sp}_{\|}(1, \mathrm{R})$.

Os operadores momento de monopolo e quadrupolo, como vimos no capítulo 2, pertencem à álgebra $\mathrm{sp}(3, \mathrm{R})$ e portanto transições monopolares e quadrupolares entre representações irredutíveis distintas de $\mathrm{Sp}(3, \mathrm{R})$ são proibidas do ponto de vista do modelo simplético. Assim, transições monopolares e quadrupolares entre os estados da banda rotacional fundamental e o estado $0_{2}^{+}$do ${ }^{12} \mathrm{C}$, são proibidas por construção no modelo 
$\mathrm{Sp}(1, \mathrm{R})$. No entanto, por exemplo, notamos na figura 6.1 que experimentalmente são observadas a transição monopolar entre os estados $0_{2}^{+}$e $0_{1}^{+}$e a quadrupolar entre $0_{2}^{+}$e $2_{1}^{+}$. Assim, concluímos que é essencial considerarmos o acoplamento entre as representações $0 \mathrm{p}-0 \mathrm{~b}$ e $4 \mathrm{p}-4 \mathrm{~b}$ de $\mathrm{Sp}(1, \mathrm{R})$, para descrevermos as transições eletromagnéticas através do modelo simplético.

O procedimento mais natural a seguir, consiste em acoplar diretamente as representações $0 \mathrm{p}-0 \mathrm{~b}$ e $4 \mathrm{p}-4 \mathrm{~b}$ através da interação $\mathrm{V}$ de dois corpos que não pertence à álgebra $\mathrm{sp}(3, \mathrm{R})$. Notemos que a energia cinética também pertence à álgebra $\mathrm{sp}(3, \mathrm{R}) \mathrm{e}$ portanto não vai acoplar representações diferentes. A seguir analisaremos tal procedimento.

\subsection{Cálculo do Acoplamento entre Representações Irredutíveis de $\operatorname{Sp}(1, \mathrm{R})$ através do MFG}

Para calcularmos os elementos de matriz do potencial entre os estados de base dos modelos $\mathrm{Sp}(1, \mathrm{R})$ associados aos estados de peso mínimo $0 \mathrm{p}-0 \mathrm{~b}$ e $4 \mathrm{p}-4 \mathrm{~b}$ vamos utilizar o método da função geratriz, que apresentamos no capítulo 4. Nesse capítulo consideramos o MFG, no caso em que tínhamos apenas uma única representação irredutível de $\operatorname{Sp}(1, R)$. No entanto, a sua generalização para a situação onde calculamos o acoplamento entre representações irredutíveis distintas de $S p(1, R)$ é trivial e portanto, apresentaremos diretamente os resultados.

\subsubsection{Acoplamento entre as Representações $0 p-0 b$ e $4 p-4 b$}

Os elementos de matriz do potencial entre os estados de base do modelo $\mathrm{Sp}_{\perp}(1, \mathrm{R})$ associado ao estado de peso mínimo $0 \mathrm{p}-0 \mathrm{~b}$ e do modelo $\mathrm{Sp}_{\|}(1, \mathrm{R})$ associado ao estado de 
peso mínimo $4 \mathrm{p}-4 \mathrm{~b}$, são obtidos através do MFG pela seguinte expressão:

$$
\begin{aligned}
& <\mathrm{n}^{\prime}(04) \mathrm{LM} 0 ; \mathrm{b}_{\perp}|\mathrm{V}| \mathrm{n}(120), \mathrm{LM} 0 ; \mathrm{b}_{11}>=\frac{(2 \mathrm{~L}+1)}{2} \int_{-1}^{1} \mathrm{dx} \mathrm{P}_{\mathrm{L}}(\mathrm{x}) \cdot \\
& \cdot \frac{\Gamma(19)^{1 / 2} \Gamma(35 / 2)^{1 / 2}}{\left(\Gamma\left(\mathrm{n}^{\prime}+1\right) \Gamma(\mathrm{n}+1) \Gamma\left(\mathrm{n}^{\prime}+19\right) \Gamma(\mathrm{n}+35 / 2)\right)^{1 / 2}} \frac{\partial^{\mathrm{n}^{\prime}}}{\partial \tilde{\beta}_{\perp}^{\mathrm{n}^{\prime}}} \frac{\partial^{\mathrm{n}}}{\partial \tilde{\beta}_{\| 1}^{\mathrm{n}}} \cdot \\
& \cdot<0(04) ; \mathrm{b}_{\perp}\left|\mathrm{e}_{\perp}^{\tilde{\beta}_{\perp} \mathrm{A}_{\perp}\left(\mathrm{b}_{\perp}\right)} \mathrm{VR}_{\mathrm{y}}(\beta) \mathrm{e}^{\tilde{\beta}_{\| 1} \mathrm{~A}_{\|}^{+}\left(\mathrm{b}_{11}\right)}\right| 0(120) ; \mathrm{b}_{11}>\mid \begin{array}{l}
\tilde{\beta}_{\perp}=0 \\
\tilde{\beta}_{11}=0
\end{array}
\end{aligned}
$$

e a partir das equações (4.35) e (4.36) é imeditao que:

$$
\begin{aligned}
& <0(04) ; \mathrm{b}_{\perp}\left|\mathrm{e}^{\tilde{\beta}_{\perp} \mathrm{A}_{\perp}\left(\mathrm{b}_{\perp}\right)} \mathrm{VR} \mathrm{R}_{\mathrm{y}}(\beta) \mathrm{e}^{\tilde{\beta}_{11} \mathrm{~A}_{11}^{+}\left(\mathrm{b}_{11}\right)}\right| 0(120) ; \mathrm{b}_{11}>=
\end{aligned}
$$

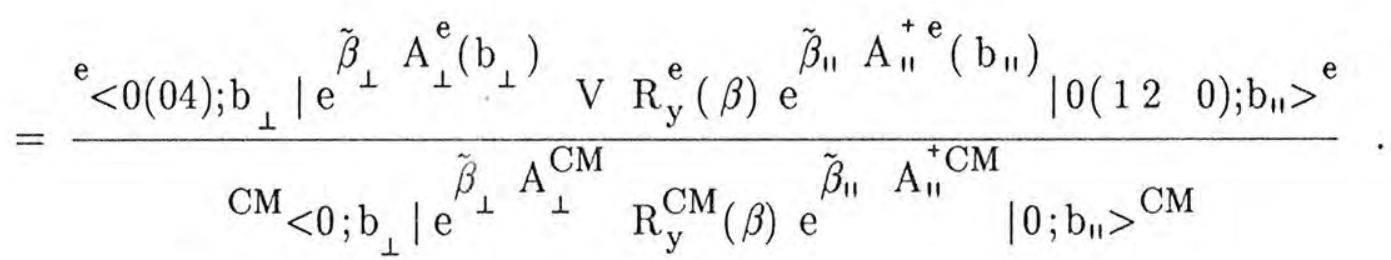

Fazendo uma análise semelhante a que fizemos no capítulo 4, notamos que o numerador da expressão acima envolve o cálculo do operador de dois corpos V entre estados determinantais, e como $\mathrm{V}$ não depende de spin-isospin, obtemos: 


$$
\begin{aligned}
& { }^{\mathrm{e}}<0(04) ; \mathrm{b}_{\perp}\left|\mathrm{e}^{\tilde{\beta}_{\perp} \mathrm{A}_{\perp}^{\mathrm{e}}\left(\mathrm{b}_{\perp}\right)} \mathrm{VR}_{\mathrm{y}}^{\mathrm{e}}(\beta) \mathrm{e}^{\tilde{\beta}_{11} \mathrm{~A}_{11}^{+\mathrm{e}}\left(\mathrm{b}_{11}\right)}\right| 0(120) ; \mathrm{b}_{11}>^{\mathrm{e}}= \\
& =\frac{1}{2} \sum_{\substack{\mathrm{i} j \mathrm{k} 1 \\
\mathrm{ab}}}<\phi_{\mathrm{i} ; \mathrm{a}} \phi_{\mathrm{j} ; \mathrm{b}}\left|\mathrm{V}_{12}\right| \tilde{\phi}_{\mathrm{k} ; \mathrm{a}} \tilde{\phi}_{1 ; \mathrm{b}}>\mathrm{C}_{\text {ia jb ka lb }}
\end{aligned}
$$

onde omitimos o comprimento do oscilador na notação relativa aos operadores da álgebra $\mathrm{sp}(1, \mathrm{R})$. Sempre que não causar confusão, vamos fazer isto para simplificar a notação.

As funções de onda de partícula independente $\phi_{\mathrm{i} ; \mathrm{a}}$ e $\tilde{\phi}_{\mathrm{j} ; \mathrm{b}}$ na eq.(4), são associadas respectivamente aos estados determinantais:

$$
\begin{aligned}
& |\phi\rangle=\mathrm{e}^{\tilde{\beta}_{\perp} \mathrm{A}_{\perp}^{+\mathrm{e}}\left(\mathrm{b}_{\perp}\right)} \mid 0(04) ; \mathrm{b}_{\perp}>^{\mathrm{e}} \\
& |\tilde{\phi}\rangle=\mathrm{R}_{\mathrm{y}}^{\mathrm{e}(\beta)} \mathrm{e}^{\tilde{\beta}_{11} \mathrm{~A}_{11}^{+\mathrm{e}\left(\mathrm{b}_{11}\right)} \mid 0(120) ; \mathrm{b}_{11}>^{\mathrm{e}} .}
\end{aligned}
$$

O duplo-cofator envolvido na equação (4), é obtido a partir da matriz de overlap $\mathrm{B}_{\alpha \beta}=\left\langle\phi_{\alpha}\right| \tilde{\phi}_{\beta}>$, que tem os elementos de matriz seguintes:

$$
\mathrm{B}_{\mathrm{i}, \mathrm{j} \mathrm{b}}=\left\langle\phi_{\mathrm{i}}\left(\mathrm{b}_{\perp}\right)\left|\mathrm{e}^{\tilde{\beta}_{\perp} \mathrm{A}_{\perp}^{\mathrm{e}}\left(\mathrm{b}_{\perp}\right)} \mathrm{R}_{\mathrm{y}}^{\mathrm{e}}(\beta) \mathrm{e}^{\tilde{\beta}_{11} \mathrm{~A}_{11}^{+\mathrm{e}}\left(\mathrm{b}_{11}\right)}\right| \phi_{\mathrm{j}}\left(\mathrm{b}_{11}\right)>\delta_{\mathrm{ab}}\right.
$$

das equações (1) e (2), segue que o índice "i" indica a ocupação das funções de onda (000), (010) e (001) do oscilador harmônico na base cartesiana com comprimento $b_{\perp}$ e "j" indica as funções de onda (000), (100) e (200) do oscilador com comprimento b Definindo a notação mais concisa: 


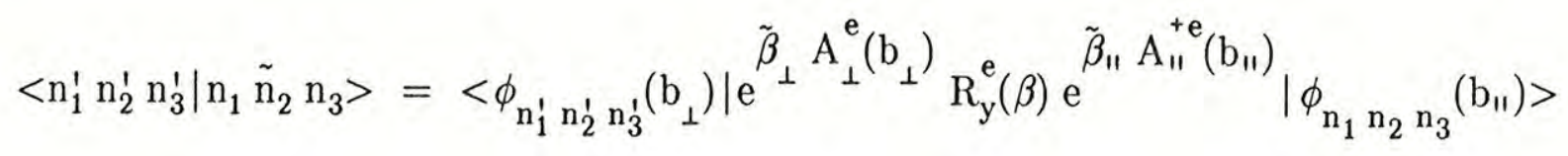

podemos escrever a matriz $\mathrm{B}$ da seguinte maneira:

$$
\mathrm{B}=\left[\begin{array}{rrrrr}
\mathrm{B}_{12}^{\mathrm{n}_{+}} & & & \\
& & & 0 \\
& \mathrm{~B}_{12}^{\mathrm{n}-} & & \\
& & \mathrm{B}_{12}^{\mathrm{p+}} & \\
& & & \mathrm{B}_{12}^{\mathrm{p}-} \\
0 & & & & \\
& & & &
\end{array}\right]
$$

com

$$
\begin{gathered}
\mathrm{B}_{12}=\left[\begin{array}{ccc}
<000 \mid 0 \tilde{0} 0> & <000 \mid 1 \tilde{0} 0> & <000 \mid 2 \tilde{0} 0> \\
<010 \mid 0 \tilde{0} 0> & <010 \mid 1 \tilde{0} 0> & <010 \mid 2 \tilde{0} 0> \\
<001 \mid 0 \tilde{0} 0> & <001 \mid 1 \tilde{0} 0> & <001 \mid 2 \tilde{0} 0>
\end{array}\right]= \\
=\left[\begin{array}{ccc}
<000 \mid 0 \tilde{0} 0> & 0 & <000 \mid 2 \tilde{0} 0> \\
0 & <010 \mid 1 \tilde{0} 0> & 0 \\
0 & <001 \mid 100> & 0
\end{array}\right] .
\end{gathered}
$$

A igualdade acima é obtida notando-se que vários termos se anulam devido à paridade. $\mathrm{Na}$ eq.(6) os índices superiores indicam o estado de spin-isospin ocupado pelas funções de onda espaciais associadas aos blocos $\mathrm{B}_{12}$ correspondentes.

$\mathrm{O}$ cálculo direto resulta que $\operatorname{det} \mathrm{B}_{12}=0$ e como discutimos detalhadamente no apêndice 1, concluímos que não existe duplo cofator da forma dada na eq.(4) não nulo. Do 
ponto de vista físico, isto significa que não existe interação de dois corpos $\mathrm{V}$, da forma dada na seção 4.4.3, que acople diretamente os estados dos modelos $\operatorname{Sp}_{\perp}(1, R)$ e $\operatorname{Sp}_{11}(1, R)$ associados aos estados de peso mínimo $0 \mathrm{p}-0 \mathrm{~b}$ e $4 \mathrm{p}-4 \mathrm{~b}$ respectivamente. Portanto só podemos acoplar as representações irredutíveis $0 \mathrm{p}-0 \mathrm{~b}$ e $4 \mathrm{p}-4 \mathrm{~b}$ indiretamente, a partir da inclusão de uma terceira representação irredutível de $\mathrm{Sp}(1, \mathrm{R})$.

Para escolhermos esta terceira representação irredutível, vamos nos basear no trabalho de Bouten et al ${ }^{(15)}$, que através de um cálculo do tipo PAV, procura descrever o possível estado $0_{3}^{+}$a uma energia de excitação de $10.3 \mathrm{MeV}$, a partir do estado de peso mínimo de $\mathrm{SU}(3)$ triaxial, com rótulos de Elliott (62). No contexto do modelo $\mathrm{Sp}(1, \mathrm{R})$, uma vez que $(\lambda \mu)=(62)$ tem $\lambda>\mu$ vamos considerar o modelo $\operatorname{Sp}_{11}(1, \mathrm{R})$ obtido a partir do estado de peso mínimo 2p-2b, eq.(4.13). Vamos a seguir mostrar que a representação $2 \mathrm{p}-2 \mathrm{~b}$ se acopla à representação $0 \mathrm{p}-0 \mathrm{~b}$.

\subsubsection{Acoplamento entre as Representações $0 \mathrm{p}-0 \mathrm{~b}$ e $2 \mathrm{p}-2 \mathrm{~b}$}

Os elementos de matriz do potencial, entre os estados de base do modelo $\mathrm{Sp}_{\perp}(1, \mathrm{R})$ associado ao estado de peso mínimo $0 \mathrm{p}-0 \mathrm{~b}$ e do modelo $\mathrm{Sp}_{\|}(1, \mathrm{R})$ associado ao estado de peso mínimo $2 \mathrm{p}-2 \mathrm{~b}$, são obtidos através do MFG, pela seguinte expressão:

$$
\begin{aligned}
& <\mathrm{n}^{\prime}(04), \mathrm{LM} 0 ; \mathrm{b}_{\perp}|\mathrm{V}| \mathrm{n}(62), \mathrm{LMK} ; \mathrm{b}_{\|}>=\frac{(2 \mathrm{~L}+1)}{4} \int_{-1}^{1} \mathrm{dx} \mathrm{d}_{0 \mathrm{~K}}^{\mathrm{L}}(\mathrm{x}) \cdot \\
& \cdot \frac{\Gamma(19)^{1 / 2} \Gamma(27 / 2)^{1 / 2}}{\left(\Gamma\left(\mathrm{n}^{\prime}+1\right) \Gamma(\mathrm{n}+1) \Gamma\left(\mathrm{n}^{\prime}+19\right) \Gamma(\mathrm{n}+27 / 2)\right)^{1 / 2}} \frac{\partial^{\mathrm{n}^{\prime}}}{\partial \tilde{\beta}_{\perp}^{\mathrm{n}^{\prime}}} \frac{\partial^{\mathrm{n}}}{\partial \tilde{\beta}_{\| \prime}^{\mathrm{n}}} \cdot
\end{aligned}
$$




$$
\cdot<0(04) ; \mathrm{b}_{\perp}\left|\mathrm{e}^{\tilde{\beta}_{\perp} \mathrm{A}_{\perp}\left(\mathrm{b}_{\perp}\right)} \mathrm{VR} \mathrm{R}_{\mathrm{y}}(\beta) \mathrm{R}_{\mathrm{z}}(\gamma) \mathrm{e}^{\tilde{\beta}_{11} \mathrm{~A}_{11}^{+}\left(\mathrm{b}_{11}\right)}\right| 0(62) ; \mathrm{b}_{11}>\mid \begin{aligned}
& \tilde{\beta}_{\perp}=0 \\
& \tilde{\beta}_{11}=0
\end{aligned}
$$

e a partir das equações (4.35) e (4.36) é imediato que:

$$
\begin{aligned}
& <0(04) ; \mathrm{b}_{\perp}\left|\mathrm{e}^{\tilde{\beta}_{\perp} \mathrm{A}_{\perp}\left(\mathrm{b}_{\perp}\right)} \mathrm{V} \mathrm{R}_{\mathrm{y}}(\beta) \mathrm{R}_{\mathrm{z}}(\gamma) \mathrm{e}^{\tilde{\beta}_{11} \mathrm{~A}_{11}^{+}\left(\mathrm{b}_{11}\right)}\right| 0(62) ; \mathrm{b}_{11}>= \\
& =\frac{e^{\mathrm{e}}<0(04) ; \mathrm{b}_{\perp}\left|\mathrm{e}^{\tilde{\beta}_{\perp} \mathrm{A}_{\perp}^{\mathrm{e}}\left(\mathrm{b}_{\perp}\right)} \mathrm{VR} \mathrm{R}_{\mathrm{y}}^{\mathrm{e}}(\beta) \mathrm{R}_{\mathrm{z}}^{\mathrm{e}}(\gamma) \mathrm{e}^{\tilde{\beta}_{11} \mathrm{~A}_{11}^{+\mathrm{e}}\left(\mathrm{b}_{11}\right)}\right| 0(62) ; \mathrm{b}_{11}>}{\mathrm{e}} .
\end{aligned}
$$

O estado de peso mínimo da representação $2 \mathrm{p}-2 \mathrm{~b}$, eq.(4.13), consiste em uma soma de seis determinantes de Slater. Portanto o numerador da expressão acima é dado por seis termos da forma dada na eq.(6.4), onde os duplo-cofatores envolvidos no caso em questão e as respectivas funções onda de partícula independente são obtidas a partir do overlap entre o estado $\left|0(04) ; \mathrm{b}_{\perp}\right\rangle^{\mathrm{e}}$, eq.(6.1) e os seis determinantes $\left|\psi^{\{\mathrm{C}\}_{;}} \mathrm{b}_{11}\right\rangle^{\mathrm{e}}$, que formam o estado $\mid 0(62) ; b_{\|}>^{e}$, eq.(4.13).

Analisando as seis matrizes de overlap $\mathrm{B}^{\{\mathrm{C}\}}={ }^{\mathrm{e}}<0(04) ; \mathrm{b}_{\perp} \mid \psi^{\{\mathrm{C}\}} ; \mathrm{b}_{11}>{ }^{\mathrm{e}}$ citadas acima, podemos mostrar que elas diferem entre si apenas na ocupação dos estados de spin-isospin dos blocos associados ao overlap entre as funções de onda espaciais e como $\mathrm{V}$ não depende do spin e do isospin, concluímos que os seis termos envolvidos no numerador da eq.(6.7) contribuem de modo idêntico. Assim sem perda de generalidade podemos escrever: 


$$
\begin{aligned}
& { }^{e}<0(04) ; \mathrm{b}_{\perp}\left|\mathrm{e}^{\tilde{\beta}_{\perp} \mathrm{A}_{\perp}^{\mathrm{e}}} \mathrm{VR}_{\mathrm{y}}^{\mathrm{e}}(\beta) \mathrm{R}_{\mathrm{z}}^{\mathrm{e}}(\gamma) \mathrm{e}^{\tilde{\beta}_{11} \mathrm{~A}_{11}^{+\mathrm{e}}}\right| 0(62) ; \mathrm{b}_{\mathrm{H}}>^{\mathrm{e}}= \\
& =\sqrt{6} \sum_{\substack{\mathrm{i} j \mathrm{k} 1 \\
\mathrm{ab} \\
\mathrm{ia}<\mathrm{jb}}}<\phi_{\mathrm{i} ; \mathrm{a}} \phi_{\mathrm{j} ; \mathrm{b}}\left|\mathrm{V}_{12}\right| \tilde{\phi}_{\mathrm{k} ; \mathrm{a}} \tilde{\phi}_{\mathrm{l} ; \mathrm{b}}>\mathrm{C}_{\mathrm{ia} \text { jb ka lb }}^{\{1122\}}
\end{aligned}
$$

onde $\mathrm{C}_{\mathrm{ia} \text { jb ka lb }}^{\{1122\}}$ é o duplo cofator obtido a partir da matriz de overlap abaixo:

$$
\mathrm{B}^{\{1122\}}=\left[\begin{array}{cccc}
\mathrm{B}_{11}^{\mathrm{n}+} & & & \\
& \mathrm{B}_{11}^{\mathrm{n}-} & & \\
& & \mathrm{B}_{12}^{\mathrm{p}_{+}} & \\
& & & \\
& & & \mathrm{B}_{12}^{\mathrm{p}-}
\end{array}\right]
$$

onde

$$
\mathrm{B}_{11}=\left[\begin{array}{ccc}
<000 \mid 00 \tilde{0}> & 0 & 0 \\
0 & <010 \mid 100> & <010 \mid 0 \tilde{1} 0> \\
0 & 0 & <001 \mid 0 \tilde{1} 0>
\end{array}\right]
$$

e

$$
\mathrm{B}_{12}=\left[\begin{array}{ccc}
<000 \mid 0 \tilde{0} 0> & 0 & <000 \mid 2 \tilde{0} 0> \\
0 & <010 \mid 10 \tilde{0} 0> & 0 \\
0 & 0 & 0
\end{array}\right]
$$

a notação relativa aos elementos de matriz acima é definida abaixo: 


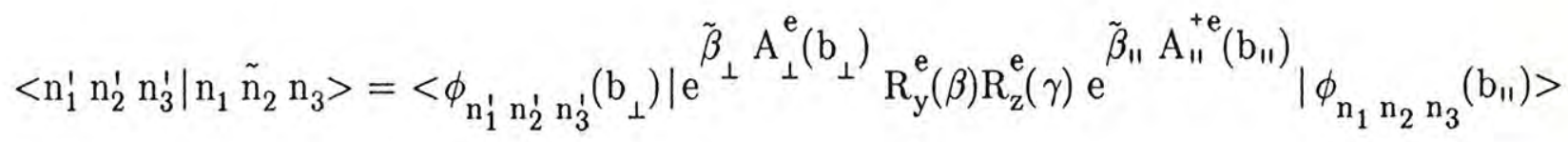

consideramos em (6.11) que vários termos se anulam devido à paridade. Através do uso das técnicas descritas nos apêndices 1 e 2, obtemos os elementos de matriz envolvidos nas matrizes $B_{11}$ e $B_{12}$ acima, que resultam ser todos não nulos.

Calculando diretamente obtemos que $\operatorname{det} B_{11} \neq 0$ e $\operatorname{det} B_{12}=0$. Uma vez que $\operatorname{det} B_{11} \neq 0$ e det $B_{12}=0$, necessariamente os duplo-cofatores não nulos, envolvidos na eq.(6.9), devem ter índices envolvendo as linhas e colunas das matrizes $B_{12}$, na eq.(6.10). Podemos facilmente mostrar que existem quatro duplo cofatores não nulos que irão contribuir na eq.(6.9) e portanto ocorre o acoplamento entre as representações $0 \mathrm{p}-0 \mathrm{~b}$ e $2 \mathrm{p}-2 \mathrm{~b}$.

\subsubsection{Acoplamento entre as Representações $2 \mathrm{p}-2 \mathrm{~b}$ e $4 \mathrm{p}-4 \mathrm{~b}$}

Devido à grande analogia entre esta seção e a anterior vamos ser mais concisos. Para obtermos os elementos de matriz do potencial entre os estados de base das representações irredutíveis $2 \mathrm{p}-2 \mathrm{~b}$ e $4 \mathrm{p}-4 \mathrm{~b}$ através do MFG precisamos calcular a seguinte expressão:

$$
\begin{aligned}
& <0(62) ; \mathrm{b}_{11}\left|\mathrm{e}^{\tilde{\beta}_{11}^{\prime} \mathrm{A}_{\| 1}\left(\mathrm{~b}_{11}\right)} \mathrm{V} \mathrm{R} \mathrm{R}_{\mathrm{z}}(\alpha) \mathrm{R}_{\mathrm{y}}(\beta) \mathrm{e}^{\tilde{\beta}_{\| 1} \mathrm{~A}_{\| 1}^{+}\left(\mathrm{b}_{112}\right)}\right| 0(120) ; \mathrm{b}_{\|_{2}}>=
\end{aligned}
$$

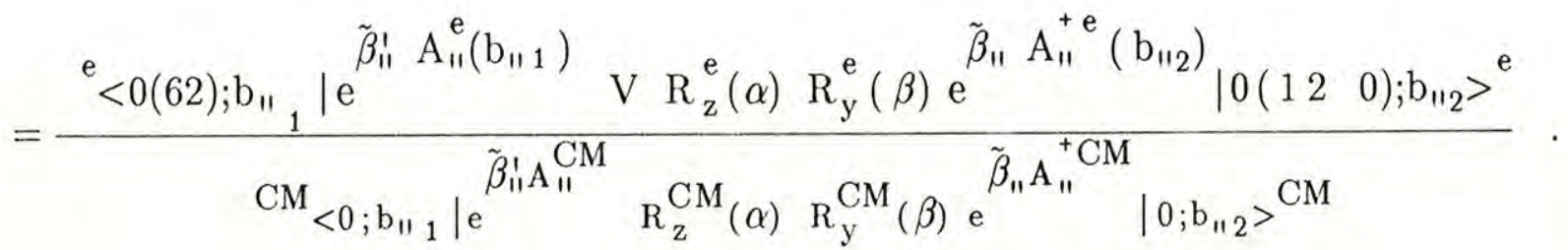


Analogamente à seção anterior, obtemos para o numerador da expressão acima o seguinte:

$$
\begin{aligned}
& { }^{\mathrm{e}}<0(62) ; \mathrm{b}_{11}\left|\mathrm{e}^{\tilde{\beta}_{\| 1}^{\prime} \mathrm{A}_{11}^{\mathrm{e}}\left(\mathrm{b}_{11}\right)} \mathrm{VR}_{\mathrm{z}}^{\mathrm{e}}(\alpha) \mathrm{R}_{\mathrm{y}}^{\mathrm{e}}(\beta) \mathrm{e}^{\tilde{\beta}_{11} \mathrm{~A}_{11}^{+\mathrm{e}}\left(\mathrm{b}_{112}\right)}\right| 0(120) ; \mathrm{b}_{112}>^{\mathrm{e}}= \\
& =\sqrt{6} \sum_{\substack{i j \mathrm{~kb} \\
\mathrm{ab} \\
\mathrm{i}<\mathrm{jb} b}}<\phi_{\mathrm{i} ; \mathrm{a}} \phi_{\mathrm{j} ; \mathrm{b}}\left|\mathrm{V}_{12}\right| \tilde{\phi}_{\mathrm{k} ; \mathrm{a}} \tilde{\phi}_{\mathrm{l} ; \mathrm{b}}>\mathrm{C}_{\mathrm{ia} j \mathrm{jb} \mathrm{ka} \mathrm{lb}}^{\{1122\}}
\end{aligned}
$$

$\{1122\}$

onde $\mathrm{C}_{\mathrm{ia}} \mathrm{jb}$ ka lb é o duplo cofator obtido a partir da matriz de overlap abaixo:

$$
\mathrm{B}^{\{1122\}}=\left[\begin{array}{cccc}
\mathrm{B}_{12}^{\mathrm{n}_{+}} & & & \\
& & & 0 \\
& \mathrm{~B}_{12}^{\mathrm{n}-} & & \\
& & \mathrm{B}_{22}^{\mathrm{p}+} & \\
& & & \mathrm{B}_{22}^{\mathrm{p}-}
\end{array}\right]
$$

onde

$$
\mathrm{B}_{12}=\left[\begin{array}{ccc}
<000 \mid 000> & 0 & <000 \mid 2 \tilde{0} 0> \\
0 & <100 \mid 100> & 0 \\
0 & <010 \mid 100> & 0
\end{array}\right]
$$

e

$$
\mathrm{B}_{22}=\left[\begin{array}{ccc}
<000 \mid 0 \tilde{0} 0> & 0 & <000 \mid 2 \tilde{0} 0> \\
0 & <100 \mid 1 \tilde{0} 0> & 0 \\
<200 \mid 0 \tilde{0} 0> & 0 & <200 \mid 2 \tilde{0} 0>
\end{array}\right]
$$


onde

$$
<n_{1}^{\prime} n_{2}^{\prime} n_{3}^{\prime}\left|n_{1} \tilde{n}_{2} n_{3}>=<\phi_{n_{1}^{\prime} n_{2}^{\prime} n_{3}^{\prime}}\left(b_{11}\right)\right| e^{\tilde{\beta}_{\| 1}^{\prime} A_{11}^{e}\left(b_{11}\right)} R_{z}^{e}(\alpha) R_{y}^{e}(\beta) e^{\tilde{\beta}_{11} A_{11}^{+e}\left(b_{112}\right)} \mid \phi_{n_{1} n_{2} n_{3}}\left(b_{112}\right)>.
$$

Usando as técnicas dos apêndices 1 e 2 obtemos que todos os elementos de matriz envolvidos em $\quad \mathrm{B}_{12} \quad$ e $\quad \mathrm{B}_{22}$ acima são diferentes de zero e o cálculo direto resulta que $\operatorname{det} \mathrm{B}_{22} \neq 0$ e $\operatorname{det} \mathrm{B}_{12}=0$. Como det $\mathrm{B}_{22}$ é diferente de zero podemos obter duplo-cofatores a partir da matriz $\mathrm{B}^{\{1122\}}$, eq.(6.12), não nulos. Necessariamente, tais duplo-cofatores vão ter índices envolvendo as linhas e colunas dos blocos $B_{12}$ na eq.(6.11). Podemos mostrar que existem 16 duplo-cofatores não nulos neste caso e portanto existe 0 acoplamento entre as representações $2 p-2 b$ e $4 p-4 b$. Assim a representação $2 p-2 b$ cumpre o papel de acoplar indiretamente as representações $0 \mathrm{p}-0 \mathrm{~b}$ e $4 \mathrm{p}-4 \mathrm{~b}$. No capítulo 3 apresentamos todas as possíveis representações irredutíveis de $\mathrm{SU}(3)$ dentro da camada $\mathrm{p}$ e vimos que existiam apenas duas com $(\mathrm{S}=0 \mathrm{~T}=0)$, isto é, as representações (04) e (20). Portanto a outra representação que seria uma candidata natural a servir de intermediária no acoplamento entre as representações $0 \mathrm{p}-0 \mathrm{~b}$ e $4 \mathrm{p}-4 \mathrm{~h}$ seria a representação de $\operatorname{Sp}(1, \mathrm{R})$ obitda a partir do estado de peso mínimo (20). No entanto como mostramos no capítulo 3, as representações (04), (62) e (12 0) possuem simetria permutacional orbital do tipo [444] e a representação (20) do tipo [4422]. Como o potencial $\mathrm{V}$ comuta com os geradores do grupo de permutações, concluímos imediatamente que (20) não se acopla a nenhuma das representações (04), (62) e (12 0). 


\subsection{Análise dos Resultados Obtidos}

Nesta seção vamos discutir os resultados que obtivemos considerando o acoplamento entre os estados de base dos modelos $\mathrm{Sp}(1, \mathrm{R})$ associados aos estados de peso mínimo $0 p-0 b, 2 p-2 b$ e $4 p-4 b$, que analisamos no capítulo 4 . Vamos utilizar bases com o mesmo número de estados e comprimentos do oscilador obtidos no caṕ́tulo 4. Os nossos resultados são apresentados no lado direito da figura 6.1 .

Do ponto de vista dos níveis de energia de excitação, nossos resultados comparam-se favoravelmente ao experimento, em relação aos resultados obtidos por Bouten et al ${ }^{(15)}$, em um cálculo do tipo PAV para os estados $0^{+}$, incluindo as representações (04), (62) e (12 0) de SU(3) desacopladas, e também ao cálculo PAV de Abgrall et al ${ }^{(10)}$ que considera as representações (04) e (12 0) de SU(3). A força usada por Bouten também é a B1 de Brink-Boecker, Abgrall usa outra força que também pode ser parametrizada na forma dada no capítulo 4.

Notamos na figura 6.1, que a transição monopolar entre os estados $0_{2}^{+}$e $0_{1}^{+}$e a quadrupolar entre os estados $0_{2}^{+}$e $2_{1}^{+}$é bastante subestimada pelo modelo. Atribuímos este comportamento ao fato do acoplamento obtido através da representação $2 \mathrm{p}-2 \mathrm{~b}$ ser muito fraco.

O mecanismo responsável pelas transições eletromagnéticas dentro do modelo $\mathrm{Sp}(1, \mathrm{R})$ com acoplamento, pode ser melhor compreendido se fizermos uma análise em uma base deformada. Vamos analisar de maneira qualitativa o elemento de matriz de transição monopolar $\mathrm{M}\left(0_{2}^{+} \rightarrow 0_{1}^{+}\right)$em uma base deformada envolvendo apenas a projeção dos estados do vácuo do fonon deformado das representações $0 p-0 b, 2 p-2 b$ e $4 p-4 b$. Representaremos os estados do vácuo, que analisamos no capítulo 5 , com $\mathrm{L}=0$ por $|0 p-0 b>| 2 p-,2 b>$ e $\mid 4 p-4 b>$.

Os dois primeiros estados $0^{+}$, são dados na base acima pela seguinte expressão: 


$$
\left|0_{\nu}^{+}\right\rangle=\mathrm{C}_{0}^{\nu}|0 \mathrm{p}-0 \mathrm{~b}\rangle+\mathrm{C}_{2}^{\nu}|2 \mathrm{p}-2 \mathrm{~b}\rangle+\mathrm{C}_{4}^{\nu}|4 \mathrm{p}-4 \mathrm{~b}\rangle \quad ; \quad \nu=1,2
$$

e o elemento de matriz de transição monopolar elétrica pela expressão abaixo:

$$
\begin{aligned}
<0_{1}\left|\mathrm{M}_{\mathrm{el}}\right| 0_{2}^{+}> & =\mathrm{C}_{0}^{1} \mathrm{C}_{0}^{2}<0 \mathrm{p}-0 \mathrm{~b}\left|\mathrm{M}_{\mathrm{el}}\right| 0 \mathrm{p}-0 \mathrm{~b}>+\mathrm{C}_{2}^{1} \mathrm{C}_{2}^{2}<2 \mathrm{p}-2 \mathrm{~b}\left|\mathrm{M}_{\mathrm{el}}\right| 2 \mathrm{p}-2 \mathrm{~b}>+ \\
& +\mathrm{C}_{4}^{1} \mathrm{C}_{4}^{2}<4 \mathrm{p}-4 \mathrm{~b}\left|\mathrm{M}_{\mathrm{el}}\right| 4 \mathrm{p}-4 \mathrm{~b}>
\end{aligned}
$$

$\mathrm{Na}$ tabela 6.1 mostramos os valores das amplitudes $\mathrm{C}_{\mathrm{n}}^{\nu}$, eq.(6.13) obtidas pela diagonalização da hamiltoniana para os primeiros dois estados $0^{+}$e na tabela 6.2 os elementos de matriz de monopolo elétrico nos estados do vácuo do fonon deformado das representações $0 \mathrm{p}-0 \mathrm{~b}, 2 \mathrm{p}-2 \mathrm{~b}$ e $4 \mathrm{p}-4 \mathrm{~b}$ projetados em $\mathrm{L}=0$.

\begin{tabular}{llll}
\hline & $\mathrm{C}_{0}^{\nu}$ & $\mathrm{C}_{2}^{\nu}$ & $\mathrm{C}_{4}^{\nu}$ \\
\hline$\nu=1$ & 0.999 & 0.037 & 0.004 \\
$\nu=2$ & 0.007 & -0.078 & -0.997 \\
\hline
\end{tabular}

Tab. 6.1. Valores das amplitudes $\mathrm{C}_{\mathrm{n}}^{\nu}, \nu=1,2, \mathrm{n}=0,2,4$, eq.(6.13) dos primeiros estados $0^{+}$, obtidas pela diagonalização da hamiltoniana B1 na base deformada consistindo apenas na projeção em $\mathrm{L}=0$, dos estados do vácuo do fonon deformado das representações $0 p-0 b, 2 p-2 b$ e $4 p-4 b$. Os comprimentos do oscilador deformado são os mesmos que foram obtidos no capítulo 5 . 


\begin{tabular}{ccc}
\hline$\left.<0 \mathrm{p}-0 \mathrm{~b}\left|\mathrm{M}_{\mathrm{el}}\right| 0 \mathrm{p}-0 \mathrm{~b}\right\rangle$ & $<2 \mathrm{p}-2 \mathrm{~b}\left|\mathrm{M}_{\mathrm{el}}\right| 2 \mathrm{p}-2 \mathrm{~b}>$ & $<4 \mathrm{p}-4 \mathrm{~b}\left|\mathrm{M}_{\mathrm{el}}\right| 4 \mathrm{p}-4 \mathrm{~b}>$ \\
\hline 40 & 50 & 69 \\
\hline
\end{tabular}

Tab. 6.2. Elementos de matriz de monopolo elétrico na base deformada consistindo na projeção em $\mathrm{L}=0$ do vácuo do fonon deformado das representações $0 \mathrm{p}-0 \mathrm{~b}$, $2 p-2 b$ e $4 p-4 b$. Os comprimentos do oscilador deformado foram obtidos no capítulo 5 .

Na tabela 6.1 vemos que devido ao acoplamento ser muito fraco os estados $0_{1}^{+}$e $0_{2}^{+}$são totalmente dominados respectivamente pelas representações $0 \mathrm{p}-0 \mathrm{~b}$ e $4 \mathrm{p}-4 \mathrm{~b}$, assim a mistura de representações é muito pequena.

$\mathrm{O}$ elemento de matriz de transição monopolar elétrica $\mathrm{M}$, eq.(6.14), consiste em três termos onde cada um é dado pelo produto das amplitudes dos estados $0_{1}^{+}$e $0_{2}^{+}$pelo elemento de matriz do monopolo elétrico correspondente. A partir da análise das tabelas 6.1 e 6.2 , notamos que o produto das amplitudes envolvidas no cálculo de $\mathrm{M}$ é muito pequeno e mesmo com o elemento de matriz de monopolo sendo da ordem de 50, a transição ainda é muito pequena. Note que há um grande cancelamento entre os termos associados ao estado $|0 \mathrm{p}-0 \mathrm{~b}\rangle$ e $|4 \mathrm{p}-4 \mathrm{~b}\rangle$ na eq.(6.14) devido à ortogonalidade da função de onda.

Para finalizar, convém citar que Takigawa e Arima ${ }^{(21)}$ realizaram um cálculo no modelo de cluster usando o método de Brink, que considera o ${ }^{12} \mathrm{C}$ como sendo descrito por três clusters- $\alpha$, fixos em centros diferentes e onde os quatro nucleons de cada cluster- $\alpha$ ocupam o estado (000) do oscilador harmônico em relação a um sistema de coordenadas fixo no centro do cluster. 
A banda rotacional fundamental é associada a uma configuração espacial onde os três centros dos clusters- $\alpha$ são dispostos nos vértices de um triângulo equilátero e o primeiro estado $0^{+}$excitado é associado a uma configuração dada por uma cadeia linear dos três clusters $-\alpha$.

As funções de onda e níveis de energia são obtidos pela diagonalização de uma hamiltoniana microscópica nos estados dados pela projeção em momento angular das configurações descritas acima. A distância entre os clusters- $\alpha$ e o comprimento do oscilador de cada cluster- $\alpha$ são parâmetros variacionais.

De maneira qualitativa, podemos comparar o cálculo acima com o que realizamos. Abaixo apresentamos o element de matriz de transição monopolar elétrica entre os estados $0_{2}^{+}$e $0_{1}^{+}$e o BE2 entre os estados $0_{2}^{+}$e $2_{1}^{+}$, obtidos por Takigawa e Arima utilizando a interação V2 de Volkov ${ }^{(22)}$ :

$$
\begin{aligned}
& \mathrm{M}\left(0_{2}^{+} \longrightarrow 0_{1}^{+}\right)=0.019 \mathrm{fm}^{2} \\
& \mathrm{~B}\left(\mathrm{E} 2,0_{2}^{+} \longrightarrow 2_{1}^{+}\right)=4.4 \times 10^{-5} \mathrm{e}^{2} \cdot \mathrm{fm}^{4} .
\end{aligned}
$$

Tanto o resultado acima quanto os nossos não reproduzem os dados experimentais, no entanto notamos que os nossos são sensivelmente maiores que os dados acima.

Um cálculo que reproduz bem os dados experimentais, é uma extensão do cálculo de Takigawa e Arima feita por Uegaki et al ${ }^{(23)}$ e que considera um grande número de configuraçòes espaciais envolvendo triângulos equiláteros, isósceles, não-isósceles e cadeiais lineares. Os resultados obtidos por esses autores para o $\mathrm{M}\left(0_{2}^{+} \rightarrow 0_{1}^{+}\right)$e $\mathrm{B}\left(\mathrm{E} 2,0_{2}^{+} \rightarrow 2_{1}^{+}\right)$, usando a interação V1 de Volkov são os seguintes: 


$$
\begin{aligned}
& \mathrm{M}\left(0_{2}^{+} \rightarrow 0_{1}^{+}\right)=6.6 \mathrm{fm}^{2} \\
& \mathrm{~B}\left(\mathrm{E} 2,0_{2}^{+} \longrightarrow 2_{1}^{+}\right)=3.5 \mathrm{e}^{2} \cdot \mathrm{fm}^{4} .
\end{aligned}
$$

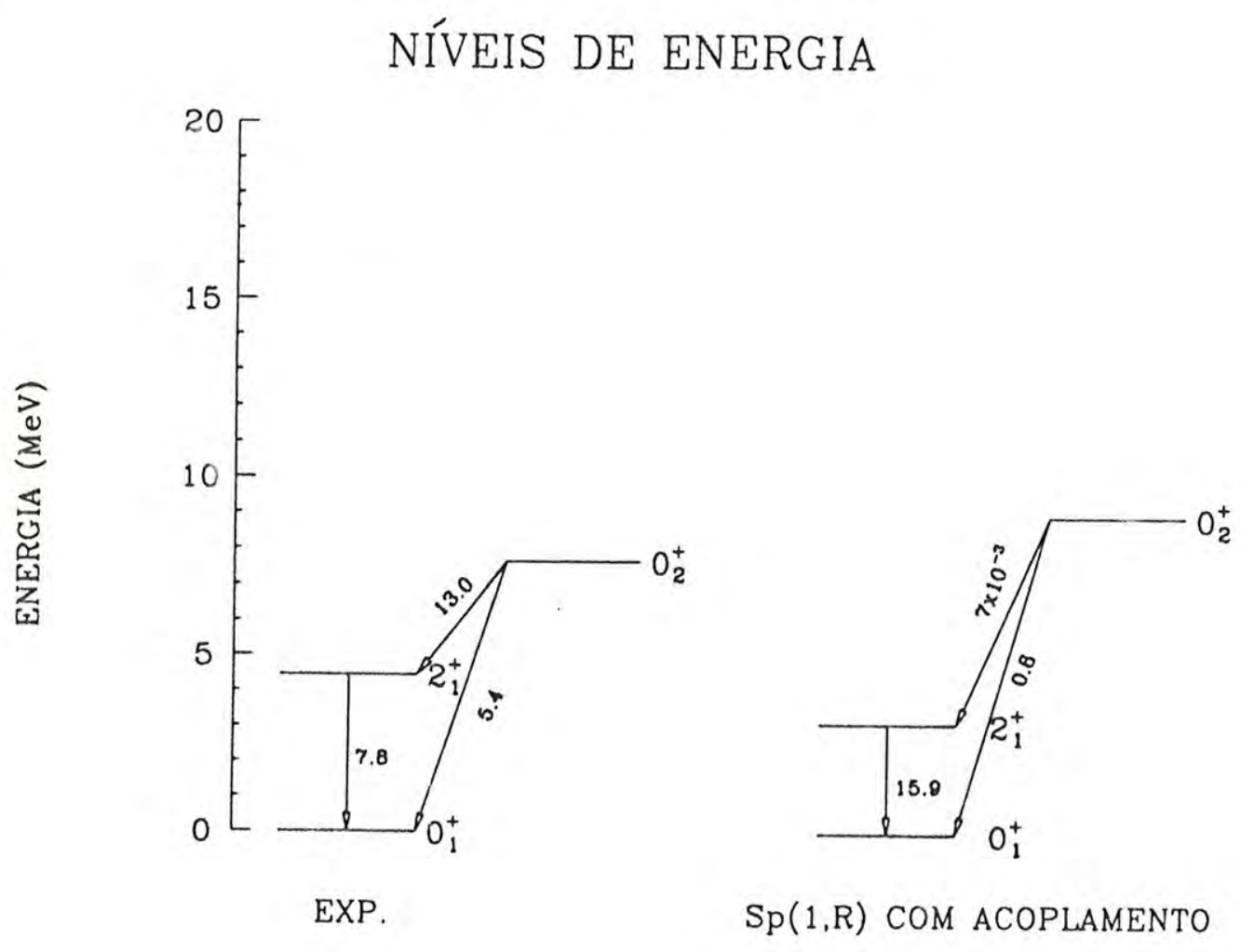

Fig. 6.1. Níveis de energia de excitação, elemento de matriz de transição monopolar elétrica e $\mathrm{B}(\mathrm{E} 2)$ experimentais e calculados pelo modelo $\mathrm{Sp}(1, \mathrm{R})$ considerando a mistura das representações irredutíveis $0 \mathrm{p}-0 \mathrm{~b}, 2 \mathrm{p}-2 \mathrm{~b}$ e $4 \mathrm{p}-4 \mathrm{~b}$. Dados experimentais obtidos na ref.(23) (Energias em MeV, BE2 em $\mathrm{e}^{2} \cdot \mathrm{fm}^{4}$ e $\mathrm{M}_{\mathrm{el}}$ em $\mathrm{fm}^{2}$ ). 


\section{Conclusãr}

$\mathrm{Na}$ breve revisão que fizemos do Modelo Simplético no capítulo 2, mostramos que esse modelo era construído para descrever as vibrações monopolares e quadrupolares e as rotações do núcleo atômico. Vimos que o subespaço coletivo do SCM era determinado pelo seu estado de peso mínimo e era, a priori, um subespaço do espaço de Hilbert do modelo de camadas do oscilador harmônico.

Para estudarmos as propriedades da base de estados do SCM, neste trabalho de tese, escolhemos descrever o núcleo ${ }^{12} \mathrm{C}$ através desse modelo. No capítulo 3 discutimos o procedimento que usamos para obter os estados de peso mínimo de $\operatorname{Sp}(3, \mathrm{R})$ que são adequados à descrição deste núcleo. A análise feita neste capítulo, além de ser necessária aos desenvolvimentos dos capítulos posteriores, teve o objetivo de apresentar as idéias dispersas na literatura de forma sintética, sistemática e organizada.

Devido à enorme dificuldade, tanto do ponto de vista algébrico quanto computacional de implementar o esquema de cálculo no SCM, na forma discutida no capítulo 2, apenas cálculos esquemáticos envolvendo o SCM completo, são encontrados na literatura até o presente momento. Assim, é essencial considerarmos submodelos computacionalmente viáveis do SCM que incorporam apenas os graus de liberdade que, em princípio são os mais relevantes para o problema em estudo. É importante salientar que na maioria dos casos esses submodelos descrevem de maneira qualitativa o SCM completo. Um dos submodelos do SCM que é frequentemente utilizado é o modelo $\operatorname{Sp}(1, R)$ de

Arickx et al ${ }^{(3)}$. Portanto procuramos descrever os estados de baixa energia do ${ }^{12} \mathrm{C}$ através deste modelo objetivando estudar de maneira qualitativa a base de estados do SCM.

No capítulo 4 mostramos como são contruídas as bases de estados dos modelos $\mathrm{Sp}(1, \mathrm{R})$, obtidas a partir de funções de onda de partícula independente do oscilador 
harmônico esférico e como são calculados os elementos de matriz dos observáveis nessas bases. Com o objetivo de estudar com a maior generalidade as bases de $\mathrm{Sp}(1, \mathrm{R})$ consideramos as bases obtidas a partir dos estados de peso mínimo oblato, prolato e triaxial. Nesse mesmo capítulo vimos que os estados da representação oblata consistem em vibrações transversais e geram o modelo $\mathrm{Sp}_{\perp}(1, \mathrm{R})$, o qual associamos aos estados da banda rotacional fundamental do ${ }^{12} \mathrm{C}$. Os estados da representação prolata, consistem em vibrações longitudinais e geram o modelo $\mathrm{Sp}_{11}(1, \mathrm{R})$ que associamos ao estado excitado $\mathrm{O}_{2}^{+}$, observado a uma energia de excitação de $7.65 \mathrm{MeV}$, e finalmente os estados triaxiais geram um outro submodelo $\operatorname{Sp}_{11}(1, \mathrm{R})$ que em princípio podia ser associado ao estado excitado $0_{3}^{+}$com energia de excitação de $10.3 \mathrm{MeV}$.

A análise dos resultados obtidos no capítulo 4 mostrou que para descrevermos os operadores de interesse através do modelo $\mathrm{Sp}(1, \mathrm{R})$ era necessário considerar bases com um grande número de estados. Frequentemente são encontradas bases onde até 20 estados têm contribuição não desprezível. Portanto, ficou evidente a importância das configurações excitadas do modelo $\mathrm{Sp}(1, \mathrm{R})$. Em cálculos $\mathrm{Sp}(1, \mathrm{R})$ encontrados na literatura ${ }^{(7,8)}$ para outros núcleos também é observado o mesmo comportamento. Isto obscurece o entendimento dos mecanismos responsáveis pelo aparecimento das configurações excitadas bem como introduz problemas de convergência nos cálculos, podendo até inviabilizar o cálculo sistemático envolvendo a mistura de representações irredutíveis de $\operatorname{Sp}(3, \mathrm{R})$.

No nosso ponto de vista, para resolvermos o problema acima, é essencial notarmos que em núcleos leves as correlações advindas da deformação são de grande importância sugerindo o uso de uma base otimizada, isto é, uma base construída a partir de funções de onda de partícula independente do oscilador harmônico deformado. No contexto do SCM, como os operadores geradores de transformação de escala pertencem à álgebra $s p(3, R)$, os cálculos nas bases esférica e deformada são completamente equivalentes. 
No capítulo 5 fizemos uma análise usando uma base otimizada dos modelos $\mathrm{Sp}(1, \mathrm{R})$ com estados de peso mínimo oblato, prolato e triaxial que foram discutidos no capítulo 4 em uma base esférica. Isto consiste em uma extensão do trabalho que fizemos na ref.(9) para o núcleo ${ }^{8} \mathrm{Be}$. Mostramos para os três modelos $\mathrm{Sp}(1, \mathrm{R})$ descritos acima, que bases deformadas com no máximo cinco estados eram praticamente equivalentes ao cálculo exato. Vimos inclusive que, para os estados da banda rotacional fundamental, na maioria dos casos, bases que consistiam apenas na projeção do estado do vácuo do fonon deformado, eram uma excelente aproximação do cálculo exato. Mostramos que excessões à regra acima ocorrem em bases triaxiais quando há um grande cancelamento dos termos dominantes. Isto ocorre porque nesse caso existem estados quase degenerados com o mesmo momento angular.

$\mathrm{O}$ momento de quadrupolo elétrico $\mathrm{Q}_{\mathrm{el}}\left(2_{1}^{+}\right)$da representação triaxial $2 \mathrm{p}-2 \mathrm{~b}$ é um exemplo desta excessão, onde vimos que a base obtida pela projeção do estado do vácuo com $\mathrm{K}=0$ e $\mathrm{K}=2$, contribuia com menos do que $50 \%$ do valor exato. Assim vemos que 0 modelo $\mathrm{Sp}(1, \mathrm{R})$ pode ser considerado como uma generalização do modelo PAV do oscilador harmônico deformado de Bouten \& Bouten ${ }^{(11)}$ e Abgrall et al ${ }^{(10)}$. Nesse modelo considera-se apenas os estados obtidos pela projeção do vácuo do fonon deformado, que é um estado de peso $\left[\begin{array}{l}\text { máximo } \\ \text { mínimo }\end{array}\right]$ de $\mathrm{SU}(3)$ deformado. No nosso trabalho vimos que para representações com $\lambda, \mu \neq 0$ isto não é suficiente, sendo necessário considerarmos a projeção de estados com fonons da ordem de $\mathrm{N}=5$.

Como consequência da análise descrita acima, concluímos que o cálculo na base otimizada é factível e resolve os problemas de interpretação física bem como da convergência do cálculo na base esférica.

No contexto do SCM, como os operadores momento de monopolo e quadrupolo pertecem à álgebra $s p(3, R)$, transições monopolares e quadrupolares entre representações irredutíveis distintas de $\mathrm{Sp}(3, \mathrm{R})$ são proibidas. No capítulo 6 vimos, por exemplo, que os 
estados $0_{1}^{+}$e $2_{1}^{+}$do ${ }^{12} \mathrm{C}$ são associados à representação oblata de $\mathrm{Sp}_{\perp}(1, \mathrm{R})$ e o estado intruso $0_{2}^{+}$à representação prolata de $\operatorname{Sp}_{\| \prime}(1, R)$ e portanto as transições monopolares entre os estados $0_{2}^{+}$e $0_{1}^{+}$e quadrupolares entre os estados $0_{2}^{+}$e $2_{1}^{+}$são proibidas por construção. No entanto, essas transições são observadas experimentalmente e, portanto, somos naturalmente levados a considerar o acoplamento entre as representações oblata e prolata. Nesse mesmo capítulo mostramos que uma interação de dois corpos do tipo considerado no capítulo 4, envolvendo apenas os termos de Wigner e Majorana não acoplava diretamente as representações acima.

Portanto, é necessário considerar uma terceira representação irredutível para, de modo indireto obtermos o acoplamento procurado. Através de um cálculo exploratório mostramos que a representação triaxial $2 \mathrm{p}-2 \mathrm{~b}$, cumpria o papel de acoplar indiretamente as representações oblata ou $0 \mathrm{p}-0 \mathrm{~b}$ e prolata ou $4 \mathrm{p}-4 \mathrm{~b}$. A transição monopolar entre os estados $0_{2}^{+}$e $0_{1}^{+}$e a quadrupolar entre os estados $0_{2}^{+}$e $2_{1}^{+}$observadas experimentalmente são bastante subestimadas pelo modelo $\mathrm{Sp}(1, \mathrm{R})$ envolvendo o acoplamento. A partir da análise feita neste capítulo concluímos que isto se deve ao fato do acoplamento ser muito fraco, sugerindo que talvez seja necessário incluir outras representações intermediárias afim de aumentar a intensidade da transição. No contexto do modelo $\operatorname{Sp}(1, \mathrm{R})$, esta é a primeira tentativa de descrever as propriedades do estado intruso $0_{2}^{+}$a partir da representação $4 p-4 b$ e do seu acoplamento com a representação $0 p-0 b$ através da representação $2 p-2 b$.

Nos apêndices 1 e 2 descrevemos as técnicas algébricas que desenvolvemos para realizar os cálculos nos modelos $\mathrm{Sp}(1, \mathrm{R})$, bem como o acoplamento entre eles. Nossas técnicas têm a vantagem de serem facilmente generalizadas para incluir outros graus de liberdade do SCM, além de ser muito mais simples do que as usadas por outros autores, que utilizam procedimentos que envolvem derivadas de determinantes, tornando bastante difícil a análise do acoplamento entre representações irredutíveis de $\operatorname{Sp}(1, \mathrm{R})$. 
Apêndice 1 - Cálculo Detalhado dos Elementos de Matriz dos Operadores de Interesse

O objetivo deste apêndice é mostrar com detalhes suficientes, como realizamos os cálculos dos elementos de matriz dos operadores de interesse nos submodelos $\operatorname{Sp}(1, \mathrm{R})$, associados aos estados de peso mínimo $0 \mathrm{p}-0 \mathrm{~b}, 2 \mathrm{p}-2 \mathrm{~b}$ e $4 \mathrm{p}-4 \mathrm{~b}$ que descrevemos no capítulo 4. Analisaremos apenas o cálculo do overlap e da energia potencial que são feitos através do MFG e apresentam as maiores dificuldades. O cálculo dos elementos de matriz de operadores que é feito por procedimentos algébricos não é difícil e portanto vamos omití-lo.

\section{A.1.1 - Cálculo Através do MFG}

Como mostramos no capítulo 4, expressão (4.32), os elementos de ma riz de um operador $\hat{O}$, escalar na base de estados do modelo $\mathrm{Sp}(1, \mathrm{R}), \mid \mathrm{n}, \mathrm{LMK} ; \mathrm{b}_{0}>$ são obtidos pelo MFG por:

$$
\begin{aligned}
& <\mathrm{n}^{\prime}, \mathrm{L}^{\prime} \mathrm{M}^{\prime} \mathrm{K}^{\prime} ; \mathrm{b}_{0}|\hat{\mathrm{O}}| \mathrm{n}, \mathrm{LMK} ; \mathrm{b}_{0}>=\frac{\Gamma(2 \mathrm{k}) \delta_{\mathrm{LL}^{\prime}} \delta_{\mathrm{MM}^{\prime}}}{\left(\Gamma\left(\mathrm{n}^{\prime}+1\right) \Gamma(\mathrm{n}+1) \Gamma\left(\mathrm{n}^{\prime}+2 \mathrm{k}\right) \Gamma(\mathrm{n}+2 \mathrm{k})\right)^{1 / 2}} \cdot \\
& \cdot \frac{\partial^{\mathrm{n}^{\prime}}}{\partial \tilde{\beta}^{\prime \mathrm{n}^{\prime}}} \frac{\partial^{\mathrm{n}}}{\partial \tilde{\beta}^{\mathrm{n}}}<0 ; \mathrm{b}_{0}\left|\mathrm{e}^{\tilde{\beta}^{\prime} \mathrm{A}} \hat{\mathrm{O}} \mathrm{P}_{\mathrm{K}^{\prime} \mathrm{L}}^{\mathrm{L}} \mathrm{e}^{\tilde{\beta} \mathrm{A}^{+}}\right| 0 ; \mathrm{b}_{0}>\left.\right|_{\begin{array}{l}
\tilde{\beta}^{\prime}=0 \\
\tilde{\beta}=0
\end{array}}
\end{aligned}
$$

onde a função geratriz:

$$
<0 ; \mathrm{b}_{0}\left|\mathrm{e}^{\tilde{\beta}^{\prime} \mathrm{A}} \hat{\mathrm{O}} \mathrm{P}_{\mathrm{K}^{\prime} \mathrm{K}}^{\mathrm{L}} \mathrm{e}^{\tilde{\beta} \mathrm{A}^{+}}\right| 0 ; \mathrm{b}_{0}>
$$


é dada no caso não axialmente simétrico pela expressão (4.33) e no caso axialmente simétrico por (4.34).

O nosso objetivo neste apêndice é obter a expressão (2) e depois substitú́-la na expressão (1), para obtermos os elementos de matriz do operador Ô através das derivadas indicadas.

\section{A.1.2 - Cálculo do Overlap}

No cálculo dos elementos de matriz do overlap, utilizaremos sempre o procedimento que descrevemos na seção 4.4 .2 do capítulo 4. Nesse capítulo mostramos que para obtermos os elementos de matriz do overlap através do MFG, precisamos primeiro calcular a expressão (4.37) que em seguida substituímos em (4.33), para enfim obtermos os elementos de matriz desejados através de (4.32).

\section{A.1.2.1 - Cálculo do Overlap na Representação 0p-0b}

Na seção 4.3.1 do capítulo 4, mostramos que neste caso a base de estados do modelo $\mathrm{Sp}_{\perp}(1, \mathrm{R})$ é dada pelos estados axialmente simétricos $\left|\mathrm{n}(04), \mathrm{LM} 0 ; \mathrm{b}_{0}\right\rangle$. No caso presente, o numerador da expressão (4.37) é dado por:

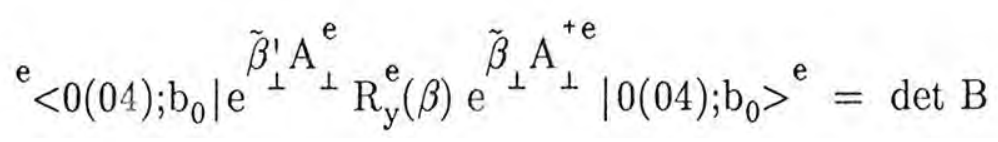

onde (B) ${ }_{\alpha \beta}=\left\langle\phi_{\alpha} \mid \tilde{\phi}_{\beta}\right\rangle$, na notação da seção 4.4.2. A partir da análise feita na seção 4.4.2 e da expressão (4.9), segue que as funções de onda de partícula independente adequadas a este caso são dadas por: 


$$
\begin{aligned}
& \left|\phi_{\alpha}\right\rangle=\mathrm{e}^{\tilde{\beta}_{\perp}^{\prime} \mathrm{A}_{\perp}^{+\mathrm{e}}} \phi_{\mathrm{i}}(\overrightarrow{\mathrm{x}}) \xi_{\mathrm{a}} \\
& \left|\tilde{\phi}_{\beta}\right\rangle=\mathrm{R}_{\mathrm{y}}^{\mathrm{e}(\beta)} \mathrm{e}^{\tilde{\beta}_{\perp} \mathrm{A}_{\perp}^{+\mathrm{e}} \phi_{\mathrm{j}}(\overrightarrow{\mathrm{x}}) \xi_{\mathrm{b}}}
\end{aligned}
$$

onde os rótulos $i, j$ relativos à função de onda orbital indicam a ocupação das funções de onda do oscilador $(000),(010),(001)$ e os rótulos a,b indicam a ocupação dos quatro estados de spin-isospin. Portanto obtemos:

$$
{ }^{(\mathrm{B})}{ }_{\alpha \beta}=<\phi_{\alpha}\left|\tilde{\phi}_{\beta}>=<\phi_{\mathrm{i}}\right| \mathrm{e}^{\tilde{\beta}_{\perp}^{\prime} \mathrm{A}_{\perp}^{\mathrm{e}}} \mathrm{R}_{\mathrm{y}}^{\mathrm{e}}(\beta) \mathrm{e}^{\tilde{\beta}_{\perp} \mathrm{A}_{\perp}^{+\mathrm{e}}} \mid \phi_{\mathrm{j}}>\delta_{\mathrm{ab}}=\mathrm{B}_{\mathrm{ij}} \delta_{\mathrm{ab}}
$$

onde $\mathrm{B}_{\mathrm{ij}}$ é uma matriz $3 \times 3$ e lembramos que usamos a mesma notação para os operadores de $\operatorname{sp}(3, R)$ que agem em um ou em A-nucleons, a distinção fica clara do contexto. Por conveniência, vamos introduzir a seguinte notação:

$$
<n_{1}^{\prime} n_{2}^{\prime} n_{3}^{\prime}\left|n_{1} \tilde{n}_{2} n_{3}>=<\phi_{n_{1}^{\prime} n_{2}^{\prime} n_{3}^{\prime}}\right| e^{\tilde{\beta}^{\prime} A^{e}} R^{e}(\Omega) e^{\tilde{\beta} A^{+e}} \mid \phi_{n_{1} n_{2} n_{3}}>
$$

onde de acordo com o caso que estivermos considerando identificamos os operadores envolvidos na expressão acima. No caso presente temos:

$$
<n_{1}^{\prime} n_{2}^{\prime} n_{3}^{\prime}\left|n_{1} \tilde{n}_{2} n_{3}>=<\phi_{n_{1}^{\prime} n_{2}^{\prime} n_{3}^{\prime}}\right| e^{\tilde{\beta}_{\perp}^{\prime} A_{\perp}^{e}} R_{y}^{e}(\beta) e^{\tilde{\beta}_{\perp} A_{\perp}^{+e}} \mid \phi_{n_{1} n_{2} n_{3}}>
$$

Portanto usando (4) em (3) obtemos: 


$$
{ }^{\mathrm{e}}<0(04) ; \mathrm{b}_{0}\left|\mathrm{e}^{\tilde{\beta}_{\perp}^{\prime} \mathrm{A}_{\perp}^{\mathrm{e}}} \mathrm{R}_{\mathrm{y}}^{\mathrm{e}}(\beta) \mathrm{e}^{\tilde{\beta}_{\perp} \mathrm{A}_{\perp}^{+\mathrm{e}}}\right| 0(04) ; \mathrm{b}_{0}>^{\mathrm{e}}=\operatorname{det} \mathrm{B}=\left(\operatorname{det} \mathrm{B}_{11}\right)^{4}
$$

onde

$$
\begin{aligned}
\mathrm{B}_{11} & =\left[\begin{array}{ccc}
<000 \mid 0 \tilde{0} 0> & <000 \mid 0 \tilde{1} 0> & <000 \mid 0 \tilde{1} 1> \\
<010 \mid 0 \tilde{0} 0> & <010 \mid 0 \tilde{1} 0> & <010 \mid 0 \tilde{1} 1> \\
<001 \mid 0 \tilde{0} 0> & <001 \mid 0 \tilde{1} 0> & <001 \mid 0 \tilde{1} 1>
\end{array}\right]= \\
& =\left[\begin{array}{ccc}
<000 \mid 0 \tilde{0} 0> & 0 & 0 \\
0 & <010 \mid 0 \tilde{1} 0> & 0 \\
0 & 0 & <001 \mid 0 \tilde{1} 1>
\end{array}\right] .
\end{aligned}
$$

Devido à paridade os elementos de matriz não diagonais de $\mathrm{B}_{11}$ se anulam. Portanto para obtermos a expressão (3), basta calcularmos os elementos de matriz de $\mathrm{B}_{11}$ dados acima. A técnica que utilizaremos no cálculo dos elementos de matriz da forma (6), é sugerida notando-se que o operador que aparece entre os estados do oscilador, isto é:

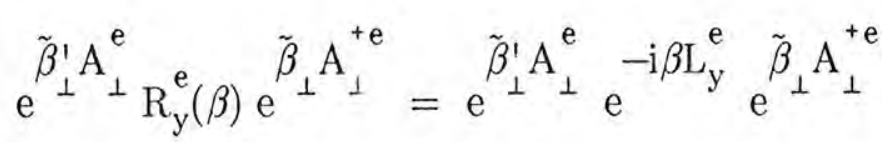

pode ser escrito em função da álgebra $\mathrm{sp}(3, \mathrm{R})$. Portanto podemos escrever o operador acima em sua forma normal onde definimos a forma normal de um operador como sendo a sua representação onde a direita deixamos os operadores abaixadores da álgebra $\operatorname{sp}(3, \mathrm{R})$, a esquerda os levantadores e entre estes os operadores de peso da álgebra.

No apêndice 2 mostramos como obtemos a forma normal de todos os operadores que vamos necessitar. Em particular na seção A.2.3.1, fazendo-se $\mu \longrightarrow \infty$ obtemos: 


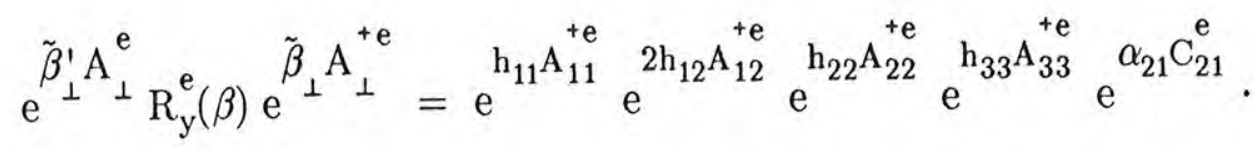

$$
\begin{aligned}
& \cdot \mathrm{e}^{\alpha_{11} \mathrm{C}_{11}^{\mathrm{e}}} \mathrm{e}^{\alpha_{22} \mathrm{C}_{22}^{\mathrm{e}}} \mathrm{e}^{\alpha_{33} \mathrm{C}_{33}^{\mathrm{e}}} . \\
& \cdot \mathrm{e}^{\alpha_{12} \mathrm{C}_{12}^{\mathrm{e}}} \mathrm{e}^{\mathrm{h}_{11}^{\prime} \stackrel{\mathrm{e}}{\mathrm{e}}} \mathrm{e}^{2 \mathrm{~h}_{12}^{\prime} \mathrm{A}_{12}^{\mathrm{e}}} \mathrm{e}^{\mathrm{h}_{22}^{\prime} \mathrm{A}_{22}^{\mathrm{e}}} \mathrm{e}^{\mathrm{h}_{33}^{\prime} \mathrm{A}_{33}^{\mathrm{e}}}
\end{aligned}
$$

onde

$$
\begin{aligned}
& \mathrm{h}_{11}=\frac{\operatorname{sen}^{2} \beta \tilde{\beta}_{\perp}}{\mathrm{P}} \mathrm{h}_{11}^{\prime}=\frac{\operatorname{sen}^{2} \beta \tilde{\beta}_{\perp}^{\prime}}{\mathrm{P}} \alpha_{11}=\ln \left[\cos \beta \frac{\mathrm{G}}{\mathrm{P}}\right] \\
& \mathrm{h}_{12}=(-) \frac{\cos \beta \operatorname{sen} \beta \tilde{\beta}_{\perp}}{\mathrm{P}} \mathrm{h}_{12}^{\prime}=\frac{\cos \beta \operatorname{sen} \beta \tilde{\beta}_{\perp}^{\prime}}{\mathrm{P}} \alpha_{22}=\ln \left[\frac{1}{\cos \beta} \mathrm{G}\right] \\
& \mathrm{h}_{22}=\frac{\cos ^{2} \beta \tilde{\beta}_{\perp}}{\mathrm{P}} \mathrm{h}_{22}^{\prime}=\frac{\cos ^{2} \beta \tilde{\beta}_{\perp}^{\prime}}{\mathrm{P}} \alpha_{33}=\ln \left[\frac{1}{\mathrm{G}}\right] \\
& \mathrm{h}_{33}=\frac{\tilde{\beta}_{\perp}}{\mathrm{G}} \mathrm{h}_{33}^{\prime}=\frac{\tilde{\beta}_{\perp}^{\prime}}{\mathrm{G}} \alpha_{12}=-\alpha_{21}=-\frac{\sin \beta}{\cos \beta} \frac{1}{\mathrm{G}}
\end{aligned}
$$

e

$$
\begin{aligned}
& \mathrm{P}=1-\tilde{\beta}_{\perp} \tilde{\beta}_{\perp}^{\prime} \cos ^{2} \beta \\
& \mathrm{G}=1-\tilde{\beta}_{\perp} \tilde{\beta}_{\perp}^{\prime} .
\end{aligned}
$$

A partir da expressão (8) é trivial o cálculo dos elementos de matriz de $B_{11}$, por exemplo: 


$$
\begin{aligned}
<000|000\rangle & =<000\left|\mathrm{e}^{\alpha_{11} \mathrm{C}_{11}^{\mathrm{e}}} \mathrm{e}^{\alpha_{22} \mathrm{C}_{22}^{\mathrm{e}}} \mathrm{e}^{\alpha_{33} \mathrm{C}_{33}^{\mathrm{e}}}\right| 000>=\mathrm{e}^{1 / 2 \alpha_{11}} \mathrm{e}^{1 / 2 \alpha_{22}} \mathrm{e}^{1 / 2 \alpha_{33}}= \\
& =\frac{1}{\mathrm{G}^{1 / 2}} \frac{1}{\mathrm{P}^{1 / 2}}
\end{aligned}
$$

analogamente

$$
\begin{aligned}
& <010 \mid 0 \tilde{10}>=\frac{\mathrm{x}}{\mathrm{G}^{1 / 2} \mathrm{P}^{3 / 2}} \\
& <001 \mid 0 \tilde{0} 1>=\frac{\mathrm{x}}{\mathrm{G}^{3 / 2} \mathrm{P}^{1 / 2}}
\end{aligned}
$$

onde definimos $\mathrm{x}=\cos \beta$.

Como discutimos no apêndice 2 , a expressão (7) é de caráter puramente algébrico e portanto é imediato que:

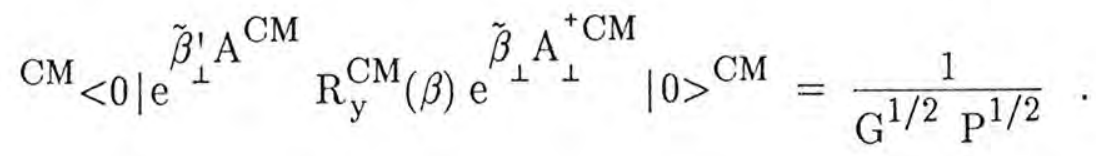

Com os elementos de matriz $B_{11}$ calculados acima, obtemos a expressão (7) que junto com a expressão (9) nos permite o cáculo da expressão (4.37), que mostramos a seguir:

$$
<0(04) ; \mathrm{b}_{0}\left|\mathrm{e}^{\tilde{\beta}_{\perp}^{\prime} \mathrm{A}_{\perp}} \mathrm{R}_{\mathrm{y}}(\beta) \mathrm{e}^{\tilde{\beta}_{\perp} \mathrm{A}_{\perp}^{+}}\right| 0(04) ; \mathrm{b}_{0}>=\frac{\mathrm{x}^{4}}{\mathrm{G}^{19 / 2} \mathrm{P}^{19 / 2}}
$$

Por fim substituimos a expressão acima em (4.34) e (4.32) obtendo os elementos de matriz do overlap: 
$<n^{\prime}(04) L^{\prime} M^{\prime} 0 ; b_{0} \mid n(04), L M 0 ; b_{0}>=\frac{\Gamma(19) \delta_{L_{L}^{\prime}} \delta_{M M}}{\left(\Gamma\left(n^{\prime}+1\right) \Gamma(n+1) \Gamma\left(n^{\prime}+19\right) \Gamma(n+19)\right)^{1 / 2}}$.

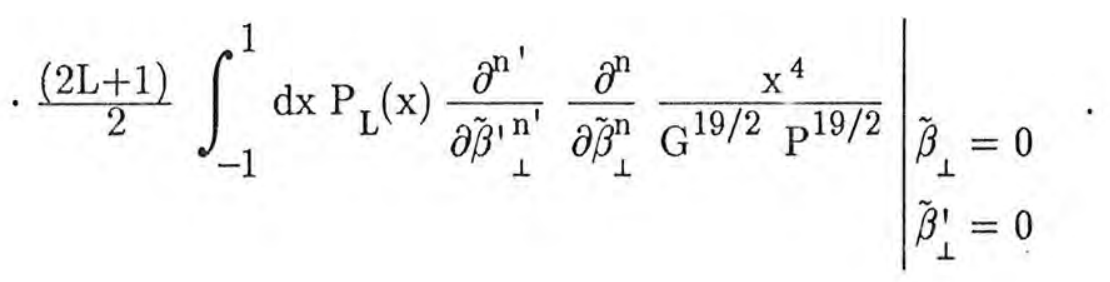

As derivadas envolvidas na expressão acima, podem ser calculadas explicitamente através da regra de Leibnitz para a derivação de um produto de funções:

$$
\frac{\partial^{n}}{\partial y^{n}} f(y) g(y)=\sum_{p=0}^{n}\left(\begin{array}{l}
n \\
p
\end{array}\right) \frac{\partial^{n-p}}{\partial y^{n-p}} f(y) \frac{\partial^{p}}{\partial y^{p}} g(y)
$$

Usando a regra acima, a expressão seguinte

$$
\frac{\partial^{\mathrm{p}}}{\partial \tilde{\beta}_{\perp}^{\mathrm{p}}} \frac{1}{\mathrm{P}^{\mathrm{k}}}=\frac{\Gamma(\mathrm{k}+\mathrm{p})}{\Gamma(\mathrm{k})} \frac{\mathrm{x}^{2 \mathrm{p}}\left(\tilde{\beta}_{\perp}^{\prime}\right)^{\mathrm{p}}}{\mathrm{P}^{\mathrm{k}+\mathrm{p}}}
$$

e notando que $\mathrm{G}=\left.\mathrm{P}(\mathrm{x})\right|_{\mathrm{x}=1}$, obtemos para as derivadas indicadas em (11):

$$
\left.\frac{\partial^{n^{\prime}}}{\partial \tilde{\beta}_{\perp}^{\prime n^{\prime}}} \frac{\partial^{\mathrm{n}}}{\partial \tilde{\beta}_{\perp}^{\mathrm{n}}} \frac{1}{\mathrm{G}^{19 / 2} \mathrm{P}^{19 / 2}}\right|_{\begin{array}{l}
\tilde{\beta}_{\perp}=0 \\
\tilde{\beta}_{\perp}^{\prime}=0
\end{array}}=\frac{\Gamma(\mathrm{n}+1)^{2}}{\Gamma(19 / 2)^{2}} \sum_{\mathrm{p}=0}^{\mathrm{n}} \frac{\Gamma(19 / 2+\mathrm{n}-\mathrm{p}) \Gamma(19 / 2+\mathrm{p}) \mathrm{x}^{2 \mathrm{p}}}{\Gamma(\mathrm{n}-\mathrm{p}+1) \Gamma(\mathrm{p}+1)} \delta_{n n^{\prime}}
$$

Definindo 


$$
A_{L}^{\mu+2 n}=\frac{(2 L+1)}{2} \int_{-1}^{1} d x P_{L}(x) x^{\mu+2 n}
$$

e substituindo (12) em (11) obtemos finalmente os elementos de matriz do overlap:

$$
\begin{aligned}
& <\mathrm{n}^{\prime}(04), \mathrm{L}^{\prime} \mathrm{M}^{\prime} 0 ; \mathrm{b}_{0} \mid \mathrm{n}(04), \mathrm{LM} 0 ; \mathrm{b}_{0}>=\delta_{\mathrm{LL}^{\prime}} \delta_{\mathrm{MM}^{\prime}} \delta_{\mathrm{nn}^{\prime}} \cdot \\
& \cdot \frac{\Gamma(19) \Gamma(\mathrm{n}+1)}{\Gamma(19 / 2)^{2} \Gamma(\mathrm{n}+19)} \sum_{\mathrm{p}=0}^{\mathrm{n}} \frac{\Gamma(19 / 2+\mathrm{n}-\mathrm{p}) \Gamma(19 / 2+\mathrm{p})}{\Gamma(\mathrm{n}-\mathrm{p}+1) \Gamma(\mathrm{p}+1)} \mathrm{A}_{\mathrm{L}}^{4+2 \mathrm{p}} .
\end{aligned}
$$

$\mathrm{Na}$ expressão acima notamos que a base de estados de $\operatorname{Sp}_{\perp}(1, R)$ é ortogonal e os valores do momento angular orbital $\mathrm{L}$ são pares e restritos a $\mathrm{L} \leq 4+2 \mathrm{n}$. A expressão acima tem uma forma adequada ao cálculo numérico no computador.

\section{A.1.2.2 - Cálculo do Overlap na Representação $2 \mathrm{p}-2 \mathrm{~b}$}

No capítulo 4, seção 4.3.2, mostramos que a base de estados do modelo $\operatorname{Sp}_{11}(1, \mathrm{R})$ associarlo ao estado de peso mínimo $2 \mathrm{p}-2 \mathrm{~b}$ é dada pelos estados não axialmente simétricos $\mid \mathrm{n}(62), \mathrm{LMK} ; \mathrm{b}_{0}>$. O estado de peso mínimo dado pela expressão (4.13), consiste em uma soma de seis determinantes de Slater e portanto não podemos aplicar diretamente o procedimento utilizado na seção anterior, que era adequado ao overlap entre dois determinantes de Slater. No entanto, como veremos a seguir, a generalização deste último procedimento é óbvia.

A partir da expressão (4.13), obtemos para o numerador da expressão (4.37): 


$$
\begin{aligned}
& { }^{\mathrm{e}}<0(62) ; \mathrm{b}_{0}\left|\mathrm{e}^{\tilde{\beta}_{\| 1}^{\prime} \mathrm{A}_{11}^{\mathrm{e}}} \mathrm{R}^{\mathrm{e}}(\Omega) \mathrm{e}^{\tilde{\beta}_{11} \mathrm{~A}_{\| 1}^{+\mathrm{e}}}\right| 0(62) ; \mathrm{b}_{0}>{ }^{\mathrm{e}}= \\
& =\frac{1}{6} \sum_{\left\{\mathrm{C}^{\prime}\right\},\{\mathrm{C}\}}<\psi^{\left\{\mathrm{C}^{\prime}\right\}} ; \mathrm{b}_{0}\left|\mathrm{e}^{\tilde{\beta}_{\|}^{\prime} \mathrm{A}_{\|}^{\mathrm{e}}} \mathrm{R}^{\mathrm{e}}(\Omega) \mathrm{e}^{\tilde{\beta}_{\|} \mathrm{A}_{\|}^{+\mathrm{e}}}\right| \psi^{\{\mathrm{C}\}} ; \mathrm{b}_{0}>
\end{aligned}
$$

onde a notação usada é a mesma da seção 4.3.2.

A expressão acima consiste em uma soma de 36 termos onde cada termo é dado pelo produto escalar de dois estados determinantais. Portanto, cada um destes termos pode ser calculado pelo procedimento que discutimos na seção 4.4 .2 e que foi utilizado na seção anterior. Vamos mostrar que esses 36 termos podem ser classificados em três grupos de termos semelhantes onde todos os termos de um mesmo grupo dão contribuição idêntica ao overlap e portanto, em princípio só temos que calcular três produtos escalares entre estados determinantais.

Analisando a estrutura do estado (4.13), notamos que a cada um dos quatro estados de spin-isospin estão associados três estados espaciais e portanto é fácil ver que todos os 36 termos da expressão (13), que correspondem ao overlap entre dois estados determinantais são da forma:

$$
\operatorname{det} \mathrm{B}=\operatorname{det}\left[\begin{array}{ccccc}
\mathrm{Bl}_{1}^{\mathrm{n}_{+}} & & & \\
& & & 0 \\
& \mathrm{Bl}_{2}^{\mathrm{n}-} & & \\
& & \mathrm{Bl}_{3}^{\mathrm{p}} & \\
& & & \mathrm{Bl}_{4}^{\mathrm{p}-}
\end{array}\right]
$$


onde os blocos $\mathrm{Bl}_{1}, \mathrm{Bl}_{2}, \mathrm{Bl}_{3}, \mathrm{Bl}_{4}$ correspondem as matrizes $3 \times 3$ relacionadas ao overlap dos estados orbitais, os índices superiores $\mathrm{n}+, \mathrm{n}-, \mathrm{p}+, \mathrm{p}-$, indicam os estados de spin-isospin associados às funções de onda orbitais de cada bloco e $\mathrm{B}$ é uma matriz $12 \times 12$. Por exemplo:

$$
\begin{aligned}
& { }^{\mathrm{e}}<\psi^{\{1122\}} ; \mathrm{b}_{0}\left|\mathrm{e}^{\tilde{\beta}_{\| 1}^{\prime} \mathrm{A}_{\| 1}^{\mathrm{e}}} \mathrm{R}(\Omega)^{\mathrm{e}} \mathrm{e}^{\tilde{\beta}_{\| 1} \mathrm{~A}_{\|}^{+\mathrm{e}}}\right| \psi^{\{1212\}} ; \mathrm{b}_{0}>^{\mathrm{e}}= \\
& =\operatorname{det}\left[\begin{array}{cccc}
\mathrm{B}_{11}^{\mathrm{n}+} & & & \\
& & & 0 \\
& \mathrm{~B}_{12}^{\mathrm{n}-} & & \\
& & \mathrm{B}_{21}^{\mathrm{p}+} & \\
& & & \\
& & & \mathrm{B}_{22}^{\mathrm{p}-}
\end{array}\right]
\end{aligned}
$$

onde as matrizes acima são definidas por:

$$
\begin{aligned}
& \mathrm{B}_{11}=\left[\begin{array}{ccc}
<000 \mid 0 \tilde{0} 0> & 0 & 0 \\
0 & <100 \mid 1 \tilde{0} 0> & <100 \mid 0 \tilde{1} 0> \\
0 & <010 \mid 1 \tilde{0} 0> & <010 \mid 0 \tilde{1} 0>
\end{array}\right] \\
& \mathrm{B}_{12}=\left[\begin{array}{ccc}
<000 \mid 0 \tilde{0} 0> & 0 & <000 \mid 2 \tilde{0} 0> \\
0 & <100 \mid 100> & 0 \\
0 & <010 \mid 100> & 0
\end{array}\right] \\
& \mathrm{B}_{21}=\left[\begin{array}{ccc}
<000 \mid 0 \tilde{0} 0> & 0 & 0 \\
0 & <100 \mid 100> & <100 \mid 0 \tilde{1} 0> \\
<200 \mid 0 \tilde{0} 0> & 0 & 0
\end{array}\right]
\end{aligned}
$$




$$
\mathrm{B}_{22}=\left[\begin{array}{ccc}
<000 \mid 0 \tilde{0} 0> & 0 & <000 \mid 2 \tilde{0} 0> \\
0 & <100 \mid 10 \tilde{0} 0> & 0 \\
<200 \mid 0 \tilde{0} 0> & 0 & <200 \mid 2 \tilde{0} 0>
\end{array}\right]
$$

a notação usada é definida pela expressão (5), na situação adequada a esta seção.

Observando a estrutura dos 36 termos (13) podemos classificá-los em 3 grupos, onde em cada grupo as matrizes $\mathrm{Bl}_{\mathrm{i}}, \mathrm{i}=1,4$, eq.(14) são dados por permutações de um mesmo conjunto de matrizes como mostramos a seguir:

\section{Grupo 1}

6 termos que são obtidos quando os blocos $\mathrm{Bl}_{\mathrm{i}}, \mathrm{i}=1,4$ em (14) são dados pelas seis permutações distintas das matrizes $\mathrm{B}_{11}, \mathrm{~B}_{11}, \mathrm{~B}_{22}, \mathrm{~B}_{22}$

\section{Grupo 2}

6 termos que são obtidos quando os blocos $\mathrm{Bl}_{\mathrm{i}}, \mathrm{i}=1,4$ em (14) são dados pelas seis permutações distintas das matrizes $\mathrm{B}_{12}, \mathrm{~B}_{12}, \mathrm{~B}_{21}, \mathrm{~B}_{21}$

\section{Grupo 3}

24 termos que são obtidos quando os blocos $\mathrm{Bl}_{\mathrm{i}}, \mathrm{i}=1,4$ em (14) são dados pelas 24 permutações distintas das matrizes $\mathrm{B}_{11}, \mathrm{~B}_{22}, \mathrm{~B}_{12}, \mathrm{~B}_{21}$.

Com o que discutimos acima já temos condições de calcular a expressão (13). É fácil ver que todos os termos de um mesmo grupo acima vão dar a mesma contribuição à expressão (13) uma vez que os rótulos de spin-isospin são irrelevantes neste caso, portanto obtemos: 


$$
\begin{aligned}
& { }^{\mathrm{e}}<0(62) ; \mathrm{b}_{0}\left|\mathrm{e}^{\tilde{\beta}_{\| 1}^{\prime} \mathrm{A}_{\| 1}^{\mathrm{e}}} \mathrm{R}(\Omega)^{\mathrm{e}} \mathrm{e}^{\tilde{\beta}_{\| 1} \mathrm{~A}_{\| 1}^{+\mathrm{e}}}\right| 0(62) ; \mathrm{b}_{0}>{ }^{\mathrm{e}}= \\
& =\operatorname{det}\left[\begin{array}{llll}
\mathrm{B}_{11} & & & 0 \\
& \mathrm{~B}_{11} & & \\
& & \mathrm{~B}_{22} & \\
& & & \mathrm{~B}_{22}
\end{array}\right]+\operatorname{det}\left[\begin{array}{cccc}
\mathrm{B}_{12} & & & \\
& & & \\
& \mathrm{~B}_{12} & & \\
& & \mathrm{~B}_{21} & \\
& & & \\
0 & & & \mathrm{~B}_{21}
\end{array}\right]+ \\
& +4 \operatorname{det}\left[\begin{array}{llll}
\mathrm{B}_{11} & & & 0 \\
& \mathrm{~B}_{22} & & \\
& & \mathrm{~B}_{12} & \\
& & & \mathrm{~B}_{21}
\end{array}\right]=\left(\operatorname{det} \mathrm{B}_{11}\right)^{2}\left(\operatorname{det} \mathrm{B}_{22}\right)^{2}
\end{aligned}
$$

onde na expressão acima usamos que $\operatorname{det} \mathrm{B}_{12}=\operatorname{det} \mathrm{B}_{21}=0$, o que é imediato da definição de tais matrizes dadas na eq.(15).

Para calcularmos a expressão (16) precisamos calcular o $\operatorname{det} B_{11}$ e $\operatorname{det} B_{22}$. Usando a técnica de obter a forma normal do operador $\mathrm{e}^{\tilde{\beta}_{\| 1}^{\mathrm{l}} \mathrm{A}_{\| 1}^{\mathrm{e}}} \mathrm{R}(\Omega)^{\mathrm{e}} \mathrm{e}^{\tilde{\beta}_{\| 1} \mathrm{~A}_{\|}^{+\mathrm{e}}}$ como descrevemos no apêndice 2 obtemos:

$$
\begin{aligned}
& <000\left|00 \tilde{0}>=\frac{1}{\mathrm{P}^{1 / 2}} ;<100\right| 0 \tilde{1} 0>=(-) \frac{\operatorname{sen} \beta \cos \gamma}{\mathrm{P}^{3 / 2}} \\
& <100\left|10 \tilde{0} 0>=\frac{\mathrm{x}}{\mathrm{P}^{3 / 2}} ;<010\right| 10 \tilde{0} 0>=\frac{\operatorname{sen} \beta \cos \alpha}{\mathrm{P}^{3 / 2}}
\end{aligned}
$$




$$
\begin{aligned}
& <010 \mid 0 \tilde{1} 0>=\frac{1}{\mathrm{x}}\left[\frac{\bar{\Omega}}{\mathrm{P}^{1 / 2}}-\frac{\cos \alpha \cos \gamma \operatorname{sen}^{2} \beta}{\mathrm{P}^{3 / 2}}\right] \\
& <000\left|200>=\frac{1}{\sqrt{2}} \frac{\mathrm{x}^{2} \tilde{\beta}_{1 \prime}^{\prime}}{\mathrm{P}^{3 / 2}} ;<200\right| 00 \tilde{0} 0>\frac{1}{\sqrt{2}} \frac{\mathrm{x}^{2} \tilde{\beta}_{11}}{\mathrm{P}^{3 / 2}} \\
& <200 \mid 200>=\mathrm{x}^{2}\left[-\frac{1}{2} \frac{1}{\mathrm{P}^{3 / 2}}+\frac{3}{2} \frac{1}{\mathrm{P}^{5 / 2}}\right]
\end{aligned}
$$

onde definimos $\mathrm{P}=1-\mathrm{x}^{2} \tilde{\beta}_{11} \tilde{\beta}_{\| 1}^{\prime}$ e $\bar{\Omega}=\cos \alpha \cos \gamma-\operatorname{sen} \alpha \operatorname{sen} \gamma \cos \beta$. Portanto obtemos:

$$
\begin{aligned}
\operatorname{det} \mathrm{B}_{11} & =-\frac{\bar{\Omega}}{\mathrm{P}^{5 / 2}} \\
\operatorname{det} \mathrm{B}_{22} & =\frac{\mathrm{x}^{3}}{\mathrm{P}^{9 / 2}} .
\end{aligned}
$$

Substituindo os determinantes calculados acima em (16) e notando que

$$
\mathrm{CM}_{<0 \mid \mathrm{e}} \mathrm{e}^{\tilde{\beta}_{\| 1}^{\prime \prime} \mathrm{A}_{\|}^{\mathrm{CM}}} \mathrm{R}^{\mathrm{CM}}(\Omega) \mathrm{e}^{\tilde{\beta}_{\| 1} \mathrm{~A}_{\|}^{+} \mathrm{CM}} \mid 0>^{\mathrm{CM}}=\frac{1}{\mathrm{P}^{1 / 2}}
$$

obtemos para a expressão (4.37):

$$
<0(62) ; \mathrm{b}_{0}\left|\mathrm{e}^{\tilde{\beta}_{\|}^{\prime} \mathrm{A}_{\|}} \mathrm{R}(\Omega) \mathrm{e}^{\tilde{\beta}_{11} \mathrm{~A}_{\| \prime}^{+}}\right| 0(62) ; \mathrm{b}_{0}>=\frac{\bar{\Omega}^{2} \mathrm{x}^{6}}{\mathrm{P}^{27 / 2}} .
$$

Substituindo a expressão acima em (4.33) e (4.32) obtemos: 


$$
\begin{aligned}
& <\mathrm{n}^{\prime}(62), \mathrm{L}^{\prime} \mathrm{M}^{\prime} \mathrm{K} ; \mathrm{b}_{0} \mid \mathrm{n}(62), \mathrm{LMK} ; \mathrm{b}_{0}>=\frac{\delta_{\mathrm{LL}^{\prime}} \delta_{M M}, \Gamma(27 / 2)}{\left(\Gamma\left(\mathrm{n}^{\prime}+1\right) \Gamma(\mathrm{n}+1) \Gamma\left(\mathrm{n}^{\prime}+27 / 2\right) \Gamma(\mathrm{n}+27 / 2)\right)^{1 / 2}} \cdot \\
& \left.\frac{(2 \mathrm{~L}+1)}{8 \pi^{2}} \int \mathrm{d} \Omega \mathrm{D}_{\mathrm{K}^{\prime} \mathrm{K}}(\Omega)^{*} \mathrm{x}^{6} \bar{\Omega}^{2} \frac{\partial^{\mathrm{n}^{\prime}}}{\partial \tilde{\beta}_{\| \prime}^{\prime n^{\prime}}} \frac{\partial^{\mathrm{n}}}{\partial \tilde{\beta}_{\| \prime}^{\mathrm{n}}} \frac{1}{\mathrm{P}^{27 / 2}}\right|_{\begin{array}{l}
\tilde{\beta}_{\prime \prime}=0 \\
\tilde{\beta}_{\prime \prime}^{\prime}=0
\end{array}} \cdot
\end{aligned}
$$

Realizando as derivadas indicadas acima e usando a expressão (4.25) obtemos:

$$
\begin{aligned}
& <n^{\prime}(62) L^{\prime} M^{\prime} K^{\prime} ; b_{0} \mid n(62), L M K ; b_{0}>=\delta_{L L^{\prime}} \delta_{M M^{\prime}} \delta_{n n^{\prime}} \cdot \\
& \frac{(2 L+1)}{8 \pi^{2}} \int_{0}^{2 \pi} \mathrm{d} \alpha \int_{-1}^{1} \mathrm{dx} \int_{0}^{2 \pi} \mathrm{d} \gamma \mathrm{e}^{\mathrm{i} \alpha \mathrm{K}^{\prime}} \mathrm{d}_{\mathrm{K}^{\prime} \mathrm{K}^{\mathrm{L}}}^{\mathrm{L}}(\mathrm{x}) \mathrm{e}^{\mathrm{i} \gamma \mathrm{K}} \bar{\Omega}^{2} \mathrm{x}^{6+2 \mathrm{n}} .
\end{aligned}
$$

A expressão acima é adequada para o cálculo numérico. Como iremos considerar valores de L baixos, o nosso procedimento consiste em substituir a forma explícita da matriz de rotação reduzida e realizar as integrais analiticamente.

\section{A.1.2.3 - Cálculo do Overlap da Representação 4p-4b}

Como este caso é inteiramente análogo ao caso $0 \mathrm{p}-0 \mathrm{~b}$, vamos ser mais concisos. A partir da análise feita na seção 4.4.2, e da expressão (4.18) obtemos:

$$
{ }^{\mathrm{e}}<0(120) ; \mathrm{b}_{0}\left|\mathrm{e}^{\tilde{\beta}_{\|}^{\prime} \mathrm{A}_{\| \prime}^{\mathrm{e}}} \mathrm{R}_{\mathrm{y}}^{\mathrm{e}}(\beta) \mathrm{e}^{\tilde{\beta}_{\| 1} \mathrm{~A}_{\|}^{+\mathrm{e}}}\right| 0(120) ; \mathrm{b}_{0}>^{\mathrm{e}}=\left(\operatorname{det} \mathrm{B}_{22}\right)^{4}
$$


onde

$$
\mathrm{B}_{22}=\left[\begin{array}{ccc}
<000 \mid 0 \tilde{0} 0> & 0 & <000 \mid 2 \tilde{0} 0> \\
0 & <100 \mid 100> & 0 \\
<200 \mid 000> & 0 & <200 \mid 2 \tilde{0} 0>
\end{array}\right] \text {. }
$$

O determinante da matriz acima, foi calculado na seção anterior.

Substituindo na expressão (4.37) a expressão acima e calculando o denominador de (4.37) obtemos:

$$
<0(120) ; \mathrm{b}_{0}\left|\mathrm{e}^{\tilde{\beta}_{\|}^{\prime} \mathrm{A}_{\| \prime}} \mathrm{R}_{\mathrm{y}}(\beta) \mathrm{e}^{\tilde{\beta}_{\| 1} \mathrm{~A}_{\| \prime}^{+}}\right| 0(120) ; \mathrm{b}_{0}>=\frac{\mathrm{x}^{12}}{\mathrm{P}^{35 / 2}} .
$$

Substituindo a expressão acima em (4.34) e (4.32) e calculando as derivadas indicadas obtemos:

$$
<n^{\prime}(120), L^{\prime} M^{\prime} 0^{\prime} ; b_{0} \mid n(120), L M 0 ; b_{0}>=A_{L}^{12+2 n} \delta_{n n^{\prime}} \delta_{L^{\prime}} \delta_{M M^{\prime}}
$$

onde $A_{L}^{\mu+2 n}$ foi definido na seção A.2.1.

Na expressão acima notamos que a base de estados é ortogonal, os valores de L são pares e satisfazem a restrição $\mathrm{L} \leq 12+2 \mathrm{n}$.

\section{A.1.3 - Cálculo do Potencial}

\section{A.1.3.1 - Cálculo do Potencial através do MFG}

Na seção 4.4 .3 do capítulo 4 discutimos brevemente como poderíamos calcular os elementos de matriz do potencial na base de estados do modelo $\mathrm{Sp}(1, \mathrm{R})$, através do MFG. 
Vimos que o cálculo da função geratriz dada pela expressão (2) se reduzia essencialmente ao cálculo do operador potencial entre um ou uma soma de estados determinantais. O principal objetivo desta seção é mostrar como realizamos tais cálculos.

O operador energia potencial é dado por:

$$
V=\sum_{i<j} V_{i j}
$$

onde a interação de dois corpos $\mathrm{V}_{\mathrm{ij}}$ que iremos efetivamente utilizar é dada pela expressão:

$$
V_{i j}=u\left(r_{i j}\right)\left(V_{0}+V_{M} P_{i j}^{e}\right), \quad u\left(r_{i j}\right)=\exp \left[-\frac{r_{i j}^{2}}{\mu^{2}}\right]
$$

onde $\mathrm{V}_{0}, \mathrm{~V}_{\mathrm{M}}$ correspondem a parâmetros da interação, $\mu$ é o seu alcance, $r_{i j}=|\vec{x}(i)-\vec{x}(j)|$ e $P_{i j}^{e}$ é o operador de troca das coordenadas espaciais dos nucleons " $\mathrm{i}$ " e "j". Notemos que a interação acima não depende do spin e do isospin.

Como vimos no capítulo 4 se $|\phi\rangle$ e $|\tilde{\phi}\rangle$ são dois estados determinantais, o elemento de matriz $\mathrm{V}$ é dado por:

$$
<\phi|\mathrm{V}| \tilde{\phi}\rangle=\frac{1}{2} \sum_{\alpha \beta \gamma \delta}<\phi_{\alpha} \phi_{\beta}\left|\mathrm{V}_{12}\right| \tilde{\phi}_{\gamma} \tilde{\phi}_{\delta}>\mathrm{C}_{\alpha \beta \gamma \delta}
$$

onde os estados de partícula independente acima, referentes ao cálculo no modelo $\mathrm{Sp}(1, \mathrm{R})$ foram discutidos nas seções A.1.2.1-1.2.3 deste apêndice e no capítulo 4 e $\mathrm{C}_{\alpha \beta \gamma \delta}$ é o duplo cofator associado à matriz do overlap $\mathrm{B}$, com elementos de matriz (B) ${ }_{\alpha \beta}=<\phi_{\alpha} \mid \tilde{\phi}_{\beta}>$. 
Uma vez que nos casos associados aos estados de peso mínimo $0 \mathrm{p}-0 \mathrm{~b}$ e $4 \mathrm{p}-4 \mathrm{~b}$ temos $\operatorname{det} B \neq 0$, como vimos nas seções A.1.2.1 e A.1.2.3, vamos analisar esta situação em particular. Se $\operatorname{det} \mathrm{B} \neq 0$ o duplo cofator $\mathrm{C}_{\alpha \beta \gamma \delta}$ pode ser escrito da seguinte maneira:

$$
\mathrm{C}_{\alpha \beta \gamma \delta}=\operatorname{det} \mathrm{B}\left[\left(\mathrm{B}^{-1}\right)_{\gamma \alpha}\left(\mathrm{B}^{-1}\right)_{\delta \beta}-\left(\mathrm{B}^{-1}\right)_{\gamma \beta}\left(\mathrm{B}^{-1}\right)_{\delta \alpha}\right] .
$$

Substituindo a expressão acima em (22) e somando nos rótulos de spin-isospin obtemos:

$$
\langle\phi|\mathrm{V}| \tilde{\phi}\rangle=\langle\phi| \tilde{\phi}>\sum_{\substack{\mathrm{ij} \\ \mathrm{k} 1}}\left[\mathrm{~V}_{\mathrm{D}}<\phi_{\mathrm{i}} \phi_{\mathrm{j}}|\mathrm{u}| \tilde{\phi}_{\mathrm{k}} \tilde{\phi}_{\mathrm{l}}>+\mathrm{V}_{\mathrm{E}}<\phi_{\mathrm{i}} \phi_{\mathrm{j}}|\mathrm{u}| \tilde{\phi}_{1} \tilde{\phi}_{\mathrm{k}}>\right]\left(\mathrm{B}^{-1}\right)_{\mathrm{ki}}\left(\mathrm{B}^{-1}\right)_{\mathrm{lj}}
$$

onde $\mathrm{V}_{\mathrm{D}}=8 \mathrm{~V}_{0}-2 \mathrm{~V}_{\mathrm{M}}$ e $\mathrm{V}_{\mathrm{E}}=8 \mathrm{~V}_{\mathrm{M}}-2 \mathrm{~V}_{0}$ e recordamos que $\mathrm{B}^{-1}$ acima é uma matriz $3 \times 3$. A soma que fizemos nos rótulos de spin-isospin é obtida facilmente notando que $V_{12}$ não depende do spin-isospin e os estados determinantais envolvidos têm simetria permutacional orbital [444]. A expressão acima será utilizada no cálculo do numerador da expressão (4.39) e através das expressões (4.32) e (4.34) obteremos os elementos de matriz $\mathrm{V}$ nos casos $0 \mathrm{p}-0 \mathrm{~b}$ e $4 \mathrm{p}-4 \mathrm{~b}$.

\section{A.1.3.2 - Cálculo do Elemento de Matriz $<\phi_{\mathrm{i}} \phi_{\mathrm{j}}|\mathrm{u}| \tilde{\phi}_{\mathrm{k}} \tilde{\phi}_{1}>$}

Na seção A.1.2 discutimos o cálculo da matriz do overlap B, em todos os casos de interesse e como vimos não apresentava maiores dificuldades. Consequentemente também não será difícil o cálculo de $\mathrm{C}_{\alpha \beta \gamma \delta}$ e $\left(\mathrm{B}^{-1}\right)_{\mathrm{ij}}$ envolvidos nas expressões (22) e (24) acima. 
A principal dificuldade no cálculo das expressões (22) e (24) consiste em obtermos o elemento de matriz $<\phi_{\mathrm{i}} \phi_{\mathrm{j}}|\mathrm{u}| \tilde{\phi}_{\mathrm{k}} \tilde{\phi}_{1}>$, que em uma notação adequada a todos os casos de interesse é dado por:

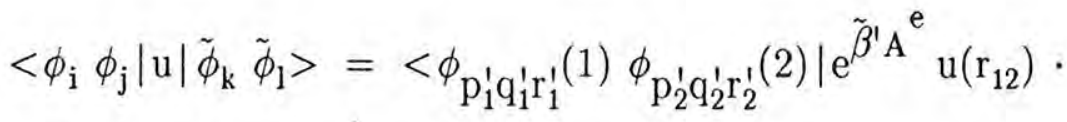

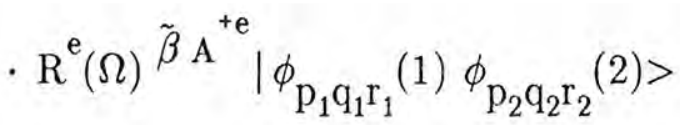

onde $\phi_{\text {pqri }}($ i) corresponde à função de onda do oscilador harmônico na base cartesiana com números quânticos $\mathrm{p}, \mathrm{q}, \mathrm{r}, \mathrm{R}^{\mathrm{e}}(\Omega)$ envolve apenas a rotação nas coordenadas das partículas 1 e 2 e $A^{+e}=A^{+e}(1)+A^{+e}(2), A^{e}=\left[\mathrm{A}^{+e}\right]^{+}$.

Os operadores $\mathrm{A}^{+\mathrm{e}}(\mathrm{i})$ e $\mathrm{A}^{\mathrm{e}}(\mathrm{i})$ são geradores da álgebra $\mathrm{sp}(1, \mathrm{R})$, relativa ao nucleon "i", onde lembramos que identificamos $\mathrm{sp}(1, \mathrm{R})$ ou com a álgebra $\mathrm{sp}_{\|}(1, \mathrm{R})$ ou com $\mathrm{sp}_{\perp}(1, \mathrm{R})$ conforme o caso considerado.

Para calcularmos o elemento de matriz (25) vamos utilizar o procedimento de transformar as coordenadas do par de nucleons em coordenadas relativas e de centro de massa do par de nucleons. A trasformação de coordenadas é obtida definindo as coordenadas e momentos de Jacobi da seguinte maneira ${ }^{(24)}$ :

$$
\begin{aligned}
& {\left[\begin{array}{l}
\vec{\rho} \\
\vec{R}
\end{array}\right]=\left[\begin{array}{cc}
1 / \sqrt{2} & -1 / \sqrt{2} \\
1 / \sqrt{2} & 1 / \sqrt{2}
\end{array}\right]\left[\begin{array}{l}
\overrightarrow{\mathrm{x}}(1) \\
\overrightarrow{\mathrm{x}}(2)
\end{array}\right]} \\
& {\left[\begin{array}{l}
\vec{\pi} \\
\overrightarrow{\mathrm{P}}
\end{array}\right]=\left[\begin{array}{cc}
1 / \sqrt{2} & -1 / \sqrt{2} \\
1 / \sqrt{2} & 1 / \sqrt{2}
\end{array}\right]\left[\begin{array}{l}
\overrightarrow{\mathrm{p}}(1) \\
\overrightarrow{\mathrm{p}}(2)
\end{array}\right]}
\end{aligned}
$$


onde é óbvio que a transformação acima é ortogonal. Se temos

$$
\mathrm{H}^{\mathrm{e}}=\frac{\overrightarrow{\mathrm{p}}(1)^{2}}{2 \mathrm{~m}}+\frac{1}{2} \mathrm{~m} \omega^{2} \overrightarrow{\mathrm{x}}(1)^{2}+\frac{\overrightarrow{\mathrm{p}}(2)^{2}}{2 \mathrm{~m}}+\frac{1}{2} \mathrm{~m} \omega^{2} \overrightarrow{\mathrm{x}}(2)^{2}
$$

por meio da transformação (26) obtemos:

$$
\mathrm{H}^{\mathrm{e}}=\frac{\vec{\pi}^{2}}{2 \mathrm{~m}}+\frac{1}{2} \mathrm{~m} \omega^{2} \vec{\rho}^{2}+\frac{\overrightarrow{\mathrm{P}}^{2}}{2 \mathrm{~m}}+\frac{1}{2} \mathrm{~m} \omega^{2} \overrightarrow{\mathrm{R}}^{2}=\mathrm{H}^{\mathrm{rel}}+\mathrm{H}^{\mathrm{CM}} .
$$

As funções de onda produto $\left.\left|\phi_{p_{1} q_{1} r_{1}}(1) \phi_{p_{2} q_{2} r_{2}}(2)>=\right| p_{1} q_{1} r_{1}, p_{2} q_{2} r_{2}\right\rangle$, são autofunções da hamiltoniana (27). As funções produto $\mid$ pqr $>\mid P Q R>$ são autofunções de (28) onde |pqr> é autofunção da hamiltoniana de oscilador do movimento relativo e |PQR> é autofunção do oscilador do centro de massa do par de nucleons e portanto podemos escrever:

$$
\left|\mathrm{p}_{1} \mathrm{q}_{1} \mathrm{r}_{1}, \mathrm{p}_{2} \mathrm{q}_{2} \mathrm{r}_{2}\right\rangle=\sum_{\substack{\mathrm{pqr} \\ \mathrm{PQR}}}|\mathrm{pqr}, \mathrm{PQR}><\mathrm{pqr}, \mathrm{PQR}| \mathrm{p}_{1} \mathrm{q}_{1} \mathrm{r}_{1}, \mathrm{p}_{2} \mathrm{q}_{2} \mathrm{r}_{2}>.
$$

A transformação acima é conhecida na literatura como transformação de Talmi-Moshinsky. No caso específico das funções de onda do oscilador harmônico em uma base cartesiana, os coeficientes de Talmi-Moshinsky podem ser obtidos por meio de uma expressão fechada, que reproduzimos no apêndice 3 , onde calculamos tais coeficientes nos casos de interesse.

É fácil mostrar que a transformação (26) implica nas seguintes decomposições: 


$$
\begin{aligned}
& \mathrm{A}^{+e}=\mathrm{A}^{+e}(1)+\mathrm{A}^{+e}(2)=\mathrm{A}^{+}+\mathrm{A}^{+} \mathrm{CM} \\
& \mathrm{A}^{\mathrm{e}}=\mathrm{A}^{\mathrm{e}}(1)+\mathrm{A}^{\mathrm{e}}(2)=\mathrm{A}+\mathrm{A}^{\mathrm{CM}} \\
& \overrightarrow{\mathrm{L}}^{\mathrm{e}}=\overrightarrow{\mathrm{L}}^{\mathrm{e}}(1)+\overrightarrow{\mathrm{L}}^{\mathrm{e}}(2)=\overrightarrow{\mathrm{L}}+\overrightarrow{\mathrm{L}}^{\mathrm{CM}}
\end{aligned}
$$

onde $\mathrm{A}^{+}$corresponde ao operador $\mathrm{A}_{\|}^{+}$ou $\mathrm{A}_{\perp}^{+}$que definimos abaixo, $\overrightarrow{\mathrm{L}}^{\mathrm{e}}(\mathrm{i})$ corresponde ao operador momento angular do nucleon "i" em relação a um referencial fixo no espaço, $\overrightarrow{\mathrm{L}}=\vec{\rho} \times \vec{\pi}, \overrightarrow{\mathrm{L}}^{\mathrm{CM}}=\overrightarrow{\mathrm{R}} \times \overrightarrow{\mathrm{P}} \mathrm{e}$

$$
\begin{aligned}
& \mathrm{A}_{11}^{+}=\frac{1}{2} \mathrm{a}_{1}^{+} \mathrm{a}_{1}^{+} \\
& \mathrm{A}_{\perp}^{+}=\frac{1}{2} \mathrm{a}_{2}^{+} \mathrm{a}_{2}^{+}+\frac{1}{2} \mathrm{a}_{3}^{+} \mathrm{a}_{3}^{+} \\
& \mathrm{A}_{11}^{+} \mathrm{CM}=\frac{1}{2} \mathrm{a}_{1}^{+} \mathrm{CM} \mathrm{a}_{1}^{+} \mathrm{CM} \\
& \mathrm{A}_{\perp}^{+} \mathrm{CM}=\frac{1}{2} \mathrm{a}_{2}^{+} \mathrm{CM} \mathrm{a}_{2}^{+} \mathrm{CM}+\frac{1}{2} \mathrm{a}_{3}^{+} \mathrm{CM} \mathrm{a}_{3}^{+} \mathrm{CM}
\end{aligned}
$$

onde

$$
\begin{aligned}
& \mathrm{a}_{\mu}^{+}=\frac{1}{\sqrt{2}}\left[\frac{\rho_{\mu}}{\mathrm{b}_{0}}+\mathrm{i} \frac{\mathrm{b}_{0}}{\hbar} \pi_{\mu}\right], \quad \mu=1,2,3 \\
& \mathrm{a}_{\mu}^{+\mathrm{CM}}=\frac{1}{\sqrt{2}}\left[\frac{\mathrm{R}_{\mu}}{\mathrm{b}_{0}}+\mathrm{i} \frac{\mathrm{b}_{0}}{\hbar} \mathrm{P}_{\mu}\right]
\end{aligned}
$$


onde o índice superior "CM" que se refere a centro de massa é um abuso de notação.

Notando da eq.(26) que $\vec{\rho}=\frac{1}{\sqrt{2}}(\overrightarrow{\mathrm{x}}(1)-\overrightarrow{\mathrm{x}}(2))$, é imediato que $\mathrm{u}\left(\mathrm{r}_{12}\right)=\exp \left(-\mathrm{r}_{12}^{2} / \mu^{2}\right)$, eq.(21), pode ser escrito na forma seguinte:

$$
\mathrm{u}(\rho)=\mathrm{e}^{-2 \rho^{2} / \mu^{2}} .
$$

Usando a trasnformação (29), a decomposição (30) e a expressão acima em (25) obtemos:

$$
\begin{aligned}
& <\mathrm{p}_{1}^{\prime} \mathrm{q}_{1}^{\prime} \mathrm{r}_{1}^{\prime}, \mathrm{p}_{2}^{\prime} \mathrm{q}_{2}^{\prime} \mathrm{r}_{2}^{\prime}\left|\mathrm{e}^{\tilde{\beta}^{\prime} \mathrm{A}} \mathrm{u}\left(\mathrm{r}_{12}\right) \mathrm{R}^{\mathrm{e}}(\Omega)^{\tilde{\beta} \mathrm{A}^{+\mathrm{e}}}\right| \mathrm{p}_{1} \mathrm{q}_{1} \mathrm{r}_{1}, \mathrm{p}_{2} \mathrm{q}_{2} \mathrm{r}_{2}>= \\
& =\sum<\mathrm{p}_{1}^{\prime} \mathrm{q}_{1}^{\prime} \mathrm{r}_{1}^{\prime}, \mathrm{p}_{2}^{\prime} \mathrm{q}_{2}^{\prime} \mathrm{r}_{2}^{\prime}\left|\mathrm{p}^{\prime} \mathrm{q}^{\prime} \mathrm{r}^{\prime}, \mathrm{P}^{\prime} \mathrm{Q}^{\prime} \mathrm{R}^{\prime}><\mathrm{p}_{1} \mathrm{q}_{1} \mathrm{r}_{1}, \mathrm{p}_{2} \mathrm{q}_{2} \mathrm{r}_{2}\right| \mathrm{pqr}, \mathrm{PQR}>. \\
& \cdot<\mathrm{p}^{\prime} \mathrm{q}^{\prime} \mathrm{r}^{\prime}\left|\mathrm{e}^{\tilde{\beta}^{\prime} \mathrm{A}} \mathrm{u}(\rho) \mathrm{R}(\Omega) \tilde{\beta} \mathrm{A}^{+}\right| \mathrm{pqr}><\mathrm{P}^{\prime} \mathrm{Q}^{\prime} \mathrm{R}^{\prime}\left|\mathrm{e}^{\tilde{\beta}^{\prime} \mathrm{A}^{\mathrm{CM}} \mathrm{R}^{\mathrm{CM}}(\Omega)} \tilde{\beta} \mathrm{A}^{+} \mathrm{CM}\right| \mathrm{PQR}>
\end{aligned}
$$

onde deixamos a somatória subentendida.

A seguir usaremos a expressão acima para calcularmos os elementos de matriz do potencial nos casos de interesse.

\section{A.1.3.3 - Cálculo do Potencial na Representação 0p-0b}

O estado $\left|0(04) ; \mathrm{b}_{0}\right\rangle$, dado pela expressão (4.9) tem simetria [444] e como mostramos na seção A.1.3.1 podemos utilizar neste caso a expressão (24) para calcularmos o numerador da expressão (4.39), que reproduzimos abaixo usando a notação adequada a este caso: 


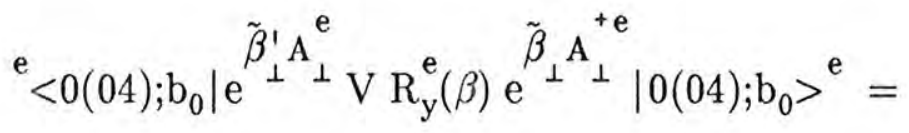

$$
\begin{aligned}
& { }^{\mathrm{e}}<0(04) ; \mathrm{b}_{0}\left|\mathrm{e}^{\tilde{\beta}_{\perp}^{\prime} \mathrm{A}_{\perp}^{\mathrm{e}}} \mathrm{R}_{\mathrm{y}}^{\mathrm{e}}(\beta) \mathrm{e}^{\tilde{\beta}_{\perp} \mathrm{A}_{\perp}^{+\mathrm{e}}}\right| 0(04) ; \mathrm{b}_{0}>^{\mathrm{e}} \\
& \sum_{\mathrm{i} j}\left[\mathrm{~V}_{\mathrm{D}}<\phi_{\mathrm{i}} \phi_{\mathrm{j}}\left|\mathrm{e}^{\tilde{\beta}_{\perp}^{\mathrm{A}} \mathrm{A}_{\perp}^{\mathrm{e}}} \mathrm{u} \mathrm{R}_{\mathrm{y}}^{\mathrm{e}}(\beta) \mathrm{e}^{\tilde{\beta}_{\perp} \mathrm{A}_{\perp}^{+\mathrm{e}}}\right| \phi_{\mathrm{k}} \phi_{1}>+\right. \\
& \left.\left.+\mathrm{V}_{\mathrm{E}}<\phi_{\mathrm{i}} \phi_{\mathrm{j}}\left|\mathrm{e}^{\tilde{\beta}_{\perp}^{\prime} \mathrm{A}_{\perp}^{\mathrm{e}}} \mathrm{u} \mathrm{R}_{\mathrm{y}}^{\mathrm{e}}(\beta) \mathrm{e}^{\tilde{\beta}_{\perp} \mathrm{A}_{\perp}^{+\mathrm{e}}}\right| \tilde{\phi}_{1} \tilde{\phi}_{\mathrm{k}}>\right]\left(\mathrm{B}_{11}^{-1}\right)_{1 \mathrm{j}}\left(\mathrm{B}_{11}^{-1}\right)_{\mathrm{ki}}\right]
\end{aligned}
$$

onde a matriz $B_{11}$ e os estados orbitais $i, j$ foram definidos na seção A.1.2.1.

Por conveniência, vamos definir a notação simplificada:

$$
\begin{aligned}
& <p_{1}^{\prime} q_{1}^{\prime} r_{1}^{\prime} p_{2}^{\prime} q_{2}^{\prime} r_{2}^{\prime}|u| p_{1} \tilde{q}_{1} r_{1} p_{2} \tilde{q}_{2} r_{2}>=
\end{aligned}
$$

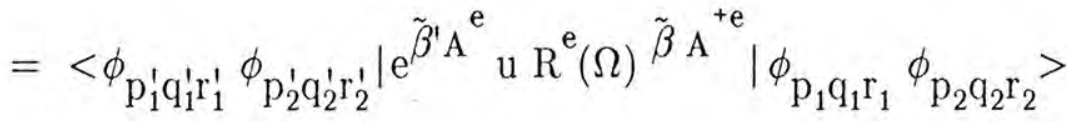

onde interpretamos os operadores acima de acordo com o caso que estivermos considerando.

Na seção A.1.2.1 mostramos que os rótulos i,j,k,l da expressão (32) estão associados aos estados (000),(010) e (001) do oscilador. A partir da expressão (9) e da expressão (32) obtemos para a expressão (4.39) o seguinte: 


$$
\begin{aligned}
& <0(04) ; \mathrm{b}_{0}\left|\mathrm{e}^{\tilde{\beta}_{\perp}^{\prime} \mathrm{A}_{\perp}} \mathrm{u} \mathrm{R}_{\mathrm{y}}(\beta) \mathrm{e}^{\tilde{\beta}_{\perp} \mathrm{A}_{\perp}^{+}}\right| 0(04) ; \mathrm{b}_{0}>= \\
& <0(04) ; \mathrm{b}_{0}\left|\mathrm{e}^{\tilde{\beta}_{\perp}^{\prime} \mathrm{A}_{\perp}} \mathrm{R}_{\mathrm{y}}(\beta) \mathrm{e}^{\tilde{\beta}_{\perp} \mathrm{A}_{\perp}^{+}}\right| 0(04) ; \mathrm{b}_{0}> \\
& \cdot\left\{[ \mathrm { V } _ { \mathrm { D } } + \mathrm { V } _ { \mathrm { E } } ] \left[<000000|\mathrm{u}| 0000000>\left(\mathrm{B}_{11}^{-1}\right)_{000000^{+}}^{2}\right.\right. \\
& \left.+<010010|\mathrm{u}| 0 \tilde{1} 00 \tilde{1} 0>\left(\mathrm{B}_{11}^{-1}\right)_{010}^{2} 010+<001001|\mathrm{u}| 00 \tilde{1} 0 \tilde{1} 1>\left(\mathrm{B}_{11}^{-1}\right)_{001}^{2} 001\right]+ \\
& +2\left[\mathrm{~V}_{\mathrm{D}}<000010|\mathrm{u}| 00 \tilde{0} 00 \tilde{1} 0>+\mathrm{V}_{\mathrm{E}}<000010|\mathrm{u}| 0 \tilde{0} 0000>\right]\left(\mathrm{B}_{11}^{-1}\right)_{000000}\left(\mathrm{~B}_{11}^{-1}\right)_{010010}+ \\
& +2\left[\mathrm{~V}_{\mathrm{D}}<000001|\mathrm{u}| 00 \tilde{0} 00 \tilde{0} 1>+\mathrm{V}_{\mathrm{E}}<000001|\mathrm{u}| 0001000>\right]\left(\mathrm{B}_{11}^{-1}\right)_{000000}\left(\mathrm{~B}_{11}^{-1}\right)_{001001}+ \\
& \left.+2\left[\mathrm{~V}_{\mathrm{D}}<010001|\mathrm{u}| 0 \tilde{1} 00 \tilde{1} 1>+\mathrm{V}_{\mathrm{E}}<010001|\mathrm{u}| 0 \tilde{0} 10 \tilde{1} 0>\right]\left(\mathrm{B}_{11}^{-1}\right)_{001001}\left(\mathrm{~B}_{11}^{-1}\right)_{010} 010\right\}
\end{aligned}
$$

Na seção A.1.2.1, obtivemos a expressão (10) e a matriz $B_{11}$, portanto basta calcularmos os elementos de matriz (33) que ocorrem acima para concluirmos o cálculo da expressão (34).

O cálculo de elementos de matriz da forma (33) e feito utilizando-se o procedimento descrito na seção A.1.3.2, que consiste em separar as funções de onda produto em componentes relativa e de centro de massa do par de nucleons, que resulta na expressão (31). Os coeficientes de Talmi-Moshinsky necessários ao cálculo de (31) foram obtidos no apêndice 3 para todos os casos de interesse. O cálculo dos operadores 


$$
\begin{aligned}
& \mathrm{e}^{\tilde{\beta}_{\perp}^{\prime} \mathrm{A}_{\perp}} \mathrm{u} \mathrm{R}_{\mathrm{y}}(\beta) \mathrm{e}^{\tilde{\beta}_{\perp} \mathrm{A}_{\perp}^{+}} \\
& \mathrm{e}^{\tilde{\beta}_{\perp}^{\prime} \mathrm{A}_{\perp}^{\mathrm{CM}}} \mathrm{R}_{\mathrm{y}}^{\mathrm{CM}}(\beta) \mathrm{e}^{\tilde{\beta}_{\perp} \mathrm{A}_{\perp}^{+} \mathrm{CM}}
\end{aligned}
$$

entre funções de onda do oscilador, que ocorrem na expressão (31) são calculados pelo mesmo método que discutimos na seção A.1.2.1 quando calculamos o overlap, ou seja, através do cálculo da forma normal dos operadores envolvidos. No apêndice 2 discutimos como obter a forma normal de tais operadores. Como exemplo vamos mostrar como calcular o termo $<000000 \mid$ u $\mid 00 \tilde{0} 000>$.

No apêndice 3 mostramos que o único coeficiente de Talmi-Moshinsky não nulo neste caso é o seguinte:

$$
<000,000 \mid 000,000>=1
$$

e portanto da expressão (31) obtemos:

$$
\begin{gathered}
<000000|\mathrm{u}| 00 \tilde{0} 0 \tilde{0} 0>=<000\left|\mathrm{e}^{\tilde{\beta}_{\perp}^{\prime} \mathrm{A}_{\perp}} \mathrm{u} \mathrm{R}_{\mathrm{y}}(\beta) \mathrm{e}^{\tilde{\beta}_{\perp} \mathrm{A}_{\perp}^{+}}\right| 000> \\
\cdot<000\left|\mathrm{e}^{\tilde{\beta}_{\perp}^{\prime} \mathrm{A}_{\perp}^{\mathrm{CM}}} \mathrm{R}_{\mathrm{y}}^{\mathrm{CM}}(\beta) \mathrm{e}^{\tilde{\beta}_{\perp} \mathrm{A}_{\perp}^{+} \mathrm{CM}}\right| 000>.
\end{gathered}
$$

Usando a forma normal dos operadores envolvidos na expressão acima obtemos: 


$$
\begin{aligned}
& <000\left|\mathrm{e}^{\tilde{\beta}_{\perp}^{\prime} \mathrm{A}_{\perp}} \mathrm{u} \mathrm{R}_{\mathrm{y}}(\beta) \mathrm{e}^{\tilde{\beta}_{\perp} \mathrm{A}_{\perp}^{+}}\right| 000>=\frac{1}{\bar{\gamma}^{3 / 2} \mathrm{~W}^{1 / 2} \mathrm{Q}^{1 / 2}} \\
& <000\left|\mathrm{e}^{\tilde{\beta}_{\perp}^{\prime} \mathrm{A}_{\perp}^{\mathrm{CM}}} \mathrm{R}_{\mathrm{y}}^{\mathrm{CM}}(\beta) \mathrm{e}^{\tilde{\beta}_{\perp} \mathrm{A}_{\perp}^{+} \mathrm{CM}}\right| 000>=\frac{1}{\mathrm{G}^{1 / 2} \mathrm{P}^{1 / 2}}
\end{aligned}
$$

onde

$$
\begin{aligned}
& \bar{\gamma}=1+2 \frac{\mathrm{b}_{0}^{2}}{\mu^{2}} \\
& \mathrm{~W}=1+\mathrm{a}_{1}\left[\tilde{\beta}_{\perp}+\tilde{\beta}_{\perp}^{\prime}\right]+\mathrm{c}_{1} \tilde{\beta}_{\perp} \tilde{\beta}_{\perp}^{\prime} \\
& \mathrm{Q}=1+\mathrm{a}_{1}\left[\tilde{\beta}_{\perp}+\tilde{\beta}_{\perp}^{\prime}\right]+\mathrm{b}_{1} \tilde{\beta}_{\perp} \tilde{\beta}_{\perp}^{\prime} \\
& \mathrm{a}_{1}=\frac{\bar{\gamma}-1}{\bar{\gamma}}, \quad \mathrm{b}_{1}=\mathrm{a}_{1}^{2}-\frac{\mathrm{x}^{2}}{\bar{\gamma}^{2}} \\
& \mathrm{c}_{1}=\mathrm{a}_{1}^{2}-\frac{1}{\bar{\gamma}^{2}} \quad, \quad \mathrm{x}=\cos \beta
\end{aligned}
$$

portanto

$$
<000000|\mathrm{u}| 0 \tilde{0} 000 \tilde{0} 0>=\frac{1}{\bar{\gamma}^{3 / 2} \mathrm{G}^{1 / 2} \mathrm{P}^{1 / 2} \mathrm{~W}^{1 / 2} \mathrm{Q}^{1 / 2}} .
$$

Calculando de maneira análoga os outros termos e substituindo na expressão (34) obtemos: 


$$
\begin{aligned}
& <0(04) ; \mathrm{b}_{0}\left|\mathrm{e}^{\tilde{\beta}_{\perp}^{\prime} \mathrm{A}_{\perp}} \mathrm{VR}_{\mathrm{y}}(\beta) \mathrm{e}^{\tilde{\beta} \mathrm{A}_{\perp}^{+}}\right| 0(04) ; \mathrm{b}_{0}>=\frac{\mathrm{x}^{4}}{\bar{\gamma}^{3 / 2}} \cdot \\
& \cdot\left\{\frac{5}{2} \mathrm{X}_{+} \frac{1}{\mathrm{G}^{9} \mathrm{~W}^{1 / 2}} \frac{1}{\mathrm{P}^{8} \mathrm{Q}^{1 / 2}}+\left[\frac{1}{8} \mathrm{X}_{+}\left[\frac{\mathrm{a}_{1}^{2}}{\mathrm{x}^{2}}-\left[1+\frac{1}{\bar{\gamma}^{2}}\right]+\frac{3}{4 \bar{\gamma}} \mathrm{X} \cdot\right]\right]\right. \\
& \cdot \frac{1}{\mathrm{G}^{9} \mathrm{~W}^{1 / 2} \mathrm{P}^{8} \mathrm{Q}^{3 / 2}}+\left[\frac{1}{8} \mathrm{X}_{+}\left(\mathrm{c}_{1}-1\right)+\frac{3}{4 \bar{\gamma}} \mathrm{X}_{-}\right] \frac{1}{\mathrm{G}^{8} \mathrm{~W}^{3 / 2} \mathrm{P}^{9} \mathrm{Q}^{1 / 2}}+ \\
& +\frac{1}{4 \bar{\gamma}^{2}} \mathrm{X}_{+} \frac{1}{\mathrm{G}^{8} \mathrm{~W}^{3 / 2} \mathrm{P}^{9} \mathrm{Q}^{3 / 2}}+\frac{3}{8} \frac{\mathrm{X}_{+}}{\bar{\gamma}^{2}} \frac{1}{\mathrm{G}^{7} \mathrm{~W}^{5 / 2} \mathrm{P}^{9} \mathrm{Q}^{1 / 2}}+ \\
& \left.+\frac{3}{8 \bar{\gamma}^{2}} \mathrm{X}_{+} \frac{1}{\mathrm{G}^{9} \mathrm{~W}^{1 / 2} \mathrm{P}^{7} \mathrm{Q}^{5 / 2}}\right\}
\end{aligned}
$$

onde

$$
\begin{aligned}
& \mathrm{X}_{+}=\mathrm{V}_{\mathrm{D}}+\mathrm{V}_{\mathrm{E}}=6\left(\mathrm{~V}_{0}+\mathrm{V}_{\mathrm{M}}\right) \\
& \mathrm{X}_{-}=\mathrm{V}_{\mathrm{D}}-\mathrm{V}_{\mathrm{E}}=10\left(\mathrm{~V}_{0}-\mathrm{V}_{\mathrm{M}}\right) .
\end{aligned}
$$

Substituindo a expressão acima em (4.34) e depois em (4.32) obtemos os elementos de matriz do potencial. Estes elementos podem ser escritos como uma soma de termos da forma:

$$
\left.\left.\int_{-1}^{1} \mathrm{dx} \mathrm{P}_{L}(\mathrm{x}) \mathrm{x}^{\lambda} \frac{\partial^{\mathrm{n}^{\prime}}}{\partial \tilde{\beta}_{\perp}^{1^{\mathrm{n}^{\prime}}}} \frac{\partial^{\mathrm{n}}}{\partial \tilde{\beta}_{\perp}^{\mathrm{n}}} \frac{1}{\mathrm{G}^{\mathrm{t}} \mathrm{W}^{\mathrm{u}} \mathrm{P}^{\mathrm{r}} \mathrm{Q}^{\mathrm{s}}}\right|_{\tilde{\beta}_{\perp}=0}\right|_{\tilde{\beta}_{\perp}^{\prime}=0}
$$


onde as derivadas envolvidas acima podem ser calculadas analiticamente e o resultado integrado $^{(5)}$.

\section{A.1.3.4 - Cálculo do Potencial na Representação 2p-2b}

Analogamente à seção anterior, para obtermos os elementos de matriz do potencial, vamos calcular o numerador da expressão (4.39) onde neste caso o estado $\left|0(62) ; \mathrm{b}_{0}\right\rangle^{\mathrm{e}}$ consiste em uma soma de seis determinantes de Slater, como discutimos na seção A.1.2.2. Logo é fácil ver que o numerador da expressão (4.39) é dado por uma soma de 36 termos onde cada um é da forma dada na expressão (22). O duplo cofator associado a cada termo é obtido a partir do overlap entre os estados determintais correspondentes. Na seção A.1.2.2 analisamos os 36 termos envolvidos no cálculo do overlap.

Usando a notação adequada a esta seção, obtemos para o numerador da expressão (4.39):

$$
\begin{aligned}
& { }^{\mathrm{e}}<0(62) ; \mathrm{b}_{0}\left|\mathrm{e}^{\tilde{\beta}_{\| \mathrm{A}_{\|}^{\mathrm{e}}}^{\mathrm{e}}} \mathrm{VR}^{\mathrm{e}}(\Omega) \mathrm{e}^{\tilde{\beta}_{\| \prime} \mathrm{A}_{\|}^{+\mathrm{e}}}\right| 0(62) ; \mathrm{b}_{0}>^{\mathrm{e}}= \\
& \frac{1}{6} \sum_{\left\{\mathrm{C}^{\prime}\right\}} \sum_{\left\{\mathrm{C}^{-}\right.}{ }^{\mathrm{e}}<\psi^{\left\{\mathrm{C}^{\prime}\right\}} ; \mathrm{b}_{0}\left|\mathrm{e}^{\tilde{\beta}_{\|}^{\prime} \mathrm{A}_{\| \prime}^{\mathrm{e}}} \mathrm{VR}^{\mathrm{e}}(\Omega) \mathrm{e}^{\tilde{\beta}_{\| \mathrm{A}_{\| \prime}}^{+\mathrm{e}}}\right| \psi^{\{\mathrm{C}\}_{j}} ; \mathrm{b}_{0}>^{\mathrm{e}}= \\
& =\frac{1}{6} \sum_{\left\{\mathrm{C}^{\prime}\right\}} \sum_{\{\mathrm{C}\}} \frac{1}{2} \sum_{\alpha \beta \gamma \delta}<\phi_{\alpha}^{\left\{\mathrm{C}^{\prime}\right\}} \phi_{\beta}^{\left\{\mathrm{C}^{\prime}\right\}}\left|\mathrm{V}_{12}\right| \tilde{\phi}_{\gamma}^{\{\mathrm{C}\}} \tilde{\phi}_{\delta}^{\{\mathrm{C}\}}>\mathrm{C}_{\alpha \beta \gamma \delta}^{\left\{\mathrm{C}^{\prime}, \mathrm{C}\right\}}
\end{aligned}
$$

onde na última passagem usamos a expressão (22) e $\mathrm{C}_{\alpha \beta \gamma \delta}^{\left\{\mathrm{C}^{\prime}, \mathrm{C}\right\}}$ indica o duplo cofator associado à matriz do overlap entre os estados determinantais: 


$$
\begin{aligned}
& \mathrm{e}^{\tilde{\beta}_{\| 1}^{\prime} \mathrm{A}_{\| 1}^{+\mathrm{e}}} \mid \psi^{\left\{\mathrm{C}^{\prime}\right\}} ; \mathrm{b}_{0}> \\
& \mathrm{R}^{\mathrm{e}}(\Omega) \mathrm{e}^{\tilde{\beta}_{\| \mathrm{A}_{11}^{+e}}^{+e} \mid \psi^{\{\mathrm{C}\}} ; \mathrm{b}_{0}>}
\end{aligned}
$$

e $\phi_{\alpha}^{\left\{\mathrm{C}^{\prime}\right\}}, \tilde{\phi}_{\gamma}^{\{\mathrm{C}\}}$ correspondem às funções de onda de partículas independentes associadas respectivamente aos estados (36) e (37) acima.

A interação de dois corpos $V_{12}$, dada pela expressão (21), não depende de spin e isospin e portanto podemos reescrever a expressão (35) como:

$$
\begin{aligned}
& { }^{\mathrm{e}}<0(62) ; \mathrm{b}_{0}\left|\mathrm{e}^{\tilde{\beta}_{\| 1}^{\prime} \mathrm{A}_{\| 1}^{\mathrm{e}}} \mathrm{VR}^{\mathrm{e}}(\Omega) \mathrm{e}^{\tilde{\beta}_{\| 1} \mathrm{~A}_{\| 1}^{+\mathrm{e}}}\right| 0(62) ; \mathrm{b}_{0}>^{\mathrm{e}}= \\
& =\frac{1}{6} \sum_{\left\{\mathrm{C}^{\prime}\right\}} \sum_{\left\{\mathrm{C}^{\prime}\right\}} \frac{1}{2} \sum_{\substack{\mathrm{a}, \mathrm{b} \\
\mathrm{i} j \mathrm{k} 1}}<\phi_{\mathrm{i} \mathrm{a}}^{\left\{\mathrm{C}^{\prime}\right\}} \phi_{\mathrm{jb}}^{\left\{\mathrm{C}^{\prime}\right\}}\left|\mathrm{V}_{12}\right| \tilde{\phi}_{\mathrm{ka}}^{\{\mathrm{C}\}} \tilde{\phi}_{1 \mathrm{~b}}^{\{\mathrm{C}\}}>\mathrm{C}_{\mathrm{i} a \mathrm{jb} k \mathrm{ka} 1 \mathrm{~b}}^{\left\{\mathrm{C}^{\prime}, \mathrm{C}\right\}} .
\end{aligned}
$$

Na seção A.1.2.2 classificamos os 36 termos associados ao overlap, eq.(13) em três grupos, onde a propriedade comum a cada elemento de um grupo era que ele possuia os mesmos blocos associados ao overlap entre estados espaciais. Os elementos de um mesmo grupo só diferem na ocupação dos estados de spin-isospin de cada bloco. Como a interação $\mathrm{V}_{12}$, eq.(21) não depende de spin e isospin é imediato que a contribuição ao potencial de todos os termos na eq.(38) que se originam de um mesmo grupo, obtido pela classificação do overlap, será idêntica. Logo podemos escrever a expressão (38) como:

$$
{ }^{\mathrm{e}}<0(62) ; \mathrm{b}_{0}\left|\mathrm{e}^{\tilde{\beta}_{\| \mathrm{A}_{\| \prime}^{\prime}}^{\mathrm{e}}} \mathrm{VR} \mathrm{R}^{\mathrm{e}}(\Omega) \mathrm{e}^{\tilde{\beta}_{\| \mathrm{A}_{\| 1}}^{+\mathrm{e}}}\right| 0(62) ; \mathrm{b}_{0}>^{\mathrm{e}}=\mathrm{I}_{1}+\mathrm{I}_{2}+\mathrm{I}_{3}
$$


onde $\mathrm{I}_{1}, \mathrm{I}_{2}, \mathrm{I}_{3}$ correspondem à contribuição dos grupos 1,2 e 3 respectivamente.

A contribuição devida aos seis termos do grupo 1, que obtivemos na seção A.1.2.2, é dada por:

$$
\mathrm{I}_{1}=\frac{1}{2} \sum_{\substack{\mathrm{a}, \mathrm{b} \\ \mathrm{i} j \mathrm{k} l}}<\phi_{\mathrm{ia}} \phi_{\mathrm{jb}}\left|\mathrm{V}_{12}\right| \tilde{\phi}_{\mathrm{ka}} \tilde{\phi}_{1 \mathrm{~b}}>\mathrm{C}_{\mathrm{ia}}^{(1)} \mathrm{jb} \mathrm{ka} \mathrm{lb}
$$

onde $\mathrm{C}_{\mathrm{ia}}^{(1)} \mathrm{jb}$ ka lb é o duplo cofator associado a:

$$
\mathrm{B}^{(1)}=\left[\begin{array}{ccccc}
\mathrm{B}_{11}^{\mathrm{n}+} & & & \\
& & & \\
& \mathrm{B}_{11}^{\mathrm{n}-} & & \\
& & & \\
& & \mathrm{B}_{22}^{\mathrm{p}+} & \\
& & & \mathrm{B}_{22}^{\mathrm{p}-}
\end{array}\right] \text {. }
$$

A contribuição devida aos seis termos do grupo 2 é dada por:

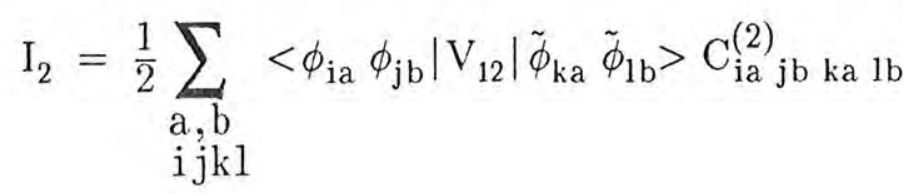

onde $\mathrm{C}_{\mathrm{ia}}^{(2)}{ }_{\mathrm{jb}}$ ka lb é o duplo cofator associado a: 


$$
\mathrm{B}^{(2)}=\left[\begin{array}{cccc}
\mathrm{B}_{12}^{\mathrm{n}+} & & & \\
& & & \\
& \mathrm{B}_{12}^{\mathrm{n}-} & & \\
& & & \\
& & \mathrm{B}_{21}^{\mathrm{p}} & \\
& & & \\
& & \mathrm{B}_{21}^{\mathrm{p}-}
\end{array}\right]
$$

e finalmente a contribuição devido aos 24 termos do grupo 3 é dada por:

$$
\mathrm{I}_{3}=4 \times \frac{1}{2} \sum_{\substack{\mathrm{a}, \mathrm{b} \\ \mathrm{ijk} 1}}<\phi_{\mathrm{ia}} \phi_{\mathrm{jb}}\left|\mathrm{V}_{12}\right| \tilde{\phi}_{\mathrm{ka}} \tilde{\phi}_{1 \mathrm{~b}}>\mathrm{C}_{\mathrm{ia} \mathrm{jb} \mathrm{ka} \mathrm{lb}}^{(3)}
$$

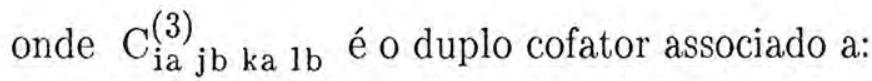

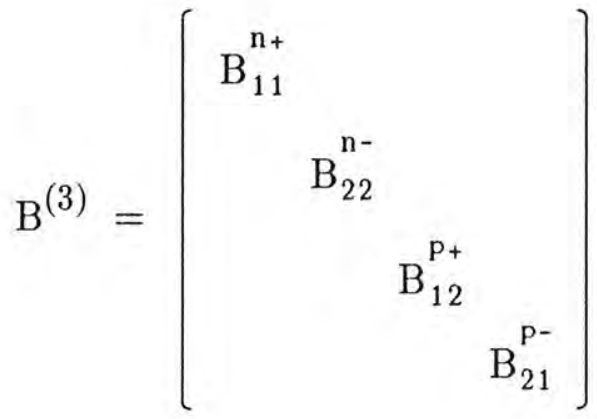

onde a notação usada é a mesma da seção A.1.2.2. Como mostramos na seção A.1.2.2, $\operatorname{det} \mathrm{B}_{12}=\operatorname{det} \mathrm{B}_{21}=0$ e portanto o termo $\mathrm{I}_{2}$ não vai contribuir uma vez que não existem duplocofatores não nulos neste caso. Logo temos apenas que nos preocupar com os termos $I_{1}$ e $I_{3}$ que faremos separadamente a seguir: 


\section{a) Cálculo de $I_{1}$}

Como da eq.(18), $\quad \operatorname{det} \mathrm{B}_{11} \neq 0$ e $\operatorname{det} \mathrm{B}_{22} \neq 0$, concluimos que $\operatorname{det} \mathrm{B}^{(1)} \neq 0$ e portanto podemos usar a expressão (23) neste caso, onde obtemos:

$$
\begin{aligned}
& I_{1}=\frac{1}{2} \operatorname{det}^{2} B_{11} \operatorname{det}^{2} B_{22} . \\
& \cdot \sum_{\substack{a, b \\
i j k 1}}<\phi_{i a} \phi_{j b}\left|V_{12}\right| \tilde{\phi}_{k a} \tilde{\phi}_{1 b}>\left[\left[B^{(1)}\right]_{k a \text { ia }}^{-1}\left[B^{(1)}\right]_{1 b ~ j b}^{-1}-\left[B^{(1)}\right]_{k a ~ j b}^{-1}\left[B^{(1)}\right]_{1 b \text { ia }}^{-1}\right]
\end{aligned}
$$

e notando a estrutura $\mathrm{B}^{(1)}$ dada pela expressão (40), podemos somar nos rótulos de spin-isospin obtendo:

$$
\begin{aligned}
& \mathrm{I}_{2}=\operatorname{det}^{2} \mathrm{~B}_{11} \operatorname{det}^{2} \mathrm{~B}_{22} \cdot \\
& \cdot\left\{\sum_{\mathrm{i}_{1} \mathrm{j}_{1} \mathrm{k}_{1} 1_{1}}\left[\mathrm{~V}_{\mathrm{D}}^{(1)}<\phi_{\mathrm{i}_{1}} \phi_{\mathrm{j}_{1}}|\mathrm{u}| \tilde{\phi}_{\mathrm{k}_{1}} \tilde{\phi}_{1_{1}}>+\mathrm{V}_{\mathrm{E}}^{(1)}<\phi_{\mathrm{i}_{1}} \phi_{\mathrm{j}_{1}}|\mathrm{u}| \tilde{\phi}_{1_{1}} \tilde{\phi}_{\mathrm{k}_{1}}>\right]\left[\mathrm{B}_{11}^{-1}\right] \mathrm{k}_{1_{1} \mathrm{i}_{1}}\left[\mathrm{~B}_{11}^{-1}\right]_{1_{1} \mathrm{j}_{1}}+\right. \\
& +\sum_{\mathrm{i}_{2} \mathrm{j}_{2} \mathrm{k}_{2} \mathrm{l}_{2}}\left[\mathrm{~V}_{\mathrm{D}}^{(1)}<\phi_{\mathrm{i}_{2}} \phi_{\mathrm{j}_{2}}|\mathrm{u}| \tilde{\phi}_{\mathrm{k}_{2}} \tilde{\phi}_{1_{2}}>+\mathrm{V}_{\mathrm{E}}^{(1)}<\phi_{\mathrm{i}_{2}} \phi_{\mathrm{j}_{2}}|\mathrm{u}| \tilde{\phi}_{1_{2}} \tilde{\phi}_{\mathrm{k}_{2}}>\right]\left[\mathrm{B}_{22}^{-1}\right] \mathrm{k}_{2} \mathrm{i}_{2}\left[\mathrm{~B}_{22}^{-1}\right]_{1_{2} \mathrm{j}_{2}}+ \\
& +\sum_{\mathrm{i}_{1} \mathrm{j}_{2}}\left[\mathrm{~V}_{\mathrm{D}}^{(2)}<\phi_{\mathrm{i}_{1}} \phi_{\mathrm{j}_{2}}|\mathrm{u}| \tilde{\phi}_{\mathrm{k}_{1}} \tilde{\phi}_{1_{2}}>+\mathrm{V}_{\mathrm{E}}^{(2)}<\phi_{\mathrm{i}_{1}} \phi_{\mathrm{j}_{2}}|\mathrm{u}| \tilde{\phi}_{1_{2}} \tilde{\phi}_{\mathrm{k}_{1}}>\right]\left[\mathrm{B}_{11}^{-1}\right] \mathrm{k}_{1_{1} \mathrm{i}_{1}}\left[\mathrm{~B}_{22}^{-1} \mathrm{l}_{2} \mathrm{j}_{2}\right] \\
& \left.\mathrm{k}_{1} \mathrm{l}_{2}\right]
\end{aligned}
$$

onde definimos 


$$
\begin{array}{ll}
\mathrm{V}_{\mathrm{D}}^{(1)}=2 \mathrm{~V}_{0}-\mathrm{V}_{\mathrm{M}} & \mathrm{V}_{\mathrm{E}}^{(1)}=-\mathrm{V}_{0}+2 \mathrm{~V}_{\mathrm{M}} \\
\mathrm{V}_{\mathrm{D}}^{(2)}=4 \mathrm{~V}_{0} & \mathrm{~V}_{\mathrm{E}}^{(2)}=4 \mathrm{~V}_{\mathrm{M}} .
\end{array}
$$

A expressão (43) é calculada de modo análogo à seção A.1.3.3.

\section{b) Cálculo do Termo $\mathrm{I}_{3}$}

Como vimos na seção A.1.2.2, $\operatorname{det} \mathrm{B}_{12}=\operatorname{det} \mathrm{B}_{21}=0$ e portanto o determinante de $\mathrm{B}^{(3)}$, dado pela eq.(42) é nulo, assim não podemos usar o mesmo método usado no caso (a).

Vamos obter a seguir uma expressão apropriada para o cálculo neste caso. Como em (42) $\operatorname{det} \mathrm{B}_{12}=\operatorname{det} \mathrm{B}_{21}=0$, somente duplo-cofatores com rótulos de spin-isospin $a, b$, na eq.(41) associados aos blocos $\mathrm{B}_{12}^{\mathrm{p+}}$ e $\mathrm{B}_{21}^{\mathrm{p}^{-}}$de $\mathrm{B}^{(3)}$, poderão gerar contribuição não nulas. Levando em conta a estrutura $\mathrm{B}^{(3)}$ e o fato de $\mathrm{V}$ não depender de spin isospin obtemos

$$
\mathrm{I}_{3}=\sum_{\mathrm{i}_{1} \mathrm{j}_{2} \mathrm{k}_{2} \mathrm{l}_{1}}\left[4 \mathrm{~V}_{0}<\phi_{\mathrm{i}_{1}} \phi_{\mathrm{j}_{2}}|\mathrm{u}| \tilde{\phi}_{\mathrm{k}_{2}} \tilde{\phi}_{\mathrm{l}_{1}}>+4 \mathrm{~V}_{\mathrm{M}}<\phi_{\mathrm{i}_{1}} \phi_{\mathrm{j}_{2}}|\mathrm{u}| \tilde{\phi}_{\mathrm{l}_{1}} \tilde{\phi}_{\mathrm{k}_{2}}>\right] \mathrm{C}_{\mathrm{i}_{1} \mathrm{j}_{2} \mathrm{k}_{2} \mathrm{l}_{1}}
$$

onde o duplo-cofator $\mathrm{C}_{\mathrm{i}_{1} \mathrm{j}_{2} \mathrm{k}_{2} \mathrm{l}_{1}}$ é dado abaixo: 


$$
\mathrm{C}_{\mathrm{i}_{1} \mathrm{j}_{2} \mathrm{k}_{2} \mathrm{l}_{1}}=\operatorname{det}\left[\begin{array}{cccc}
\mathrm{B}_{11} & & & \\
& \mathrm{~B}_{22} & & \\
& \mathrm{i}_{1} \frac{\phi_{12}}{} & \\
& \mathrm{k}_{2} & \\
& \mathrm{j}_{2}-\mathrm{\beta}_{21} \\
& & \mathrm{l}_{1}
\end{array}\right]
$$

$\mathrm{Na}$ expressão acima as linhas horizontais e verticais cortando $\mathrm{B}_{12}$ e $\mathrm{B}_{21}$ indicam que na matriz $B_{12}$ substituimos os elementos da linha $i_{1}$ e da coluna $k_{2}$ por zero a menos do elemento $\left(\mathrm{i}_{1}, \mathrm{k}_{2}\right)$ que substituimos pelo número 1 e de modo análogo substituimos os elementos de $\mathrm{B}_{21}$. Recordemos que este é o algaritmo que nos permite calcular o duplo cofator.

A partir do cálculo realizado na seção A.1.2.2 para os elementos de matriz do overlap e do procedimento descrito na seção anterior podemos calcular a expressão (39) e obter os elementos de matriz de $\mathrm{V}$ através do MFG.

\section{A.1.3.5 -Cálculo do Potencial na Representação 4p-4b}

Todo o procedimento deste caso é completamente análogo ao usado na seção A.1.3.1 e portanto não vamos analisá-lo. 


\section{Apêndice 2 - Forma Normal dos Operadores de Interesse}

Neste apêndice, vamos mostrar a técnica que possibilita obter a forma normal dos operadores pertencentes ao grupo $\mathrm{Sp}(3, \mathrm{R})$ e que sistematicamente utilizamos no cálculo dos elementos de matriz dos operadores de interesse.

\section{A.2.1 - Forma Normal de Um Operador do Grupo $\mathrm{Sp}(3, \mathrm{R})$}

Definimos a forma normal de um operador do grupo $\mathrm{Sp}(3, \mathrm{R})$ como sendo a sua representação onde a direita deixamos apenas termos envolvendo os abaixadores da álgebra, a esquerda, termos que envolvem apenas os levantadores e entre estes, termos envolvendo os operadores de peso.

A seguir vamos obter a representação matricial de um operador arbitrário de $\mathrm{Sp}(3, \mathrm{R})$ que, como veremos, será adequada para calcularmos a sua forma normal. Definimos $\mathrm{a}_{\mathrm{i}}^{+}, \mathrm{a}_{\mathrm{j}}$ pelas relações de comutação seguintes:

$$
\begin{aligned}
& {\left[\mathrm{a}_{\mathrm{i}}^{+}, \mathrm{a}_{\mathrm{j}}^{+}\right]=\left[\mathrm{a}_{\mathrm{i}}, \mathrm{a}_{\mathrm{j}}\right]=0 \quad \mathrm{i}, \mathrm{j}=1,2,3} \\
& {\left[\mathrm{a}_{\mathrm{i}}, \mathrm{a}_{\mathrm{j}}^{+}\right]=\delta_{\mathrm{ij}}}
\end{aligned}
$$

onde a realização dos operadores $a_{i}, a_{j}^{+}$é completamente irrelevante para os desenvolvimentos que iremos fazer neste apêndice, isto é, nos basearemos exclusivamente nas propriedades algébricas de $a_{i}^{+}, a_{i}$ dadas acima.

A ação da trasformação $\mathrm{e}^{\mathrm{W}}$ onde $\mathrm{W}$ envolve apenas o produto bilinear dos operadores $a_{i}^{+}, a_{j}$, nos operadores $a_{i}^{+}, a_{i}$ é dada por: 


$$
\begin{aligned}
& a_{i}^{+}=e^{W} a_{i}^{+} e^{-W}=\sum_{j} a_{j}^{+} A_{j i}+a_{j} C_{j i} \\
& a_{i}^{\prime}=e^{W} a_{i} e^{-W}=\sum_{j} a_{j}^{+} B_{j i}+a_{j} D_{j i} .
\end{aligned}
$$

A transformação $e^{W}$ deixa invariante os comutadores definidos pela eq.(1) e é denomindada simplética.

Um elemento arbitrário $\underset{\sim}{\sigma}$ do espaço vetorial gerado pela base $a_{i}^{+}, a_{i}$, é dado por:

$$
\underset{\sim}{\sigma}=\sum_{i}\left(y_{i} a_{i}^{+}+z_{i} a_{i}\right)
$$

A ação da transformação $\mathrm{e}^{\mathrm{W}}$ no vetor acima é dada por:

$$
\begin{aligned}
\underset{\sim}{\sigma^{\prime}} & =e^{W} \underset{\sim}{\sigma} e^{-W}=\sum_{i, j}\left(A_{j i} y_{i}+B_{j i} z_{i}\right) a_{j}^{+}+\left(C_{j i} y_{i}+D_{j i} z_{i}\right) a_{j}= \\
& =\sum_{j}\left(y_{j}^{\prime} a_{j}^{+}+z_{j}^{\prime} a_{j}\right) .
\end{aligned}
$$

Podemos representar a transformação acima na forma matricial seguinte:

$$
\left[\begin{array}{c}
\check{\sim}^{\prime} \\
{\underset{\sim}{z}}^{\prime}
\end{array}\right]=\left[\begin{array}{ll}
\mathrm{A} & \mathrm{B} \\
\mathrm{C} & \mathrm{D}
\end{array}\right]\left[\begin{array}{c}
\underset{\sim}{\mathrm{y}} \\
\underset{\sim}{z}
\end{array}\right]
$$

onde os elementos de matriz de A, B, C, D são dados em (4) e usamos a notação: 


$$
\underset{\sim}{\mathrm{y}}=\left[\begin{array}{l}
\mathrm{y}_{1} \\
\mathrm{y}_{2} \\
\mathrm{y}_{3}
\end{array}\right] \text {. }
$$

A partir das relações de comutação entre $a_{i}^{+1}$ e $a_{i}^{\prime}$, que são as mesmas que definimos em (1), obtemos:

$$
\begin{aligned}
& {\left[a_{i}^{+1}, a_{j}^{+1}\right]=0 \rightarrow \sum_{1} C_{1 i} A_{1 j}-A_{1 i} C_{1 j}=0} \\
& {\left[a_{i}^{\prime}, a_{j}^{\prime}\right]=0 \rightarrow \sum_{l} D_{1 i} B_{1 j}-B_{1 i} D_{1 j}=0} \\
& {\left[a_{i}^{\prime}, a_{j}^{+1}\right]=\delta_{i j} \rightarrow \sum_{1} D_{1 i} A_{1 j}-B_{1 i} C_{1 j}=\delta_{i j} \cdot}
\end{aligned}
$$

As relações acima podem ser reescritas em forma matricial, e assim obtemos:
a) $\mathrm{C}^{\mathrm{t}} \mathrm{A}=\mathrm{A}^{\mathrm{t}} \mathrm{C}$
b) $D^{t} B=B^{t} D$
c) $\mathrm{D}^{\mathrm{t}} \mathrm{A}-\mathrm{B}^{\mathrm{t}} \mathrm{C}=\mathrm{I}$

as condições acima devem ser satisfeitas para a transformação (4) ser simplética. A igualdade (c) pode ser reescrita do modo seguinte:

$$
A=\left(D^{t}\right)^{-1}+\left(D^{t}\right)^{-1} B^{t} C
$$


e como (b) implica que $\mathrm{B}^{\mathrm{t}}=\mathrm{D}^{\mathrm{t}} \mathrm{B} \mathrm{D}^{-1}$ obtemos:

$$
A=\left(D^{t}\right)^{-1}+B D^{-1} C
$$

e portanto concluimos que a matriz arbitrária que representa um elemento de $\operatorname{Sp}(3, \mathrm{R})$ pode ser escrita da forma seguinte:

$$
\left[\begin{array}{ll}
\mathrm{A} & \mathrm{B} \\
\mathrm{C} & \mathrm{D}
\end{array}\right]=\left[\begin{array}{cc}
\mathrm{I} & \mathrm{B} \mathrm{D}^{-1} \\
0 & \mathrm{I}
\end{array}\right]\left[\begin{array}{cc}
\left(\mathrm{D}^{\mathrm{t}}\right)^{-1} & 0 \\
0 & \mathrm{D}
\end{array}\right]\left[\begin{array}{cc}
\mathrm{I} & 0 \\
\mathrm{D}^{-1} \mathrm{C} & \mathrm{I}
\end{array}\right]
$$

onde I é a matriz identidade $3 \times 3$.

Vamos agora identificar as transformações de $\mathrm{Sp}(3, \mathrm{R})$ associadas ao lado direito da eq.(8). Vamos primeiro analisar a ação da transformação

$$
e^{W}=e^{\sum_{i j} \lambda_{i j} A_{i j}^{+}} \text {, onde } A_{i j}^{+}=\frac{1}{2} a_{i}^{+} a_{j}^{+}
$$

no vetor $\underset{\sim}{\sigma}$, que definimos na eq.(3).

A partir da expressão bastante conhecida:

$$
\mathrm{e}^{\mathrm{B}} \mathrm{Ae}^{-\mathrm{B}}=\mathrm{A}+[\mathrm{A}, \mathrm{B}]+\frac{1}{2 !}[\mathrm{B},[\mathrm{B}, \mathrm{A}]]+\frac{1}{3 !}[\mathrm{B},[\mathrm{B},[\mathrm{B}, \mathrm{A}]]]+\ldots
$$

e das relações de comutação abaixo, que obtemos pelo cálculo direto: 


$$
\begin{aligned}
& {\left[\mathrm{A}_{\mathrm{ij}}^{+}, \mathrm{a}_{\mathrm{l}}^{+}\right]=0} \\
& {\left[\mathrm{~A}_{\mathrm{ij}}^{+}, \mathrm{a}_{1}\right]=-\frac{1}{2}\left(\delta_{1 \mathrm{j}} \mathrm{a}_{\mathrm{i}}^{+}+\delta_{1 \mathrm{i}} \mathrm{a}_{\mathrm{j}}^{+}\right)}
\end{aligned}
$$

segue que:

$$
{\underset{\sim}{\sigma}}^{\prime}=\mathrm{e}^{\sum_{\mathrm{ij}} \lambda_{\mathrm{ij}} \mathrm{A}_{\mathrm{ij}}^{+}} \underset{\sim}{\sigma} \mathrm{e}^{-\sum_{\mathrm{ij}} \lambda_{\mathrm{ij}} \mathrm{A}_{\mathrm{ij}}^{+}}=\sum_{\mathrm{l}}\left[\sum_{\mathrm{k}} \delta_{1 \mathrm{k}} \mathrm{y}_{\mathrm{k}}-\lambda_{\mathrm{lk}} \mathrm{z}_{\mathrm{k}}\right] \mathrm{a}_{\mathrm{l}}^{+}+\mathrm{z}_{1} \mathrm{a}_{\mathrm{l}}
$$

e portanto comparando com a eq.(4), segue que a representação matricial da transformação $\mathrm{e}^{\mathrm{W}}$, dada pela eq.(9), é a seguinte:

$$
\left[\begin{array}{l}
{\underset{\sim}{\prime}}^{\prime} \\
\underset{\sim}{z^{\prime}}
\end{array}\right]=\left[\begin{array}{cc}
\mathrm{I} & -\underset{\sim}{\lambda} \\
0 & \mathrm{I}
\end{array}\right]\left[\begin{array}{c}
\underset{\sim}{\mathrm{y}} \\
\underset{\sim}{z}
\end{array}\right]
$$

onde supomos sem perda de generalidade que $(\underset{\sim}{\lambda})_{\mathrm{ij}}=(\underset{\sim}{\lambda})_{\mathrm{ji}} \operatorname{com}(\underset{\sim}{\lambda})_{\mathrm{ij}}=\lambda_{\mathrm{ij}}$. De maneira semelhante podemos mostrar que a ação de

$$
e^{W}=e^{\sum_{i j} \lambda_{i j} A_{i j}}, \text { onde } A_{i j}=\frac{1}{2} a_{i} a_{j}
$$

no vetor $\underset{\sim}{\sigma}$ é dada por: 


$$
\tilde{\sigma}^{\prime}=\mathrm{e}^{\sum_{\mathrm{ij}} \lambda_{\mathrm{ij}} \mathrm{A}_{\mathrm{ij}}} \underset{\sim}{\sigma} \mathrm{e}^{-\sum_{\mathrm{ij}} \lambda_{\mathrm{ij}} \mathrm{A}_{\mathrm{ij}}}=\sum_{1} \mathrm{y}_{1} \mathrm{a}_{1}^{+}+\left[\sum_{\mathrm{k}} \lambda_{1 \mathrm{k}} \mathrm{y}_{\mathrm{k}}+\delta_{1 \mathrm{k}} \mathrm{z}_{\mathrm{k}}\right] \mathrm{a}_{\mathrm{l}}
$$

e portanto a transformação $\mathrm{e}^{\mathrm{W}}$, eq.(13) tem a seguinte representação matricial:

$$
\left[\begin{array}{c}
\tilde{\sim}^{\prime} \\
z_{\sim}^{\prime}
\end{array}\right]=\left[\begin{array}{ll}
\mathrm{I} & 0 \\
\lambda & \mathrm{I}
\end{array}\right]\left[\begin{array}{c}
\underset{\sim}{\mathrm{y}} \\
\underset{\sim}{z}
\end{array}\right]
$$

e finalmente de modo análogo, temos que a ação de

$$
\mathrm{e}^{\mathrm{W}}=\mathrm{e}^{\sum_{\mathrm{ij}} \alpha_{\mathrm{ij}} \mathrm{C}_{\mathrm{ij}}} \text {, onde } \mathrm{C}_{\mathrm{ij}}=\frac{1}{2}\left(\mathrm{a}_{\mathrm{i}}^{+} \mathrm{a}_{\mathrm{j}}+\mathrm{a}_{\mathrm{j}} \mathrm{a}_{\mathrm{i}}^{+}\right)
$$

no vetor $\underset{\sim}{\sigma}$ é dada por:

$$
\begin{aligned}
\stackrel{\sim}{\prime}^{\prime} & =e^{\sum_{\mathrm{ij}} \alpha_{\mathrm{ij}} \mathrm{C}_{\mathrm{ij}}} \underset{\sim}{\sigma} \mathrm{e}^{-\sum_{\mathrm{ij}} \alpha_{\mathrm{ij}} \mathrm{C}_{\mathrm{ij}}}=\sum_{1}\left[\sum_{\mathrm{k}}\left(\mathrm{e}^{\alpha}\right)_{1 \mathrm{k}} \mathrm{y}_{\mathrm{k}}\right] \mathrm{a}_{1}^{+}+ \\
& +\left[\sum_{\mathrm{k}}\left(\mathrm{e}^{-}{ }^{\mathrm{t}}\right)_{1 \mathrm{k}}^{\mathrm{t}} \mathrm{z}_{\mathrm{k}}\right] \mathrm{a}_{\mathrm{l}}
\end{aligned}
$$

e portanto a transformação $\mathrm{e}^{\mathrm{W}}$, eq.(15) tem a seguinte representação matricial:

$$
\left[\begin{array}{c}
\underset{\sim}{y^{\prime}} \\
\underset{\sim}{z^{\prime}}
\end{array}\right]=\left[\begin{array}{cc}
\mathrm{e}^{\alpha} & 0 \\
0 & \mathrm{e}^{-\alpha^{\mathrm{t}}}
\end{array}\right]\left[\begin{array}{c}
\underset{\sim}{\mathrm{y}} \\
\underset{\sim}{z}
\end{array}\right]
$$


onde $(\alpha)_{\mathrm{ij}}=\alpha_{\mathrm{ij}}$.

Comparando a expressão (8) com (12),(14) e (16), obtemos que uma transformação simplética arbitrária, representada pela expressão (5), pode ser escrita na forma:

$$
e^{W}=e^{\sum_{i j} h_{i j} A_{i j}^{+}} e^{\sum_{i j} \alpha_{i j} C_{i j}} e^{\sum_{i j} h_{i j}^{\prime} A_{i j}}
$$

onde

$$
\begin{aligned}
& \mathrm{h}_{\mathrm{ij}}=\mathrm{h}_{\mathrm{ji}}=-\left(\mathrm{B} \mathrm{D}^{-1}\right)_{\mathrm{ij}} \\
& \mathrm{h}_{\mathrm{ij}}^{\prime}=\mathrm{h}_{\mathrm{ji}}^{\prime}=\left(\mathrm{D}^{-1} \mathrm{C}\right)_{\mathrm{ij}} \\
& \left(\mathrm{e}^{\alpha}\right)_{\mathrm{ij}}=\left(\mathrm{D}^{+}\right)_{\mathrm{ij}}^{-1} .
\end{aligned}
$$

As matrizes h e h' são simétricas, como é fácil ver das relações (6).

Para completarmos a tarefa de obter a forma normal do operador $\mathrm{e}^{\mathrm{W}}$, precisamos apenas escrever a transformação (16) em sua forma normal, isto é:

$$
e^{\sum_{i j} \alpha_{i j} C_{i j}}=\prod_{i>j}\left[e^{\bar{\alpha}_{i j} C_{i j}}\right] e^{\sum_{i} \bar{\alpha}_{i i} C_{i i}} \prod_{i<j}\left[e^{\bar{\alpha}_{i j} C_{i j}}\right] .
$$


A representação matricial do operador $\alpha_{\mathrm{k} 1} \mathrm{C}_{\mathbf{k} 1}$, é obtida facilmente da expressão (16),por exemplo:

$$
\underset{\sim}{\sigma^{\prime}}=\mathrm{e}^{\alpha_{12} \mathrm{C}_{12}} \underset{\sim}{\sigma} \mathrm{e}^{-\alpha_{12} \mathrm{C}_{12}}
$$

e portanto a representação matricial de $\alpha_{12} \mathrm{C}_{12}$ é dada por:

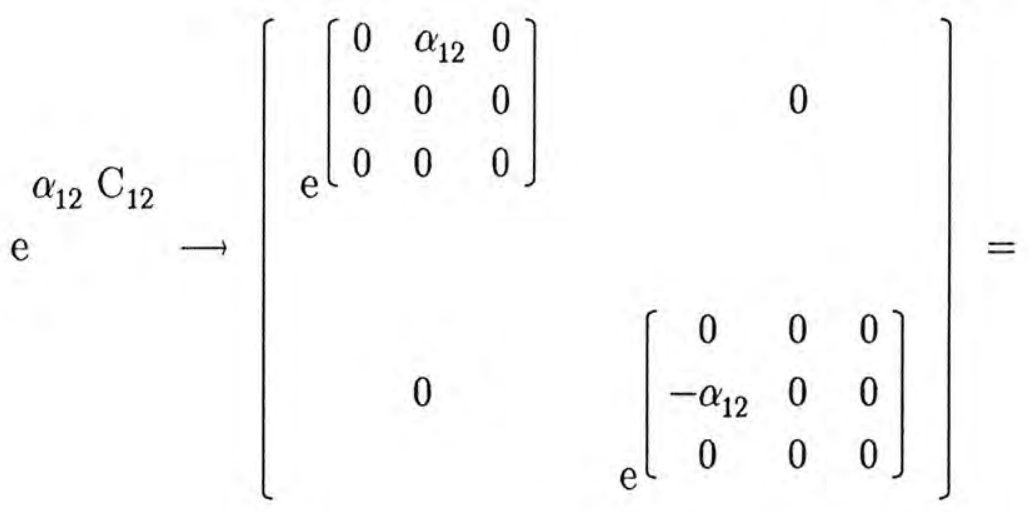

$$
\begin{aligned}
& =\left[\begin{array}{ccccccc}
1 & \alpha_{12} & 0 & & & \\
0 & 1 & 0 & & 0 & \\
0 & 0 & 1 & & & \\
& & & 1 & 0 & 0 \\
& & & -\alpha_{12} & 1 & 0 \\
& 0 & & 0 & 0 & 1
\end{array}\right]
\end{aligned}
$$

e de maneira análoga obtemos a representação matricial de qualquer $\alpha_{\mathrm{k} 1} \mathrm{C}_{\mathrm{k} 1}$.

A fim de simplificarmos a notação, vamos analisar apenas a parte relativa a $e^{\alpha}$ na eq.(16) ficando sub-entendido a parte $\mathrm{e}^{-\alpha^{\mathrm{t}}}$. 
Definindo

$$
\mathrm{e}^{\alpha}=\left[\begin{array}{lll}
\mathrm{g}_{11} & \mathrm{~g}_{12} & \mathrm{~g}_{13} \\
\mathrm{~g}_{21} & \mathrm{~g}_{22} & \mathrm{~g}_{23} \\
\mathrm{~g}_{31} & \mathrm{~g}_{32} & \mathrm{~g}_{33}
\end{array}\right]
$$

segue que a matriz acima pode ser escrita da maneira seguinte:

$$
\mathrm{e}^{\alpha}=\left[\begin{array}{lll}
\mathrm{g}_{11} & \mathrm{~g}_{12} & \mathrm{~g}_{13} \\
\mathrm{~g}_{21} & \mathrm{~g}_{22} & \mathrm{~g}_{23} \\
\mathrm{~g}_{31} & \mathrm{~g}_{32} & \mathrm{~g}_{33}
\end{array}\right]=\left[\begin{array}{lll}
1 & 0 & 0 \\
\bar{\alpha}_{21} & 1 & 0 \\
\bar{\alpha}_{31} & \bar{\alpha}_{32} & 1
\end{array}\right]\left[\begin{array}{lll}
\mathrm{e}^{\bar{\alpha}_{11}} & 0 & 0 \\
0 & \mathrm{e}^{\bar{\alpha}_{22}} & 0 \\
0 & 0 & \mathrm{e}^{\bar{\alpha}_{33}}
\end{array}\right]\left[\begin{array}{lll}
1 & \bar{\alpha}_{12} & \bar{\alpha}_{13} \\
0 & 1 & \bar{\alpha}_{23} \\
0 & 0 & 1
\end{array}\right]
$$

onde definimos:

$$
\begin{gathered}
\mathrm{e}^{\bar{\alpha}_{11}=\mathrm{g}_{11},} \quad \mathrm{e}^{\bar{\alpha}_{22}}=\frac{\mathrm{g}_{11} \mathrm{~g}_{22}-\mathrm{g}_{12} \mathrm{~g}_{21}}{\mathrm{~g}_{11}}, \quad \mathrm{e}^{\bar{\alpha}_{33}}=\frac{\operatorname{det} \mathrm{g}}{\mathrm{g}_{11} \mathrm{~g}_{22}-\mathrm{g}_{12} \mathrm{~g}_{21}} \\
\bar{\alpha}_{12}=\frac{\mathrm{g}_{12}}{\mathrm{~g}_{11}}, \quad \bar{\alpha}_{13}=\frac{\mathrm{g}_{13}}{\mathrm{~g}_{11}}, \quad \bar{\alpha}_{23}=\frac{\mathrm{g}_{11} \mathrm{~g}_{23}-\mathrm{g}_{13} \mathrm{~g}_{21}}{\mathrm{~g}_{11} \mathrm{~g}_{22}-\mathrm{g}_{12} \mathrm{~g}_{21}} \\
\bar{\alpha}_{21}=\frac{\mathrm{g}_{21}}{\mathrm{~g}_{11}} \quad, \quad \bar{\alpha}_{31}=\frac{\mathrm{g}_{31}}{\mathrm{~g}_{11}}, \quad \bar{\alpha}_{32}=\frac{\mathrm{g}_{11} \mathrm{~g}_{32}-\mathrm{g}_{31} \mathrm{~g}_{12}}{\mathrm{~g}_{11} \mathrm{~g}_{22}-\mathrm{g}_{12} \mathrm{~g}_{21}}
\end{gathered}
$$

a decomposição (19) pode ser verificada pela substituição direta. 
Através da substituição direta da representação matricial dos operadores $\mathrm{C}_{32}, \mathrm{C}_{12}, \mathrm{C}_{13}$ obtemos:

$$
\begin{aligned}
\mathrm{e}^{\bar{\alpha}_{23} \mathrm{C}_{23}} \mathrm{e}^{\bar{\alpha}_{12} \mathrm{C}_{12}} \mathrm{e}^{\bar{\alpha}_{13} \mathrm{C}_{13}} & =\left[\begin{array}{lll}
1 & 0 & 0 \\
0 & 1 & \bar{\alpha}_{23} \\
0 & 0 & 1
\end{array}\right]\left[\begin{array}{lll}
1 & \bar{\alpha}_{12} & 0 \\
0 & 1 & 0 \\
0 & 0 & 1
\end{array}\right]\left[\begin{array}{lll}
1 & 0 & \bar{\alpha}_{13} \\
0 & 1 & 0 \\
0 & 0 & 1
\end{array}\right]= \\
& =\left[\begin{array}{lll}
1 & \bar{\alpha}_{12} & \bar{\alpha}_{13} \\
0 & 1 & \bar{\alpha}_{23} \\
0 & 0 & 1
\end{array}\right]
\end{aligned}
$$

e analogamente obtemos:

$$
\begin{aligned}
\mathrm{e}^{\bar{\alpha}_{31} \mathrm{C}_{31}} \mathrm{e}^{\bar{\alpha}_{21} \mathrm{C}_{21}} \mathrm{e}^{\bar{\alpha}_{32} \mathrm{C}_{32}}=\left[\begin{array}{ccc}
1 & 0 & 0 \\
\bar{\alpha}_{21} & 1 & 0 \\
\bar{\alpha}_{31} & \bar{\alpha}_{32} & 1
\end{array}\right] \\
\mathrm{e}^{\bar{\alpha}_{11} \mathrm{C}_{11}} \mathrm{e}^{\bar{\alpha}_{22} \mathrm{C}_{22}} \mathrm{e}^{\bar{\alpha}_{33} \mathrm{C}_{33}}=\left[\begin{array}{lll}
\mathrm{e}^{\bar{\alpha}_{11}} & 0 \\
& \mathrm{e}^{\bar{\alpha}_{22}} & \\
0 & & \mathrm{e}^{\bar{\alpha}_{33}}
\end{array}\right] .
\end{aligned}
$$

Comparando (20), (21) e (22) com (19) obtemos a forma normal de (16) e junto com (17) a forma normal de um operador de $\mathrm{Sp}(3, \mathrm{R})$. 
Em resumo, se $\mathrm{e}^{\mathrm{W}}$ corresponde a uma transformação simplética arbitrária, representada na forma matricial pela eq.(5), isto é:

$$
\mathrm{e}^{\mathrm{W}} \rightarrow\left[\begin{array}{ll}
\mathrm{A} & \mathrm{B} \\
\mathrm{C} & \mathrm{D}
\end{array}\right]
$$

então sua forma normal é dada por:

$$
\begin{gathered}
\mathrm{e}^{\mathrm{W}}=\mathrm{e}^{\sum_{\mathrm{ij}} \mathrm{h}_{\mathrm{ij}} \mathrm{A}_{\mathrm{ij}}^{+}} \mathrm{e}^{\bar{\alpha}_{31} \mathrm{C}_{31}} \mathrm{e}^{\bar{\alpha}_{21} \mathrm{C}_{21}} \mathrm{e}^{\bar{\alpha}_{32} \mathrm{C}_{32}} \mathrm{e}^{\sum_{\mathrm{i}} \bar{\alpha}_{\mathrm{ii}} \mathrm{C}_{\mathrm{ii}}} . \\
\cdot \mathrm{e}^{\bar{\alpha}_{23} \mathrm{C}_{23}} \mathrm{e}^{\bar{\alpha}_{12} \mathrm{C}_{12}} \mathrm{e}^{\bar{\alpha}_{13} \mathrm{C}_{13}} \mathrm{e}^{\sum_{\mathrm{ij}} \mathrm{h}_{\mathrm{ij}} \mathrm{A}_{\mathrm{ij}}}
\end{gathered}
$$

onde

$$
\begin{aligned}
& h_{i j}=h_{j i}=-\left(B D^{-1}\right)_{i j} \\
& h_{i j}^{\prime}=h_{j i}^{\prime}=\left(D^{-1} C\right)_{i j}
\end{aligned}
$$

e $\bar{\alpha}_{\mathrm{ij}}$ são obtidos pelas expressões (19) onde identificamos:

$$
g_{i j}=\left(D^{t}\right)_{i j}^{-1}
$$

A seguir vamos obter a representação matricial dos operadores de interesse a fim de obtermos a sua forma normal pelo procedimento descrito acima. 


\section{A.2.2 - Represetação dos Operadores de Interesse}

\section{A.2.2.1 - Operador de Rotação}

$$
\mathrm{R}(\Omega)=\mathrm{R}_{\mathrm{z}}(\alpha) \mathrm{R}_{\mathrm{y}}(\beta) \mathrm{R}_{\mathrm{z}}(\gamma)
$$

onde

$$
\begin{aligned}
& \mathrm{R}_{\mathrm{z}}(\alpha)=\mathrm{e}^{-\mathrm{i} \alpha \mathrm{L}_{\mathrm{z}}} \\
& \mathrm{R}_{\mathrm{y}}(\beta)=\mathrm{e}^{-\mathrm{i} \beta \mathrm{L}_{\mathrm{y}}}
\end{aligned}
$$

é fácil mostrar que

$$
\begin{aligned}
& \mathrm{i} \mathrm{L}_{\mathrm{z}}=\mathrm{C}_{23}-\mathrm{C}_{32} \\
& \mathrm{i} \mathrm{L}_{\mathrm{y}}=\mathrm{C}_{12}-\mathrm{C}_{21} \quad(\hbar=1)
\end{aligned}
$$

usando a expressão (10) e as relações acima podemos mostrar que a ação de $R_{z}(\alpha)$ no vetor $\underset{\sim}{\sigma}$ é dada por:

$$
\begin{aligned}
\underset{\sim}{\sigma^{\prime}} & =\mathrm{e}^{-\mathrm{i} \alpha \mathrm{L}_{\mathrm{z}}} \underset{\sim}{\sigma} \mathrm{e}^{\mathrm{i} \alpha \mathrm{L}_{\mathrm{z}}}=\mathrm{e}^{-\gamma\left(\mathrm{C}_{23}-\mathrm{C}_{32}\right)} \underset{\sim}{\sigma} \mathrm{e}^{\gamma\left(\mathrm{C}_{23}-\mathrm{C}_{32}\right)}= \\
& =\sum_{\mathrm{k}, 1}\left(\mathrm{R}_{\mathrm{z}}(\alpha)\right)_{\mathrm{kl}} \mathrm{y}_{1} \mathrm{a}_{\mathrm{k}}^{+}+\left(\mathrm{R}_{\mathrm{z}}(\alpha)\right)_{\mathrm{kl}} \mathrm{z}_{1} \mathrm{a}_{\mathrm{k}}
\end{aligned}
$$


onde

$$
\mathrm{R}_{\mathrm{z}}(\alpha)=\left[\begin{array}{ccc}
1 & 0 & 0 \\
0 & \cos \alpha & -\sin \alpha \\
0 & \operatorname{sen} \alpha & \cos \alpha
\end{array}\right]
$$

e portanto temos a seguinte representação matricial para o operador $R_{z}(\alpha)$ :

$$
\mathrm{R}_{\mathrm{z}}(\alpha)=\mathrm{e}^{-\mathrm{i} \alpha \mathrm{L}_{\mathrm{z}}} \rightarrow\left[\begin{array}{cc}
\mathrm{R}_{\mathrm{z}}(\alpha) & 0 \\
0 & \mathrm{R}_{\mathrm{z}}(\alpha)
\end{array}\right]
$$

onde usamos a mesma notação para o operador e matriz de rotação, pois a sua distinção é óbvia no contexto. Analogamente obtemos a representação matricial de $\mathrm{R}_{\mathrm{y}}(\beta)$ :

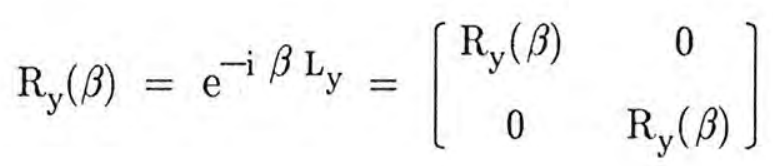

onde

$$
\mathrm{R}_{\mathrm{y}}(\beta)=\left[\begin{array}{ccc}
\cos \beta & -\operatorname{sen} \beta & 0 \\
\operatorname{sen} \beta & \cos \beta & 0 \\
0 & 0 & 1
\end{array}\right]
$$

e portanto a representação matricial do operador $\mathrm{R}(\Omega)$ é dada por:

$$
\mathrm{R}(\Omega)=\mathrm{R}_{\mathrm{z}}(\alpha) \mathrm{R}_{\mathrm{y}}(\beta) \mathrm{R}_{\mathrm{z}}(\gamma) \rightarrow\left[\begin{array}{ll}
\mathrm{R} & 0 \\
0 & \mathrm{R}
\end{array}\right]
$$


onde

$$
\mathrm{R}=\left[\begin{array}{ccc}
\cos \beta & -\operatorname{sen} \beta \cos \gamma & \operatorname{sen} \beta \sin \gamma \\
\cos \alpha \sin \beta & \cos \alpha \cos \gamma \cos \beta-\operatorname{sen} \alpha \operatorname{sen} \gamma & -\cos \alpha \operatorname{sen} \gamma \cos \beta-\operatorname{sen} \alpha \cos \gamma \\
\operatorname{sen} \alpha \operatorname{sen} \beta & \operatorname{sen} \alpha \cos \gamma \cos \beta+\cos \alpha \operatorname{sen} \gamma & -\operatorname{sen} \alpha \operatorname{sen} \gamma \cos \beta+\cos \alpha \cos \gamma
\end{array}\right]
$$

\section{A.2.2.2 - Operador Transformação de Escala}

O operador gerador de transformação de escala na direção " $\mu$ " é definido por:

$$
\mathrm{S}_{\mu}=\mathrm{i}\left[\mathrm{A}_{\mu \mu}^{+}-\mathrm{A}_{\mu \mu}\right] \quad, \quad \mu=1,2,3
$$

Usando a expresão (10), obtemos que a ação da transformação gerada pelo operador acima no vetor $\underset{\sim}{\sigma}$, definido pela eq.(3), é dada por:

$$
\begin{aligned}
\stackrel{\sim}{\sigma}^{\prime} & =\mathrm{e}^{\mathrm{W}} \underset{\sim}{\sigma} \mathrm{e}^{-\mathrm{W}}=\mathrm{e}^{\sum_{\mathrm{k}} \lambda_{\mathrm{k}}\left(\mathrm{A}_{\mathrm{kk}}^{+}-\mathrm{A}_{\mathrm{kk}}\right)} \underset{\sim}{\sigma} \mathrm{e}^{-\sum_{\mathrm{k}} \lambda_{\mathrm{k}}\left(\mathrm{A}_{\mathrm{kk}}^{+}-\mathrm{A}_{\mathrm{kk}}\right)}= \\
& =\sum_{1}\left(\cosh \lambda_{1} \mathrm{y}_{1}-\operatorname{senh} \lambda_{1} \mathrm{z}_{1}\right) \mathrm{a}_{1}^{+}+\left(-\operatorname{senh} \lambda_{1} \mathrm{y}_{1}+\cosh \lambda_{1} \mathrm{z}_{1}\right) \mathrm{a}_{1}
\end{aligned}
$$

e portanto a representação matricial é a seguinte:

$$
\mathrm{e}^{-\sum_{\mathrm{k}} \lambda_{\mathrm{k}} \mathrm{S}_{\mathrm{k}}} \rightarrow\left[\begin{array}{rr}
\cosh \underset{\sim}{\lambda} & -\underset{\sim}{\operatorname{senh}} \underset{\sim}{\lambda} \\
-\operatorname{senh} \underset{\sim}{\cosh }
\end{array}\right]
$$


onde

$$
\begin{aligned}
& \underset{\sim}{\cosh \underset{\sim}{\lambda}}=\left[\begin{array}{ccc}
\cosh \lambda_{1} & & 0 \\
& \cosh \lambda_{2} & \\
0 & & \cosh \lambda_{3}
\end{array}\right] \\
& \underset{\sim}{\operatorname{senh}} \underset{\sim}{\lambda}=\left[\begin{array}{ccc}
\operatorname{senh} \lambda_{1} & & 0 \\
& \operatorname{senh} \lambda_{2} & \\
0 & & \operatorname{senh} \lambda_{3}
\end{array}\right] .
\end{aligned}
$$

A partir da representação (31) do operador transformação de escala e das relações (23) é trivial obtermos a fórmula BCH que usamos no capítulo 4.

\section{A.2.2.3 -Representação do Operador $u(\rho)$}

O cálculo do potencial, como mostramos na seção A.1.3.1 do apêndice 1, envolve o operador

$$
\mathrm{u}(\rho)=\mathrm{e}^{-2 \rho^{2} / \mu^{2}}
$$


onde podemos escrever

$$
\rho^{2}=\sum_{\mu=1}^{3} \rho_{\mu}^{2}=\mathrm{b}_{0}^{2}\left[\mathrm{~A}_{11}+\mathrm{A}_{22}+\mathrm{A}_{33}+\mathrm{C}_{11}+\mathrm{C}_{22}+\mathrm{C}_{33}+\mathrm{A}_{11}^{+}+\mathrm{A}_{22}^{+}+\mathrm{A}_{33}^{+}\right]
$$

Os operadores acima são obtidos a partir da definição de $a_{\mu}^{+}$e $a_{\mu}$ dada no apêndice 1 , mas como já afirmamos isto é irrelevante para o cálculo da forma normal que iremos fazer. Definindo $\xi=-2 \mathrm{~b}_{0}^{2} / \mu^{2}$ obtemos

$$
\mathrm{e}^{-2 \rho^{2} / \mu^{2}}=\mathrm{e}^{\xi \sum_{\mathrm{i}=1}^{3}\left(\mathrm{~A}_{\mathrm{ii}}^{+}+\mathrm{C}_{\mathrm{ii}}+\mathrm{A}_{\mathrm{ii}}\right)}
$$

usando a eq.(10) obtemos:

$$
\begin{aligned}
\underset{\sim}{\sigma^{\prime}} & =\mathrm{e}^{\mathrm{W}} \underset{\sim}{\sigma} \mathrm{e}^{-\mathrm{W}}=\mathrm{e}^{-2 \rho^{2} / \mu^{2}} \underset{\sim}{\sigma} \mathrm{e}^{2 \rho^{2} / \mu^{2}}= \\
& =\sum_{1}\left((1+\xi) \mathrm{y}_{1}-\xi \mathrm{z}_{1}\right) \mathrm{a}_{1}^{+}+\left(\xi \mathrm{y}_{1}+(1-\xi) \mathrm{z}_{1}\right) \mathrm{a}_{1}
\end{aligned}
$$

Portanto a representação matricial de $\mathrm{u}(\rho)$ é dada por:

$$
\mathrm{u}(\rho)=\mathrm{e}^{-2 \rho^{2} / \mu^{2}} \rightarrow\left[\begin{array}{cc}
(1+\xi) \mathrm{I} & -\xi \mathrm{I} \\
\xi \mathrm{I} & (1-\xi)
\end{array}\right]
$$




\section{A.2.3 - Cálculo da Forma Normal dos Operadores de Interesse}

A seguir utilizaremos os resultados da última seção para construirmos a representação matricial dos operadores de interesse. Através das eq.(23) obtemos a forma normal de tais operadores.

\section{A.2.3.1 - Forma Normal do Operador $\hat{\mathrm{O}}_{1}$ :}

$$
\hat{\mathrm{O}}_{1}=\mathrm{e}^{\tilde{\beta}_{\perp}^{\prime} \mathrm{A}_{\perp}} \mathrm{e}^{-2 \rho^{2} / \mu^{2}} \mathrm{R}_{\mathrm{y}}(\beta) \mathrm{e}^{\tilde{\beta}_{\perp} \mathrm{A}_{\perp}^{+}}
$$

onde $\mathrm{A}_{\perp}^{+}=\mathrm{A}_{22}^{+}+\mathrm{A}_{33}^{+}$.

O operador acima é usado no cálculo da representação $0 \mathrm{p}-0 \mathrm{~b}$, como mostramos no apêndice 1. Ele aparece no cálculo do potencial e do overlap, onde, para calcularmos o overlap tomamos o limite $\mu \rightarrow \infty$.

A representação matricial do operador acima, é facilmente obtida a partir das expressões (14),(32),(28) e (12), isto é:

$$
\begin{aligned}
\hat{O}_{1} \rightarrow\left[\begin{array}{cc}
\mathrm{I} & 0 \\
\tilde{\mathrm{B}}_{\perp}^{\prime} & \mathrm{I}
\end{array}\right]\left[\begin{array}{cc}
(1+\xi) \mathrm{I} & -\xi \mathrm{I} \\
\xi \mathrm{I} & (1-\xi) \mathrm{I}
\end{array}\right]\left[\begin{array}{cc}
\mathrm{R}_{\mathrm{y}}(\beta) & 0 \\
0 & \mathrm{R}_{\mathrm{y}}(\beta)
\end{array}\right]\left[\begin{array}{ll}
\mathrm{I} & \tilde{\mathrm{B}}_{\perp} \\
0 & \mathrm{I}
\end{array}\right] \\
=\left[\begin{array}{cc}
(1+\xi) \mathrm{R}_{\mathrm{y}}(\beta) & \mathrm{R}_{\mathrm{y}}(\beta)\left((1+\xi) \tilde{\mathrm{B}}_{\perp}-\xi\right) \\
\left((1+\xi) \tilde{\mathrm{B}}_{\perp}^{\prime}+\xi\right) \mathrm{R}_{\mathrm{y}}(\beta) & \tilde{\mathrm{B}}_{\perp}^{\prime} \mathrm{R}_{\mathrm{y}}(\beta)\left((1+\xi) \tilde{\mathrm{B}}_{\perp}-\xi\right)+ \\
& \\
& +\mathrm{R}_{\mathrm{y}}(\beta)\left(\xi \tilde{\mathrm{B}}_{\perp}+(1-\xi)\right.
\end{array}\right]
\end{aligned}
$$


onde definimos:

$$
\tilde{\mathrm{B}}_{\perp}^{\prime}=\left[\begin{array}{ccc}
0 & & \\
& \tilde{\beta}_{\perp}^{\prime} & \\
& & \tilde{\beta}_{\perp}^{\prime}
\end{array}\right] \quad, \quad \tilde{\mathrm{B}}_{\perp}=\left[\begin{array}{ccc}
0 & & \\
& -\tilde{\beta}_{\perp} & \\
& & -\tilde{\beta}_{\perp}
\end{array}\right] .
$$

Comparando a eq.(33) com a eq.(5) e definindo as seguintes constantes:

$$
\begin{aligned}
& \mathrm{a}_{1}=\frac{\bar{\gamma}-1}{\bar{\gamma}} \quad \text { onde } \quad \bar{\gamma}=1+\frac{2 \mathrm{~b}_{0}^{2}}{\mu^{2}} \\
& \mathrm{~b}_{1}=\mathrm{a}_{1}^{2}-\mathrm{x}^{2} / \bar{\gamma}^{2} \\
& \mathrm{c}_{1}=\mathrm{a}_{1}^{2}-1 / \bar{\gamma}^{2} \\
& \mathrm{~W}=1+\mathrm{a}_{\perp}\left(\tilde{\beta}_{\perp}+\tilde{\beta}_{\perp}^{\prime}\right)+\mathrm{c}_{1} \tilde{\beta}_{\perp} \tilde{\beta}_{\perp}^{\prime} \\
& \mathrm{Q}=1+\mathrm{a}_{\perp}\left(\tilde{\beta}_{\perp}+\tilde{\beta}_{\perp}^{\prime}\right)+\mathrm{b}_{1} \tilde{\beta}_{\perp} \tilde{\beta}_{\perp}^{\prime}
\end{aligned}
$$

obtemos:

$$
\mathrm{B}=\mathrm{R}_{\mathrm{y}}(\beta)\left((1+\xi) \tilde{\mathrm{B}}_{\perp}-\xi\right)=\bar{\gamma} \mathrm{R}_{\mathrm{y}}(\beta)\left[\begin{array}{ccc}
\mathrm{a}_{1} & & 0 \\
& \mathrm{a}_{1}+\mathrm{c}_{1} \tilde{\beta}_{\perp} & \\
0 & & \mathrm{a}_{1}+\mathrm{c}_{1} \tilde{\beta}_{\perp}
\end{array}\right]
$$




$$
\begin{aligned}
& \mathrm{C}=\left((1+\xi) \tilde{\mathrm{B}}_{\perp}^{\prime}+\xi\right) \mathrm{R}_{\mathrm{y}}(\beta)=(-) \bar{\gamma}\left[\begin{array}{lll}
\mathrm{a}_{1} & & 0 \\
& \mathrm{a}_{1}+\mathrm{c}_{1} \tilde{\beta}_{\perp}^{\prime} & \\
0 & & \mathrm{a}_{1}+\mathrm{c}_{1} \tilde{\beta}_{\perp}^{\prime}
\end{array}\right] \mathrm{R}_{\mathrm{y}}(\beta) \\
& \mathrm{D}=\tilde{\mathrm{B}}_{\perp}^{\prime} \mathrm{B}+\mathrm{R}_{\mathrm{y}}(\beta)\left(\xi \tilde{\mathrm{B}}_{\perp}+(1-\xi)\right)=\bar{\gamma}\left[\begin{array}{ccc}
\cos \beta & -\left(1+\mathrm{a}_{1} \tilde{\beta}_{\perp}\right) \sin \beta & 0 \\
\left(1+\mathrm{a}_{1} \tilde{\beta}_{\perp}^{\prime}\right) \operatorname{sen} \beta & \mathrm{W} \cos \beta & 0 \\
0 & 0 & \mathrm{~W}
\end{array}\right] .
\end{aligned}
$$

Para utilizarmos as expressões (23) precisamos calcular a inversa da matriz D, que é dada por:

$$
\mathrm{D}^{-1}=\frac{1}{\bar{\gamma}}\left(\begin{array}{ccc}
\frac{\mathrm{W}}{\mathrm{Q}} \cos \beta & \frac{\left(1+\mathrm{a}_{1} \tilde{\beta}_{\perp}\right)}{\mathrm{Q}} \sin \beta & 0 \\
\frac{-\left(1+\mathrm{a}_{1} \tilde{\beta}_{\perp}^{\prime}\right)}{\mathrm{Q}} \sin \beta & \frac{\cos \beta}{\mathrm{Q}} & 0 \\
0 & 0 & \frac{1}{\mathrm{~W}}
\end{array}\right]
$$


e portanto a partir das expressões (23) obtemos:

$$
\mathrm{h}=-\left(\mathrm{B} \mathrm{D}^{-1}\right)=\left[\begin{array}{ccc}
\frac{-\mathrm{a}_{1} \mathrm{~W}+\frac{\operatorname{sen}^{2} \beta}{\bar{\gamma}^{2}} \tilde{\beta}_{\perp}}{\mathrm{Q}} & \frac{-\operatorname{sen} \beta \cos \beta \tilde{\beta}_{\perp}}{\bar{\gamma}^{2} \mathrm{Q}} & 0 \\
\frac{-\operatorname{sen} \beta \cos \beta \tilde{\beta}_{\perp}}{\bar{\gamma}^{2} \mathrm{Q}} & \frac{-\left(\mathrm{a}_{1}+\mathrm{b}_{1} \tilde{\beta}_{\perp}\right)}{\mathrm{Q}} & 0 \\
0 & 0 & \frac{-\left(\mathrm{a}_{1}+\mathrm{c}_{1} \tilde{\beta}_{\perp}\right)}{\mathrm{W}}
\end{array}\right]
$$

$$
\mathrm{h}^{\prime}=\mathrm{D}^{-1} \mathrm{C}=\left[\begin{array}{ccc}
\frac{-\mathrm{a}_{1} \mathrm{~W}+\frac{\operatorname{sen}^{2} \beta}{\bar{\gamma}^{2}} \tilde{\beta}_{\perp}^{\prime}}{\mathrm{Q}} & \frac{\operatorname{sen} \beta \cos \beta \tilde{\beta}_{\perp}^{\prime}}{\bar{\gamma}^{2} \mathrm{Q}} & 0 \\
\frac{\operatorname{sen} \beta \cos \beta \tilde{\beta}_{\perp}^{\prime}}{\bar{\gamma}^{2} \mathrm{Q}} & \frac{-\left(\mathrm{a}_{1}+\mathrm{b}_{1} \tilde{\beta}_{\perp}^{\prime}\right)}{\mathrm{Q}} & 0 \\
0 & 0 & \frac{-\left(\mathrm{a}_{1}+\mathrm{c}_{1} \tilde{\beta}_{\perp}^{\prime}\right)}{\mathrm{W}}
\end{array}\right]
$$

e como $g=\left(D^{t}\right)^{-1}$, a partir das eq.(19) obtemos: 


$$
\begin{aligned}
& \bar{\alpha}_{11}=\ln \left[\frac{1}{\bar{\gamma}} \frac{\mathrm{W}}{\mathrm{Q}} \cos \beta\right] \quad \bar{\alpha}_{22}=\ln \left[\frac{1}{\bar{\gamma} \mathrm{W} \cos \beta}\right] \quad \bar{\alpha}_{33}=\ln \left[\frac{1}{\bar{\gamma} \mathrm{W}}\right] \\
& \bar{\alpha}_{12}=\frac{-\left(1+\mathrm{a}_{1} \tilde{\beta}_{\perp}^{\prime}\right)}{\mathrm{W}} \frac{\sin \beta}{\cos \beta} \quad \bar{\alpha}_{13}=0 \quad \bar{\alpha}_{23}=0 \\
& \bar{\alpha}_{21}=\frac{\left(1+\mathrm{a}_{1} \tilde{\beta}_{\perp}\right)}{\mathrm{W}} \frac{\sin \beta}{\cos \beta} \quad \bar{\alpha}_{31}=0 \quad \bar{\alpha}_{32}=0 .
\end{aligned}
$$

As expressões (34),(35) e (36) geram a forma normal dada pela eq.(23) que procuramos.

\section{A.2.3.2 - Forma Normal do Operador $\hat{\mathrm{O}}_{2}$ :}

$$
\hat{\mathrm{O}}_{2}=\mathrm{e}^{\tilde{\beta}_{\|}^{\prime} \mathrm{A}_{\|}} \mathrm{e}^{-2 \rho^{2} / \mu^{2}} \mathrm{R}(\Omega) \mathrm{e}^{\tilde{\beta}_{\| 1} \mathrm{~A}_{\|}^{+}} .
$$

$\mathrm{O}$ operador acima é envolvido no cálculo das representações $2 \mathrm{p}-2 \mathrm{~b}$ e $4 \mathrm{p}-4 \mathrm{~b}$ quando calculamos o potencial e o overlap $(\mu \rightarrow \infty)$, como mostramos no apêndice 1 .

A partir das expressões (14),(32),(29) e (12) obtemos a representação matricial de $\hat{\mathrm{O}}_{2}$, seguinte: 


$$
\begin{aligned}
& \hat{\mathrm{O}}_{2} \rightarrow\left[\begin{array}{cc}
\mathrm{I} & 0 \\
\tilde{\mathrm{B}}_{\prime \prime}^{\prime \prime} & \mathrm{I}
\end{array}\right]\left[\begin{array}{cc}
(1+\xi) \mathrm{I} & -\xi \mathrm{I} \\
\xi \mathrm{I} & (1-\xi) \mathrm{I}
\end{array}\right]\left[\begin{array}{cc}
\mathrm{R} & 0 \\
0 & \mathrm{R}
\end{array}\right]\left[\begin{array}{cc}
\mathrm{I} & \tilde{\mathrm{B}}_{\prime \prime} \\
0 & \mathrm{I}
\end{array}\right]
\end{aligned}
$$

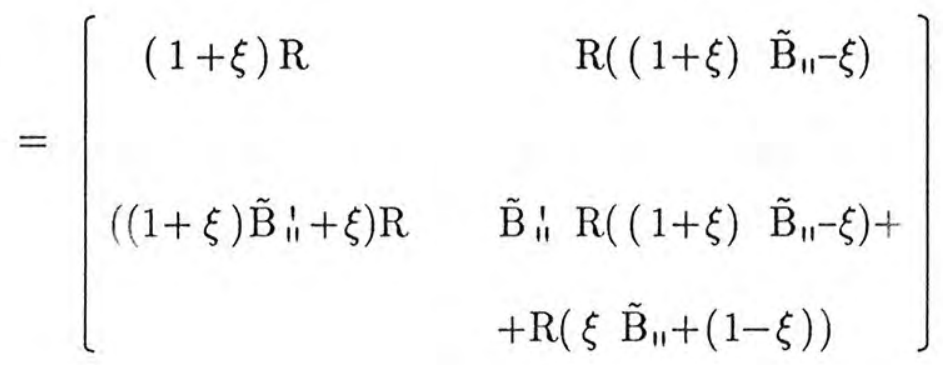

onde definimos:

$$
\tilde{\mathrm{B}}_{\|}^{\prime}=\left[\begin{array}{ccc}
\tilde{\beta}_{\| 1}^{\prime} & & 0 \\
& 0 & \\
0 & & 0
\end{array}\right] \quad, \quad \tilde{\mathrm{B}}_{\| 1}=\left[\begin{array}{ccc}
-\tilde{\beta}_{11} & & 0 \\
& 0 & \\
0 & & 0
\end{array}\right] \text {. }
$$

Comparando a eq.(37) com (5) obtemos:

$$
\begin{aligned}
& B=\bar{\gamma} R\left[\begin{array}{lll}
a_{1}+c_{1} \tilde{\beta}_{\prime \prime} & & 0 \\
& a_{1} & \\
0 & & a_{1}
\end{array}\right] \\
& C=-\bar{\gamma}\left[\begin{array}{lll}
a_{1}+c_{1} \tilde{\beta}_{\prime \prime}^{\prime \prime} & & 0 \\
0 & a_{1} & \\
0 & & a_{1}
\end{array}\right] R
\end{aligned}
$$


$\mathrm{D}=\bar{\gamma} \cdot\left[\begin{array}{ccc}\overline{\mathrm{W}} \cos \beta & -\left(1+\mathrm{a}_{1} \tilde{\beta}_{11}^{\prime}\right) \cos \gamma \sin \beta & \left(1+\mathrm{a}_{1} \tilde{\beta}_{1 \prime}^{\prime}\right) \operatorname{sen} \gamma \sin \beta \\ \left(1+\mathrm{a}_{1} \tilde{\beta}_{11}\right) \cos \alpha \sin \beta & \cos \alpha \cos \gamma \cos \beta-\operatorname{sen} \alpha \operatorname{sen} \gamma & -(\cos \alpha \cos \beta \operatorname{sen} \gamma+\operatorname{sen} \alpha \cos \gamma) \\ \left(1+\mathrm{a}_{1} \tilde{\beta}_{11}\right) \operatorname{sen} \alpha \sin \beta & \operatorname{sen} \alpha \cos \gamma \cos \beta+\cos \alpha \sin \gamma & -\operatorname{sen} \alpha \operatorname{sen} \gamma \cos \beta+\cos \alpha \cos \gamma\end{array}\right]$

e pelo cálculo direto

$\mathrm{D}^{\prime 1}=\frac{1}{\bar{\gamma}} \cdot\left[\begin{array}{ccc}\frac{\cos \beta}{\bar{Q}} & \frac{\left(1+\mathrm{a}_{1} \tilde{\beta}_{11}^{\prime}\right)}{\bar{Q}} \operatorname{sen} \beta \cos \alpha & \frac{\left(1+\mathrm{a}_{1} \tilde{\beta}_{11}^{\prime}\right)}{\bar{Q}} \operatorname{sen} \operatorname{sen} \beta \\ \frac{-\left(1+\mathrm{a}_{1} \tilde{\beta}_{11}\right)}{\bar{Q}} \operatorname{sen} \beta \cos \gamma & \frac{\cos \alpha \cos \gamma \cos \beta \overline{\mathrm{W}}-\operatorname{sen} \alpha \operatorname{sen} \gamma \overline{\mathrm{Q}}}{\overline{\mathrm{Q}}} & \frac{\operatorname{sen} \alpha \cos \gamma \cos \beta \overline{\mathrm{W}}+\cos \alpha \operatorname{sen} \gamma \overline{\mathrm{Q}}}{\overline{\mathrm{Q}}} \\ \frac{\left(1+\mathrm{a}_{1} \tilde{\beta}_{11}\right)}{\overline{\mathrm{Q}}} \operatorname{sen} \beta \sin \gamma & \frac{-\cos \alpha \sin \gamma \cos \beta \overline{\mathrm{W}}-\operatorname{sen} \alpha \cos \gamma \overline{\mathrm{Q}}}{\overline{\mathrm{Q}}} & \frac{-\operatorname{sen} \alpha \operatorname{sen} \gamma \cos \beta \overline{\mathrm{W}}+\cos \alpha \cos \gamma \overline{\mathrm{Q}}}{\overline{\mathrm{Q}}}\end{array}\right]$

onde definimos

$$
\begin{aligned}
& \overline{\mathrm{W}}=1+\mathrm{a}_{1}\left(\tilde{\beta}_{11}^{\prime}+\tilde{\beta}_{11}\right)+\mathrm{c}_{1} \tilde{\beta}_{11} \tilde{\beta}_{11}^{\prime} \\
& \overline{\mathrm{Q}}=1+\mathrm{a}_{1}\left(\tilde{\beta}_{11}^{\prime}+\tilde{\beta}_{11}\right)+\mathrm{b}_{1} \tilde{\beta}_{11} \tilde{\beta}_{11}^{\prime}
\end{aligned}
$$

e portanto a partir das expressões (23) obtemos: 


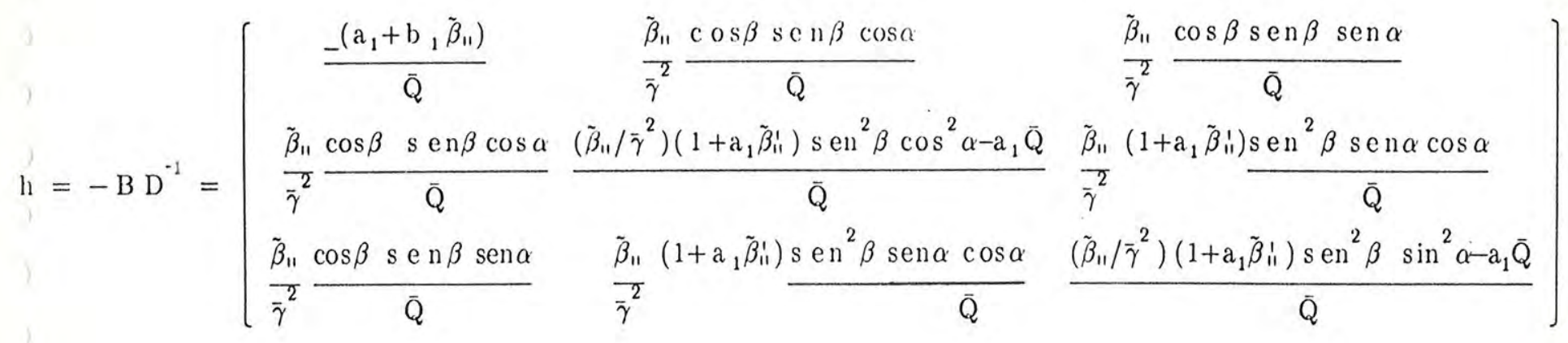

(38)

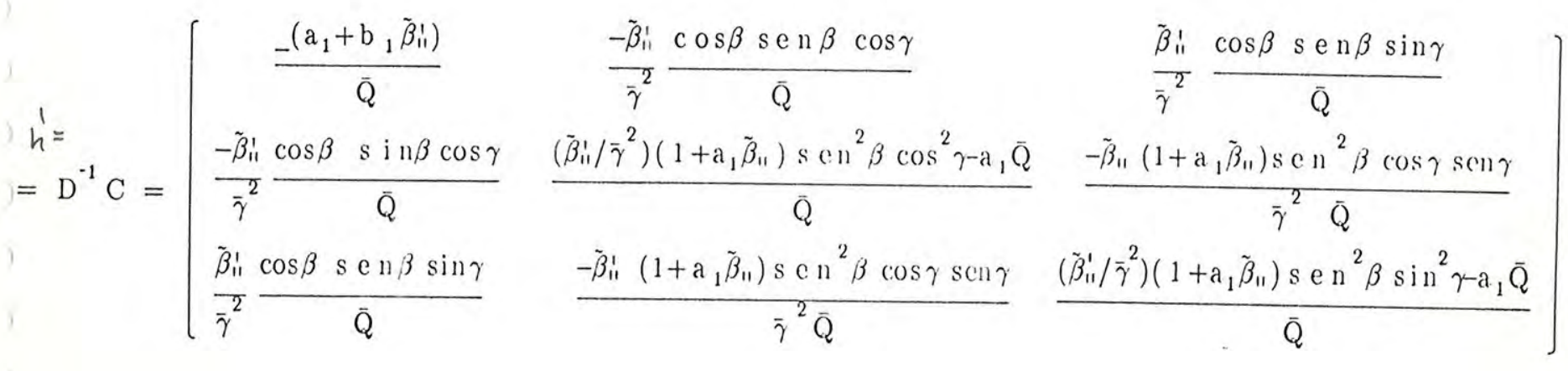


e finalmente como $g=\left(D^{t}\right)^{-1}$, de (19) obtemos:

$$
\begin{aligned}
& \bar{\alpha}_{11}=\ln \left[\frac{1}{\bar{\gamma}} \frac{\cos \beta}{\bar{Q}}\right] \quad \bar{\alpha}_{22}=\ln \left[\frac{1}{\bar{\gamma} \cos \beta}\right] \quad \bar{\alpha}_{33}=\ln \left[\frac{1}{\bar{\gamma}} \frac{1}{\bar{\Omega}}\right] \\
& \bar{\alpha}_{12}=-\left(1+a_{1} \tilde{\beta}_{11}\right) \operatorname{tag} \beta \cos \gamma \quad \bar{\alpha}_{13}=\left(1+a_{1} \tilde{\beta}_{11}\right) \operatorname{tag} \beta \operatorname{sen} \gamma \\
& \bar{\alpha}_{23}=\frac{-(\cos \alpha \operatorname{sen} \gamma+\sin \alpha \cos \gamma \cos \beta)}{\bar{\Omega}} \\
& \bar{\alpha}_{21}=\left(1+a_{1} \tilde{\beta}_{1 \prime}^{\prime}\right) \operatorname{tag} \beta \cos \alpha \\
& \bar{\alpha}_{32}=\frac{(\operatorname{sen} \alpha \cos \gamma+\cos \alpha \operatorname{sen} \gamma \cos \beta)}{\bar{\Omega}}
\end{aligned}
$$

onde definimos:

$$
\bar{\Omega}=\cos \alpha \cos \gamma-\operatorname{sen} \alpha \operatorname{sen} \gamma \cos \beta .
$$

As equações (38),(39) e (40) e as relações (23) resultam na forma normal procurada para o operador $\hat{\mathrm{O}}_{2}$. 


\section{Apêndice 3 - Cálculo dos Coeficientes de Talmi-Moshinsky}

Neste apêndice vamos obter os coeficientes ${ }^{(26)}$ que determinam a transformação da função de onda do par de nucleons, para suas componentes relativas e do centro de massa, que utilizamos no apêndice 1 para obter os elementos de matriz do potencial.

Usando a mesma notação do capítulo 4, temos que

$$
\left|\mathrm{p}_{1} \mathrm{q}_{1} \mathrm{r}_{1}, \mathrm{p}_{2} \mathrm{q}_{2} \mathrm{r}_{2}>=\sum_{\substack{\mathrm{pqr} \\ \mathrm{PQR}}}\right| \text { pqr, PQR }><\text { pqr, PQR } \mid \mathrm{p}_{1} \mathrm{q}_{1} \mathrm{r}_{1}, \mathrm{p}_{2} \mathrm{q}_{2} \mathrm{r}_{2}>
$$

onde $\mathrm{p}_{1} \mathrm{q}_{1} \mathrm{r}_{1}, \mathrm{p}_{2} \mathrm{q}_{2} \mathrm{r}_{2}$ correspondem aos números quânticos do oscilador, na base cartesiana, associados aos estados ocupados pelos nucleons 1 e 2 e pqr, PQR correspondem aos números quânticos do oscilador associado ao movimento relativo e do centro de massa respectivamente.

Os coeficientes de Talmi-Moshinsky, CTM, para funções de onda do oscilador na base cartesiana, possuem a seguinte fatoração:

$$
<\text { pqr, PQR }\left|\mathrm{p}_{1} \mathrm{q}_{1} \mathrm{r}_{1}, \mathrm{p}_{2} \mathrm{q}_{2} \mathrm{r}_{2}>=<\mathrm{pP}\right| \mathrm{p}_{1} \mathrm{p}_{2}><\mathrm{qQ}\left|\mathrm{q}_{1} \mathrm{q}_{2}><\mathrm{rR}\right| \mathrm{r}_{1} \mathrm{r}_{2}>
$$

onde os fatores a direita se referem a transformações nas direções 1, 2 e 3, separadamente. Portanto, basta calcularmos os fatores $<\mathrm{pP} \mid \mathrm{p}_{1} \mathrm{p}_{2}>$ e utilizarmos a expressão (2) para obtermos os CTM.

O operador criação de quanta de oscilador, em relação a um referencial fixo no espaço, é definido por: 


$$
\mathrm{a}_{\mu ; \mathrm{i}}^{+}=\frac{1}{\sqrt{2}}\left[\frac{\mathrm{x}_{\mu}(\mathrm{i})}{\mathrm{b}_{0}}-\mathrm{i} \frac{\mathrm{b}_{0}}{\hbar} \mathrm{p}_{\mu}(\mathrm{i})\right]
$$

e portanto

$$
\left|\mathrm{p}_{1} \mathrm{p}_{2}\right\rangle=\frac{1}{\left(\mathrm{p}_{1} ! \mathrm{p}_{2} !\right)^{1 / 2}}\left(\mathrm{a}_{1 ; 1}^{+}\right)^{\mathrm{p}_{1}}\left(\mathrm{a}_{1 ; 2}^{+}\right)^{\mathrm{p}_{2}} \mid 0>
$$

Como mostramos no apêndice 1, obtemos as coordenadas canônicas relativas e de centro de massa do par de nucleons através da seguinte transformação de coordenadas:

$$
\begin{array}{ll}
\rho_{\mu}=\frac{1}{\sqrt{2}} \mathrm{x}_{\mu}(1)-\frac{1}{\sqrt{2}} \mathrm{x}_{\mu}(2) & \pi_{\mu}=\frac{1}{\sqrt{2}} \mathrm{p}_{\mu}(1)-\frac{1}{\sqrt{2}} \mathrm{p}_{\mu}(2) \\
\mathrm{R}_{\mu}=\frac{1}{\sqrt{2}} \mathrm{x}_{\mu}(1)+\frac{1}{\sqrt{2}} \mathrm{x}_{\mu}(2) & \mathrm{P}_{\mu}=\frac{1}{\sqrt{2}} \mathrm{p}_{\mu}(1)+\frac{1}{\sqrt{2}} \mathrm{p}_{\mu}(2)
\end{array}
$$

e portanto definindo os operadores de criação de quanta de oscilador associado ao movimento relativo e do centro e de centro de massa por:

$$
\begin{aligned}
& \mathrm{a}_{\mu}^{+}=\frac{1}{\sqrt{2}}\left[\frac{\rho_{\mu}}{\mathrm{b}_{0}}-\mathrm{i} \frac{\mathrm{b}_{0}}{\hbar} \pi_{\mu}\right] \\
& \mathrm{a}_{\mu}^{+\mathrm{CM}}=\frac{1}{\sqrt{2}}\left[\frac{\mathrm{R}_{\mu}}{\mathrm{b}_{0}}-\mathrm{i} \frac{\mathrm{b}_{0}}{\hbar} \mathrm{P}_{\mu}\right]
\end{aligned}
$$


obtemos

$$
\begin{aligned}
& \mathrm{a}_{\mu}^{+}=\frac{1}{\sqrt{2}}\left[\mathrm{a}_{\mu ; 1}^{+}-\mathrm{a}_{\mu ; 2}^{+}\right] \\
& \mathrm{a}_{\mu}^{+} \mathrm{CM}=\frac{1}{\sqrt{2}}\left[\mathrm{a}_{\mu ; 1}^{+}+\mathrm{a}_{\mu ; 2}^{+}\right]
\end{aligned}
$$

e portanto

$$
\begin{aligned}
& \mathrm{a}_{\mu ; 1}^{+}=\frac{1}{\sqrt{2}}\left[\mathrm{a}_{\mu}^{+} \mathrm{CM}+\mathrm{a}_{\mu}^{+}\right] \\
& \mathrm{a}_{\mu ; 2}^{+}=\frac{1}{\sqrt{2}}\left[\mathrm{a}_{\mu}^{+} \mathrm{CM}-\mathrm{a}_{\mu}^{+}\right] .
\end{aligned}
$$

Substituindo as expressões acima em (3) obtemos:

$$
\begin{aligned}
& \left|p_{1} p_{2}\right\rangle=\frac{1}{\left(p_{1} ! p_{2} ! 2^{p_{1}+p_{2}}\right)^{1 / 2}}\left[a_{1}^{+} C M+a_{1}^{+}\right]^{p_{1}}\left[a_{1}^{+} C M-a_{1}^{+}\right]^{p_{2}} \mid 0>= \\
& =\frac{1}{\left(p_{1} ! p_{2} ! 2^{p_{1}+p_{2}}\right)^{1 / 2}} \sum_{m_{1}=0}^{p_{1}}\left(\begin{array}{l}
p_{1} \\
m_{1}
\end{array}\right)\left[a_{1}^{+} C M\right]^{p_{1}-m_{1}}\left[a_{1}^{+}\right]^{m_{1}} \sum_{m_{2}=0}^{p_{2}}\left[\begin{array}{l}
p_{2} \\
m_{2}
\end{array}\right]\left[a_{1}^{+} C M\right]^{p_{2}-m_{2}}\left[-a_{1}^{+}\right]^{m_{2}} \mid 0> \\
& =\left[\frac{p_{1} ! p_{2} !}{2^{p_{1}+p_{2}}}\right]^{1 / 2} \sum_{m_{1}=0}^{p_{1}} \sum_{m_{2}=0}^{p_{2}} \frac{(-1)^{m_{2}}}{\left(p_{1}-m_{1}\right) ! m_{1} !\left(p_{2}-m_{2}\right) ! m_{2} !}\left[a_{1}^{+} C M\right]^{p_{1}+p_{2}-\left(m_{1}+m_{2}\right)}\left[a_{1}^{+}\right]^{m_{1}+m_{2}} \mid 0>
\end{aligned}
$$


fazendo $\mathrm{m}_{1}+\mathrm{m}_{2}=\mathrm{p}$, podemos escrever

$$
\begin{aligned}
& \mid \mathrm{p}_{1} \mathrm{p}_{2}>=\sum_{\mathrm{p}}\left[\frac{\mathrm{p}_{1} ! \mathrm{p}_{2} !\left(\mathrm{p}_{1}+\mathrm{p}_{2}-\mathrm{p}\right) ! \mathrm{p} !}{2^{\mathrm{p}_{1}{ }^{+} \mathrm{p}_{2}}}\right]^{1 / 2} \sum_{\mathrm{m}_{2}}\left[\frac{(-1)^{\mathrm{m}_{2}}}{\left(\mathrm{p}_{1}-\mathrm{p}+\mathrm{m}_{2}\right) !\left(\mathrm{p}-\mathrm{m}_{2}\right) !\left(\mathrm{p}_{2}-\mathrm{m}_{2}\right) ! \mathrm{m}_{2} !}\right] . \\
& \cdot \frac{\left(\mathrm{a}_{1}^{+} \mathrm{CM}\right)^{\mathrm{p}_{1}+\mathrm{p}_{2}-\mathrm{p}}}{\left[\left(\mathrm{p}_{1}+\mathrm{p}_{2}-\mathrm{p}\right) !\right]^{1 / 2}} \frac{\left(\mathrm{a}_{1}^{+}\right)^{\mathrm{p}}}{(\mathrm{p} !)^{1 / 2}} \mid 0>.
\end{aligned}
$$

Agora como

$$
\left|\mathrm{pP}>=\frac{\left(\mathrm{a}_{1}^{+}\right)^{\mathrm{p}}}{\sqrt{\mathrm{p} !}} \frac{\left(\mathrm{a}_{1}^{+} \mathrm{CM}\right)^{\mathrm{P}}}{\sqrt{\mathrm{P} !}}\right| 0>
$$

Comparando com (5) obtemos

$$
<\mathrm{pP} \mid \mathrm{p}_{1} \mathrm{p}_{2}>=\left[\frac{\mathrm{p}_{1} ! \mathrm{p}_{2} ! \mathrm{p} ! \mathrm{P} !}{2^{\mathrm{p}+\mathrm{P}}}\right]^{1 / 2} \delta\left(\mathrm{p}_{1}+\mathrm{p}_{2}-\mathrm{p}-\mathrm{P}\right) \sum_{\alpha} \frac{(-1)^{\alpha}}{\left(\mathrm{p}_{1}-\mathrm{p}+\alpha\right) !\left(\mathrm{p}_{2}-\alpha\right) !(\mathrm{p}-\alpha) ! \alpha !}
$$

onde $\delta\left(\mathrm{p}_{1}+\mathrm{p}_{2}-\mathrm{p}-\mathrm{P}\right)$ indica que o coeficiente $<\mathrm{pP}\left|\mathrm{p}_{1} \mathrm{p}_{2}\right\rangle$ só é diferente de zero se $\mathrm{p}_{1}+\mathrm{p}_{2}=\mathrm{p}+\mathrm{P}$ e a soma é feita para todos os valores de $\alpha$ possíveis.

É fácil obter as seguintes relações de simetria:

$$
\begin{aligned}
& <\mathrm{pP}\left|\mathrm{p}_{1} \mathrm{p}_{2}>=(-1)^{\mathrm{p}}<\mathrm{pP}\right| \mathrm{p}_{2} \mathrm{p}_{1}> \\
& <\mathrm{pP}\left|\mathrm{p}_{1} \mathrm{p}_{2}>=(-1)^{\mathrm{p}_{2}}<\mathrm{Pp}\right| \mathrm{p}_{1} \mathrm{p}_{2}>.
\end{aligned}
$$


A partir de (6),(7) e (2) obtemos os CTM necessários aos cálculos do elemento de matriz do potencial. A seguir damos os resultados de tais cálculos, para os casos de interesse:

$$
\begin{aligned}
& <00 \mid 00>=1 \\
& <01|01\rangle=\langle 01 \mid 10\rangle=-<10|01\rangle=\langle 10 \mid 10\rangle=\frac{1}{\sqrt{2}} \\
& <11 \mid 11>=0 \text { (simetria) } \\
& <11|20>=-<11| 02>=\frac{1}{\sqrt{2}} \\
& <02|11>=-<20| 11>=\frac{1}{\sqrt{2}} \\
& <02|02>=<02| 20>=<20|02>=<20| 20>=\frac{1}{2} \\
& <03|12>=<03| 21>=<30|12>=(-)<30| 21>=\frac{1}{2} \sqrt{\frac{3}{2}} \\
& <12|12\rangle=-<12|21\rangle=\langle 21 \mid 21\rangle=\langle 21 \mid 12\rangle=(-) \frac{1}{2} \frac{1}{\sqrt{2}} \\
& <04|22>=<40| 22>=\frac{1}{2} \sqrt{\frac{3}{2}}
\end{aligned}
$$




$$
\begin{aligned}
& <13|22>=<31| 22>=0 \text { (simetria) } \\
& <22 \mid 22>=-\frac{1}{2} .
\end{aligned}
$$

A partir dos coeficientes acima, podemos através de (2), obter todos os CTM que vamos necessitar. Por exemplo:

$$
\begin{aligned}
& <000,000|000,000\rangle=<00|00><00| 00\rangle<00|00\rangle=1 \\
& <100,000|100,000\rangle=<10|10><00| 00\rangle<00|00\rangle=\frac{1}{\sqrt{2}} \\
& <000,100|100,000\rangle=<01|10><00| 00><00 \mid 00>=\frac{1}{\sqrt{2}}
\end{aligned}
$$

e de maneira análoga obtemos todos os CTM necessários. 


\section{Referências}

1. D.J. Rowe, Rep. Prog. Phys. 48 (1985) 1419-1480.

2. J.P. Elliott, Proc. Roy. Soc. A245 (1958) 128, 562.

3. F. Arickx et al., Nucl. Phys. A318 (1979) 269-286.

4. F. Arickx et al., Nucl. Phys. A377 (1982) 121-136.

5. G.F. Filippov et al., Sov. J. Part. Nucl. 16 (1985) 153-177.

6. G.F. Filippov et al., Sov. J. Part. Nucl. 32 (1980) 27-43.

7. V.I. Ovcharenko et al., Sov. J. Nucl. Phys. 35 (1982) 372-377.

8. I.P. Okrimenko et al., Sov. J. Nucl. Phys. 34 (1982) 488-494.

9. S.S. Avancini e E.J.V. de Passos, J. Phys. G: Nucl. Part. Phys. 15 (1989) 161-172.

10. Y. Agrall et al., Nucl. Phys. A131 (1969) 609-622.

11. M. Bouten et al., Prog. in Part. and Nucl. Phys. 5 (1981) 55-78.

12. F. Ajzenberg-Selove, Nucl. Phys. A433 (1985) 55.

13. S.S. Avancini - Dissertação de Mestrado - "O Modelo Simplético e seus Submodelos" - IFUSP (1986).

14. J.P. Elliott em "Selected Topics in Nuclear Theory", IAEA, Viena, 1963, ed. F. Janouch.

15. M. Bouten et al., Nucl. Phys. A158 (1970) 217-224.

16. J.D. Vergados, Nucl. Phys. A111 (1968) 681-754.

17. G.F. Filippov et al., Sov. J. Part. Nucl. 15 (1984) 600-619.

18. D.M. Brink - Proceedings of the International School of Physics, "Enrico Fermi", curso XXXVI, Varena, 1966, ed. C. Bloch.

19. D.M. Brink and E. Boecker, Nucl. Phys. A91 (1967) 1-26. 
20. R.M. Asherova et al., Nucl. Phys. A355 (1981) 25-44.

21. N. Takigawa e A. Arima, Nucl. Phys. A168 (1971) 593-624.

22. A.B. Volkov, Nucl. Phys. 74 (1965) 33.

23. Uegaki et ali - Prog. Th. Phys. 62 (1979) 1621-1640.

24. M. Moshinsky, Nucl. Phys. 13 (1959) 104-116.

25. D.J. Rowe, J. Math Phys. 26 (1985) 2787-2791.

26. D.M. Brink, Nucl. Phys. 40 (1963) 593. 